\title{
Investigation of High Reactivity Fuel Property Effects on Reactivity Controlled Compression Ignition Combustion
}

\author{
Ross H. Ryskamp
}

Follow this and additional works at: https://researchrepository.wvu.edu/etd

\section{Recommended Citation}

Ryskamp, Ross H., "Investigation of High Reactivity Fuel Property Effects on Reactivity Controlled Compression Ignition Combustion" (2016). Graduate Theses, Dissertations, and Problem Reports. 6548. https://researchrepository.wvu.edu/etd/6548

This Dissertation is protected by copyright and/or related rights. It has been brought to you by the The Research Repository @ WVU with permission from the rights-holder(s). You are free to use this Dissertation in any way that is permitted by the copyright and related rights legislation that applies to your use. For other uses you must obtain permission from the rights-holder(s) directly, unless additional rights are indicated by a Creative Commons license in the record and/ or on the work itself. This Dissertation has been accepted for inclusion in WVU Graduate Theses, Dissertations, and Problem Reports collection by an authorized administrator of The Research Repository @ WVU.

For more information, please contact researchrepository@mail.wvu.edu. 


\title{
Investigation of High Reactivity Fuel Property Effects on Reactivity Controlled Compression Ignition Combustion
}

\author{
Ross H. Ryskamp
}

\author{
Dissertation Submitted \\ to the Benjamin M. Statler College of Engineering and Mineral Resources \\ at West Virginia University \\ in partial fulfillment of the requirements for the degree of \\ Doctor of Philosophy in \\ Mechanical Engineering
}

\author{
Gregory Thompson, Ph.D., Chair \\ Nigel Clark, Ph.D. \\ Hailin Li, Ph.D. \\ Mridul Gautam, Ph.D. \\ John Nuszkowski, Ph.D. \\ William Cannella, Ph.D.
}

Mechanical and Aerospace Engineering

Morgantown, West Virginia

2016

Keywords: Diesel, Natural Gas, RCCl, Advanced Combustion, Exhaust Emissions, Engine Copyright 2016 Ross Ryskamp 


\section{Abstract}

\section{Investigation of High Reactivity Fuel Property Effects on Reactivity Controlled Compression Ignition Combustion}

\section{Ross H. Ryskamp}

Reactivity controlled compression ignition ( $\mathrm{RCCl}$ ) is a form of dual-fuel combustion that exploits the reactivity difference between two fuels to control combustion phasing. This combustion approach limits the formation of oxides of nitrogen $\left(\mathrm{NO}_{\mathrm{x}}\right)$ and soot while retaining high thermal efficiencies associated with compression-ignition $(\mathrm{Cl})$ engines. Theoretical and applied research has been conducted by researchers at several other institutions detailing RCCI combustion characteristics, exhaust emissions, fuel efficiency, and operability. However, the discussion of fuel property effects on $\mathrm{RCCl}$ combustion has been limited. Previous research on fuel properties that influence $\mathrm{RCCl}$ combustion have predominantly focused on the low reactivity fuel. The research presented herein was performed to determine the influences that high reactivity fuel properties have on $\mathrm{RCCl}$ combustion characteristics, exhaust emissions, fuel efficiency, and the operable load range.

A General Motors 4-cylinder, 1.9 liter, light-duty $\mathrm{Cl}$ engine was converted to run on diesel fuel (high reactivity fuel) and compressed natural gas (CNG) (low reactivity fuel). The engine was operated at 2100 revolutions per minute (RPM), which is near its intermediate speed and where previous low temperature combustion (LTC) research has been performed. Two different loads were imposed on the engine, 3.6 bar brake mean effective pressure (BMEP) and 6 bar BMEP. A preliminary parametric study was conducted at each load to determine which engine control parameters had the largest effect on $\mathrm{RCCl}$ combustion, exhaust emissions, and fuel efficiency. From this study, a test matrix was developed that varied intake manifold air pressure (IMAP) and the location of 50 percent mass fraction burned (CA50) for the 3.6 bar BMEP load condition. At the 6 bar BMEP load condition a test matrix that varied the direct injection (DI) start of injection (SOI) timing and CA50 was developed. CA50 was controlled by adjusting the ratio of CNG to diesel (percentage CNG). The engine was operated at each point of these test matrices with nine different diesel fuels that had varying fuel properties, including cetane number $(\mathrm{CN})$, aromatic content $(\mathrm{AC})$, and distillation temperatures. The results from these tests were used to identify high reactivity fuel property effects on $\mathrm{RCCl}$ combustion characteristics, exhaust emissions, fuel efficiency, and the operable load range.

Results from the experiment demonstrated that $\mathrm{CN}$ of the diesel fuel had a dominant effect on nearly all facets of RCCl combustion, exhaust emissions, and fuel efficiency. RCCl operation with diesel fuels whose $\mathrm{CN}$ was lower than 33 resulted in substantially higher $\mathrm{NO}_{\mathrm{x}}$ emissions and in-cylinder pressure rise rates (PRRs) that limited the operable load range, compared to fuels with a $\mathrm{CN}$ ranging from 44 to 54 . High CN diesel fuels with a low $\mathrm{AC}(<23 \%)$ required the largest percentage CNG to maintain combustion phasing, $70.5 \%$ to $78.6 \%$ of total fuel energy input as CNG at 3.6 bar BMEP and $73.4 \%$ to $83.0 \%$ at 6 bar BMEP. High CN, low AC diesel fuels also operated at the highest fuel conversion efficiency, $27.3 \%$ to $30.2 \%$ at 3.6 bar BMEP and $38.0 \%$ to $39.4 \%$ at 6 bar BMEP. Furthermore, in-cylinder PRR decreased as $\mathrm{CN}$ of the diesel fuel increased which would allow for higher engine loads to be achieved. 


\section{Acknowledgments}

Throughout my tenure as a graduate research assistant with the Center for Alternative Fuels, Engines, and Emissions (CAFEE) at West Virginia University (WVU), I have had the pleasure of interacting with many other students, staff, and faculty. To those I have not mentioned I am forever grateful for these interactions, as well as your support in my education and research endeavors. Dr. Gregory Thompson, thank you for not only your support and guidance as my research advisor, but also as my professor for many classes. Your contribution to my education and my development as an engineer is immeasurable. Thank you to my committee members, Dr. Nigel Clark, Dr. Hailin Li, Dr. Mridul Gautam, Dr. John Nuszkowski, and Dr. William Cannella. I am indebted to all of you for the expertise and guidance you have provided throughout my graduate studies. Daniel Carder, thank you for your never-ending support and advice throughout my tenure with CAFEE. I have learned an immense amount under your direction and without your help this research would not have been completed. Clara Rhodes, thank you for working beside me to setup this experiment, but more importantly, thank you for supporting me and reassuring me even when I doubted myself. Finally, thank you to my family. I could have never made it this far without your unconditional support and encouragement. 


\section{Table of Contents}

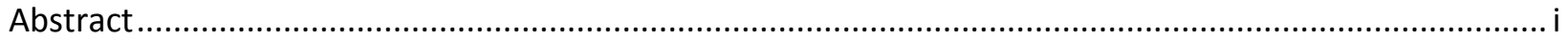

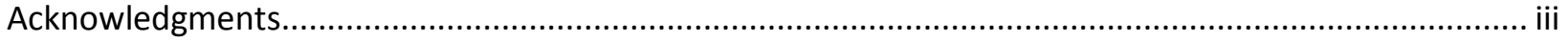

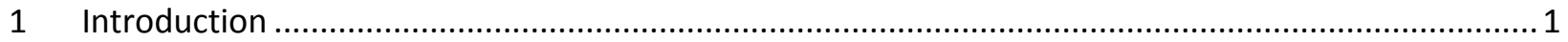

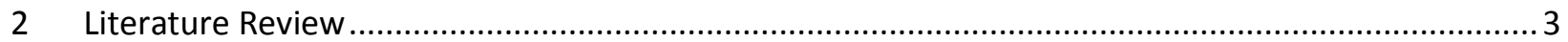

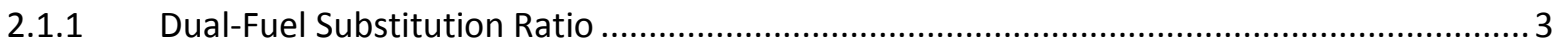

2.1.2 Emissions from Dual-Fuel Compression-Ignition Engines.................................................. 3

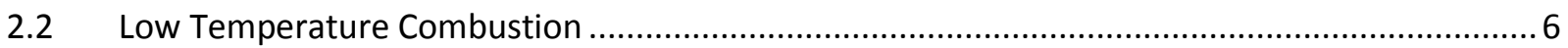

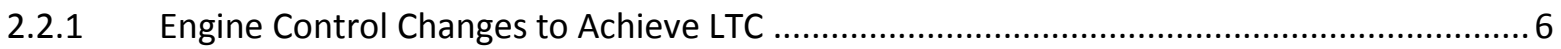

2.3 Reactivity Controlled Compression-Ignition ......................................................................... 8

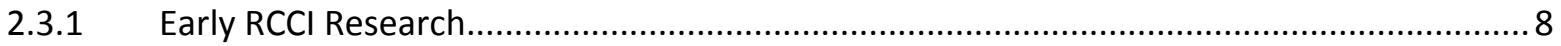

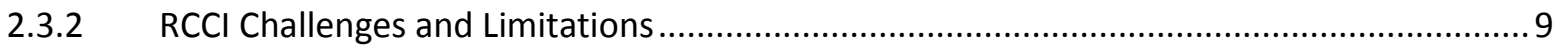

2.3.3 Fuel Property Effects on RCCl Combustion .................................................................. 10

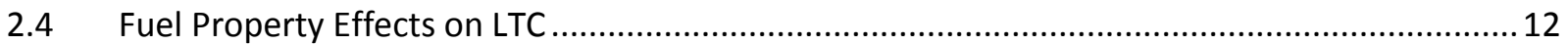

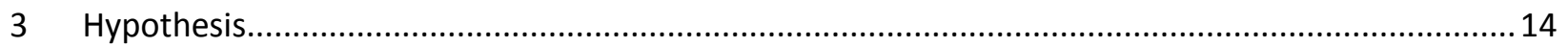

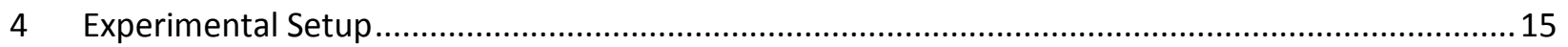

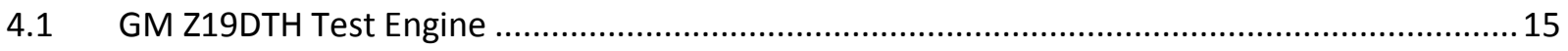

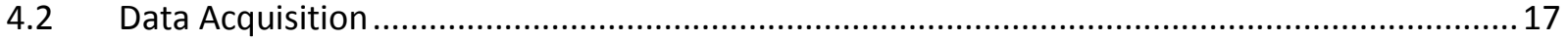

4.3 Exhaust Emissions Measurement ………........................................................................... 17

4.4 Intake Air and Fuel Flow Measurement............................................................................. 18

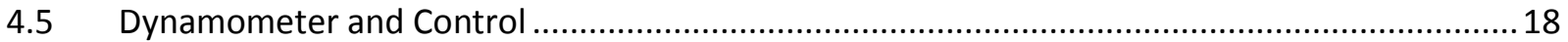

4.6 In-Cylinder Pressure and Combustion Analysis ................................................................ 19

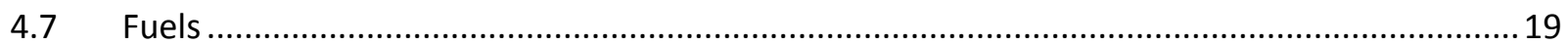

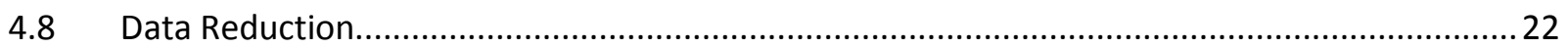

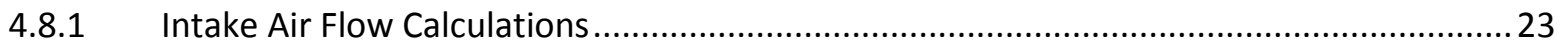

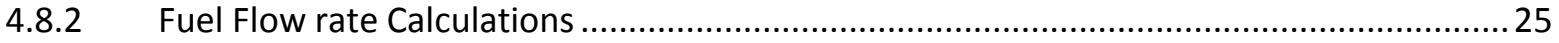

4.8.3 Chemical Balances of Exhaust Emissions to Determine Air-to-Fuel Ratio ..........................25

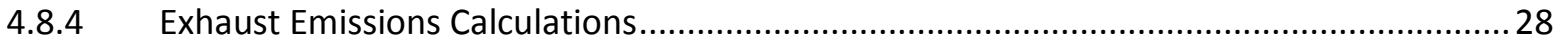

4.8.5 Combustion Efficiency and Brake Thermal Efficiency ..................................................29

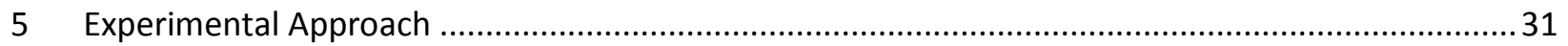

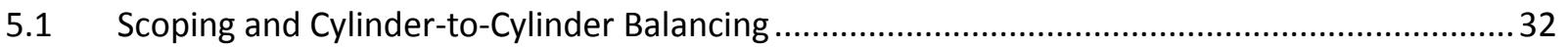

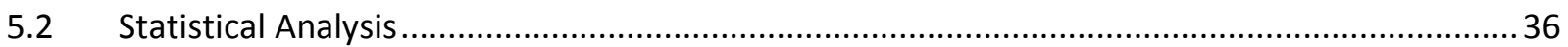

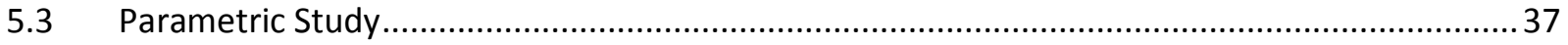


5.3.1 Low Load Operating Condition Parametric Study ..................................................... 37

5.3.2 Low Load Final Test Matrix development and Verification ............................................... 43

5.3.3 High Load Operating Condition Parametric Study ....................................................... 48

5.3.4 High Load Final Test Matrix development ...................................................................... 52

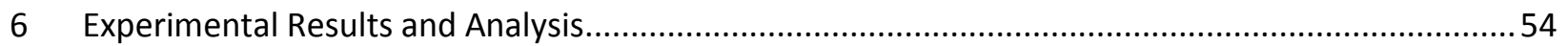

6.1 Ratio of Low Reactivity to High Reactivity Fuel ........................................................................5

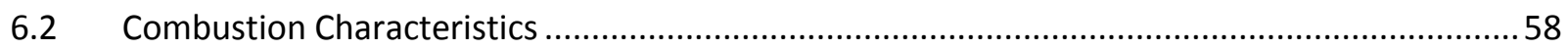

6.2.1 In-Cylinder Pressure and Pressure Rise Rate ................................................................59

6.2.2 Heat Release Rate and its Derivatives .......................................................................... 63

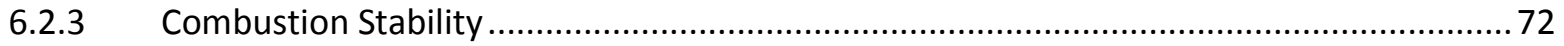

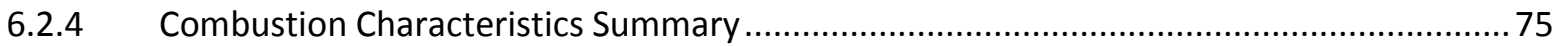

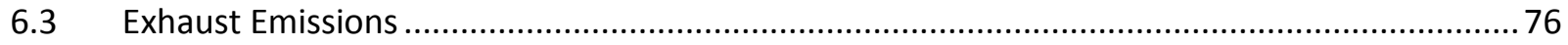

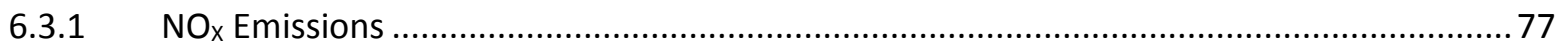

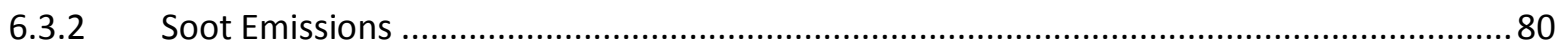

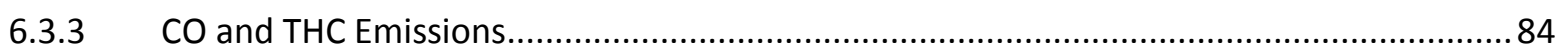

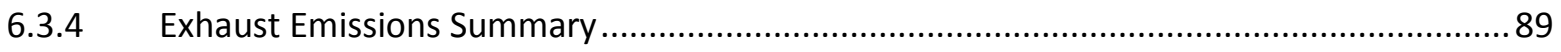

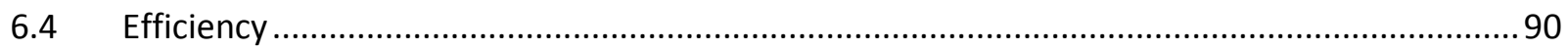

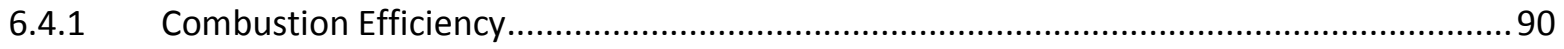

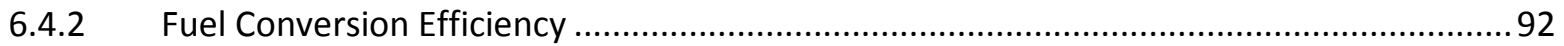

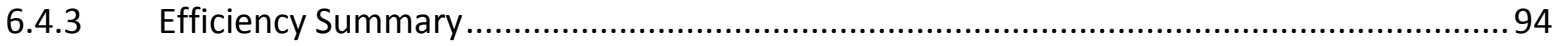

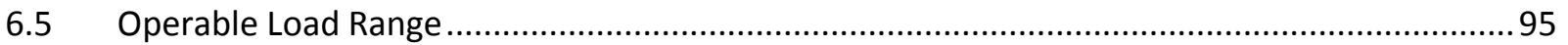

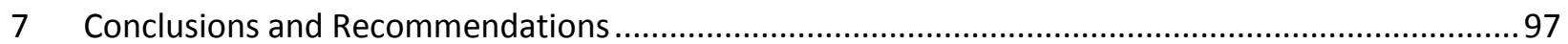

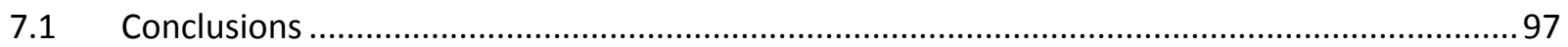

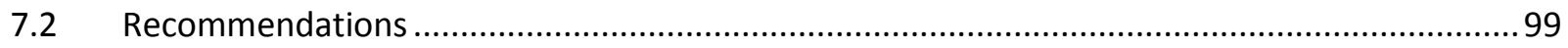

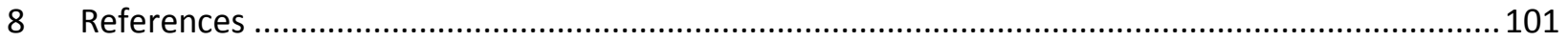




\section{List of Figures}

Figure 1: Conversion Rate versus Exhaust Temperature with an Aged Catalyst for Emissions of a DieselNatural Gas Dual-Fuel Engine [12] ........................................................................................... Figure 2: Local Temperature vs. Local Equivalence Ratio for Advanced Combustion Strategies [13]. ........ 6 Figure 3: Brake Specific NOx Emissions from LTC Operation [14] ....................................................... 7 Figure 4: RCCI Operating Points Explored with GM 1.9L CIDI Engine Fueled with Gasoline and ULSD [8] 10 Figure 5: Soot vs. CN from CRC AVFL-16 Project Test Engine Operating in LTC Mode [14] ...................... 12

Figure 6: GM Z19DTH Light-Duty CI Engine Installed in the CAFEE Test Cell..........................................16

Figure 7: Nozzles for Gaseous Fuel Injectors Installed in the Intake Manifold.........................................16

Figure 8: Remote Mount Gaseous Fuel Injectors Installed on the Test Engine ........................................17

Figure 9: "Raw Lab" CO/CO 2 and THC Analyzers.............................................................................. 18

Figure 10: Target Design Properties of the FACE Diesel Fuels Used in This Study [14] ............................21

Figure 11: T10 versus CN for the FACE and Cert Diesel Fuels ........................................................... 22

Figure 12: RCCI Research Points in Relation to Maximum Torque versus Speed from CDC Operation ..... 31

Figure 13: In-Cylinder Pressure Measurements for Cylinder Balancing Efforts with Chemically Pure $\mathrm{CH}_{4} 33$

Figure 14: In-Cylinder Pressure Measurements for Cylinder Balancing Efforts with CNG ........................34

Figure 15: In-Cylinder Pressure Measurements for $9.25^{\circ}$ PFI Duration with CNG .................................. 35

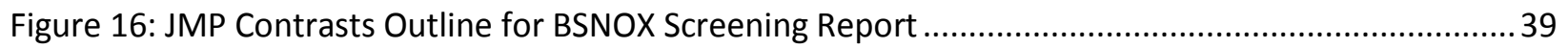

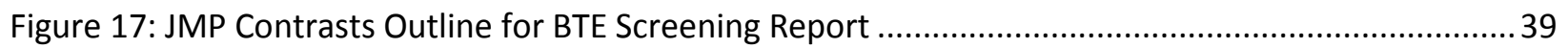

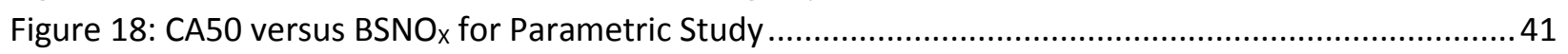

Figure 19: CA50 versus BTE for Parametric Study ............................................................................ 41

Figure 20: JMP Contrasts Outline for CA50 Screening Report with Six Parameters of Interest ................. 43

Figure 21: JMP Contrasts Outline for CA50 Screening Report with Percentage CNG Substituted for PFI

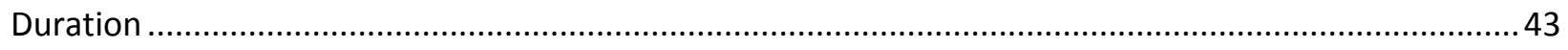

Figure 22: JMP Least Squares Fit Model of BSNOX with IMAP and CA50 versus IMAP and Percentage CNG

Figure 23: JMP Least Squares Fit Model of BTE with IMAP and CA50 versus IMAP and Percentage CNG. 47 Figure 24: Percentage CNG for the Low Load Operating Condition (Varying IMAP and CA50) .................55 Figure 25: Percentage CNG for the High Load Operating Condition (Varying Main DI SOI Timing and

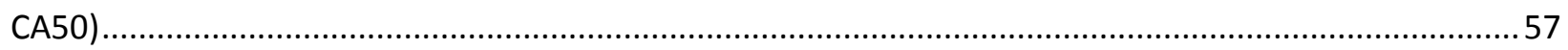

Figure 26: Comparison of In-Cylinder Pressure and HRR for RCCI, CDC, and CDC with EGR ....................58 Figure 27: Maximum In-Cylinder Pressure for the Low Load Operating Condition (Varying IMAP and

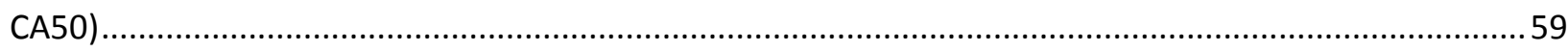

Figure 28: Maximum In-Cylinder Pressure Averages versus T10 for the Low Load Operating Condition.. 60 Figure 29: Maximum In-Cylinder PRR for the Low Load Operating Condition (Varying IMAP and CA50).. 61 Figure 30: Maximum In-Cylinder Pressure for the High Load Operating Condition (Varying Main DI SOI Timing and CA50)

Figure 31: Maximum In-Cylinder PRR for the High Load Operating Condition (Varying Main DI SOI Timing

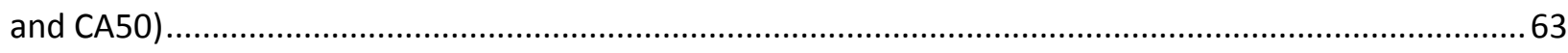

Figure 32: Sample HRR from Each High Reactivity Fuel during Low Load Operation ...............................64 Figure 33: Combustion Duration (CA5 to CA95) at the Low load Operating Condition Ordered by CN (Varying IMAP and CA50)..... .65

Figure 34: Combustion Duration (CA5 to CA95) at the Low load Operating Condition Ordered by T10 (Varying IMAP and CA50). 
Figure 35: Maximum HRR for at the Low load Operating Condition Ordered by CN (Varying IMAP and CA50)

Figure 36: Maximum HRR for at the Low load Operating Condition Ordered by T10 (Varying IMAP and CA50)

Figure 37: Sample HRR from Each High Reactivity Fuel during High Load Operation

Figure 38: Maximum HRR for at the High load Operating Condition Ordered by CN (Varying Main DI SOI

Timing and CA50).....

Figure 39: Maximum HRR for at the High load Operating Condition Ordered by T10 (Varying Main DI SOI

Timing and CA50) 70

Figure 40: Combustion Duration (CA5 to CA95) at the High load Operating Condition Ordered by CN (Varying Main DI SOI Timing and CA50)

Figure 41: Combustion Duration (CA5 to CA95) at the High load Operating Condition Ordered by T10 (Varying Main DI SOI Timing and CA50). . .71

Figure 42: COV of IMEP for the Low Load Operating Condition (Varying IMAP and CA50) ......................72

Figure 43: COV of IMEP versus Percentage CNG for the Low Load Operating Condition

Figure 44: Unfiltered In-Cylinder Pressure Trace for a Cycle with Misfire and a Subsequent Cycle from $\mathrm{RCCl}$ Operation with FACE 6 at the Low Load Operating Condition

Figure 45: COV of IMEP for the High Load Operating Condition (Varying Main DI SOI Timing and CA50) 75 Figure 46: COV of IMEP versus Percentage CNG for the High Load Operating Condition...........................75

Figure 47: NOx Emissions from the Low Load Operating Condition (Varying IMAP and CA50) .................77 Figure 48: NOx Emissions from the Low Load Operating Condition Omitting Low CN Fuels (Varying IMAP and CA50)

Figure 49: NOx Emissions from the High Load Operating Condition (Varying Main DI SOI Timing and CA50)

Figure 50: NOx Emissions from the High Load Operating Condition Ordered by T90 Omitting Low CN

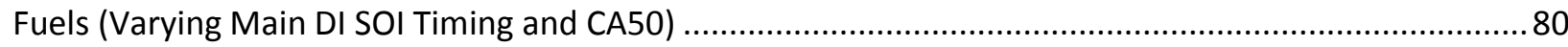

Figure 51: Soot Emissions from the Low Load Operating Condition (Varying IMAP and CA50)................81 Figure 52: Soot Emissions from the Low Load Operating Condition Ordered by T10 (Varying IMAP and CA50)

Figure 53: Soot Emissions from the High Load Operating Condition (Varying Main DI SOI Timing and CA50)

Figure 54: Soot Emissions from the High Load Operating Condition Ordered by T10 (Varying Main DI SOI

Timing and CA50)

Figure 55: CO Emissions from the Low Load Operating Condition (Varying IMAP and CA50) ...................85

Figure 56: THC Emissions from the Low Load Operating Condition (Varying IMAP and CA50) ................86

Figure 57: THC Emissions from the Low Load Operating Condition Ordered by T10 Omitting Low CN Fuels (Varying IMAP and CA50)....

Figure 58: CO Emissions from the High Load Operating Condition (Varying Main DI SOI Timing and CA50)

Figure 59: THC Emissions from the High Load Operating Condition (Varying Main DI SOI Timing and CA50)

Figure 60: Combustion Efficiency at the Low Load Operating Condition (Varying IMAP and CA50) .........90 Figure 61: Combustion Efficiency at the High Load Operating Condition (Varying Main DI SOI Timing and CA50) 
Figure 62: BTE at the Low Load Operating Condition (Varying IMAP and CA50) …..............................92

Figure 63: FCE at the Low Load Operating Condition (Varying IMAP and CA50) ...................................93

Figure 64: FCE at the High Load Operating Condition (Varying Main DI SOI Timing and CA50).................94 


\section{List of Tables}

Table 1: Maximum Global In-Cylinder Temperature from Diesel-Natural Gas Dual-fuel Operation and

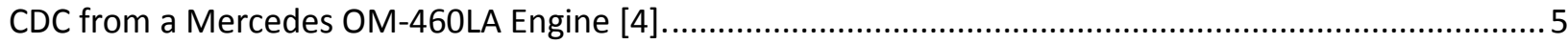

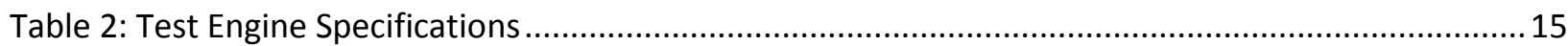

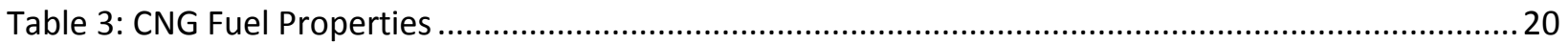

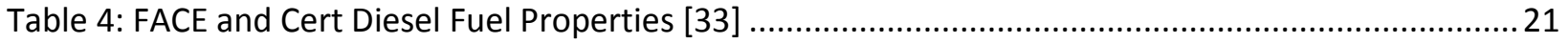

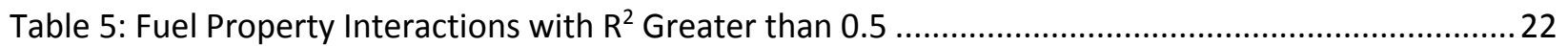

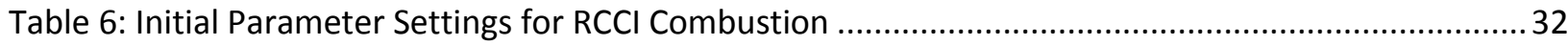

Table 7: Final Injection Duration Coefficients for Cylinder Balancing ....................................................35

Table 8: Input Variable Levels and Values for the Low Load Parametric Study .....................................37

Table 9: L18 Chakravarty DOE Test Matrix for the Low Load Parametric Study ...................................... 38

Table 10: Potentially Influential Parameters from JMP Screening Report Contrasts Outline .................... 40

Table 11: Potentially Influential Parameters from JMP Screening Report Contrasts Outline with CA50 Included

Table 12: Final Test Matrix to Evaluate High Reactivity Fuel Property Effects on RCCI Combustion at the Low Load Operating Condition

Table 13: Set points for Other Parameters of Interest for the Low Load Final Test Matrix ......................45

Table 14: Emissions and Performance Averages, Maximums and Minimums for Pump Diesel and CNG

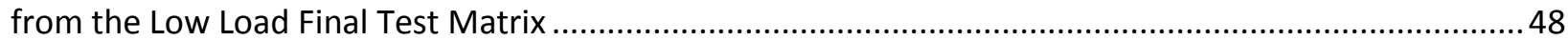

Table 15: Input Variable Levels and Values for the High Load Parametric Study......................................49

Table 16: L18 Chakravarty DOE Test Matrix for the High Load Parametric Study....................................49

Table 17: Potentially Influential Parameters from JMP Screening Report Contrasts Outline ....................51

Table 18: Potentially Influential Parameters from JMP Screening Report Contrasts Outline including Percentage CNG 52

Table 19: Final Test Matrix to Evaluate High Reactivity Fuel Property Effects on RCCl Combustion at the

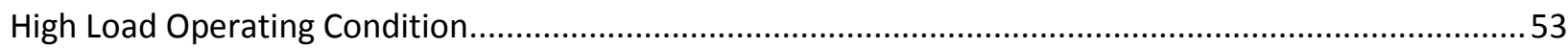

Table 20: Set points for Other Parameters of Interest for the High Load Final Test Matrix ......................53 


\section{List of Abbreviations}

\begin{tabular}{|c|c|}
\hline 2-EHN & 2-Ethylhexyl Nitrate \\
\hline AC & Aromatic Content \\
\hline ASC & Ammonia Slip Catalyst \\
\hline ATDC & After Top Dead Center \\
\hline B20 & Blend of 20 Percent Biodiesel in Diesel \\
\hline BMEP & Brake Mean Effective Pressure \\
\hline BS & Brake Specific \\
\hline BTDC & Before Top Dead Center \\
\hline BTE & Brake Thermal Efficiency \\
\hline $\mathrm{CA}^{\circ}$ & Crank Angle Degree \\
\hline CA50 & Crank Angle Location of 50 Percent Mass Fraction Burned \\
\hline CAFE & Corporate Average Fuel Economy \\
\hline CAFEE & Center for Alternative Fuels, Engines, and Emissions \\
\hline CDC & Conventional Diesel Combustion \\
\hline CFR & Code of Federal Regulations \\
\hline $\mathrm{CH}_{4}$ & Methane \\
\hline $\mathrm{Cl}$ & Compression-Ignition \\
\hline CIDI & Compression-Ignition Direct-Injection \\
\hline $\mathrm{CN}$ & Cetane Number \\
\hline CNG & Compressed Natural Gas \\
\hline $\mathrm{CO}$ & Carbon Monoxide \\
\hline $\mathrm{CO}_{2}$ & Carbon Dioxide \\
\hline cov & Coefficient of Variation \\
\hline CRC & Coordinating Research Council \\
\hline DAQ & Data Acquisition \\
\hline DI & Direct Injection \\
\hline DOC & Diesel Oxidation Catalyst \\
\hline DOE & Design of Experiments \\
\hline DP & Differential Pressure \\
\hline DPF & Diesel Particulate Filter \\
\hline DTBP & Di-Tert-Butyl Peroxide \\
\hline E20 & Blend of 20 Percent Ethanol in Gasoline \\
\hline E85 & Blend of Ethanol in Gasoline up to 85 Percent \\
\hline EGR & Exhaust Gas Recirculation \\
\hline FACE & Fuels for Advanced Combustion Engines \\
\hline FCE & Fuel Conversion Efficiency \\
\hline FID & Flame Ionization Detector \\
\hline GGE & Gasoline Gallon Equivalent \\
\hline GHG & Greenhouse Gas \\
\hline GM & General Motors \\
\hline $\mathrm{H}_{2}$ & Hydrogen \\
\hline $\mathrm{H}_{2} \mathrm{O}$ & Water \\
\hline $\mathrm{HCCl}$ & Homogeneous Charge Compression Ignition \\
\hline
\end{tabular}




\begin{tabular}{|c|c|}
\hline HP & Horsepower \\
\hline HPDI & High Pressure Direct Injection \\
\hline HRR & Heat Release Rate \\
\hline ICE & Internal Combustion Engine \\
\hline IMAP & Intake Manifold Air Pressure \\
\hline IMAT & Intake Manifold Air Temperature \\
\hline IMEP & Indicated Mean Effective Pressure \\
\hline LFE & Laminar Flow Element \\
\hline LTC & Low Temperature Combustion \\
\hline LTFT & Low Temperature Fischer Tropsch \\
\hline LTHR & Low Temperature Heat Release \\
\hline MFB & Mass Fraction Burned \\
\hline MFC & Mass Flow Controller \\
\hline $\mathrm{N}_{2}$ & Nitrogen \\
\hline NDIR & Non-Dispersive Infrared \\
\hline $\mathrm{NI}$ & National Instruments \\
\hline NMEP & Net Mean Effective Pressure \\
\hline NO & Nitric Oxide \\
\hline NO & Nitrogen Dioxide \\
\hline $\mathrm{NO}_{\mathrm{x}}$ & Oxides of Nitrogen \\
\hline ORNL & Oak Ridge National Laboratory \\
\hline $\mathrm{PCCl}$ & Premixed Charge Compression Ignition \\
\hline $\mathrm{PFI}$ & Port Fuel Injection \\
\hline PID & Proportional Integral Derivative \\
\hline PM & Particulate Matter \\
\hline ppm & Parts per Million \\
\hline PRF & Primary Reference Fuel \\
\hline PRR & Pressure Rise Rate \\
\hline $\mathrm{RCCl}$ & Reactivity Controlled Compression Ignition \\
\hline RPM & Revolutions per Minute \\
\hline RTD & Resistive Temperature Detector \\
\hline SCR & Selective Catalytic Reduction \\
\hline SG & Specific Gravity \\
\hline SI & Spark Ignited \\
\hline SOI & Start of Injection \\
\hline $\mathrm{SO}_{\mathrm{x}}$ & Oxides of Sulfur \\
\hline T10 & 10 Percent Distillation Temperature \\
\hline T90 & 90 Percent Distillation Temperature \\
\hline TDC & Top Dead Center \\
\hline THC & Total Hydrocarbons \\
\hline ULSD & Ultra-Low Sulfur Diesel \\
\hline VGT & Variable Geometry Turbocharger \\
\hline VOC & Volatile Organic Compound \\
\hline WVU & West Virginia University \\
\hline
\end{tabular}




\section{Introduction}

The progression of internal combustion engine (ICE) technology over the last five decades has largely been driven by federal and state legislations. The implementation of these regulations has imposed limits on specific compounds emitted by ICEs through the exhaust tailpipe and crankcase vent. Six criterion pollutants were identified in the 1970 Clean Air Act, and subsequent revisions, as affecting human health and included particle matter (PM), ground-level ozone, carbon monoxide (CO), oxides of sulfur $\left(\mathrm{SO}_{\mathrm{x}}\right)$, oxides of nitrogen $\left(\mathrm{NO}_{\mathrm{x}}\right)$, and lead. Note that ground level ozone is formed by chemical reactions of $\mathrm{NO}_{\mathrm{x}}$ and volatile organic compounds (VOCs), such as total hydrocarbon (THC) emissions from ICEs, in the presence of sunlight. Emissions control devices, developed to mitigate the discharge of such pollutants, have added additional costs and complexities to ICEs. When initially implemented in the 1970s, many of these devices reduced performance while increasing fuel consumption [1]. These drawbacks have driven research activities to develop technologies that reduce engine-out exhaust emissions and improve fuel efficiency. By the 1990s, automobiles on average consumed less than half the fuel that they did in the 1970s [1]. Recent federal legislation, such as more stringent corporate average fuel economy (CAFE) standards, present and future federal greenhouse gas (GHG) emissions regulations, and rising fuel costs have also driven research organizations and manufacturers to develop strategies and technologies that increase overall vehicular fuel efficiency.

Compression-ignition (Cl) engines have historically offered superior fuel efficiency over spark-ignited (SI) engines. Fuel efficiency shortcomings of SI engines can be partially attributed to pumping losses encountered by the use of a throttling valve to modulate the mass flow of air and subsequently control engine output power. Additionally, SI engines equipped with three-way catalysts must operate near a stoichiometric air-to-fuel ratio for this catalyst to convert $\mathrm{CO}, \mathrm{THC}$, and $\mathrm{NO}_{\mathrm{x}}$ emissions to carbon dioxide $\left(\mathrm{CO}_{2}\right)$, water $\left(\mathrm{H}_{2} \mathrm{O}\right)$ and nitrogen $\left(\mathrm{N}_{2}\right)$. Cl engines are conventionally un-throttled (although some modern $\mathrm{Cl}$ engines utilize throttles for emissions control purposes) and operate at a globally lean air-to-fuel ratio for much of their operating range. $\mathrm{Cl}$ engines also tend to have comparatively high compression ratios in the range of 12 to 20:1, which can allow for higher mean effective pressures and subsequently greater overall engine efficiency, whereas $\mathrm{SI}$ engine compression ratios are limited by auto-ignition or knock to the range of 7 to $10: 1$.

Although $\mathrm{Cl}$ engines can offer fuel efficiency benefits over $\mathrm{SI}$ engines they are plagued by soot (a significant component of particulate matter) and $\mathrm{NO}_{\mathrm{x}}$ emissions requiring aftertreatment solutions more extensive (circa 2010) than three-way catalysts in order to meet current federal and state emissions regulations. For example, typical post 2010 model year on-road heavy-duty diesel engine exhaust aftertreatment systems consist of a diesel oxidation catalyst (DOC) to oxidize CO and THC, a diesel particulate filter (DPF) to trap and oxidize PM, a selective catalytic reduction (SCR) system that relies on a urea based solution (commonly referred to as diesel exhaust fluid) to reduce $\mathrm{NO}_{\mathrm{x}}$, and an ammonia slip catalyst (ASC) to clean up ammonia from the SCR urea and $\mathrm{NO}_{x}$ reaction. Many light-duty and medium-duty diesel vehicles are also using DOC, DPF, and SCR technology to meet federal and state exhaust emissions standards. Due to their complexity, exhaust aftertreatment systems for modern $\mathrm{Cl}$ engines are significantly more expensive to manufacture and occupy more volume on the vehicle compared to three-way catalyst technology used for SI engines. 
From 2013 to January of 2016, petroleum prices have fluctuated significantly. The national average for a gallon of on-road ultra-low sulfur diesel (ULSD) was \$2.59 and \$2.23 in October of 2015 and January of 2016 , respectively, while the national average for a gallon of regular grade gasoline was $\$ 2.35$ and $\$ 1.98$ in October of 2015 and January of 2016, respectively [2]. However, compressed natural gas (CNG) remained at $\$ 2.09$ on a gasoline gallon equivalent (GGE) basis in October of 2015 and January of 2016 [2]. The price stability of CNG over time is a function of the abundant domestic natural gas supply and demonstrates the independence of natural gas prices from petroleum prices. Although ULSD and gasoline were comparable in price to CNG in October of 2015 and January of 2016, its price stability makes it an alluring fuel with regards to planning future costs, especially for commercial applications. Two options exist to use natural gas in reciprocating ICEs. SI natural gas engines are one option, however they suffer from the same fuel economy drawbacks that SI gasoline engines do when compared to $\mathrm{Cl}$ engines. The other option is dual-fuel $\mathrm{Cl}$ technology. Dual-fuel $\mathrm{Cl}$ engines are able to combust high octane number fuels (e.g. natural gas or gasoline) without a spark plug by utilizing a reduced quantity of high $\mathrm{CN}$ fuel (diesel) as the ignition source and the high octane number fuel as the primary energy source. Dual-fuel technology has the capability of retaining high thermal efficiencies associated with diesel-fueled engines while also utilizing high octane fuels such as gasoline or natural gas, although conventional dual-fuel $\mathrm{Cl}$ engines still require extensive exhaust aftertreatment systems due to their lean operation.

Literature and research results presented herein make the case for the use of CNG and diesel dual-fuel technology that can reduce engine out $\mathrm{NO}_{x}$ and soot emissions to levels that could alleviate the need or reduce the size of costly SCR and DPF exhaust aftertreatment technology while retaining or even improving the fuel efficiency that is associated with $\mathrm{Cl}$ engines. This technology is called reactivity controlled compression ignition (RCCl). In this study, diesel (high reactivity) fuel property effects and ignition characteristics on diesel-natural gas $\mathrm{RCCl}$ are investigated to address challenges with this technology and provide an efficient means of utilizing natural gas as a transportation fuel while potentially reducing the cost and complexity of $\mathrm{Cl}$ engine exhaust aftertreatment systems. 


\section{Literature Review}

Increased production of domestic natural gas in recent years has allowed natural gas prices to remain relatively low even as petroleum prices have fluctuated. This has encouraged engine manufacturers and aftermarket outfitters to develop and modify $\mathrm{Cl}$ engines to substitute a portion of the diesel fuel energy with natural gas or other high octane number fuels. In many of these dual-fuel engines, the high octane fuel is introduced into the intake manifold prior to the intake valve. Some aftermarket dual-fuel kits use a mechanical or electronically controlled mixer as a single point of injection while others use multiple electronic injectors to sequentially meter fuel to each cylinder. More advanced fuel delivery options exist specifically for natural gas dual-fuel applications, such as Westport's high pressure direct injection (HPDI), where both the natural gas and diesel fuel are injected directly into the cylinder by means of a high pressure injector [3]. For the majority of modern dual-fuel engines, a reduced quantity of high CN fuel, most commonly on-road ULSD, is injected directly into the cylinder through the factory diesel injectors. The timing of this injection and subsequent combustion of the high $\mathrm{CN}$ fuel is what dictates the timing or phasing of the combustion of the high octane number fuel and air mixture.

\subsubsection{Dual-Fuel Substitution Ratio}

The ratio of high $\mathrm{CN}$ fuel displaced by high octane fuel in a dual-fuel engine is often referred to as the substitution ratio and is dependent on several factors. The maximum substitution ratio a dual-fuel engine can achieve is primarily dependent on combustion chamber geometry, engine speed, and engine load. For commercial applications, substitution ratio can be limited by engine knock, degraded combustion efficiency and increases in regulated exhaust emissions. The combustion chamber geometry, more specifically compression ratio, and mixing characteristics of an engine, directly influences engine knock and combustion efficiency. High substitution ratios at elevated engine speed and load conditions may be hindered by high in-cylinder pressure, pressure rise rate (PRR), and engine knock $[4,5]$. Therefore, modern diesel engine compression ratios, generally in the range of 12:1 to 20:1, may limit substitution ratios. Reducing the compression ratio of a conventional diesel engine can allow for higher substitution ratios, but may simultaneously decrease fuel conversion efficiency (FCE) [6]. The mixing characteristics and combustion chamber geometry directly affect the combustion efficiency of the natural gas and air mixture because of their influence on flame propagation and the ability of the flame front to reach fuel near the walls and crevices of the cylinder and piston [7]. Engine speed and load affect allowable substitution ratios because of their direct effect on in-cylinder mixing characteristics, in-cylinder temperature and pressures. Substitution ratios of natural gas at low engine speed and load conditions are limited by increased $\mathrm{CO}$ and THC engine out exhaust emissions, degraded combustion efficiency, and increased engine instabilities $[7,8]$. Low in-cylinder temperatures and pressures at these conditions limit flame propagation and prevent the natural gas and air mixture from combusting.

\subsubsection{Emissions from Dual-Fuel Compression-Ignition Engines}

Dual-fuel diesel-natural gas engines have the potential to emit less $\mathrm{NO}_{x}, \mathrm{CO}_{2}$ and $\mathrm{PM}$ emissions than conventional diesel fueled engines, but at the expense of elevated CO and THC (mainly in the form of methane) emissions. These outcomes are heavily dependent on combustion phasing and other engine operating characteristics.

Thermal $\mathrm{NO}_{x}$ formation is the primary mechanism in which $\mathrm{NO}_{x}$ is formed in the cylinder of an ICE [1]. In the presence of high temperatures and oxygen, $\mathrm{N}_{2}$ disassociates to form nitric oxide (NO), which can 
subsequently further react to form nitrogen dioxide $\left(\mathrm{NO}_{2}\right)$. From a regulatory perspective, $\mathrm{NO}_{x}$ is defined as the summation of $\mathrm{NO}$ and $\mathrm{NO}_{2}$. Substituting natural gas for diesel can reduce thermal $\mathrm{NO}_{x}$ formation due to several reasons. Natural gas consists predominantly of methane $\left(\mathrm{CH}_{4}\right)$ which has a lower adiabatic flame temperature than diesel [9] resulting in a lower overall combustion temperature which is the underlying basis for thermal $\mathrm{NO}_{\mathrm{x}}$ formation. Combustion temperatures can also be reduced by displacing air, and thus oxygen, entering the cylinder. This is accomplished in a typical diesel-natural gas dual-fuel engine by injecting natural gas into the intake manifold. The natural gas and air mixture entering the cylinder of a dual fuel engine has a higher specific heat than air alone and thus absorbs more heat during the compression process, which reduces the charge temperature prior to combustion [10]. Furthermore, it has been documented that during conventional diesel combustion (CDC) substantial NO formation occurs when local air-to-fuel ratios are near stoichiometric [11]. This occurs as the diesel jet mixes with the surrounding air and burns. By injecting natural gas into the intake manifold, it has more time to mix with the incoming air at a lean air-to-fuel ratio and thus reduces the prevalence of locally stoichiometric air-to-fuel ratios in the cylinder, which subsequently reduces conditions that provide substantial NO formation.

Because natural gas primarily consists of $\mathrm{CH}_{4}$, which has less carbon molecules per unit of energy compared to diesel, it has a theoretically lower propensity to form soot or PM during combustion. Thus, substituting diesel with natural gas can reduce PM emissions. Furthermore, in a dual-fuel diesel-natural gas engine (in which the natural gas is port injected), the natural gas has more of an opportunity to mix homogeneously with the air prior to combustion resulting in less locally rich regions in which PM is formed. The combustion of this more homogeneous mixture of air and natural gas offsets the amount of diffusion flame and mixing controlled combustion, which is the predominant source of PM formation during CDC [11]. On the contrary, the addition of natural gas into the intake manifold displaces a portion of the air that would enter the combustion chamber during CDC and thus may result in increased PM due to an overall lower air-to-fuel ratio, especially in the region surrounding the diesel injection jet where a substantial portion of the total PM is formed [11]. Additionally, combustion phenomena that reduce $\mathrm{NO}_{x}$ emissions typically increase $\mathrm{PM}$ emissions because reducing combustion temperatures to limit thermal $\mathrm{NO}_{x}$ formation also reduces the oxidation of THC and PM. Hardware and control strategy limitations of aftermarket dual-fuel diesel-natural gas retrofits can also create situations where the PM emissions during dual fuel operation are greater than CDC. Due to the location of natural gas injectors in the intake manifold versus diesel injectors in the cylinder itself, changes in the desired flow rate of natural gas into the cylinder reach the cylinder later than direct diesel injections allowing for instances where the global air-to-fuel ratio becomes rich and PM formation increases, such as during sudden accelerations or decelerations. Residual natural gas in the intake manifold and runners may also cause undesired air-to-fuel ratios in upcoming combustion cycles. In conclusion, there exists a potential to reduce PM emissions with dual-fuel natural-gas operation, but the outcomes are very dependent on the technology that is utilized.

In contrast to CDC, dual-fuel engines typically generate significantly greater amounts of THC and CO emissions. A major source of this phenomenon is the lower combustion temperatures exhibited by dual-fuel diesel-natural gas combustion versus CDC. Lower combustion temperatures limit the oxidation of $\mathrm{CO}$ and THC compounds [10]. A study performed at West Virginia University (WVU) on a 12.8 liter 2005 Mercedes OM-460LA diesel engine that was retrofitted to operate on dual-fuel (diesel and natural gas) demonstrated lower maximum global in-cylinder temperatures compared to CDC as shown in Table 
1. These temperatures were derived from in-cylinder pressure measurements and represent the average or global temperature of the contents of the cylinder.

Table 1: Maximum Global In-Cylinder Temperature from Diesel-Natural Gas Dual-fuel Operation and CDC from a Mercedes OM-460LA Engine [4].

\begin{tabular}{|c|c|c|c|}
\hline \multicolumn{4}{|c|}{ Maximum Global In-Cylinder Temperature } \\
\hline Load (\%) & CDC (K) & Dual-fuel (K) & \% Change \\
\hline 25 & 1250 & 1060 & $-15.2 \%$ \\
\hline 50 & 1440 & 1280 & $-11.1 \%$ \\
\hline 75 & 1550 & 1430 & $-7.74 \%$ \\
\hline 100 & 1640 & 1550 & $-5.49 \%$ \\
\hline
\end{tabular}

Another contributor to increased THC and CO emissions from dual-fuel combustion is the displacement of oxygen by natural gas injected into the intake manifold and the resulting decrease in air-to-fuel ratio compared to $\mathrm{CDC}$ if no changes are made to provide more air. $\mathrm{CO}$ and THC emissions have a direct dependence on the amount of oxygen available for combustion. As the air and fuel mixture becomes richer, $\mathrm{CO}$ and THC emissions increase exponentially [1]. Additionally, converting a direct injection (DI) diesel engine to dual-fuel causes it to operate more like a spark ignited engine and unburned hydrocarbons in the crevices become another major source of THC emissions. Elevated THC emissions from dual-fuel diesel-natural gas combustion will consist predominantly of $\mathrm{CH}_{4}$, which can be especially problematic with regards to meeting recently introduced greenhouse gas emissions. This problem is further complicated by the relatively high exhaust temperatures necessary for oxidation catalysts to efficiently convert $\mathrm{CH}_{4}$ to $\mathrm{CO}_{2}$ and $\mathrm{H}_{2} \mathrm{O}$ versus the temperatures required for higher order hydrocarbons and $\mathrm{CO}$ [12]. As illustrated in Figure 1, oxidation of $\mathrm{CH}_{4}$ begins around $350{ }^{\circ} \mathrm{C}$, however $80 \%$ conversion efficiency is not achieved until temperatures over $450^{\circ} \mathrm{C}$ are present. To initiate oxidation and achieve higher conversion efficiency at lower exhaust temperatures more expensive catalysts materials, higher fuel consumption, or active heating may be necessary which can increase the cost and complexity of the engine and the aftertreatment system.

\section{CONVERSION, \%}

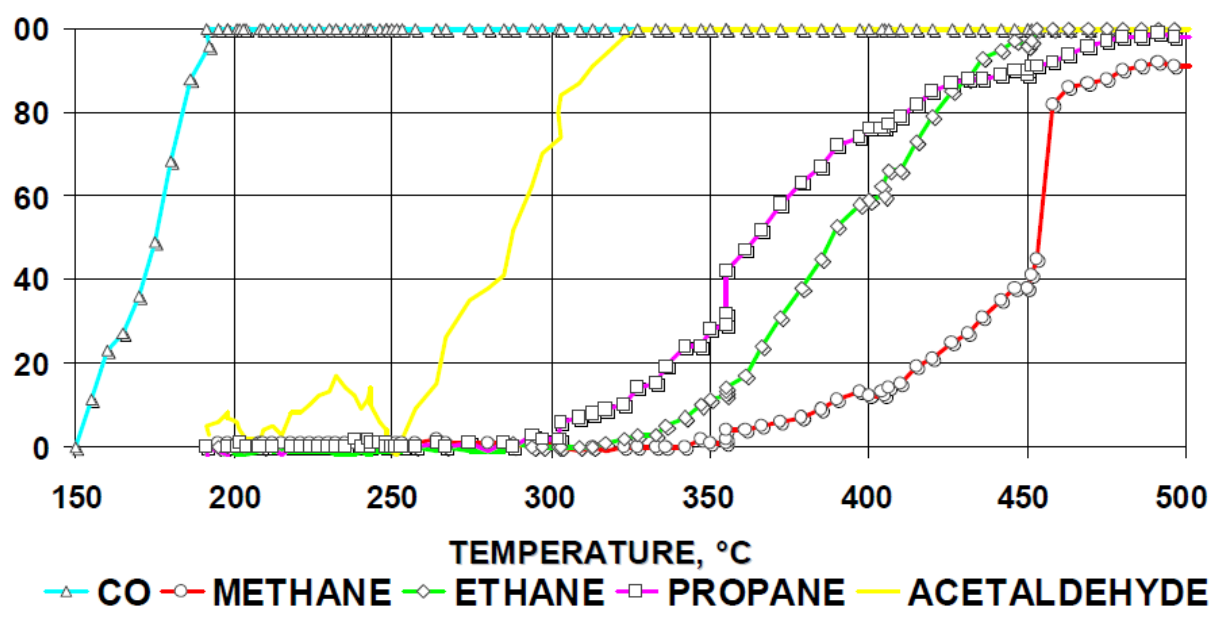

Figure 1: Conversion Rate versus Exhaust Temperature with an Aged Catalyst for Emissions of a DieselNatural Gas Dual-Fuel Engine [12] 


\subsection{Low Temperature Combustion}

Low temperature combustion (LTC) is an advanced combustion strategy that can simultaneously reduce soot and $\mathrm{NO}_{\mathrm{x}}$ emissions when compared to conventional combustion strategies. Several additional advanced combustion strategies exist, which all share common attributes with LTC, including homogeneous charge compression ignition $(\mathrm{HCCl})$, premixed charge compression ignition $(\mathrm{PCCl})$ and $\mathrm{RCCl}$. The underlying theories of these advanced combustion strategies are similar; achieve a homogeneous or near-homogenous air and fuel mixture that is compression ignited spontaneously. This approach limits in-cylinder temperatures due to reduced flame propagation and reduces locally rich regions in the combustion chamber due to the homogenized air and fuel mixture. Figure 2 provides a comparison of some of these advanced and conventional combustion strategies on a plot of local equivalence ratio versus local temperature with shaded contour regions indicating soot and NO formation intensities.

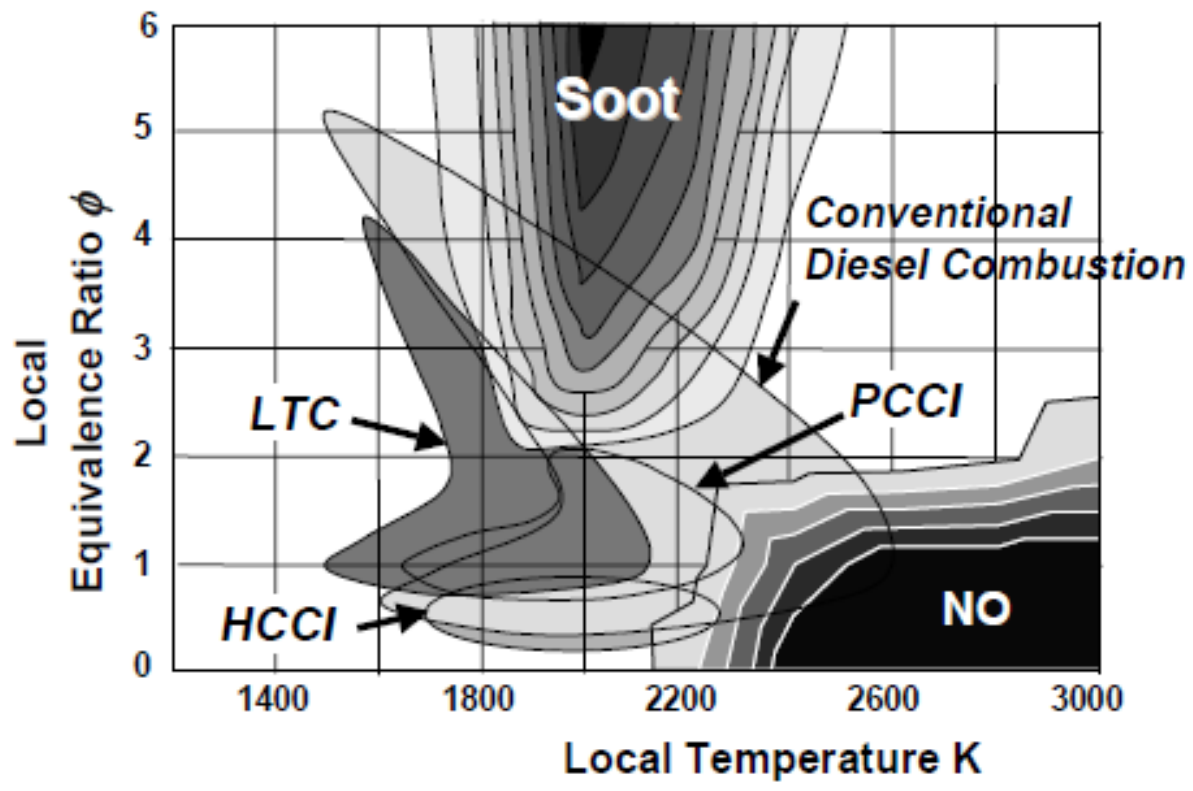

Figure 2: Local Temperature vs. Local Equivalence Ratio for Advanced Combustion Strategies [13].

As shown in Figure 2, CDC occupies the largest operating region on the plot, with a modest area of its operating envelope falling within the soot and $\mathrm{NO}$ formation regions. Conversely, $\mathrm{LTC}, \mathrm{PCCl}$, and $\mathrm{HCCl}$ have smaller operating regions that generally fall outside of the heavy soot and NO formation zones. As a result, it can be difficult to achieve these advanced combustion strategies for certain parts of an engine operating map such as low speed, low load and high speed, high load conditions. Additionally, engine control strategies, and in some instances engine hardware, must be altered to achieve these combustion regimes.

\subsubsection{Engine Control Changes to Achieve LTC}

Modern electronically controlled DI, Cl engines offer the ability to control various facets of the fuel injection, exhaust gas recirculation (EGR) system, intake manifold air pressure (IMAP) via variable geometry turbochargers (VGTs), and in cylinder mixing characteristics through the use of swirl valves. With regards to direct fuel injection, which is typically used for diesel engines, start of injection (SOI) 
timing plays a significant role in the achievement of advanced combustion regimes. Advancement of injection timing during CDC advances combustion phasing and thus increases in-cylinder pressures and temperatures resulting in greater thermal $\mathrm{NO}_{x}$ formation. In contrast, many advanced combustion regimes often rely on advanced injection timing to provide more time for the air and fuel mixture to approach homogeneity prior to combustion. These early injections of fuel are often performed in conjunction with other control strategy modifications to retain proper combustion phasing, such as elevated EGR levels to limit excessive in-cylinder pressures and temperatures yet provide the time necessary for enhanced mixing of the air and fuel mixture. EGR limits in-cylinder pressures and temperatures because the exhaust gas that is reintroduced into the intake stream of the engine is lower in oxygen content than fresh air and acts as a diluent that slows the rate of combustion. An example is demonstrated in Figure 3, which presents brake specific $\mathrm{NO}_{\mathrm{x}}$ emissions for advanced injection timings as the EGR rate increases [14]. This data was collected from LTC operation of a multi-cylinder light-duty $\mathrm{Cl}$ engine. SOI timing was altered to keep the location of 50 percent mass fraction burned (MFB) (CA50) constant as EGR rate varied. Figure 3 demonstrates that with increased EGR, SOI timing can be advanced and a reduction in NOx emissions can be achieved for LTC operation. This trend is in opposition to $\mathrm{CDC}$ norms where the advancement of $\mathrm{SO}$ timing results in increased $\mathrm{NO}_{\mathrm{x}}$ emissions due to higher in-cylinder pressures and subsequently in-cylinder temperatures.

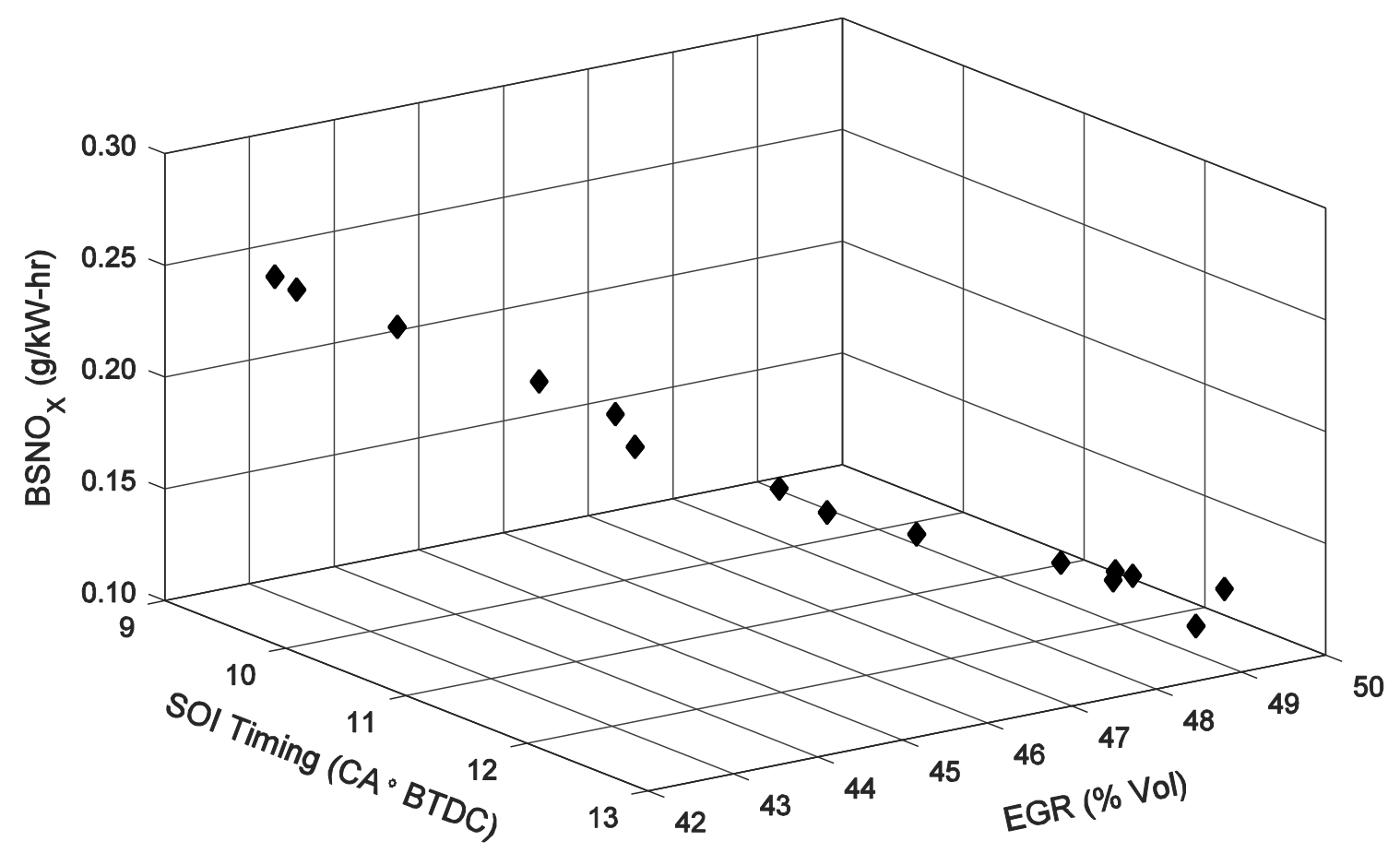

Figure 3: Brake Specific NOx Emissions from LTC Operation [14]

DI common rail fuel injection equipped engines retain the ability to adjust the fuel injection pressure for performance and exhaust emissions by increasing or decreasing the pressure in the common rail. This can have a profound effect on air and fuel mixing, and thus engine performance and emissions characteristics. Increasing the rail pressure and subsequent injection pressure results in earlier 
atomization of the fuel and reduced delivery time that encourages a more homogenous air and fuel mixture within the cylinder. These systems are also capable of providing multiple injections per combustion cycle, which can be used to reduce in-cylinder pressures and PRR as well as improve mixing of the air and fuel. These technologies are essential to facilitate LTC.

\subsection{Reactivity Controlled Compression-Ignition}

$\mathrm{RCCl}$ is a form of dual-fuel technology that incorporates LTC to reduce NOx and soot emissions while retaining high thermal efficiencies [15]. RCCl focuses on in-cylinder mixing and exploitation of the reactivity difference between a high reactivity fuel (high $\mathrm{CN}$ ) and a low reactivity fuel (high octane number) to control combustion phasing. Dual-fuel combustion in the conventional sense relies predominantly on the injection timing and ignition of the high reactivity (high $\mathrm{CN}$ ) fuel to control the combustion of the air and low reactivity fuel mixture.

\subsubsection{Early RCCI Research}

Researchers at UW-Madison initially used a KIVA-CHEMKIN numerical analysis and a reduced primary reference fuel (PRF) mechanism to explore optimal gasoline and diesel fuel blends and EGR rates on LTC regimes [16]. They concluded that neither neat diesel (high reactivity fuel) nor neat gasoline (low reactivity fuel) operation could provide optimal fuel consumption. Additionally, the fuel reactivity required for optimal fuel consumption decreased with engine load. Engine experiments performed on a Caterpillar single-cylinder oil test engine confirmed these results. Utilizing port-fueled gasoline injection and early direct diesel injection, the researchers were able to demonstrate approximately $.01 \mathrm{~g} / \mathrm{kW}-\mathrm{hr}$ and $.008 \mathrm{~g} / \mathrm{kW}$-hr of engine out indicated specific $\mathrm{NO}_{\mathrm{x}}$ and soot emissions, respectively, while achieving 50 percent indicated thermal efficiency at an indicated mean effective pressure (IMEP) of 11 bar. It was noted that these exhaust emissions levels are below the 2010 heavy-duty diesel engine exhaust emissions standards for $\mathrm{NO}_{\mathrm{x}}$ and $\mathrm{PM}$ but it is important to consider that they are indicated specific results versus the standards, which are brake-specific, and they were obtained at steady-state operation whereas the emissions standards include both transient and steady state operation tests. Also, note that soot is a component of PM and the addition of the soluble organic fraction contribution to PM will result in a PM measurement that is greater than a soot measurement from the same exhaust stream. Nonetheless, the low $\mathrm{NO}_{x}$ and soot measurements in conjunction with high thermal efficiency and relatively high load compared to other advanced combustion strategies, provided encouragement to continue $\mathrm{RCCl}$ research and development.

$\mathrm{RCCl}$ combustion has been further studied by researchers at UW-Madison as well as other organizations. Alternative engine applications including multi-cylinder experiments as well as parametric studies and combustion chamber geometry experiments have been performed by different researchers $[17,18,19$, 20, 21, 22]. Dunbeck, et al. implemented RCCl, with gasoline and diesel, on an air-cooled high-speed, DI diesel generator. Their results demonstrated significant reductions in soot at high load operation, although low load operation resulted in decreased combustion efficiency and excessive THC and CO emissions compared to baseline diesel operation [17]. Curran, et al. investigated gasoline and diesel $\mathrm{RCCl}$ operation on a 1.9 liter General Motors (GM) light-duty diesel engine operating at 2300 revolutions per minute (RPM) and 5.5 bar net mean effective pressure (NMEP) [18]. They observed a greater than 90 percent reduction in $\mathrm{PM}$ and $\mathrm{NO}_{\mathrm{x}}$ emissions while achieving similar, but slightly improved, brake and indicated thermal efficiencies compared to CDC. CO and THC emissions increased by a minimum of 194 and 467 percent, respectively, while exhaust temperatures decreased from $412^{\circ} \mathrm{C}$ to a minimum of 235 
${ }^{\circ} \mathrm{C}$. It was also concluded that intake charge temperature was a critical combustion phasing parameter that dictated the operable gasoline-to-diesel fuel ratio.

\subsubsection{RCCI Challenges and Limitations}

Although $\mathrm{RCCl}$ combustion has been proven to reduce $\mathrm{NO}_{\mathrm{x}}$ and soot emissions while achieving high thermal efficiencies compared to CDC, it does have shortcomings similar to other LTC strategies. As noted above, it has been demonstrated that $\mathrm{THC}$ and $\mathrm{CO}$ emissions from $\mathrm{RCCl}$ can be orders of magnitude greater than THC and CO emissions from $\operatorname{CDC}[17,18,23]$. Additionally, exhaust temperatures at low load conditions have been observed to be less than $200^{\circ} \mathrm{C}$. Coupling low exhaust temperatures with elevated THC and CO emissions creates a challenge for conventional DOCs [24]. In that study, Prikhodko, et al. observed that no THC or CO conversion occurred at exhaust temperatures below $200{ }^{\circ} \mathrm{C}$ during $\mathrm{RCCl}$ combustion for several DOCs tested, whereas during CDC operation, 80 and 100 percent conversion of THC and CO emissions, respectively, was achieved at an exhaust temperature of $190{ }^{\circ} \mathrm{C}$. The authors attributed these results to the elevated levels of THC and CO emissions during $\mathrm{RCCl}$ combustion as well as the influence and incompatibility of different chemical compositions of RCCI exhaust compared to CDC exhaust on the DOC.

Limitations to the operable load range are also faced when pursuing RCCl. Low load RCCl operation can lead to increased combustion instabilities, particularly for multi-cylinder applications [23], as well as decreased combustion efficiency [17]. High load operation is limited by potentially detrimental incylinder PRRs, which are generally considered to be above 10 bar per crank angle degree $\left(C A^{\circ}\right)[19,25]$. Nieman, et al. [25] attributed these high PRRs to the premixed charge nature of RCCl combustion. Curran, et al. developed an $\mathrm{RCCl}$ engine calibration and documented its operable load range, efficiency and emissions characteristics utilizing a GM 1.9 liter compression-ignition direct-injection (CIDI) engine [8]. That engine was the same model but later model year than the engine used at WVU for current and previous advanced combustion experiments. The experiments used certification grade gasoline (UTG96) and diesel (ULSD) fuel and the engine was modified with purely concave bowl type pistons that lowered its compression ratio from 17:1 to 15:1. Figure 4 displays the operable RCCI load range that was observed by Curran, et al. The black dots represent actual speed and load conditions that were tested, while the red lines are interpolations of the maximum or minimum load. The load range was constrained by a maximum in-cylinder PRR of $10 \mathrm{bar} / \mathrm{CA}^{\circ}$ on the upper extreme, and exhaust CO emissions of 5,000 parts per million (ppm) on the lower extreme. At an engine speed of 2000 RPM the maximum load decreased from nearly 18 bar to 6 bar brake mean effective pressure (BMEP) for RCCI operation compared to CDC operation. The minimum load increased from less than 1 bar BMEP for CDC to an interpolated 1 bar BMEP for RCCI. In fact, the minimum RCCl load that was actually tested was nearly 2 bar BMEP. 


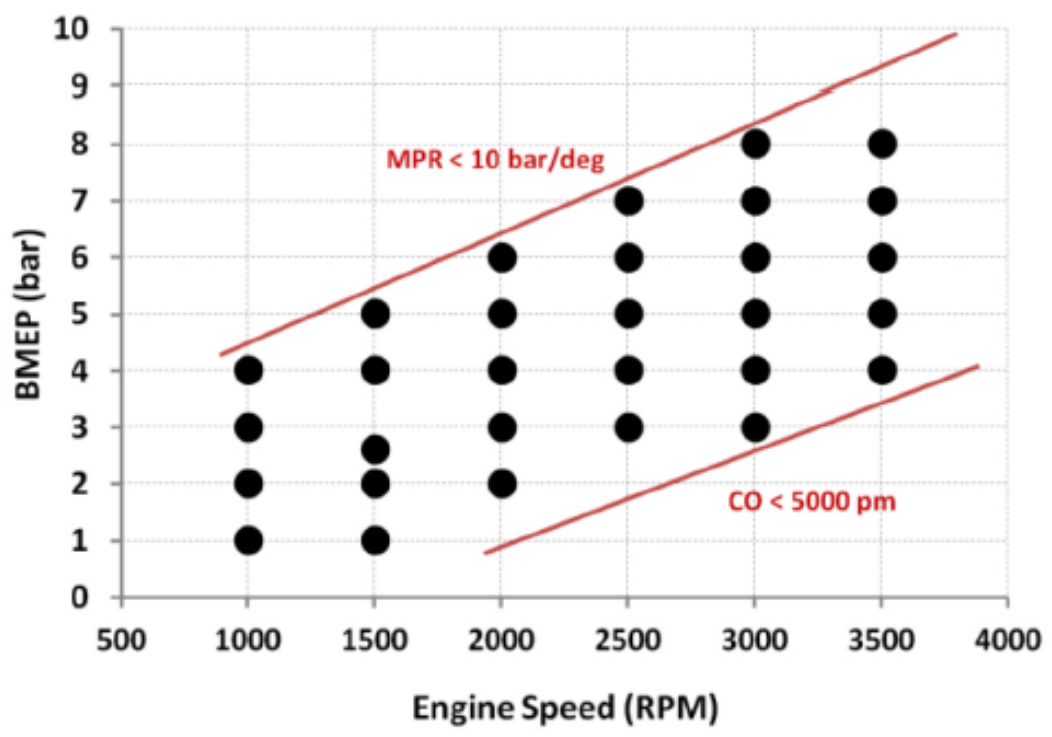

Figure 4: RCCI Operating Points Explored with GM 1.9L CIDI Engine Fueled with Gasoline and ULSD [8]

\subsubsection{Fuel Property Effects on RCCI Combustion}

Literature describing the influence of low and high reactivity fuel properties on $\mathrm{RCCl}$ combustion is limited. The primary low reactivity fuel utilized for $\mathrm{RCCl}$ research has been gasoline, although researchers have experimented with blends of ethanol in gasoline up to 85 percent (E85) [26, 27, 28], nbutanol [29], methanol [7], and natural gas [25]. Hanson, et al. substituted E20 for gasoline in dieselgasoline $\mathrm{RCCl}$ combustion in a light-duty multi-cylinder $\mathrm{CIDI}$ engine. A reduction in PRR and heat release rate (HRR) was observed which allowed for the maximum safe load (dictated by PRR) to be increased from 8 to 10 bar BMEP [26]. Curran, et al. compared RCCl operation with diesel and gasoline to diesel and E85 with the same model engine [27]. Using diesel and E85, a load of 8.8 bar BMEP at 2600 RPM was achievable. RCCl diesel-gasoline approach could not achieve the same load due to excessive incylinder PRRs. The extended load range of diesel-E85 was attributed to the evaporative cooling effect of the ethanol in E85 and higher octane rating ([RON+MON]/2) of E85 (96.7) compared to gasoline (92.1), which created a higher reactivity difference between the high and low reactivity fuels. At lower loads, a higher premixed ratio (percent of low reactivity fuel to total fuel) was necessary to maintain combustion phasing for gasoline versus E85. Dempsey, et al. compared diesel-gasoline versus diesel-methanol RCCI operation noting that the high octane number and charge cooling effect of methanol required an increase in the diesel fraction of total injected fuel [7]. This allowed for an increase of IMEP from approximately 7 bar (gasoline/diesel) to 12 bar (methanol/diesel). Nieman, et al. compared dieselgasoline to diesel-natural gas $\mathrm{RCCl}$ operation using a genetic algorithm coupled with the KIVA3V code at a load of 9 bar IMEP [25]. It was hypothesized and concluded that the larger reactivity difference offered by natural gas (higher octane than gasoline) reduced in-cylinder PRR. The diesel-natural gas case resulted in approximately 2 percent lower gross efficiency compared to diesel-gasoline, which the authors attributed to the higher adiabatic temperature of $\mathrm{CH}_{4}$ in engine like conditions and subsequent greater heat transfer losses. Note that Nieman, et al. states that in the presence of high temperatures, such as engine like conditions, the adiabatic flame temperature of natural gas is higher than gasoline based on the influence of fuel-to-air ratio, which was lower for diesel-gasoline RCCl operation. The 
displacement of air entering the cylinder by injecting natural gas into the intake manifold may have also resulted in lower gross efficiency.

Discussion of high reactivity fuel property effects on $\mathrm{RCCl}$ combustion has been very limited. ULSD has been the primary high reactivity fuel used in $\mathrm{RCCl}$ experiments, but 20 percent biodiesel blended into diesel (B20) [26], gasoline doped with 2-ethylhexyl nitrate (2-EHN) [28, 30], gasoline doped with di-tertbutyl peroxide (DTBP) [31], and cottonseed biodiesel [29] have also been studied. Hanson, et al. compared the combustion and emissions effects of B20-gasoline to diesel-gasoline RCCI [26]. The use of B20 allowed for a "significantly reduced [port fuel injection (PFI)] fraction to maintain similar combustion phasing," which in turn increased the effective $\mathrm{CN}$ of the total fuel mixture. Results demonstrated conflicting trends, where the maximum in-cylinder PRR increased, yet NOx emissions decreased accompanying a reduction in THC emissions, but an increase in $\mathrm{CO}$ emissions. They offer several hypotheses for these phenomena based on boiling point and oxygen content of B20, but no conclusions were made due to limited results.

Hanson, et al. studied the characteristics of gasoline-gasoline doped with 2-EHN (CN improver) versus diesel-gasoline $\mathrm{RCCl}$ on a single-cylinder, heavy-duty $\mathrm{Cl}$ engine [30]. $\mathrm{RCCl}$ with gasoline doped with 3.5 percent by volume 2-EHN resulted in slightly earlier high temperature heat release compared to diesel, although it was hypothesized that this may be attributed to the increased gasoline fuel fraction used (compared to diesel-gasoline $\mathrm{RCCl}$ ) or that the gasoline 2-EHN mixture had more reactivity than the diesel. Low temperature heat release (LTHR) occurred earlier for the gasoline 2-EHN condition, which was attributed to the decomposition of 2-EHN in the direct-injection stream since gasoline does not typically exhibit two stage heat release. The authors noted that each strategy showed similar THC, CO and $\mathrm{NO}_{\mathrm{x}}$ tradeoffs and both strategies were able to meet 2010 federal heavy-duty engine emissions standards for $\mathrm{NO}_{\mathrm{x}}$ emissions at steady state loads of 2 bar and 4.5 bar IMEP at multiple engine speeds.

Ickes, et al. examined the effects of $\mathrm{CN}$ on combustion of diesel-gasoline dual-fuel combustion in a heavy-duty $13 \mathrm{~L}$ six-cylinder engine at multiple steady state speed and load conditions [32]. They used three fuels, a ULSD fuel (44 CN), a low-temperature Fischer-Tropsch fuel (LTFT > 73 CN), and low CN fuel referred to as FACE 1 (30 CN). FACE 1 is one of the nine FACE (fuels for advanced combustion engines) diesel fuels that were designed for parametric studies by the Coordinating Research Council (CRC) and manufactured by Chevron-Phillips Chemical Co [33]. It is important to note that their combustion control approach was more similar to conventional dual-fuel combustion and thus differed from $\mathrm{RCCl}$ in that the diesel injection was initiated near top dead center (TDC), 0 to $20 \mathrm{CA}^{\circ}$ before top dead center (BTDC), and its timing was used to control combustion phasing. Nonetheless, the authors were able to achieve NOx and soot emissions lower than 2010 heavy-duty engine standards (with the same caveat as before about steady state operating conditions) and conclusions were drawn on the effect of $\mathrm{CN}$. Comparable brake thermal efficiency (BTE) was achieved regardless of $\mathrm{CN}$ of the high reactivity fuel with the exception of the lower CN test fuel (FACE 1) at low load conditions, which was presumably related to retarded combustion phasing. Compared to ULSD, the low CN fuel, FACE 1, shifted the operable load range window to a higher load and overall lower soot emissions were observed. The high CN fuel, LTFT, exhibited similar combustion characteristics to the ULSD. Compared to the FACE 1 fuel, the LTFT fuel allowed for "stable operation with high gasoline fractions across a wider load range" [32]. 


\subsection{Fuel Property Effects on LTC}

Studies performed at WVU, and other organizations, have concluded that aromatic content $(\mathrm{AC})$ and distillation temperature of a diesel fuel can affect its advanced combustion behavior in addition to $\mathrm{CN}$ $[14,34,35,36]$. A study performed at WVU (CRC AVFL-16), funded by the CRC to determine fuel property effects on advanced combustion regimes, utilized the FACE diesel fuels. From this study, WVU researchers concluded that CN had the most dominate effect on LTC performance and emissions [14]. This conclusion agreed with results published by researchers at Oak Ridge National Laboratory (ORNL) [35] and Natural Research Council [34] Canada who performed similar research with the FACE diesel fuels. Examining FACE fuels with similar CN number, it was found that fuels with a higher AC produced higher $\mathrm{NO}_{\mathrm{x}}$ emissions [35]. A separate study concluded that higher $\mathrm{AC}$ resulted in a longer ignition delay and increased THC and CO emissions [36]. Fuels with a high AC have also been correlated to increased soot formation and flame temperature compared to fuels with a lower AC for $\operatorname{CDC}[34,35,37]$. Documentation of a direct correlation of higher flame temperatures for high AC fuels with regards to advanced combustion with the FACE diesel fuels was not found. However, the correlation of higher $\mathrm{NO}_{\mathrm{x}}$ emissions from FACE diesel fuels with high AC does suggest that a high AC diesel fuel may produce higher flame temperatures during advanced combustion compared to low AC diesel fuels. With respect to 90 percent distillation temperature (T90), a measure of the back end volatility of a fuel, the researchers from WVU concluded that high CN fuels with a lower T90 produced less soot, although AC may have contributed to this phenomena as well [14]. This phenomenon is demonstrated in Figure 5, where FACE 5 had the highest CN of all fuels tested but a lower T90 and AC than FACE 6 and FACE 8, yet FACE 5 exhibited, on average, lower soot emissions than the other high CN fuels. Note that there are nine data points for each fuel; three "optimal" control configurations (based on pilot and main injection timing and fuel split percentage) with respect to soot and $\mathrm{NO}_{\mathrm{x}}$ emissions and BTE for each specific fuel. The remaining six data points used identical control configurations with the same pilot and main injection timing and fuel split percentage for all fuels. Cho, et al. observed similar results for high CN fuels, demonstrating that fuels with a low T90 produced nearly half the PM emissions as their high T90 counterparts [35].

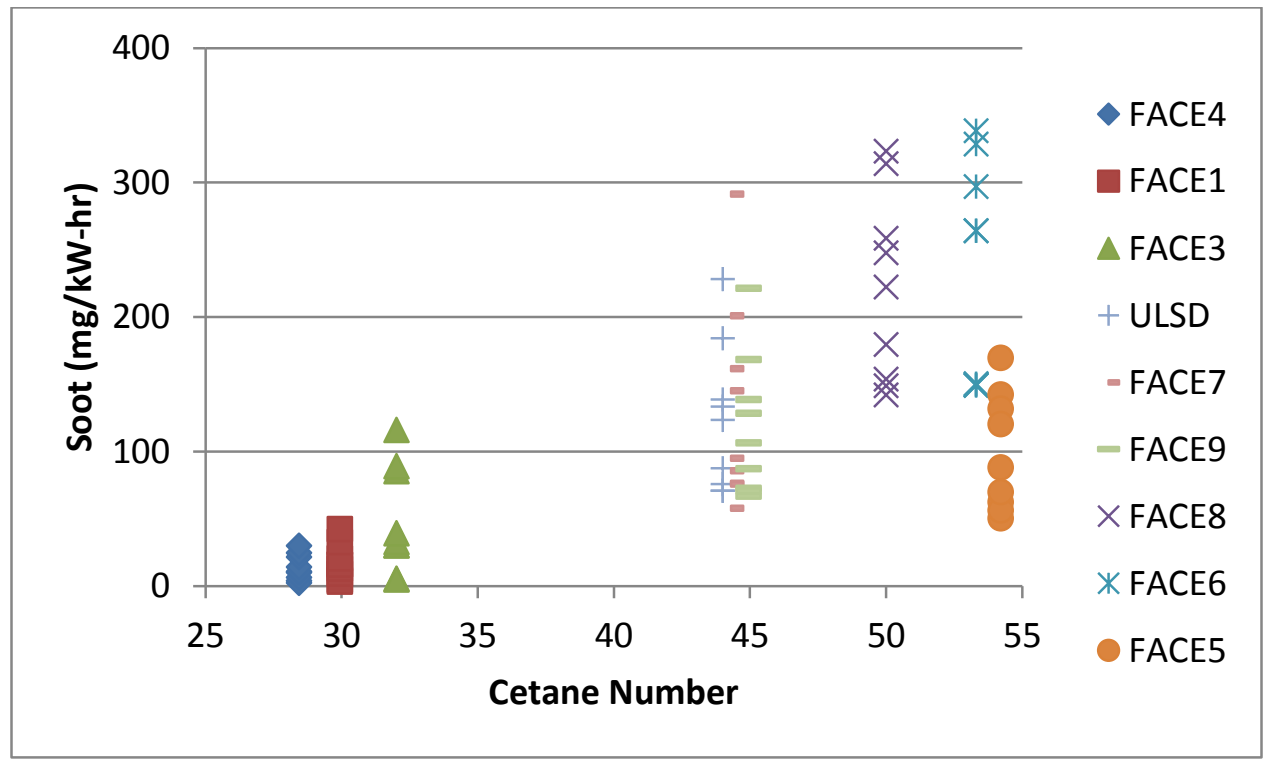

Figure 5: Soot vs. CN from CRC AVFL-16 Project Test Engine Operating in LTC Mode [14] 
At the completion of the CRC AVFL-16 project, WVU retained possession of limited quantities of the FACE diesel fuels and those fuels were used for this current project. Fuel properties for the FACE diesel fuels are detailed further in Section 4.7. 


\section{Hypothesis}

From the surveyed literature, it was apparent that the $\mathrm{CN}$ of the high reactivity fuel had an effect on the combustion characteristics of $\mathrm{RCCl}$, but its role was described as primarily influencing the fraction of low reactivity fuel to high reactivity fuel necessary to control combustion phasing and in-cylinder PRR. Discussion of $A C$ and distillation temperature effects on $\mathrm{RCCl}$ were absent, with the exception of Hanson, et al. theorizing that the higher boiling point and oxygen content of B20 may have contributed to higher PRR, yet lower $\mathrm{NO}_{x}$ and THC emissions compared to diesel-gasoline RCCl. Unfortunately, these hypotheses could not be confirmed because of limited information. Using FACE diesel fuels, researchers from multiple institutions demonstrated that $A C$ and distillation temperature profiles could affect the performance and emissions of similar CN fuels utilized for single fuel LTC strategies. Note that fuels with a similar AC may still have significantly different compositions of paraffin and naphthene hydrocarbon compounds.

Based on the limited documentation of high reactivity fuel property effects on $\mathrm{RCCl}$ and the knowledge of fuel property effects on single fuel LTC, the fundamental question became: How do the key properties of the high reactivity fuels, such as $\mathrm{CN}, \mathrm{AC}$, and T90, affect diesel-natural gas RCCI combustion characteristics (in-cylinder pressure and PRR, HRR and MFB profiles, and combustion efficiency), BTE and FCE, exhaust emissions ( $\mathrm{NO}_{x}, \mathrm{CO}, \mathrm{THC}$, and soot), and the operable load range? Knowledge of these fuel property effects on single fuel LTC obtained from the literature and through the course of the CRC AVFL-16 project, "Fuels to Enable Light-Duty Diesel Advanced Combustion Regimes" provided for the development of the following hypotheses.

- Hypothesis 1 - RCCl with a high CN fuel with a low AC and a low T90 will reduce combustion instabilities and $\mathrm{THC}$ and $\mathrm{CO}$ emissions (compared to other high reactivity fuels) at low load by reducing ignition delay (CN) and promoting vaporization (low T90) while retaining low soot and $\mathrm{NO}_{x}$ emissions (AC). Heavy dilution through EGR and multiple diesel injections per cycle will assist in reducing PRR during combustion of this fuel at high loads.

- Hypothesis 2 - RCCl with a low CN fuel with high AC will extend high load operation (because of increased mixing time due to low $\mathrm{CN}$ ), and reduce combustion instabilities as well as THC and CO emissions (compared to low CN fuels with a low $\mathrm{AC}$ ) at low load operation based on the convention that fuels with higher $\mathrm{AC}$ exhibit higher combustion temperatures.

Exploration of these hypotheses were proposed not only to resolve the effect of high reactivity fuel properties on $\mathrm{RCCl}$, but also to determine which combination of fuel properties provided the largest operating range with lowest combustion instabilities, highest fuel efficiencies, and maximum achievable load with permissible PRR. 


\section{Experimental Setup}

A 2005 GM Z19DTH light-duty Cl engine, the FACE diesel fuels, and locally sourced natural gas were used to investigate the hypotheses. This particular GM Z19DTH was previously used for the CRC AVFL-16 project [14], for which LTC data has been presented in Section 2.4. This engine model has also been utilized by ORNL [8] and UW-Madison for RCCI research [7]. The test engine was installed in a laboratory setup by the author located on the Evansdale campus of WVU as part of the Center for Alternative Fuels, Engines, and Emissions (CAFEE) research facilities.

\subsection{GM Z19DTH Test Engine}

The GM Z19DTH as depicted in Figure 6 is, with regards to technology, a relatively modern 1.9-liter fourcylinder engine typically installed in passenger cars primarily sold in Europe. It features a Bosch common rail DI fuel system, dual overhead camshafts with four valves per cylinder, cooled EGR, and a VGT. The intake manifold is comprised of two intake runners per cylinder, one of which has a variable position swirl valve, which can be used to influence in-cylinder mixing. The other intake runner is unrestricted. An in-cylinder pressure transducer was installed into the combustion chamber of one cylinder via an adapter that replaced the factory glow plug. Crankcase emissions from the engine were routed back into the intake of the engine. Table 2 provides a general summary of the test engine specifications.

Table 2: Test Engine Specifications

\begin{tabular}{|c|c|}
\hline Engine Manufacturer/Model & Model Year 2005 GM Z19DTH \\
\hline Number of Cylinders & 4 \\
\hline Displacement & 1.9 Liters \\
\hline Bore & $82.0 \mathrm{~mm}$ \\
\hline Stroke & $90.4 \mathrm{~mm}$ \\
\hline Compression Ratio & 17.5 \\
\hline Valves Per Cylinder & 4 \\
\hline Fuel Injection System & Bosch High Pressure Common Rail Direct Injection \\
\hline Emissions Technology & EGR, VGT, Swirl Valves \\
\hline
\end{tabular}




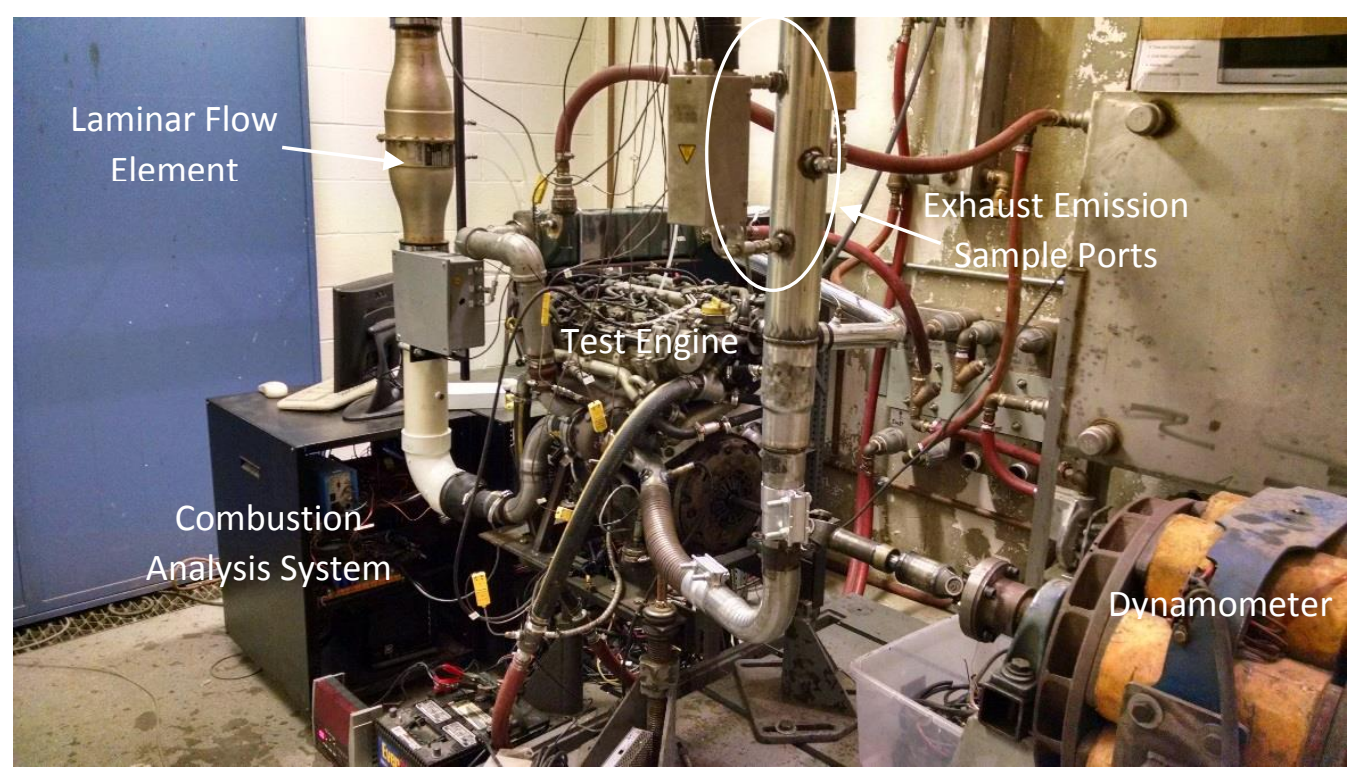

Figure 6: GM Z19DTH Light-Duty CI Engine Installed in the CAFEE Test Cell

Delivery of the low reactivity fuel, which was natural gas for the research presented herein, was accomplished by fuel injectors installed into the intake manifold runners, prior to the intake valves. Due to limited space around the intake manifold runners and the tightly packaged nature of the test engine, A.E.B. S.p.A. model number 238631000 , remote-mounted gaseous fuel injectors were selected. The nozzles for these injectors were installed in the un-restricted intake runner adjacent to the runner containing the swirl valve. The nozzles were then connected to the remote mount injectors via 6 millimeter tubing. Gaseous fuel was supplied to the injectors at approximately 3 bar gauge pressure. Figure 7 and Figure 8 show the installation of the gaseous fuel injection system. This injection into the intake manifold is also referred to as PFI in this document.

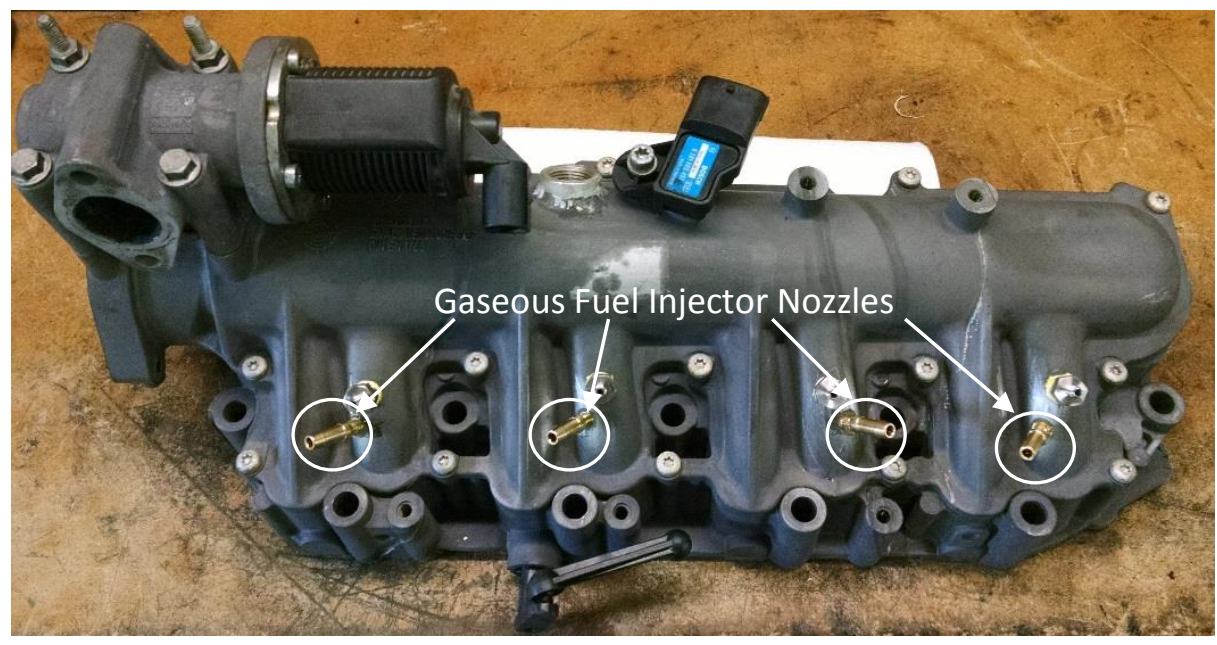

Figure 7: Nozzles for Gaseous Fuel Injectors Installed in the Intake Manifold 


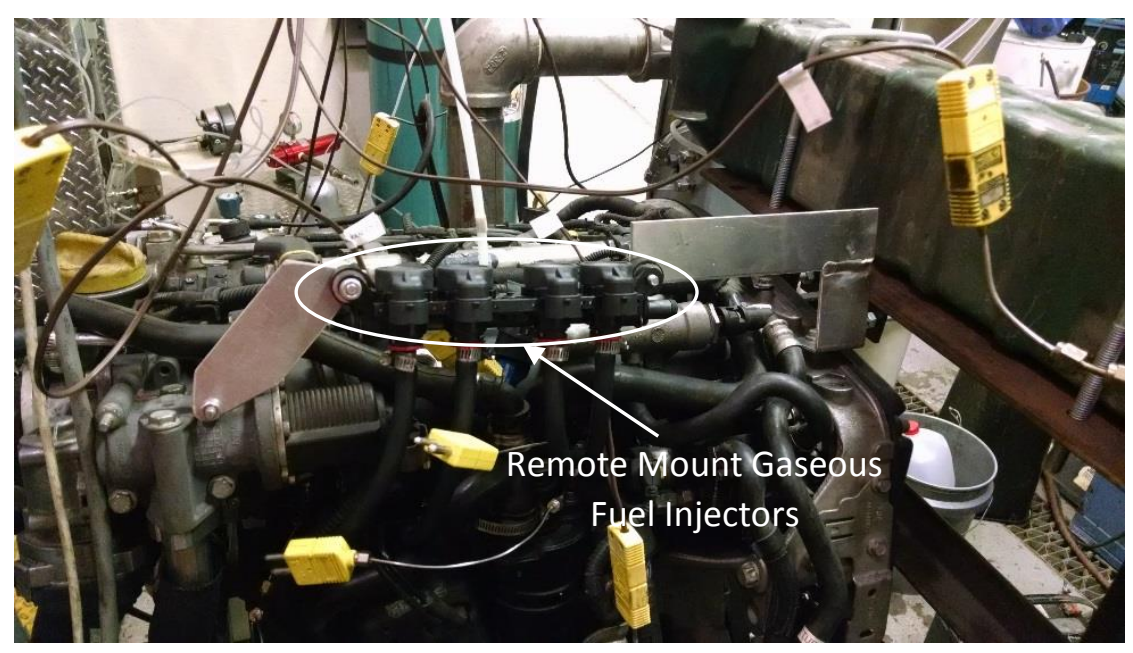

Figure 8: Remote Mount Gaseous Fuel Injectors Installed on the Test Engine

Comprehensive control of the engine and its subsystems was achieved using a Drivven engine controller [38]. At the heart of the Drivven controller was a National Instruments (NI) PXI-1042 series chassis. Drivven developed modules, based on NI CompactRIO technology were then used as the physical drivers for the fuel injectors, EGR valve, swirl valves, VGT actuator and other engine hardware. Additional Drivven modules input sensor feedback from the engine such as temperature and pressures as well as crankshaft and camshaft position signals. NI Lab View software was used in cooperation with a Drivven developed program, Calview, which controlled the engine via the $\mathrm{NI}$ and Drivven hardware and also allowed for control parameter changes in real time.

\subsection{Data Acquisition}

The data acquisition (DAQ) system was comprised of several different hardware and software components. The CAFEE-developed Scimitar software served as the main DAQ and dynamometer control software [39]. It recorded engine and environmental data including temperatures, pressures, and engine performance and emissions measurements. Thermocouples were measured using an ICPCON PET-7019Z DAQ module, while other analog inputs were measured using a Labjack UE-9 Pro.

\subsection{Exhaust Emissions Measurement}

Gaseous exhaust emissions were measured by a modular system of raw sampling systems often referred to as "raw lab" boxes. The system was comprised of a $\mathrm{CO} / \mathrm{CO}_{2}$ analyzer (Figure 9), $\mathrm{THC}$ analyzer (Figure 9), and $\mathrm{NO} / \mathrm{NO}_{x}$ analyzer, each of which contained their own gaseous sampling and DAQ systems. CO and $\mathrm{CO}_{2}$ were measured by a Horiba AIA-220 non-dispersive infrared analyzer (NDIR). THC measurement was provided by a Horiba FIA-236 flame ionization detector (FID). NO and $\mathrm{NO}_{x}$ were quantified using an ECO PHYSICS CLD 822 CM h chemiluminescence analyzer. Exhaust oxygen content was measured by a Rosemount Analytical 755R paramagnetic oxygen analyzer. Soot emissions were characterized by an AVL 483 micro soot sensor, which relied on a photo acoustic based measurement principle. 


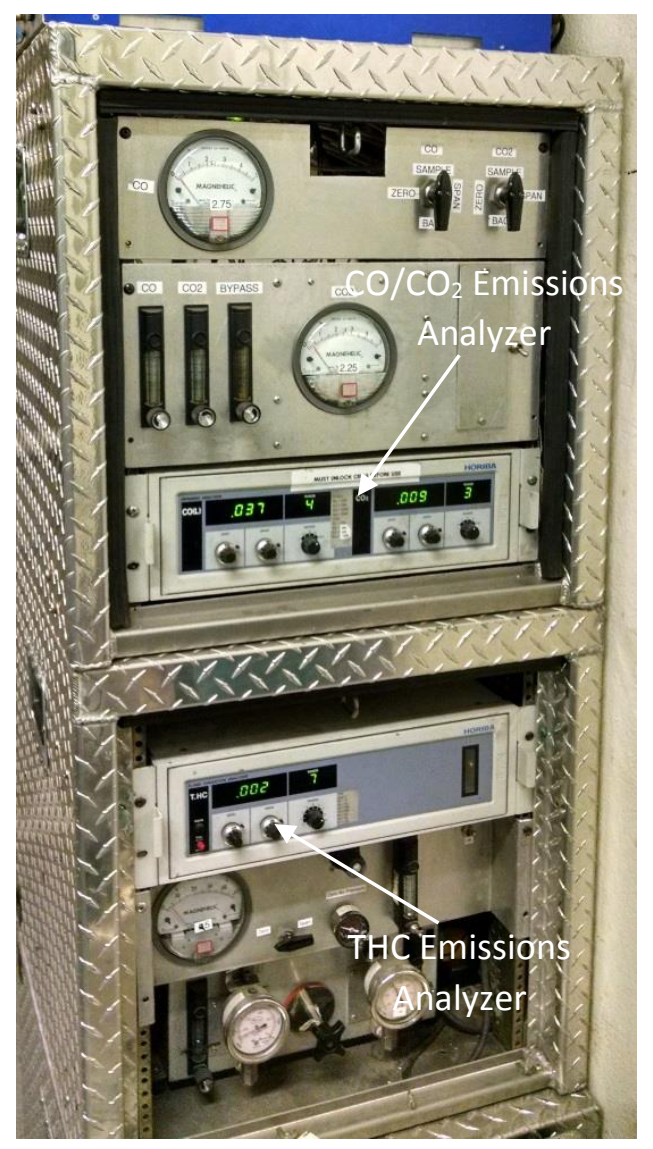

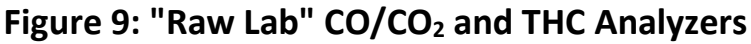

\subsection{Intake Air and Fuel Flow Measurement}

Engine intake airflow was measured using a 4-inch Meriam laminar flow element (LFE) model 50MC2-4. Utilizing an OMEGADYNE PX209-30V15G5V absolute pressure transducer, OMEGA PR-14-2-100-3/16-9-E resistance temperature detector (RTD), and OMEGA HX52 humidity transducer, mass rate of the intake air was calculated from the LFE Ashcroft IXLdp differential pressure (DP) measurement. Diesel fuel consumption was measured gravimetrically by a Brecknell MBS-6000 scale. Gaseous fuel consumption was measured using a Sierra C100H-2-NR-16-OV1-SV1-PV2-V1-S1-C0 mass flow controller (MFC). The control valve for the MFC was set fully open, and the gaseous fuel injectors installed on the engine's intake manifold were used to modulate gaseous fuel flow.

\subsection{Dynamometer and Control}

A Mustang eddy current dynamometer, model A 400, rated at 400 horsepower (HP) absorbing capability was used to load the test engine. A Bardac 3200i isolated DC drive was used to power the eddy current dynamometer while a Bardac Drive.Web Smarty was used to control the 3200i drive. The Smarty communicated with Scimitar, allowing the operator to control engine loading via the dynamometer and also adjust a proportional integral derivative (PID) controller programmed to hold the engine at a constant speed. 


\subsection{In-Cylinder Pressure and Combustion Analysis}

Combustion analysis was performed on data collected from an in-cylinder pressure transducer and crank angle position measurements. The in-cylinder pressure transducer was a Kistler 6058A piezoelectric pressure transducer installed into the cylinder's glow plug port with a Kistler 6544Q adapter. A Kistler 5010B charge amplifier was used to condition the signal. A BEI express XH25D-SS-720-ABZC-28V/VSM18 optical encoder was used to determine engine crank angle position and calculate cylinder volume for the pressure analysis. In-cylinder pressure and crank angle position signals were recorded via a $\mathrm{NI}$ SCB-68 DAQ. In house combustion data collection and analysis software was used. From the in-cylinder pressure and crank angle position, several other combustion parameters were calculated by the analysis software including IMEP, PRR, gross HRR, MFB, and combustion duration. IMEP was defined as the indicated work per engine cycle divided by the cylinder volume displaced per cycle [11]. The indicated work per cycle for a given cylinder was calculated by integrating the pressure over the change in volume of the cylinder [40]. PRR was determined by taking the derivative of the in-cylinder pressure with respect to the crank angle. Gross HRR was derived using the first law of thermodynamics assuming a uniform pressure, uniform temperature and ideal gas [11]. MFB was also found using the first law of thermodynamics and was calculated by dividing the cumulative gross heat released with respect to crank angle by the total heat released [41]. Combustion duration, for this study, was calculated by subtracting the crank angle location of 5 percent MFB (CA5) from the crank angle location of 95 percent MFB (CA95).

\subsection{Fuels}

The low reactivity fuel that was injected into the intake manifold runner was CNG that was sourced from a local vehicle refueling station. A bulk CNG tank for heavy-duty vehicle applications was filled three times over the course of the study. Samples were obtained from each batch of CNG. The analysis from each of these samples, average values, standard deviations, and coefficients of variation (COV) are presented in Table 3. 
Table 3: CNG Fuel Properties

\begin{tabular}{|c|c|c|c|c|c|c|}
\hline Sample Date & $\mathbf{1 1 / 1 7 / 2 0 1 5}$ & $\mathbf{1 2 / 8 / 2 0 1 5}$ & $\mathbf{1 / 2 7 / 2 0 1 6}$ & Average & $\begin{array}{c}\text { Standard } \\
\text { Deviation }\end{array}$ & CoV \\
\hline Methane (Mol \%) & 95.1 & 95.0 & 95.0 & 95.0 & 0.0621 & $0.0653 \%$ \\
\hline Ethane (Mol \%) & 3.18 & 3.19 & 3.28 & 3.22 & 0.0463 & $1.44 \%$ \\
\hline Propane (Mol \%) & 0.591 & 0.536 & 0.616 & 0.581 & 0.0333 & $5.73 \%$ \\
\hline Butane (Mol \%) & 0.231 & 0.203 & 0.239 & 0.224 & 0.0152 & $6.76 \%$ \\
\hline Pentane+ (Mol \%) & 0.153 & 0.187 & 0.154 & 0.165 & 0.0155 & $9.42 \%$ \\
\hline Nitrogen (Mol \%) & 0.458 & 0.556 & 0.484 & 0.499 & 0.0415 & $8.31 \%$ \\
\hline Oxygen (Mol \%) & 0.00770 & 0.0258 & 0.00940 & 0.0143 & 0.00816 & $57.1 \%$ \\
\hline $\begin{array}{c}\text { Carbon Dioxide } \\
\text { (Mol \%) }\end{array}$ & 0.271 & 0.266 & 0.262 & 0.266 & 0.00351 & $1.32 \%$ \\
\hline $\begin{array}{c}\text { Gross Heating Value } \\
\text { (BTU/SCF Saturated) }\end{array}$ & 1033 & 1032 & 1034 & 1033 & 0.902 & $0.0873 \%$ \\
\hline $\begin{array}{c}\text { Specific Gravity* } \\
\text { Molecular Weight } \\
\text { (Ib/lbmol) }\end{array}$ & 0.588 & 0.588 & 0.588 & 0.588 & 0.000327 & $0.0556 \%$ \\
\hline
\end{tabular}

*With respect to air

Fuel property analyses presented in Table 3 were performed on the three different batches of CNG to determine if there were any significant differences in the composition of the CNG used throughout the high reactivity fuel property effects study. Significant changes in the CNG composition could bias the perceived high reactivity fuel property effects. The COV of each constituent of the CNG quantifies the variation between the batches. However, it is important to consider the molar percentage in addition to the COV. For example, COV for the oxygen content of the three different batches was very high at $57.1 \%$, but this was rather insignificant because of its miniscule molar percentage in the CNG. Since the $\mathrm{COV}$ of $\mathrm{CH}_{4}$ was less than one tenth of one percent and it comprised the largest portion of the $\mathrm{CNG}$, it was concluded that the three different batches of CNG would have no significant effect on high reactivity fuel property trends. The very low COVs for gross heating value and specific gravity (SG) further support this conclusion.

The CRC FACE diesel fuels and a certification-grade ULSD were used as the high reactivity fuels. These fuels comprised a matrix of nine diesel fuels with varying $C N, A C$, and T90 developed to explore fuel property effects on advanced combustion regimes. A visualization of the FACE diesel fuel properties as designed is provided in Figure 10. However, the actual fuel properties differed from the design specification and thus the measured properties of the fuels are presented in Table 4. Additionally, hydrogen-to-carbon ratio and the net heat of combustion of the Cert fuel was not available. A typical diesel fuel value of 1.8 for hydrogen-to-carbon ratio was used for chemical balance calculations for the Cert fuel. A range of typical net heat of combustion values for light diesel (18570 BTU/lb) and heavy diesel (18000 BTU/lb) were investigated to calculate efficiency values for the Cert fuel. The average percent difference in FCE during $\mathrm{RCCl}$ operation with the Cert fuel when using the net heat of combustion values for light diesel versus heavy diesel was 0.87 percent. This percent difference was 
considered miniscule, and based on the low AC of the Cert fuel, $18570 \mathrm{BTU} / \mathrm{lb}$ was selected for final calculations.

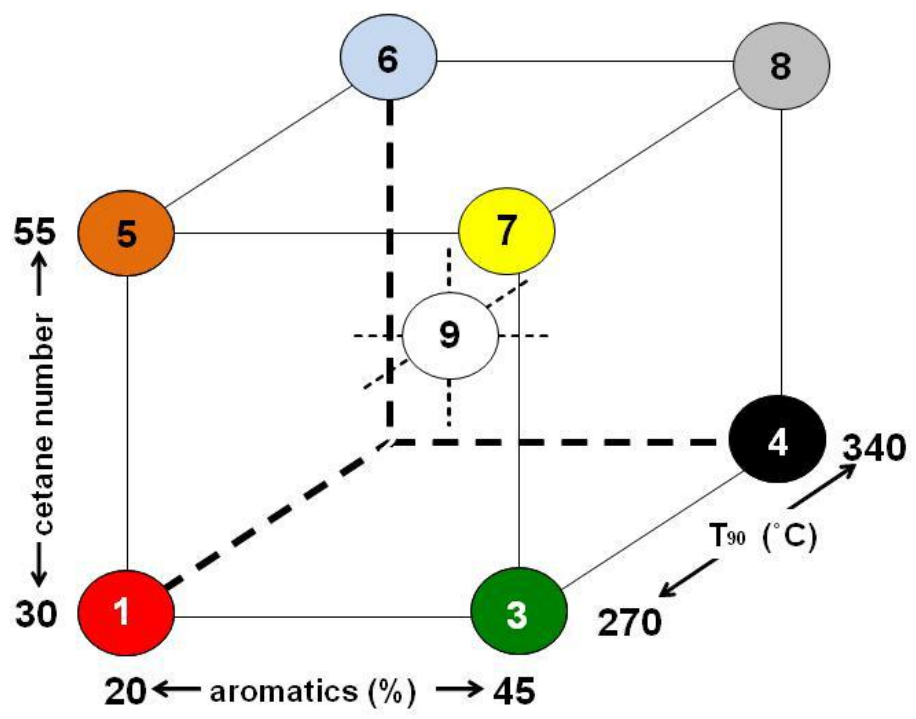

Figure 10: Target Design Properties of the FACE Diesel Fuels Used in This Study [14]

Table 4: FACE and Cert Diesel Fuel Properties [33]

\begin{tabular}{|c|c|c|c|c|c|c|c|c|c|}
\hline Property & FACE 4 & FACE 1 & FACE 3 & FACE 7 & FACE 9 & Cert & FACE 8 & FACE 6 & FACE 5 \\
\hline Cetane Number & 28.4 & 29.9 & 32.0 & 44.3 & 45.0 & 45.6 & 50.0 & 53.3 & 54.2 \\
\hline $\begin{array}{c}\text { Aromatic } \\
\text { Content } \\
\text { (Mass \%) }\end{array}$ & 40.7 & 26.1 & 50.0 & 46.2 & 37.0 & 10.4 & 43.5 & 21.1 & 22.2 \\
\hline $\begin{array}{c}\text { 90\% Distillation } \\
\text { Temperature ('C) }\end{array}$ & 337 & 269 & 270 & 267 & 321 & 308 & 342 & 341 & 276 \\
\hline $\begin{array}{c}10 \% \text { Distillation } \\
\text { Temperature ('C) }\end{array}$ & 177 & 157 & 172 & 197 & 189 & 224 & 247 & 227 & 187 \\
\hline Specific Gravity* & 0.836 & 0.808 & 0.840 & 0.838 & 0.847 & 0.847 & 0.868 & 0.841 & 0.809 \\
\hline $\begin{array}{c}\text { Hydrogen-to- } \\
\text { Carbon Ratio }\end{array}$ & 1.82 & 1.96 & 1.75 & 1.77 & 1.79 & - & 1.70 & 1.87 & 1.97 \\
\hline $\begin{array}{c}\text { Net Heating } \\
\text { Value (MJ/kg) }\end{array}$ & 42.5 & 42.8 & 42.1 & 42.4 & 42.5 & - & 42.2 & 42.8 & 42.9 \\
\hline
\end{tabular}

*With respect to water

The fuel properties and any potential interactions among them were investigated. Each fuel property was plotted against another fuel property and a linear fit was applied. Instances where the $R^{2}$ value of the linear fit was greater than 0.5 are displayed in Table 5. 
Table 5: Fuel Property Interactions with $\mathrm{R}^{2}$ Greater than 0.5

\begin{tabular}{|c|c|}
\hline Fuel Property Interaction & $\mathbf{R}^{\mathbf{2}}$ from Linear Fit \\
\hline Cetane Number vs. 10\% Distillation Temperature & 0.53 \\
\hline Aromatic Content vs. Net Heat of Combustion & 0.95 \\
\hline $10 \%$ Distillation Temperature vs. Specific Gravity & 0.54 \\
\hline Specific Gravity vs. Hydrogen-to-Carbon Ratio & 0.82 \\
\hline
\end{tabular}

Among the interactions presented in Table 5, the interaction between $\mathrm{CN}$ and 10 percent distillation temperature (T10) is of interest based on the presence of both properties in trends of the data presented in Chapter 6 . The trend line in Figure 11 suggests that T10 increases as CN increases. The T10 of the low CN fuels are unanimously lower than the T10 of the medium and high CN fuels. However, progressing to the medium and high $\mathrm{CN}$ fuel groupings, this correlation dissipates, noting that the highest CN fuel, FACE 5 has the lowest T10 value with the exception of the low CN fuels. When correlating high reactivity fuel properties to characteristics of $\mathrm{RCCl}$, a $\mathrm{T} 10$ correlation may also be present when a CN correlation is observed, but examining the T10 effect for medium and high CN fuels will help to reveal whether this correlation is due to $\mathrm{CN}$ or T10 itself.

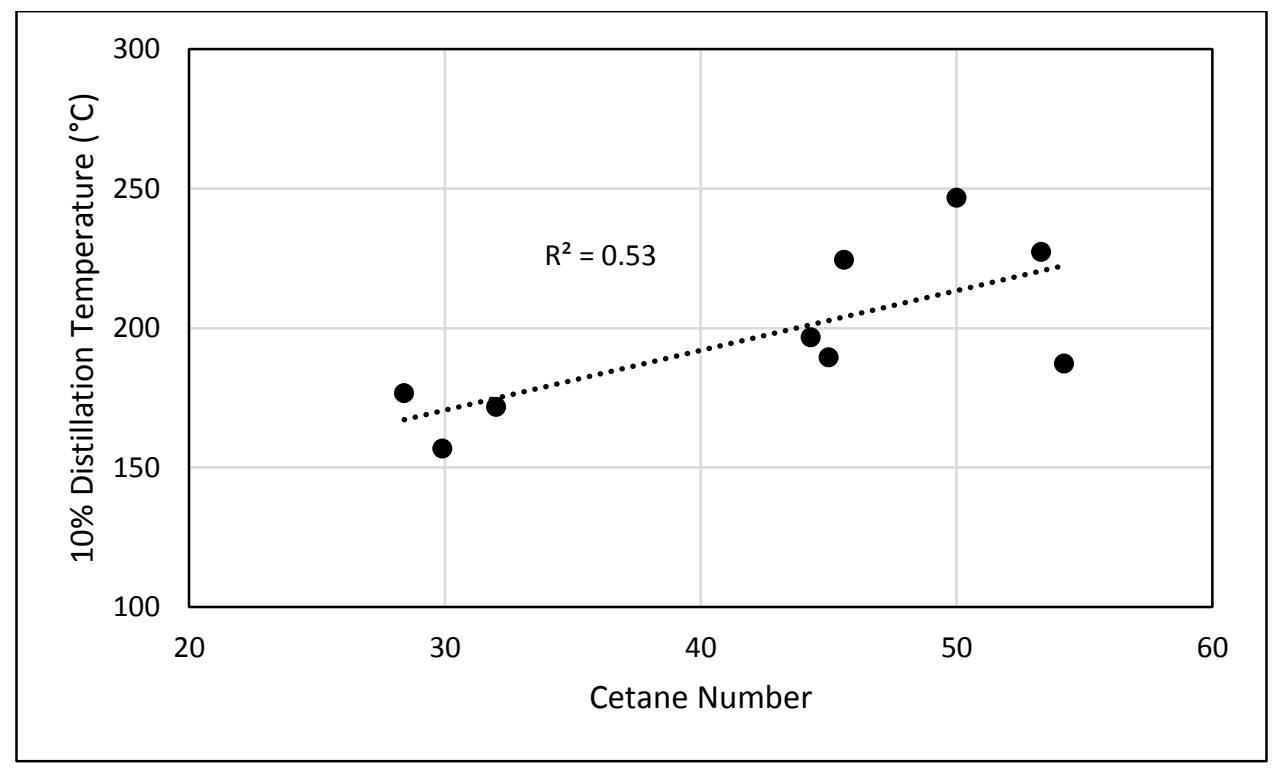

Figure 11: T10 versus CN for the FACE and Cert Diesel Fuels

\subsection{Data Reduction}

A Matlab routine was written to reduce data and calculate emissions and performance parameters in an automated manner. Since emissions measurements were taken from the raw exhaust and no direct exhaust flow measurement was available, the measured intake air mass flow and fuel mass flow were summed to obtain exhaust flow. Combined with the density of a particular emissions constituent, a mass rate of the emissions constituent could be determined using this exhaust flow rate. To provide a level of confidence in this exhaust flow calculation, the measured air-to-fuel ratio was compared to the air-to-fuel ratio calculated by two chemical balance approaches using exhaust emissions data detailed by Heywood [11]. An upper threshold of 10 percent difference between these two values was selected to ensure confidence in the emissions and flow measurements for an individual test point. Ideally, the 
difference would be less than five percent, but given the increased uncertainties presented by operation on multiple fuels, their properties and measurements, the number of exhaust analyzers used, as well as the departure from standard combustion to $\mathrm{RCCl}, 10$ percent was deemed reasonable.

\subsubsection{Intake Air Flow Calculations}

Intake airflow was calculated primarily using a LFE detailed in Section 4.4. A mass airflow sensor used by the Drivven engine controller offered a second measurement to compare to the LFE measurement. The DP across the LFE was used to calculate an actual volumetric flow according to Equation 1 from the LFE manufacturer [42]. To obtain a standard volumetric flow rate of dry air at the LFE manufacturer's standard conditions, $21.1{ }^{\circ} \mathrm{C}(294.25 \mathrm{~K})$ and $101.3 \mathrm{kPa}$ absolute pressure, this actual volumetric flow was then corrected for ambient temperature, ambient pressure, the viscosity of moist air, and the density of moist air as displayed in Equation 2.

\section{Equation 1: Initial Volumetric Flowrate of Intake Air from LFE [42]}

$$
\dot{Q}_{\text {Intake Air Initial }}=B * D P+C * D P^{2}
$$

Where DP is the differential pressure measured across the LFE, and $B$ and $C$ are constant coefficients provided by the LFE manufacturer. For the four inch LFE used during this project, $B$ and $C$ were equal to 49.3839 and -0.139778 , respectively. The standard flow is then:

\section{Equation 2: Standard Volumetric Flow Rate of intake Air Calculation from LFE [42]}

$$
\dot{Q}_{\text {Intake Air STD }}=\dot{Q}_{\text {Intake Air Initial }} *\left(\frac{\mu_{\text {STD }}}{\mu_{\text {Moist Air }}}\right) *\left(\frac{T_{S T D}}{T_{\text {Flow }}}\right) *\left(\frac{P_{\text {Flow }}}{P_{S T D}}\right) *\left(\frac{\rho_{\text {Moist Air }}}{\rho_{\text {Dry Air }}}\right)
$$

Where $\mu_{\text {STD }}$ is the dynamic viscosity of dry air at the standard temperature and pressure. $T_{\text {STD }}$ was 294.25 K. TFlow is the temperature of the air flowing through the LFE. PSTD was 101.3 kPa. P Plow is the absolute pressure of the air flowing through the LFE, and $\rho_{\text {Dry Air }}$ is the density of dry air at the standard temperature and pressure. Dynamic viscosity of the moist air, $\mu_{\text {Moist Air }}$ is calculated using Equation 3.

\section{Equation 3: Dynamic Viscosity of Moist Air [43]}

$$
\mu_{\text {Moist Air }}=\left(\frac{\mu_{A}}{1+\Phi_{A V} * x_{m}}\right)+\left(\frac{\mu_{V}}{1+\frac{\Phi_{V A}}{x_{m}}}\right)
$$

Where the viscosity of dry air, $\mu_{\mathrm{A}}$ is calculated using Equation 4.

\section{Equation 4: Dynamic Viscosity of Dry Air [43]}

$$
\mu_{\mathrm{A}} * 10^{6}[\mathrm{~Pa} * \mathrm{~s}]=0.40401+0.074582 * \mathrm{~T}-5.7171 * 10^{-5 *} \mathrm{~T}^{2}+2.9928 * 10^{-8 *} \mathrm{~T}^{3}-6.2524 * 10^{-12 *} \mathrm{~T}^{4}
$$

Where $\mathrm{T}$ is the temperature in Kelvin (K) of air flowing through the LFE. The dynamic viscosity of the water vapor in air, $\mu_{v}$ is calculated using Equation 5.

Equation 5: Dynamic Viscosity of Water Vapor in Moist Air [43]

$$
\mu_{V} * 10^{6}[\mathrm{~Pa} * s]=\frac{\sqrt{\frac{T}{647.27}}}{0.0181583+0.0177624 *\left(\frac{647.27}{T}\right)+0.0105287 *\left(\frac{647.27}{T}\right)^{2}-0.0036744 *\left(\frac{647.27}{T}\right)^{3}}
$$


$\Phi_{\mathrm{AV}}$ and $\Phi_{\mathrm{VA}}$ are calculated using Equation 6 and Equation 7, respectively.

Equation 6: $\Phi_{A v}$ Calculation [43]

$$
\Phi_{A V}=\frac{\left[1+\left(\frac{\mu_{A}}{\mu_{V}}\right)^{0.5} *\left(\frac{m_{V}}{m_{A}}\right)^{0.25}\right]^{2}}{2 \sqrt{2} *\left(1+\frac{m_{A}}{m_{V}}\right)^{0.5}}
$$

Equation 7: $\Phi_{\mathrm{VA}}$ Calculation [43]

$$
\Phi_{V A}=\frac{\left[1+\left(\frac{\mu_{V}}{\mu_{A}}\right)^{0.5} *\left(\frac{m_{A}}{m_{V}}\right)^{0.25}\right]^{2}}{2 \sqrt{2} *\left(1+\frac{m_{V}}{m_{A}}\right)^{0.5}}
$$

Where $m_{A}$ and $m_{v}$ are the molecular masses of dry air and water vapor, respectively. $X_{m}$ is calculated using Equation 8.

\section{Equation 8: $x_{m}$ Calculation [43]}

$$
x_{m}=1.61 * x
$$

Where $\mathrm{x}$ is the absolute humidity calculated using Equation 9.

Equation 9: Absolute Humidity [40]

$$
x=0.622 * \frac{P_{V}}{P-P_{V}}
$$

Where $\mathrm{P}$ is the ambient absolute air pressure and $\mathrm{P}_{\mathrm{v}}$ is the vapor pressure calculated using Equation 10.

\section{Equation 10: Vapor Pressure [40]}

$$
P_{V}=R H * P_{S a t}
$$

Where $\mathrm{RH}$ is the measured relative humidity and $\mathrm{P}_{\mathrm{Sat}}$ is the saturation pressure at the intake air temperature. This can be found in lookup tables or calculated using Equation 11 from the Code of Federal Regulations (CFR) Title 40 Part 1065 Subpart G.

Equation 11: Saturation Pressure Equation 1065.645-1 from CFR Title 40 Part 1065 Subpart G [44]

$$
\begin{aligned}
\log _{10} P_{\text {Sat }}[k P a] & =10.79574 *\left(1-\frac{273.16}{T}\right)-5.028 \\
& * \log _{10}\left(\frac{T}{273.16}\right)+1.50475 * 10^{-4} *\left(1-10^{-8.2969 *\left(\frac{T}{273.16}-1\right)}\right)+0.42873 * 10^{-3} \\
& *\left(10^{4.76955 *\left(1-\frac{273.16}{T}\right)}-1\right)-0.2138602
\end{aligned}
$$

The density of the moist air, $\rho_{\text {Moist Air, }}$ is calculated using Equation 12. 


\section{Equation 12: Density of Moist Air}

$$
\rho_{\text {Moist Air }}\left[\frac{m^{3}}{k g}\right]=\frac{1+x}{461.56 *(0.62198+x)} * \frac{P}{T}
$$

Where $\mathrm{x}$ is the ambient absolute humidity (Equation 9), $\mathrm{P}$ is the ambient absolute pressure in Pascals, and $T$ the ambient temperature in Kelvin. The standard volumetric flowrate (Equation 2) of intake air can then be used to calculate the mass flow rate of intake air using the standard density of dry air at $294.25 \mathrm{~K}$ and $101.3 \mathrm{kPa}$ with Equation 13.

\section{Equation 13: Mass Flowrate of Intake Air}

$$
\dot{m}_{\text {Intake Air }}=\dot{Q}_{\text {Intake Air STD }} * \rho_{\text {STD Dry Air }}
$$

\subsubsection{Fuel Flow rate Calculations}

High reactivity fuel flow rate (diesel fuel) was calculated using a gravimetric scale. Since all engine tests were performed at a steady state engine speed and load, a linear fit was applied to the fuel scale mass data as a function of time using the Matlab command polyfit in first order fashion. Polyfit uses a least squares approach to fit an equation to a data array. The slope of the fitted equation was the high reactivity fuel mass flowrate. The CNG fuel mass flowrate was reported directly from the MFC.

\subsubsection{Chemical Balances of Exhaust Emissions to Determine Air-to-Fuel Ratio}

A chemical balance, which balances oxygen and utilizes dry or dry corrected emissions measurements of $\mathrm{CO}, \mathrm{CO}_{2}, \mathrm{NO}, \mathrm{NO}_{2}$, and $\mathrm{THC}$, and a chemical balance based on wet THC, $\mathrm{NO}$, and $\mathrm{NO}_{2}$ and dry $\mathrm{CO}, \mathrm{CO}_{2}$ and $\mathrm{O}_{2}$ were used to determine air-to-fuel ratio and equivalence ratio, respectively, from exhaust emissions measurements. As noted previously, this calculated air-to-fuel ratio or equivalence ratio was compared to the air-to-fuel ratio derived from direct measurements to ensure quality of the emissions data. In order to perform either chemical balance, select properties of the fuel must be known. For an engine operating on a single fuel this is trivial, but when operating on two fuels, equivalent values must be calculated for these properties. The oxygen balance requires molecular mass and hydrogen-tocarbon ratio of the fuel while the chemical balance based on wet THC and dry inorganic gas requires stoichiometric air-to-fuel ratio, number of moles of carbon in the fuel and the number of moles of hydrogen in the fuel [11]. Both approaches require an equilibrium constant, $\mathrm{K}$ not to be confused with Kelvin, which is also denoted by $\mathrm{K}$, to determine the amount of water produced by the combustion process. A value of 3.5 was chosen based on Heywood which notes that "for lean mixtures, varying the value of $\mathrm{K}$ between 1.5 and 5.5 had a negligible effect" on the computed air-to-fuel ratio [11].

For the chemical balance calculations, it was assumed that the diesel fuel (high reactivity fuel) contained 16 moles of carbon, no moles of oxygen, and the moles of hydrogen were determined by Equation 14.

\section{Equation 14: Number of Moles of Hydrogen in Diesel Fuel}

$$
n_{\text {H,Diesel }}=y_{\text {Diesel }} * n_{C, \text { Diesel }}
$$

Where $\mathrm{n}_{H, \text { Diesel }}$ is the number of moles of hydrogen in the diesel fuel, YDiesel is the hydrogen-to-carbon ratio of the diesel fuel, and $\mathrm{n}_{c, \text { Diesel }}$ is number of moles of carbon in the diesel fuel. An average from the fuel analyses of the CNG used as the low reactivity fuel measured 1.05 moles of carbon, $\mathrm{n}_{\mathrm{C}, \mathrm{CNG}}$, and 4.08 
moles of hydrogen, $\mathrm{n}_{\mathrm{H}, \mathrm{CNG}}$ in the fuel. This equated to a hydrogen-to-carbon ratio of 3.88. Equation 15 was used to find the individual molecular mass of both fuels.

Equation 15: Molecular Mass of Diesel, CNG, or Mixture

$$
M_{\text {Fuel }}=12.011 * n_{C, \text { Fuel }}+1.008 * n_{H, \text { Fuel }}
$$

The molar fuel flowrates for diesel and CNG were then calculated by dividing the fuel mass flow rate by the molecular mass. The molar flowrate of each fuel was then used in Equation 16 and Equation 17 to determine the equivalent number of hydrogen and carbon moles in the fuel mixture.

Equation 16: Number of Moles of Hydrogen in the Fuel Mixture

$$
n_{H, \text { Fuel Mix }}=\frac{n_{H, C N G} * \dot{n}_{C N G}+n_{H, \text { Diesel }} * \dot{n}_{\text {Diesel }}}{\dot{n}_{C N G}+\dot{n}_{\text {Diesel }}}
$$

Equation 17: Number of Moles of Carbon in the Fuel Mixture

$$
n_{C, \text { Fuel Mix }}=\frac{n_{C, C N G} * \dot{n}_{C N G}+n_{C, \text { Diesel }} * \dot{n}_{\text {Diesel }}}{\dot{n}_{C N G}+\dot{n}_{\text {Diesel }}}
$$

Where nं signifies molar flowrate. These values were then used to determine the equivalent hydrogento-carbon ratio and equivalent molecular mass of the fuel mixture in the same approach used in Equation 15. The stoichiometric air-to-fuel ratio was found by a chemical balance; Equation 18 displays a simplified form.

Equation 18: Stoichiometric Air-to-Fuel Ratio for the Fuel Mixture

$$
\bar{F}_{\text {Stoich }}=\frac{M_{\text {Air }} *\left(n_{C, \text { Fuel Mix }}+\frac{n_{H, \text { Fuel Mix }}}{4}\right) * 4.76}{M_{\text {Fuel Mix }}}
$$

Where the constant 4.76 is a factor of the idealized moles of air containing 21 percent $\mathrm{O}_{2}$ and 79 percent $\mathrm{N}_{2}$. Equation 19 details the calculations to determine air-to-fuel ratio by balancing oxygen [11]. The equation requires each component to be measured with the same moisture content. Thus, emissions constituents that were measured wet (i.e. without a mechanism to remove water) were converted to dry measurements and emissions constituents measured after sample dryer were converted to fully dry for use in Equation 19.

\section{Equation 19: Air-to-Fuel Ratio Determined by Oxygen Balance Approach [11]}

$$
\frac{A}{F}=4.773 *\left(\frac{M_{\text {Air }}}{M_{\text {Fuel Mix }}}\right) \frac{\left[\mathrm{CO}_{2}\right]+\left(\frac{[\mathrm{CO}]}{2}\right)+\left(\frac{\left[\mathrm{H}_{2} \mathrm{O}\right]}{2}\right)+\left(\frac{[\mathrm{NO}]}{2}\right)+\left[\mathrm{NO}_{2}\right]+\left[\mathrm{O}_{2}\right]}{[\mathrm{THC}]+[\mathrm{CO}]+\left[\mathrm{CO}_{2}\right]}
$$

Where all of the emissions constituents are in molar concentration (designated by [ ]). To determine $\mathrm{NO}_{2}$ for use in Equation 19, an assumption was made that the $\mathrm{NO}_{\mathrm{x}}$ emissions were comprised wholly of $\mathrm{NO}$ and $\mathrm{NO}_{2}$. Thus, the molar concentration of $\mathrm{NO}_{2}$ was the measured molar concentration of $\mathrm{NO}$ subtracted from the measured molar concentration of $\mathrm{NO}_{x}$. The molar concentration of $\mathrm{H}_{2} \mathrm{O}$ in the exhaust used in Equation 19 was found using Equation 20. 


\section{Equation 20: Exhaust Molar $\mathrm{H}_{2} \mathrm{O}$ Concentration [11]}

$$
\left[\mathrm{H}_{2} \mathrm{O}\right]=.5 * y_{\text {Fuel Mix }} * \frac{\left[\mathrm{CO}_{2}\right]+[\mathrm{CO}]}{\left[[\mathrm{CO}] /\left(\mathrm{K} *\left[\mathrm{CO}_{2}\right]\right)\right]+1}
$$

$\mathrm{CO}, \mathrm{CO}_{2}$, and $\mathrm{O}_{2}$ were measured after the sample had passed through a Peltier chiller. This only removed a portion of the water in the sample, not the full amount. Equation 21 was used to correct these measurements to an absolutely dry measurement.

Equation 21: "Dry" to Absolute Dry Correction Equation 1065.655-14 from CFR Title 40 Part 1065 Subpart G for Emissions Measurements with Sample Dryer [44]

$$
[\text { Emission }]_{\text {Dry }}=\frac{[\text { Emission }]_{\text {Meas }}}{1-\left[\mathrm{H}_{2} \mathrm{O}\right]_{[\text {Emission }]}}
$$

Where [Emission] represents the molar concentration of the emission constituent being measured (e.g. CO), and $\left[\mathrm{H}_{2} \mathrm{O}\right]_{[E \text { Emission] }}$ is the molar concentration of $\mathrm{H}_{2} \mathrm{O}$ remaining in the sample after the sample dryer which was determined using Equation 22.

Equation 22: Molar Concentration of Water Vapor in Emissions Sample after Sample Dryer Derived from Equation 1065.645-3 from CFR Title 40 Part 1065 Subpart G [44]

$$
\left[\mathrm{H}_{2} \mathrm{O}\right]_{[\text {Emission }]}=\frac{P_{\text {Dryer }}}{P_{\text {Sample }}}
$$

Where $P_{\text {sample }}$ is the absolute pressure of the sample and $P_{\text {Dryer }}$ is the vapor pressure of the sample after passing through the chiller. $\mathrm{P}_{\text {Dryer }}$ was found using Equation 11 where $\mathrm{T}$ is the temperature measured at the sample dryer. Emissions constituents that were measured wet (THC, NO, NOX) were corrected to a dry measurement by Equation 23.

Equation 23: Wet-to-Dry Correction for Emissions Constituents Measured Wet Derived from Equation 1065.659-1 from CFR Title 40 Part 1065 Subpart G [44]

$$
[\text { Emission }]_{\text {Dry }}=\frac{[\text { Emission }]_{\text {Meas }}}{1-\left[\mathrm{H}_{2} \mathrm{O}\right]}
$$

Where [Emission $]_{\text {meas }}$ is a wet emission measurement and $\left[\mathrm{H}_{2} \mathrm{O}\right]$ is the molar concentration of water in the exhaust. Equation 24 is a correction for the paramagnetic oxygen measurement based on NO interference which was required prior to using it for air-to-fuel ratio calculations [45].

Equation 24: Paramagnetic Oxygen Analyzer Measurement Correction for NO interference [45]

$$
\left[\mathrm{O}_{2}\right]_{\text {Corr }}=\left[\mathrm{O}_{2}\right]_{\text {Meas }}-.442 *[\mathrm{NO}]_{\text {Meas }}
$$

Equation 25 details a method to determine equivalence ratio based on a wet sample measurement of THC, $\mathrm{NO}$ and $\mathrm{NO}_{2}$ and dry sample measurement of remaining emissions constituents. The air-to-fuel ratio was then calculated by dividing the stoichiometric air-to-fuel ratio (Equation 18) by $\Phi$. 


\section{Equation 25: Chemical Balance to Determine Equivalence Ratio [11]}

$$
\phi=\frac{2 * n_{o_{2}}}{n_{P} *\left(\left[\mathrm{H}_{2} \mathrm{O}\right]+[\mathrm{NO}]+2 *\left[\mathrm{NO}_{2}\right]\right)+n_{P} *\left(1-\left[\mathrm{H}_{2} \mathrm{O}\right]\right)\left([\mathrm{CO}]+2 *\left[\mathrm{CO}_{2}\right]+2 *\left[\mathrm{O}_{2}\right]\right)}
$$

Where $\mathrm{n}_{\mathrm{O} 2}$ is the number of oxygen molecules necessary for complete combustion computed by Equation 26 and $n_{p}$ is the total number of moles of exhaust products computed by Equation 27.

Equation 26: Number of Oxygen Molecules Necessary for Complete Combustion [11]

$$
n_{O_{2}}=n_{C, \text { Fuel Mix }}+\frac{n_{H, \text { Fuel Mix }}}{4}
$$

Equation 27: Number of Moles of the Exhaust Products [11]

$$
n_{P}=\frac{n_{C, \text { Fuel Mix }}}{[T H C]+\left(1-\left[\mathrm{H}_{2} \mathrm{O}\right]\right)\left([\mathrm{CO}]+\left[\mathrm{CO}_{2}\right]\right)}
$$

The mole fraction of $\mathrm{H}_{2} \mathrm{O}$ in the exhaust was determined using Equation 28.

Equation 28: Mole Fraction of $\mathrm{H}_{2} \mathrm{O}$ in the Exhaust

$$
\left[\mathrm{H}_{2} \mathrm{O}\right]=\left(\frac{n_{H, \text { Fuel Mix }}}{2 * n_{C, \text { Fuel Mix }}}\right) * \frac{[\mathrm{CO}]+\left[\mathrm{CO}_{2}\right]}{\left[1+\frac{[\mathrm{CO}]}{\left[\mathrm{CO}_{2}\right]}+\left(\frac{n_{H, \text { Fuel Mix }}}{2 * n_{C, \text { Fuel Mix }}}\right) *\left([\mathrm{CO}]+\left[\mathrm{CO}_{2}\right]\right)\right]}
$$

\subsubsection{Exhaust Emissions Calculations}

The mass rate of exhaust emissions were calculated using their individual molecular masses, corrected concentrations and the sum of the intake and fuel flowrates to determine an exhaust flow, which was ultimately converted to a molar flow rate. The molecular masses for each exhaust emission constituent were obtained from CFR Title 40 Part 1065 Subpart K [44], with the exception of THC, which used the molecular mass of $\mathrm{CH}_{4}$ since the exhaust THC emissions were presumed to be predominantly $\mathrm{CH}_{4}$. Additionally, the THC analyzer was spanned using a $\mathrm{CH}_{4}$ and "zero" air bottle. Emissions measurements made after a sample dryer $\left(\mathrm{CO}, \mathrm{CO}_{2}\right.$, and $\left.\mathrm{O}_{2}\right)$ were corrected to a wet measurement for reporting by Equation 29.

Equation 29: Dry Emissions Measurement to Wet Correction from Equation 1065.659-1 from CFR Title 40 Part 1065 Subpart G [44]

$$
[\text { Emission }]_{\text {Wet }}=[\text { Emission }]_{\text {Meas }} *\left(\frac{1-\left[\mathrm{H}_{2} \mathrm{O}\right]}{1-\left[\mathrm{H}_{2} \mathrm{O}\right]_{[\text {Emission }]}}\right)
$$

$\mathrm{NO}$ and $\mathrm{NO}_{\mathrm{x}}$ emissions were corrected based on the mole fraction of water in the engines intake air stream as displayed by Equation 30.

Equation 30: NOx Correction Factor from Equation 1065.670-1 from CFR Title 40 Part 1065 Subpart G [44]

$$
\left[\mathrm{NO} \text { or } \mathrm{NO}_{X}\right]_{\text {Corr }}=\left[\mathrm{NO} \text { or } \mathrm{NO}_{X}\right]_{\text {Meas }} *\left(9.953 *\left[\mathrm{H}_{2} \mathrm{O}\right]_{\text {Intake }}+0.832\right)
$$

The amount of $\mathrm{N}_{2}$ and hydrogen $\left(\mathrm{H}_{2}\right)$ in the exhaust were calculated to determine the molecular mass and subsequently the molecular flowrate of the exhaust. Equation 31 details the calculation of the 
molar concentration of hydrogen in the exhaust based on equilibrium. The molar concentration of $\mathrm{N}_{2}$ was assumed to be the remainder after subtracting the sum of all of the measured and derived exhaust products from one.

\section{Equation 31: Molar Concentration of Hydrogen in the Exhaust [11]}

$$
\left[\mathrm{H}_{2}\right]=\frac{\left[\mathrm{H}_{2} \mathrm{O}\right] *[\mathrm{CO}]_{\text {Dry }}}{\mathrm{K} *\left[\mathrm{CO}_{2}\right]_{\text {Dry }}}
$$

The molecular mass of the exhaust was determined by summing up the molar concentration of each exhaust emissions constituent multiplied by its molecular mass. The molar flow rate of the exhaust was then determined by dividing the mass flow rate of the exhaust by the molecular mass of the exhaust. The mass rate of each emission constituent was then calculated by Equation 32.

\section{Equation 32: Mass Rate of Exhaust Emission Constituent}

$$
\dot{m}_{[\text {Emission }]}=M_{[\text {Emission }]} *[\text { Emission }] * \dot{n}_{\text {Exhaust }}
$$

Where $\mathrm{M}_{\text {Emission }}$ is the molecular mass of the exhaust emission constituent and $\dot{\mathrm{n}}_{\text {Exhaust }}$ is the molar flow rate of the exhaust. Brake specific emissions were found by integrating this value over the duration of the test segment and dividing it by the measured work output by the engine.

\subsubsection{Combustion Efficiency and Brake Thermal Efficiency}

Combustion efficiency was calculated using the mass fractions of $\mathrm{CO}, \mathrm{THC}$ and $\mathrm{H}_{2}$ in the exhaust and the fuel and intake flow detailed by Equation 33. Soot emissions may also be included in Equation 33, but their magnitude in comparison to $\mathrm{THC}, \mathrm{CO}$ and $\mathrm{H}_{2}$ is small, especially for $\mathrm{RCCl}$ combustion, that the effect on combustion efficiency is assumed to be negligible.

\section{Equation 33: Combustion Efficiency [11]}

$$
\text { Combustion Efficiency }=1-\frac{X_{C O} * Q_{H V_{C O}}+X_{T H C} * Q_{H V_{T H C}}+X_{H_{2}} * Q_{H V_{H 2}}}{\left[\dot{m}_{\text {Fuel }} /\left(\dot{m}_{\text {Fuel }}+\dot{m}_{\text {Intake }}\right)\right] * Q_{H V_{\text {Fuel Mix }}}}
$$

Where $X_{\text {[Emission] }}$ is the mass fraction and $Q_{H v}$ is the lower heating value. The equivalent lower heating value for the fuel is determined by Equation 34.

\section{Equation 34: Lower Heating Value of the Fuel Mixture}

$$
Q_{H V_{\text {Fuel Mix }}}=\frac{Q_{H V_{C N G}} * \dot{m}_{C N G}+Q_{H V_{\text {Diesel }}} * \dot{m}_{\text {Diesel }}}{\dot{m}_{C N G}+\dot{m}_{\text {Diesel }}}
$$

The BTE of the engine can be calculated using Equation 35.

\section{Equation 35: Brake Thermal Efficiency [11]}

$$
B T E=\frac{W_{B}}{\text { Combustion Efficiency } * \dot{m}_{\text {Fuel Mix }} * Q_{H V_{\text {Fuel Mix }}}}
$$

Where $W_{B}$ is the brake work produced by the engine. The brake FCE is the product of combustion efficiency and BTE. 
Equation 36: Fuel Conversion Efficiency [11]

$$
\mathrm{FCE}=\text { Combustion Efficiency } * \text { BTE }=\frac{W_{B}}{\dot{m}_{\text {Fuel Mix }} * Q_{H V_{\text {Fuel Mix }}}}
$$




\section{Experimental Approach}

Experiments to quantify high reactivity fuel property effects on $\mathrm{RCCl}$ combustion were conducted at one engine speed and two different engine loads. The selected engine speed was 2100 RPM for all experiments presented herein based on previous LTC research performed by the author with this engine and the FACE diesel fuels. This engine speed was also near the intermediate speed of 2000 RPM. Low load experiments in this effort were performed targeting $40 \mathrm{ft}-\mathrm{lb}$ of torque, or 3.6 bar BMEP. High load experiments were performed at a target of $67 \mathrm{ft}-\mathrm{lb}$, or approximately 6 bar BMEP. A higher load was sought for the high load experiments, but it was ultimately found that loads greater than 6 bar BMEP at this engine speed approached, and in certain instances exceeded in-cylinder PRRs of $10 \mathrm{bar} / \mathrm{CA}^{\circ}$ for some of the high reactivity fuels evaluated, which was deemed potentially detrimental to the engine. Curran, et al. also observed approximately 6 bar BMEP to be the maximum achievable load based on PRR at a similar engine speed (2000 RPM) when operating the same model engine in RCCI mode [8]. The engine speed and load points selected for RCCI research are shown in Figure 12 on a map of the maximum torque versus speed during CDC operation.

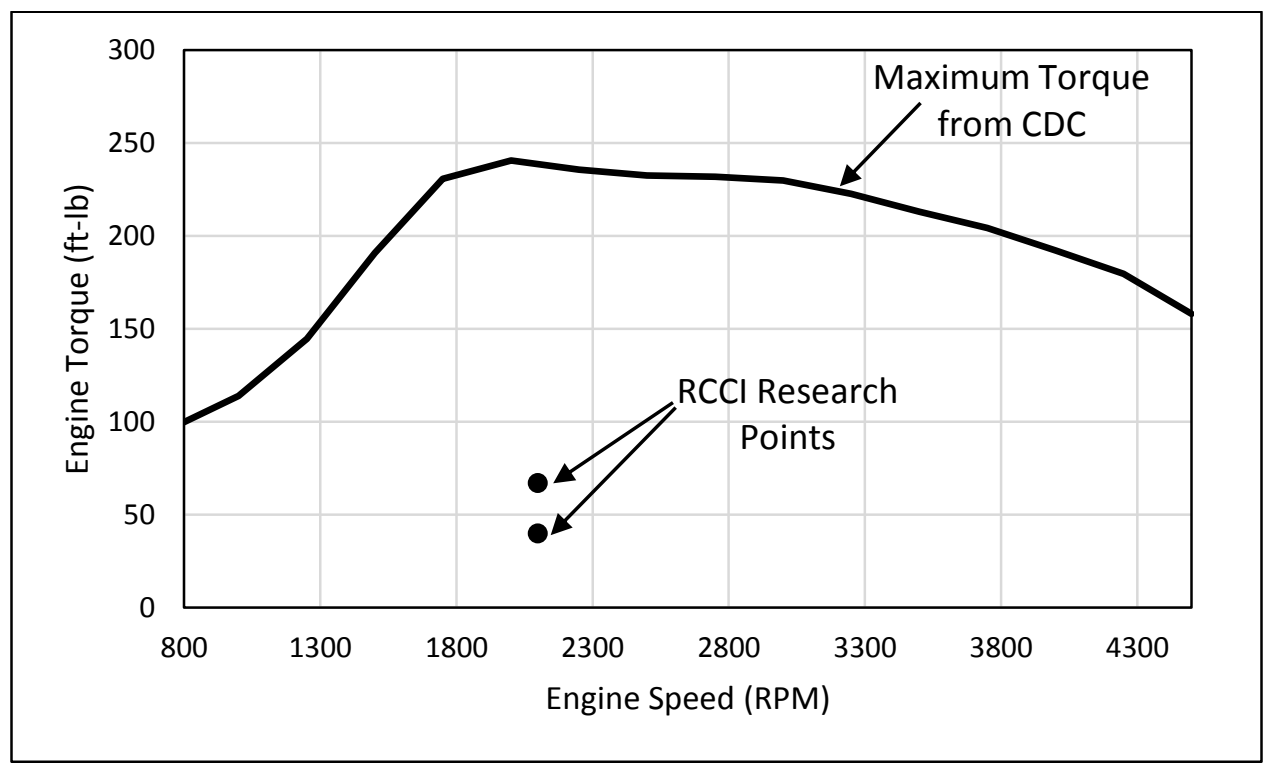

Figure 12: $\mathrm{RCCl}$ Research Points in Relation to Maximum Torque versus Speed from CDC Operation

The test engine and Drivven engine controller offered a high level of flexibility and control. In addition to this level of control, the technology of this engine provided an array of control variables that affect its performance and emissions characteristics. The interactions of the control variables were further compounded by their interactions with the fuel properties. This presents a situation where the engine may operate as desired for one fuel, but not as desired for another fuel with differing properties even though the set points of these control variables remain the same. Thus, it was important to explore and substantiate the effects of these control variables to determine which variables had the most profound effect on the performance and emissions characteristics of the test engine. With that knowledge, tests matrices were formed that covered the important control parameters in an effort to exploit the properties of a given fuel. 
Initial parameters of interest for this study were:

- PFI Duration*

- Start of injection timing of direct injected fuel or high reactivity fuel (DI SOI)

- Number of direct injections per cycle

- Common rail fuel pressure

- EGR rate

- Intake manifold air temperature (IMAT)

- IMAP

- Swirl valve positions

* Note that PFI duration was replaced with Percent of natural gas comprising total fuel injected on an energy basis (percentage CNG) after the low load parametric study

The shape or rate profile of the injection would also have been a parameter of interest; however, the Drivven controller did not offer this capability. The amount of natural gas that was injected was controlled by setting a fixed injection duration for the PFI. The diesel-fueling rate into the cylinder was then controlled to meet the desired engine load. Percentage CNG was defined as the percent of energy injected as natural gas (CNG) divided by the total fuel energy (diesel and CNG) injected into the engine. The start of the diesel injection timing, common rail fuel pressure, and swirl valve position were set to fixed values (versus a value from the look up tables as found in a production engine) in the Drivven engine controller software. EGR rate was controlled by varying the position of the EGR valve based on feedback from zirconium oxygen sensors in the intake manifold and exhaust pipe. Note, however, EGR was ultimately deemed unnecessary to reduce in-cylinder PRR and $\mathrm{NO}_{\mathrm{x}}$ emissions for the low load operating condition. At the high load operating condition, EGR significantly increased the intake manifold temperature, which advanced combustion phasing and was detrimental to RCCl operation. Subsequently, no EGR was used at either load point. IMAT was controlled by adjusting the cooling water flow through an air-to-water heat exchanger positioned between the turbocharger compressor outlet and intake manifold inlet. IMAP was controlled by a PID controller within the Drivven software that altered the position of the VGT rack.

\subsection{Scoping and Cylinder-to-Cylinder Balancing}

A review of literature and publications from organizations also using the GM Z19DTH engine platform for $\mathrm{RCCl}$ research provided a starting point for the values of the control parameters. Through experimentation, relatively low $\mathrm{NO}_{x}$ and soot emissions, and thermal efficiency comparable to diesel only operation was achieved. Initial values for the parameters of interest and the operating conditions are presented in Table 6.

Table 6: Initial Parameter Settings for RCCI Combustion

\begin{tabular}{|c|c|c|c|c|c|c|c|c|}
\hline $\begin{array}{c}\text { Desired } \\
\text { Engine } \\
\text { Speed } \\
\text { (RPM) }\end{array}$ & $\begin{array}{c}\text { Desired } \\
\text { Engine } \\
\text { Torque } \\
\text { (ft-lb) }\end{array}$ & $\begin{array}{c}\text { Brake } \\
\text { Mean } \\
\text { Effective } \\
\text { Pressure } \\
\text { (bar) }\end{array}$ & $\begin{array}{c}\text { Diesel } \\
\text { Main } \\
\text { SOI } \\
\left({ }^{\circ} \text { BTDC) }\right.\end{array}$ & $\begin{array}{c}\text { Rail } \\
\text { Pressure } \\
\text { (bar) }\end{array}$ & $\begin{array}{c}\text { PFI } \\
\text { Duration } \\
\left(\text { CA }{ }^{\circ}\right)\end{array}$ & $\begin{array}{c}\text { Swirl } \\
\text { Valve } \\
\text { Position }\end{array}$ & $\begin{array}{c}\text { EGR } \\
\text { Rate (\% } \\
\text { Volume) }\end{array}$ & $\begin{array}{c}\text { VGT } \\
\text { Rack } \\
\text { Position }\end{array}$ \\
\hline 2100 & 40 & 3.6 & 50 & 500 & 12 & $\begin{array}{c}\text { Fully } \\
\text { Open }\end{array}$ & 0 & $\begin{array}{c}\text { Fully } \\
\text { open }\end{array}$ \\
\hline
\end{tabular}


Initial emissions and performance results at these operating were obtained using chemically pure $\mathrm{CH}_{4}$ in compressed gas cylinders. The data from these results were recorded while laboratory equipment was still being debugged and calibrated, thus the emissions and performance data of these initial tests are not presented. Regardless of the data quality, it was evident that a high level of variation in the engine performance and emissions existed. This was presumed to be a result of cylinder-to-cylinder imbalances, which have been documented by Curran, et al. [18] and Yun, et al. [46] while operating four cylinder engines in $\mathrm{RCCl}$ and $\mathrm{HCCl}$ modes.

A baseline measurement of the in-cylinder pressure during $\mathrm{RCCl}$ combustion was obtained for each cylinder while operating the engine at the initial parameter settings listed in Table 6. Due to the limitation of only one in-cylinder pressure transducer, these measurements were taken consecutively rather than simultaneously. The solid lines and "Pre" designation in Figure 13 represent these initial incylinder pressure measurements with no balancing adjustments. The data plotted in the following figures were the average of 300 consecutive cycles. The average maximum pressure measured among the cylinders varied considerably, noting that the maximum pressure displayed for cylinder $3(6,776 \mathrm{kPa})$ was nearly half of that for cylinder $1(12,145 \mathrm{kPa})$.

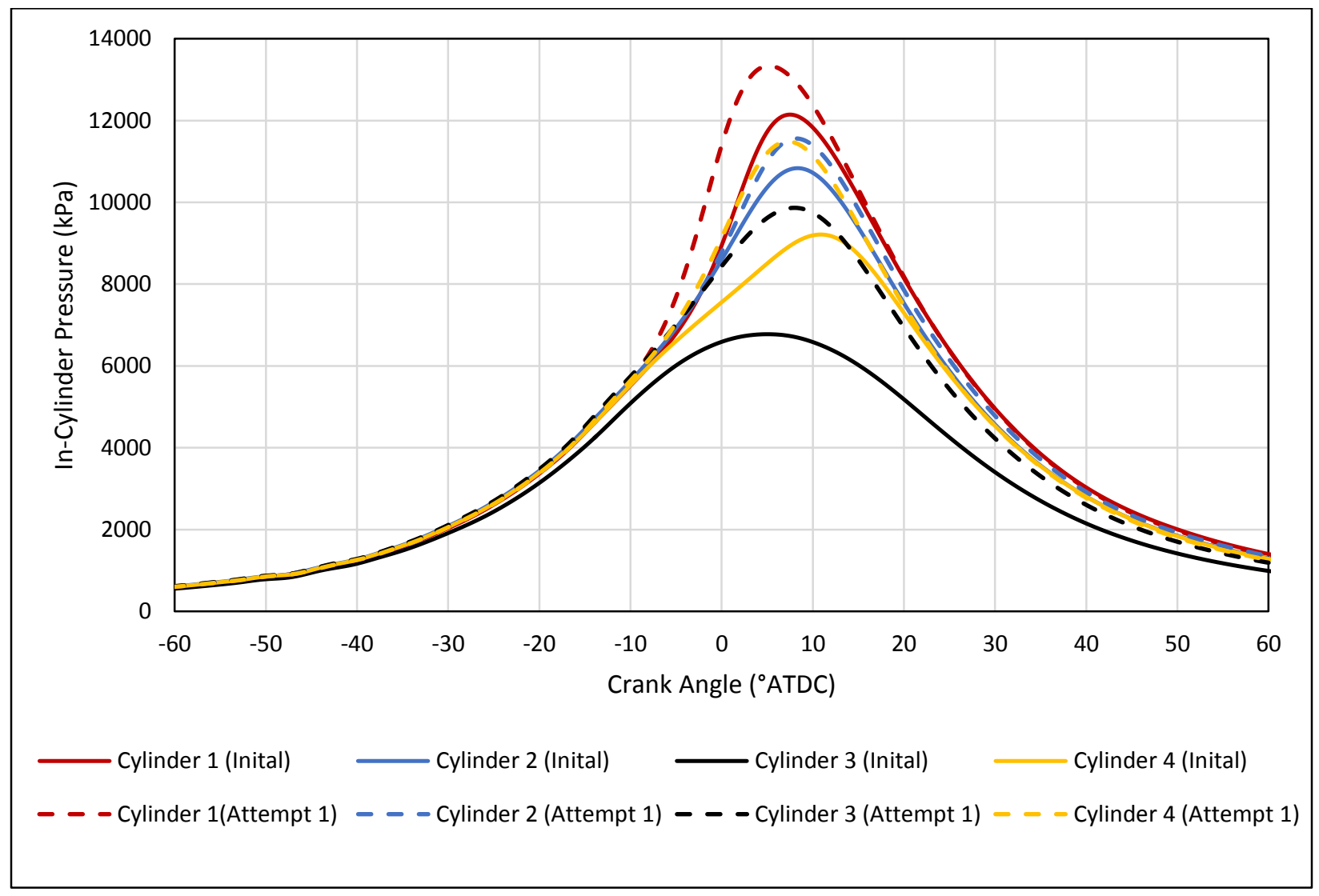

Figure 13: In-Cylinder Pressure Measurements for Cylinder Balancing Efforts with Chemically Pure $\mathbf{C H}_{4}$

The first effort to balance the in-cylinder pressure among the cylinders involved multiplying the duration of the PFI for cylinders 3 and 4 by a coefficient. The coefficients used were 0.8333 and 0.9167 for cylinders 3 and 4, respectively. The results after applying these coefficients are represented by the dashed lines and "Attempt 1" labels in Figure 13. The PFI duration for cylinders 1 and 2 remained 
unchanged. The result was an increase in maximum pressure not just for cylinders 3 and 4 but also for cylinders 1 and 2. The increased pressure in cylinders 1 and 2 was a result of increased diesel fueling for all cylinders in order to achieve the engine load set point as the $\mathrm{CH}_{4}$ fueling was reduced in cylinders 3 and 4.

To continue testing without using costly chemically pure $\mathrm{CH}_{4}$ cylinders, a refillable $\mathrm{CNG}$ tank was installed. The tank was filled with CNG from a local refueling station. The parameter settings for RCCI remained the same as those in Table 6 with the exception of the PFI duration, which was reduced to 10 $\mathrm{CA}^{\circ}$. To reduce the maximum pressure in cylinder 1 , a new approach was taken to alter the DI duration (diesel). For "Attempt 2," coefficients of 0.8200 and 0.9167 were multiplied by the PFI duration of cylinders 3 and 4, respectively, and a coefficient of 0.9000 was multiplied by the DI duration of cylinder 1. Displayed by the solid lines in Figure 14, this configuration reduced the maximum pressure of cylinder 1 to be on the order of cylinder 2 and 4 , but cylinder 3 still exhibited a maximum pressure that was nearly $2,000 \mathrm{kPa}$ lower than the other cylinders. The final attempt at cylinder balancing used the previous settings with the exception of a coefficient of 1.1000 multiplied by the DI duration of cylinder

3. The final coefficients used for this attempt and throughout the rest of this experiment are displayed in Table 7. These coefficients resulted in all the cylinders having an average maximum in-cylinder pressure within $415 \mathrm{kPa}$ of each other. Cylinder 3 now exhibited the highest maximum pressure at $11,675 \mathrm{kPa}$, while the cylinder 4 exhibited the lowest at 11,260 kPa.

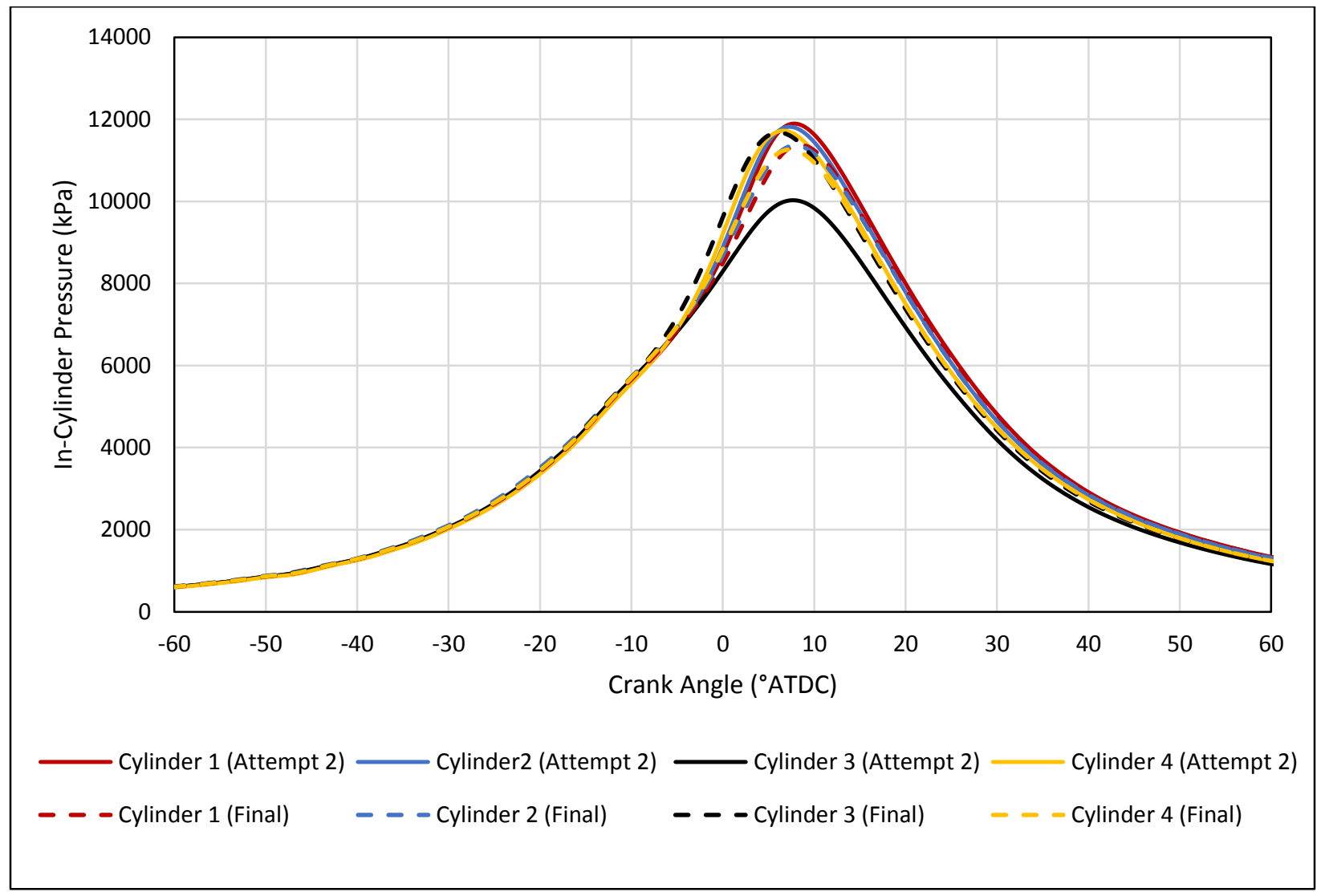

Figure 14: In-Cylinder Pressure Measurements for Cylinder Balancing Efforts with CNG 
Table 7: Final Injection Duration Coefficients for Cylinder Balancing

\begin{tabular}{|c|c|c|}
\hline Cylinder & $\begin{array}{c}\text { DI Duration } \\
\text { Coefficient }\end{array}$ & $\begin{array}{c}\text { PFI Duration } \\
\text { Coefficient }\end{array}$ \\
\hline 1 & 0.9000 & 1.0000 \\
\hline 2 & 1.0000 & 1.0000 \\
\hline 3 & 1.1000 & 0.8200 \\
\hline 4 & 1.0000 & 0.9167 \\
\hline
\end{tabular}

To give some measure of confidence that these balancing efforts could be applied to different conditions a set of tests were performed at a PFI duration of $9.25 \mathrm{CA}^{\circ}$. The in-cylinder pressure traces from these tests are shown in Figure 15. The result was all the cylinders producing an average maximum in-cylinder pressure within $474 \mathrm{kPa}$ of each other. Cylinder 2 exhibited the highest maximum pressure at $12,593 \mathrm{kPa}$, while cylinder 4 exhibited the lowest at $12,119 \mathrm{kPa}$.

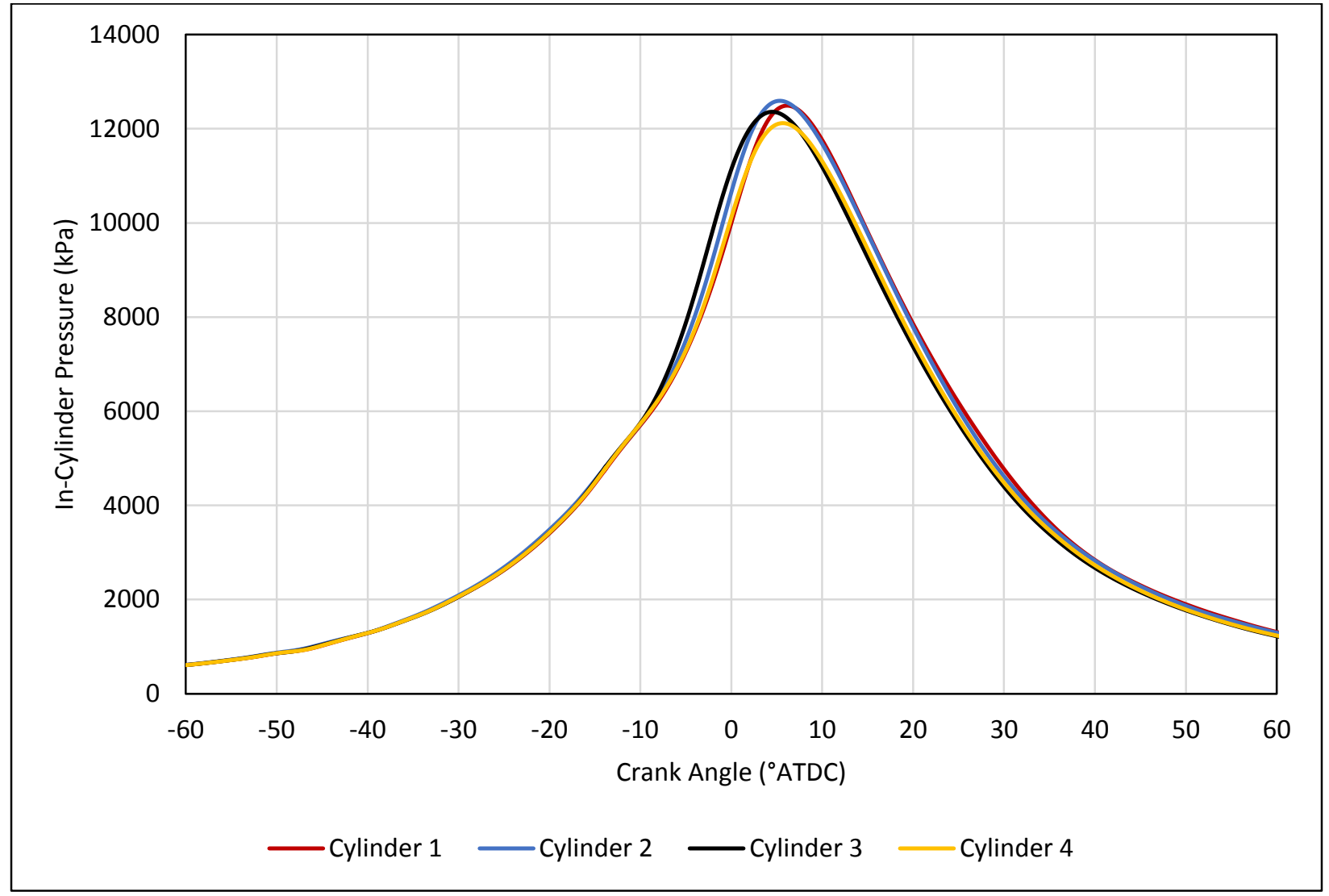

Figure 15: In-Cylinder Pressure Measurements for $9.25^{\circ}$ PFI Duration with CNG

Based on the limited high reactivity fuel available for testing and the availability of only one in-cylinder pressure transducer the cylinder balancing coefficients presented in Table 7 were used for all of the low and high load experiments. Combustion data presented for the remaining experiments was obtained from cylinder 3 since it had the highest DI coefficient and lowest PFI coefficient. 


\subsection{Statistical Analysis}

To address the hypotheses presented in Chapter 3 it was necessary to make determinations on which high reactivity fuel properties affect $\mathrm{RCCl}$ combustion, exhaust emissions, and fuel consumption. In order to make these determinations, data was gathered and analyzed. Test matrices were developed to gather this data. To develop these test matrices it was necessary to make determinations on which engine control parameters, such as those presented at the beginning of Chapter 5, had the largest effect on $\mathrm{RCCl}$ combustion, exhaust emissions, and fuel consumption. JMP is a statistical software that was used to aid in making determinations on engine control parameter effects and high reactivity fuel property effects on RCCI [47].

Making a determination on whether a property or parameter is truly influential on a process ( $\mathrm{RCCl}$ in the case of this research) can be discussed in terms of two hypotheses. One hypothesis, often referred to as the null hypothesis, would represent the notion that a parameter has no effect on the process being scrutinized. Freund, et al. states, "The null hypothesis is a statement about the values of one or more parameters. This hypothesis represents the status quo and is usually not rejected unless the sample results strongly imply that is false" [48]. For example, a null hypothesis for this research could be that $\mathrm{CN}$ has no effect on $\mathrm{RCCl}$ combustion efficiency. To reject such a hypothesis would be to state that $\mathrm{CN}$ does have an effect on $\mathrm{RCCl}$ combustion efficiency, which contradicts the null hypothesis. As stated by Freund, et al. "the alternative hypothesis is a statement that contradicts the null hypothesis. This hypothesis is accepted if the null hypothesis is rejected" [48]. Thus, if it was proven that CN does have an effect on $\mathrm{RCCl}$ combustion efficiency, the alternative hypothesis was accepted. Of course, in order to accept or reject a hypothesis, results or data must exist that contradict the hypothesis. These results may only be a sample of all possible combinations or configurations and are often referred to as sample statistics. This leads to the rejection region, which is "the range of values of a sample statistic that will lead to rejection of the null hypothesis" [48]. To define this rejection region two types of errors must be recognized. A type I error is defined by Freund, et al. as incorrectly rejecting the null hypothesis or when the null hypothesis "is actually true and our sample based inference procedures reject it" [48]. A type II error occurs when the null hypothesis is in fact not true, but "our inference procedure fails to detect this fact" [48]. The probability of making one of these errors can then be used as the basis to form the rejection region.

In practice, the probability of making a type I error is used to define the rejection region over the probability of making a type II error. This is related to the selection of the null hypothesis and it being the status quo. There is less risk in accepting the status quo rather than rejecting it. For example, stating that $\mathrm{CN}$ influences combustion efficiency when it really does not (type I error) is more detrimental than stating that it does not (type II error) when it actually does. In order to accept or reject the null hypothesis a maximum value for the probability of making a type I error must be established. This maximum value is referred to as the significance level by Freund, et al [48]. These values are often chosen to be $0.10,0.05$, or 0.01 . However, it can be difficult to know what significance level should be chosen in order to reject the null hypothesis. An alternative approach is to use a p-value, which allows for the results of a significance test to be reported without defining a level of significance. "The $p$-value is the probability of committing a type I error if the actual sample value of the statistic is used as the boundary of the rejection region" [48]. Additionally, "a small p-value is an indication that the null hypothesis is false" [49]. As related to the example provided previously, a small p-value obtained from a 
significance test of $\mathrm{CN}$ on combustion efficiency would provide confidence that $\mathrm{CN}$ is in fact influential on combustion efficiency.

\subsection{Parametric Study}

A series of preliminary experiments were performed using locally sourced "pump" diesel and CNG fuels to determine which engine control parameters had the greatest influence on $\mathrm{RCCl}$ exhaust emissions and performance results. This was necessary to reduce the number of parameters that would be varied for the final high reactivity fuels comparison testing as well as preserve the limited quantities of the FACE diesel fuels for the most important experiments. It is noted that limited quantities of the FACE fuels were available for this effort. To aid in this process, the JMP statistical software was used [47]. The parameters of interest presented at the beginning of Chapter 5, with the exception of EGR rate, were used as the input variables for design of experiments (DOE) screening matrices in JMP.

\subsubsection{Low Load Operating Condition Parametric Study}

Preliminary activities at the low load operating condition, as well as information from the literature review, helped determine the values and number of levels used for each input variable displayed in Table 8. Additionally, since RCCl was achievable at this load with a single DI event, multiple injections were not investigated in order to reduce the complexity of the DOE matrix.

Table 8: Input Variable Levels and Values for the Low Load Parametric Study

\begin{tabular}{|c|c|c|}
\hline Input Variable & Levels & Values \\
\hline IMAT $^{\circ} \mathrm{C}$ ) & 3 & $37,39,41$ \\
\hline Number of Injections $^{\circ}$ & 1 & 1 \\
\hline DI SOI (CA $\left.^{\circ} \mathrm{BTDC}\right)$ & 3 & $45,50,55$ \\
\hline PFI Duration (CA ${ }^{\circ}$ ) & 3 & $9,10,11$ \\
\hline Rail Pressure (bar) & 2 & 400,600 \\
\hline Swirl Valve Position (\% duty cycle) & 2 & 55,93 \\
\hline IMAP (bar) & 2 & $1.4,1.5$ \\
\hline
\end{tabular}

A full factorial matrix to analyze all levels of each variable would result in 216 individual tests. Due to limitations of time, fuel, and other laboratory consumables a decision was made to implement a fractional factorial matrix. Two options were provided by JMP, which were a main effects screen design with a minimum of 12 runs or a mixed level specialized orthogonal-array design titled L18 Chakravarty. The L18 Chakravarty design can have up to three, two-level factors, and six, three-level factors and was the approach taken based on retaining orthogonality of the test matrix with multi-level factors. The DOE matrix developed by JMP and used for the low load operating condition parametric study is shown in Table 9. 
Table 9: L18 Chakravarty DOE Test Matrix for the Low Load Parametric Study

\begin{tabular}{|c|c|c|c|c|c|c|}
\hline Test \# & IMAT ( $\left.^{\circ} \mathbf{C}\right)$ & $\begin{array}{c}\text { DI SOI (CA } \\
\text { BTDC) }\end{array}$ & $\begin{array}{c}\text { PFI } \\
\text { Duration } \\
\left(\text { CA }^{\circ}\right)\end{array}$ & $\begin{array}{c}\text { Rail } \\
\text { Pressure } \\
\text { (bar) }\end{array}$ & Swirl (\%) & IMAP (bar) \\
\hline 1 & 37 & 50 & 10 & 400 & 93 & 1.5 \\
\hline 2 & 37 & 45 & 11 & 600 & 93 & 1.4 \\
\hline 3 & 37 & 55 & 10 & 600 & 55 & 1.5 \\
\hline 4 & 41 & 45 & 10 & 600 & 93 & 1.4 \\
\hline 5 & 37 & 50 & 9 & 600 & 93 & 1.4 \\
\hline 6 & 41 & 55 & 9 & 600 & 93 & 1.4 \\
\hline 7 & 39 & 55 & 11 & 600 & 93 & 1.4 \\
\hline 8 & 41 & 45 & 11 & 400 & 93 & 1.5 \\
\hline 9 & 41 & 50 & 9 & 400 & 93 & 1.5 \\
\hline 10 & 41 & 55 & 10 & 400 & 55 & 1.4 \\
\hline $\mathbf{1 1}$ & 39 & $\mathbf{4 5}$ & 9 & $\mathbf{6 0 0}$ & $\mathbf{5 5}$ & $\mathbf{1 . 5}$ \\
\hline 12 & 37 & 55 & 11 & 400 & 93 & 1.5 \\
\hline 13 & 39 & 50 & 10 & 600 & 93 & 1.4 \\
\hline 14 & 39 & 55 & 9 & 400 & 93 & 1.5 \\
\hline 15 & 41 & 50 & 11 & 600 & 55 & 1.5 \\
\hline $\mathbf{1 6}$ & $\mathbf{3 7}$ & $\mathbf{4 5}$ & $\mathbf{9}$ & $\mathbf{4 0 0}$ & $\mathbf{5 5}$ & $\mathbf{1 . 4}$ \\
\hline 17 & 39 & 50 & 11 & 400 & 55 & 1.4 \\
\hline 18 & 39 & 45 & 10 & 400 & 93 & 1.5 \\
\hline
\end{tabular}

Unfortunately, not all of the tests in the matrix could be completed due to maximum PRRs exceeding 10 bar/CA ${ }^{\circ}$. This occurred for two tests, 11 and 16, both of which were at the lowest PFI duration. They are bold and have a strike through them in Table 9. The first step in examining the results from the parametric study was to generate screening reports with JMP. The primary dependent $(Y)$ variables to base the final test matrix from were selected to be brake specific (BS) NOx emissions, BSSoot emissions, and BTE. BSNOx and BSSoot were selected based on the cost, complexity, and volume of exhaust aftertreatment systems that are currently necessary for compression ignition engines as discussed in Chapter 1. BTE was selected based on literature stating that $\mathrm{RCCl}$ is capable of achieving low $\mathrm{NO}_{\mathrm{x}}$ and soot emissions with high thermal efficiencies as presented in Section 2.3.1. However, note that FCE was substituted for BTE during the high load operating condition parametric study and discussion of the results because it was deemed a better metric for $\mathrm{RCCl}$ efficiency, which is detailed in subsequent sections. BSCO, BSTHC, and combustion efficiency were selected to be secondary variables for determining parameters for the final test matrix. Initially, the six parameters of interest were used as the independent $(X)$ variables to generate the screening report. The screening reports provided a contrasts outline, which lists the model effects, a contrast value, Lenth t-ratio, and individual and simultaneous $p$-values. Ideally, an influential effect would be signified by a high absolute value for contrast, a Lenth t-ratio that is significant at the 0.10 level (blue lines) and relatively small individual $p$ values and simultaneous $p$-values. P-values less than 0.05 (red letters) are treated as "fairly small, indicating significance," while $p$-values less than .01 (orange letters) are "small enough to indicate very 
convincing significance" [47]. Two examples of contrasts outlines generated with JMP for BSNO ${ }_{x}$ and BTE are presented in Figure 16 and Figure 17, respectively. The high contrast and Lenth t-Ratio suggest that the rail pressure term in Figure 16 is an influential factor for $\mathrm{BSNO}_{x}$, but the lack of low p-values does not provide confidence in this assumption. Conversely, the values represented in Figure 17 suggest that there was a strong possibility that IMAP and PFI duration influenced BTE.

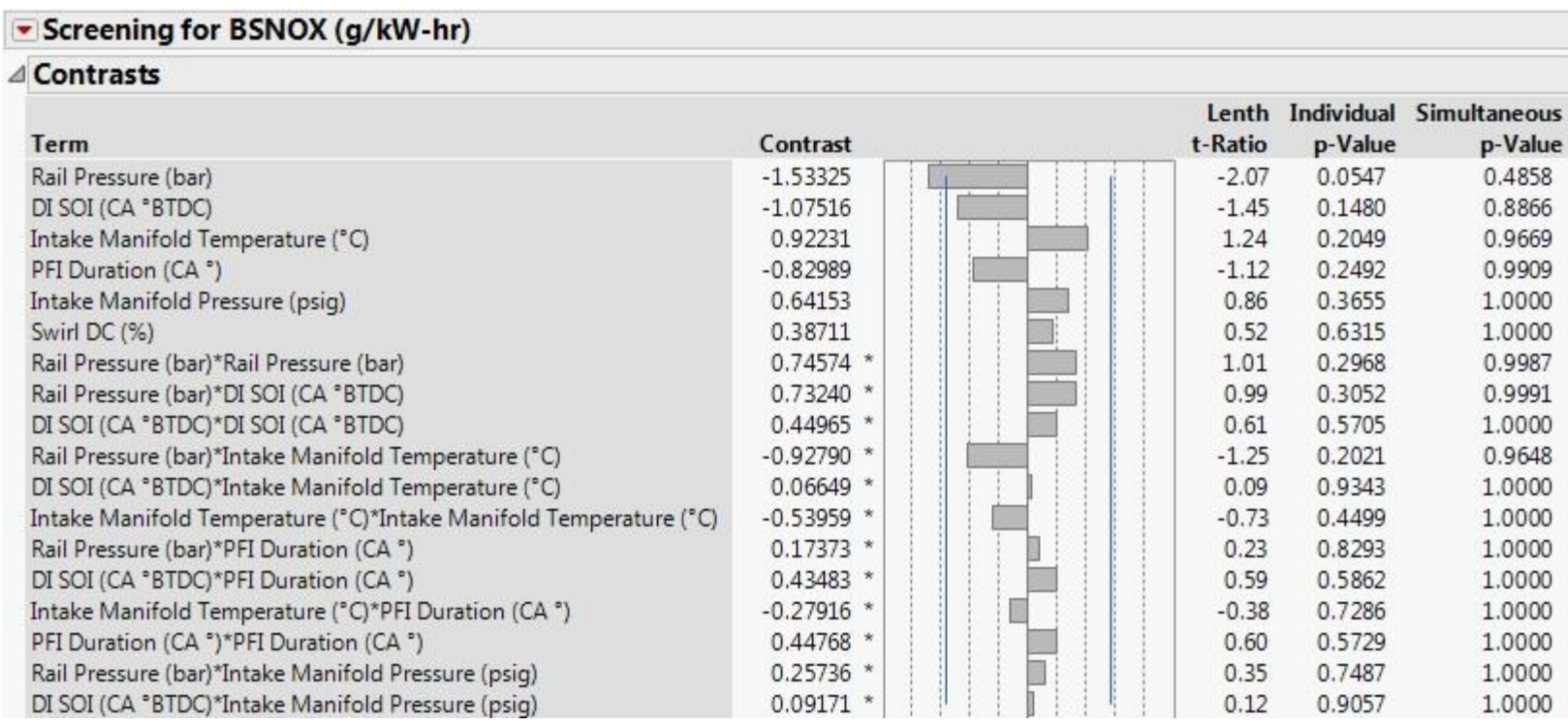

Figure 16: JMP Contrasts Outline for BSNOX Screening Report

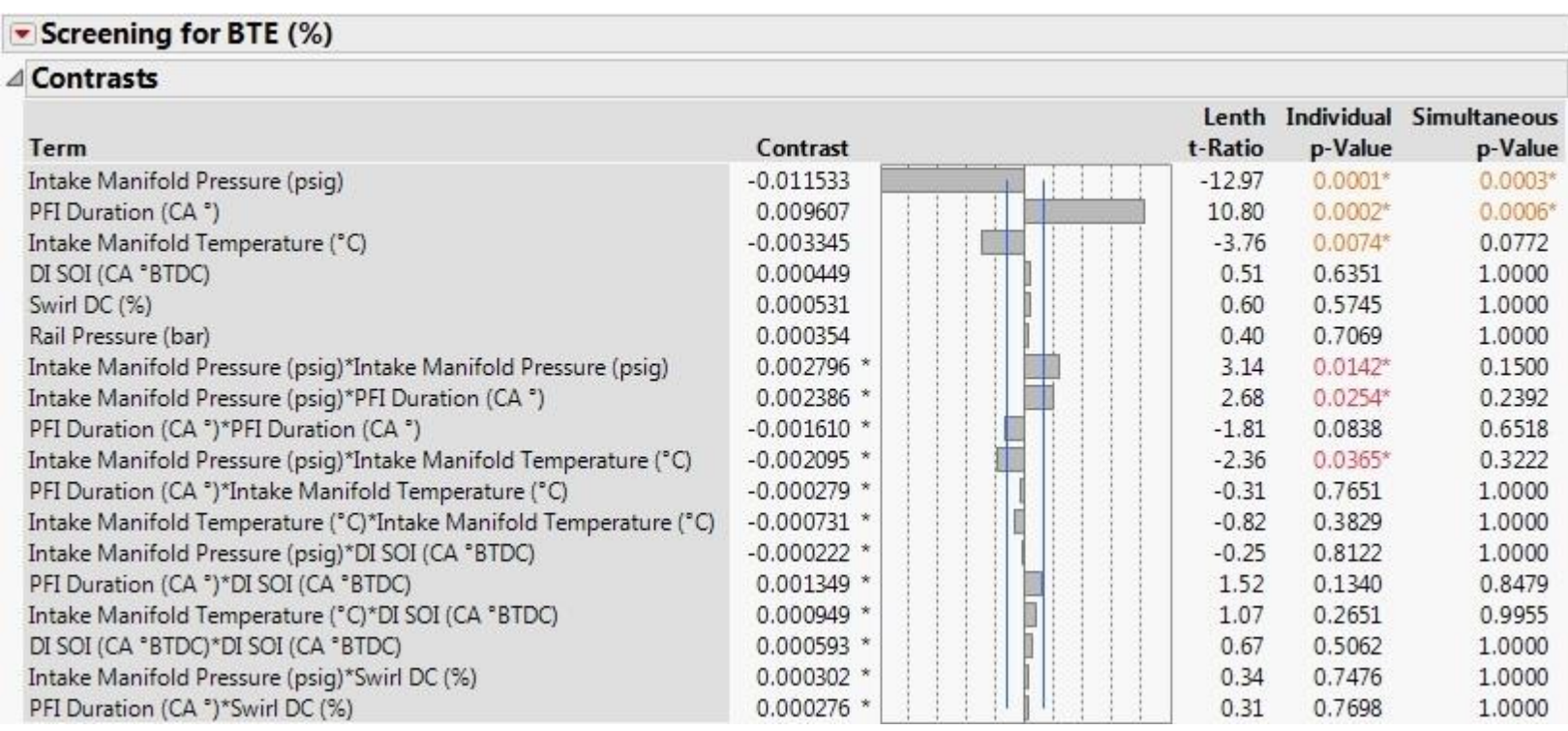

Figure 17: JMP Contrasts Outline for BTE Screening Report

A summary of the other effects that had individual $p$-values less than 0.05 are presented in Table 10 . PFI duration appears to be an influential parameter on BSTHC and combustion efficiency. Note that combustion efficiency is partially a function of THC emissions. From the screening report values presented in Table 10, it was concluded that PFI duration and intake manifold pressure have a strong 
influence on $\mathrm{RCCl}$ at this engine speed and load. P-values less than .05 are bold, while $p$-values less than .01 are bold and underlined.

Table 10: Potentially Influential Parameters from JMP Screening Report Contrasts Outline

\begin{tabular}{|c|c|c|c|c|c|c|}
\hline Effect & Parameter & Contrast & \multicolumn{2}{|c|}{$\begin{array}{c}\text { Lenth } \\
\text { t-Ratio }\end{array}$} & $\begin{array}{c}\text { Individual } \\
\mathbf{p} \text {-value }\end{array}$ & $\begin{array}{c}\text { Simultaneous } \\
\mathbf{p} \text {-value }\end{array}$ \\
\hline BSNOX & \multicolumn{4}{|c|}{ No parameters with p-values less than .05 } \\
\hline BSSoot & IMAP & 2.08 & 2.99 & $\mathbf{0 . 0 2 2 1}$ & 0.176 \\
\hline BTE & IMAP & -0.0115 & -13.0 & $\underline{\mathbf{0 . 0 0 0 1}}$ & $\underline{\mathbf{0 . 0 0 0 3}}$ \\
\hline BTE & PFI Duration & 0.00961 & 10.8 & $\underline{\mathbf{0 . 0 0 0 2}}$ & $\underline{\mathbf{0 . 0 0 0 6}}$ \\
\hline BTE & IMAT & -0.00335 & -3.76 & $\underline{\mathbf{0 . 0 0 7 4}}$ & 0.0772 \\
\hline BTE & IMAP*IMAP & 0.00280 & 3.14 & $\mathbf{0 . 0 1 4 2}$ & 0.150 \\
\hline BTE & $\begin{array}{c}\text { IMAP*PFI } \\
\text { Duration }\end{array}$ & 0.00239 & 2.68 & $\mathbf{0 . 0 2 5 4}$ & 0.239 \\
\hline BTE & IMAP*IMAT & -0.00210 & -2.36 & $\mathbf{0 . 0 3 6 5}$ & 0.322 \\
\hline BSCO & Rail Pressure & 3.39 & 2.35 & $\mathbf{0 . 0 3 6}$ & 0.328 \\
\hline BSTHC & PFI Duration & 7.06 & 6.58 & $\underline{\mathbf{0 . 0 0 0 3}}$ & $\underline{\mathbf{0 . 0 0 6 0}}$ \\
\hline $\begin{array}{c}\text { Combustion } \\
\text { Efficiency }\end{array}$ & PFI Duration & -0.0331 & -7.73 & $\underline{\mathbf{0 . 0 0 0 2}}$ & $\underline{\mathbf{0 . 0 0 2 5}}$ \\
\hline $\begin{array}{c}\text { Combustion } \\
\text { Efficiency }\end{array}$ & IMAP & 0.0159 & 3.71 & $\mathbf{0 . 0 0 6 1}$ & 0.0785 \\
\hline
\end{tabular}

Further analysis of the data revealed that in many cases CA50 occurred before TDC which can have a profound effect on $\mathrm{NO}_{x}$ emissions and BTE. This is evidenced in Figure 18 and Figure 19 where a CA50 occurring before TDC resulted in an exponential increase in $\mathrm{BSNO}_{x}$ emissions while BTE decreased linearly as CA50 occurred before TDC. To further investigate influential parameters and evaluate the perceived influences from Table 10, CA50 was included as an additional parameter and the screening model was re-run. The results, presented in Table 11 agree with results in Figure 18 and Figure 19, and indicate that CA50 was potentially influential on almost all the effects, but also reaffirms that PFI duration and IMAP have an influence on $\mathrm{RCCl}$ at this operating condition. Note that CA50 was measured in $\mathrm{CA}^{\circ}$ after top dead center (ATDC). 


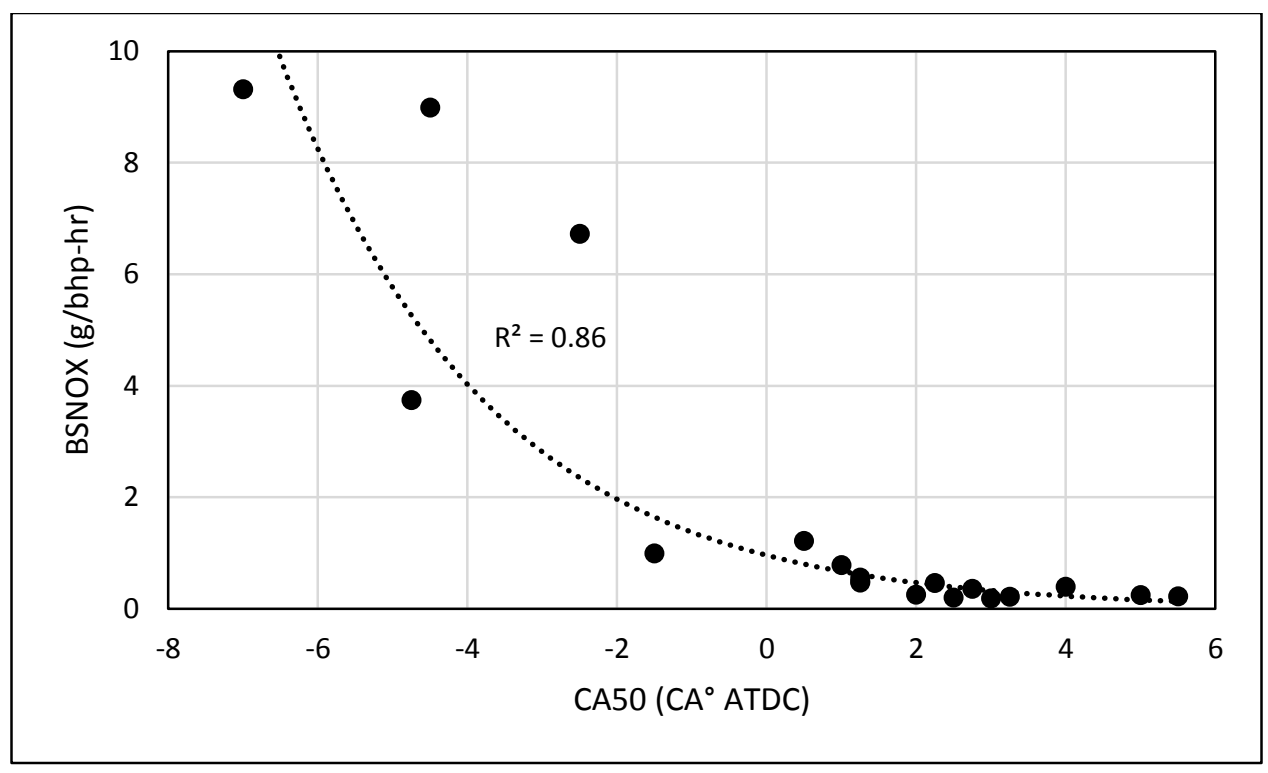

Figure 18: CA50 versus $B N O_{x}$ for Parametric Study

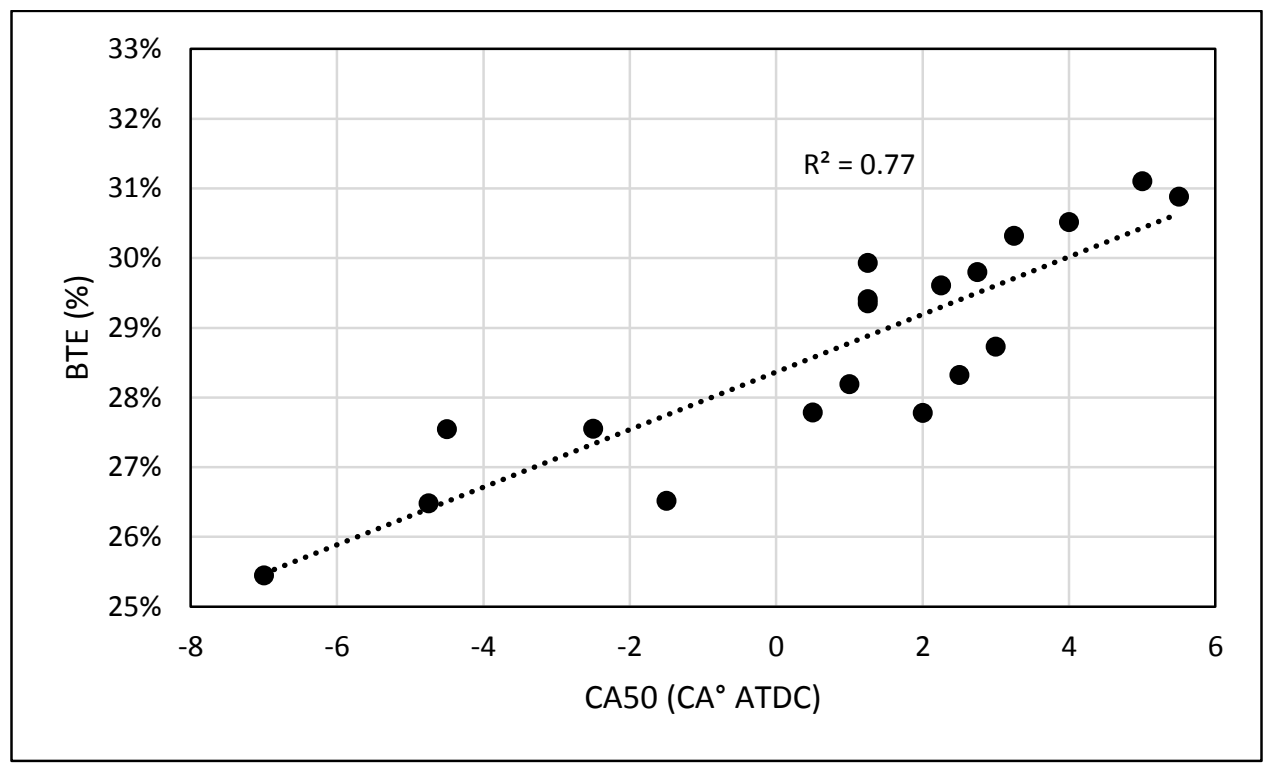

Figure 19: CA50 versus BTE for Parametric Study 
Table 11: Potentially Influential Parameters from JMP Screening Report Contrasts Outline with CA50 Included

\begin{tabular}{|c|c|c|c|c|c|}
\hline Effect & Parameter & Contrast & $\begin{array}{c}\text { Lenth t- } \\
\text { Ratio }\end{array}$ & $\begin{array}{c}\text { Individual } \\
\mathbf{p} \text {-value }\end{array}$ & $\begin{array}{c}\text { Simultaneous } \\
\mathbf{p} \text {-value }\end{array}$ \\
\hline BSNOX & CA50 & -2.53 & -24.3 & $\underline{\mathbf{L . 0 0 0 1}}$ & $\underline{\mathbf{< . 0 0 0 1}}$ \\
\hline BSNOX & PFI Duration & 1.02 & 9.84 & $\underline{\mathbf{c . 0 0 0 1}}$ & $\underline{\mathbf{0 . 0 0 1}}$ \\
\hline BSNOX & IMAP & -0.520 & -5.00 & $\underline{\mathbf{0 . 0 0 1 5}}$ & $\mathbf{0 . 0 2 3}$ \\
\hline BSNOX & CA50*CA50 & 0.428 & 4.12 & $\underline{\mathbf{0 . 0 0 4 5}}$ & 0.0537 \\
\hline BSNOX & $\begin{array}{c}\text { CA50*PFI } \\
\text { Duration }\end{array}$ & -0.749 & -7.19 & $\underline{\mathbf{s . 0 0 0 1}}$ & $\underline{\mathbf{0 . 0 0 4 2}}$ \\
\hline BSNOX & CA50*IMAT & 0.248 & 2.38 & $\mathbf{0 . 0 3 5 3}$ & 0.3216 \\
\hline BSSoot & CA50 & -2.59 & -8.33 & $\underline{\mathbf{0 . 0 0 0 6}}$ & $\underline{\mathbf{0 . 0 0 4 2}}$ \\
\hline BSSoot & PFI Duration & 0.746 & 2.40 & $\mathbf{0 . 0 3 7 5}$ & 0.266 \\
\hline BTE & CA50 & 0.0141 & 11.2 & $\underline{\mathbf{0 . 0 0 0 2}}$ & $\underline{\mathbf{0 . 0 0 0 5}}$ \\
\hline BTE & IMAP & -0.005 & -3.77 & $\underline{\mathbf{0 . 0 0 7 2}}$ & 0.0769 \\
\hline BTE & PFI Duration & 0.00466 & 3.70 & $\underline{\mathbf{0 . 0 0 8 0}}$ & 0.0813 \\
\hline BSCO & CA50 & 4.74 & 3.80 & $\underline{\mathbf{0 . 0 0 8 4}}$ & 0.0743 \\
\hline BSTHC & PFI Duration & 7.06 & 6.44 & $\underline{\mathbf{0 . 0 0 0 3}}$ & $\underline{\mathbf{0 . 0 0 6 8}}$ \\
\hline $\begin{array}{c}\text { Combustion } \\
\text { Efficiency }\end{array}$ & PFI Duration & -0.0331 & -7.48 & $\underline{\mathbf{0 . 0 0 0 3}}$ & $\underline{\mathbf{0 . 0 0 3 1}}$ \\
\hline $\begin{array}{c}\text { Combustion } \\
\text { Efficiency }\end{array}$ & IMAP & 0.016 & 3.60 & $\underline{\mathbf{0 . 0 0 7 5}}$ & 0.0899 \\
\hline
\end{tabular}

Since CA50 is a variable that is dependent on other parameters, a screening report was generated to determine which of the six parameters of interest may have the largest potential to influence it. This result is presented in Figure 20 and shows PFI duration exhibited the highest contrast and Lenth t-Ratio, as well as the lowest individual p-value of all the six parameters of interest followed by rail pressure. Since DI fueling rate was adjusted to meet load while PFI duration remained constant, another screening approach was taken to consider both DI and PFI effects on CA50 by replacing PFI duration with the percent of total fuel energy input through PFI (percent CNG energy or percentage CNG). The screening report for this approach is displayed in Figure 21 and implied that percentage CNG was very influential on CA50. The very low $p$-values provided confidence in this assumption. 
Screening for CA50 $\left(\mathrm{CA}^{\circ}\right)$

$\triangle$ Contrasts

Term

PFI Duration ( $\mathrm{CA}^{\circ}$ )

Rail Pressure (bar)

DI SOI (CA $\left.{ }^{\circ} B T D C\right)$

Intake Manifold Temperature $\left({ }^{\circ} \mathrm{C}\right)$

Intake Manifold Pressure (psig)

Swirl DC (\%)

PFI Duration $\left(\mathrm{CA}^{\circ}\right)^{\star} \mathrm{PFI}$ Duration $\left(\mathrm{CA}^{\circ}\right)$

PFI Duration (CA $\left.{ }^{\circ}\right)^{\star}$ Rail Pressure (bar)

Rail Pressure (bar) ${ }^{\star}$ Rail Pressure (bar)

PFI Duration (CA $\left.{ }^{\circ}\right)^{\star}$ DI SOI (CA $\left.{ }^{\circ} B T D C\right)$

Rail Pressure (bar) ${ }^{*} \mathrm{DI}$ SOI (CA ${ }^{\circ} \mathrm{BTDC}$ )

DI SOI (CA $\left.{ }^{\circ} B T D C\right) * D I S O I\left(C A{ }^{*} B T D C\right)$

PFI Duration $\left(\mathrm{CA}^{\circ}\right) *$ Intake Manifold Temperature $\left({ }^{\circ} \mathrm{C}\right)$

Rail Pressure (bar) ${ }^{*}$ Intake Manifold Temperature $\left({ }^{\circ} \mathrm{C}\right)$

DI SOI (CA $\left.{ }^{\circ} \mathrm{BTDC}\right){ }^{*}$ Intake Manifold Temperature $\left({ }^{\circ} \mathrm{C}\right)$

Intake Manifold Temperature $\left({ }^{\circ} \mathrm{C}\right){ }^{*}$ Intake Manifold Temperature $\left({ }^{\circ} \mathrm{C}\right)$

PFI Duration ( $\left.\mathrm{CA}^{\circ}\right)^{*}$ Intake Manifold Pressure (psig)

Rail Pressure (bar) ${ }^{\star}$ Intake Manifold Pressure (psig)

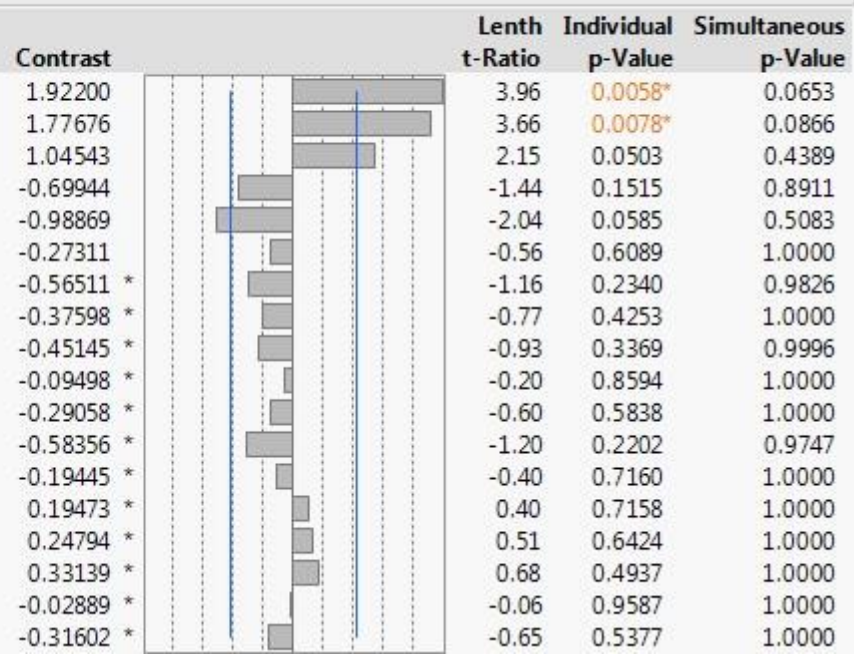

Figure 20: JMP Contrasts Outline for CA50 Screening Report with Six Parameters of Interest

\section{- Screening for CA50 (CA $\left.{ }^{\circ}\right)$}

\section{$\triangle$ Contrasts}

Term

Percent CNG Energy

DI SOI (CA $\left.{ }^{\circ} \mathrm{BTDC}\right)$

Rail Pressure (bar)

Intake Manifold Pressure (psig)

Intake Manifold Temperature $\left({ }^{\circ} \mathrm{C}\right)$

Swirl DC (\%)

Percent CNG Energy ${ }^{*}$ Percent CNG Energy

Percent CNG Energy*DI SOI (CA $\left.{ }^{*} B T D C\right)$

DI SOI (CA $\left.{ }^{\circ} B T D C\right) * D I S O I\left(C A{ }^{*} B T D C\right)$

Percent CNG Energy ${ }^{\star R a i l ~ P r e s s u r e ~(b a r) ~}$

DI SOI (CA $\left.{ }^{\circ} \mathrm{BTDC}\right)^{\star}$ Rail Pressure (bar)

Rail Pressure (bar) ${ }^{\star}$ Rail Pressure (bar)

Percent CNG Energy ${ }^{*}$ Intake Manifold Pressure (psig)

DI SOI (CA ${ }^{\circ} B T D C$ ) Intake Manifold Pressure (psig)

Rail Pressure (bar) Intake Manifold Pressure (psig)

Intake Manifold Pressure (psig) ${ }^{\star}$ Intake Manifold Pressure (psig)

Percent CNG Energy ${ }^{*}$ Intake Manifold Temperature $\left({ }^{\circ} \mathrm{C}\right)$

DI SOI (CA $\left.{ }^{\circ} \mathrm{BTDC}\right){ }^{*}$ Intake Manifold Temperature $\left({ }^{\circ} \mathrm{C}\right)$

\begin{tabular}{l|l|l|l|} 
Contrast & \\
2.58132 \\
1.39764 \\
1.27827 \\
-0.57730 \\
-0.40956 \\
0.07227 \\
$-0.05137 *$ \\
$0.09922 *$ \\
$-0.07010 *$ \\
$-0.09604 *$ \\
$0.25603 *$ \\
$0.14852 *$ \\
$0.13015 *$ \\
$-0.01765 *$ \\
$-0.16266 *$ \\
$0.11622 *$
\end{tabular}

Lenth Individual Simultaneous

t-Ratio p-Value p-Value

$17.63<.0001^{*}<.0001^{*}$

$9.54<.0001^{*} \quad 0.0009^{*}$

$8.73<.0001^{*} \quad 0.0014^{*}$

$\begin{array}{lll}-3.94 & 0.0058^{*} & 0.0663\end{array}$

$\begin{array}{lll}-2.80 & 0.0202^{\star} & 0.2218\end{array}$

$\begin{array}{lll}0.49 & 0.6526 & 1.0000\end{array}$

$\begin{array}{rrr}-0.35 & 0.7490 \quad 1.0000\end{array}$

$\begin{array}{lll}0.68 & 0.4982 & 1.0000\end{array}$

$\begin{array}{rrr}-0.48 & 0.6620 \quad 1.0000\end{array}$

$\begin{array}{lll}-0.66 & 0.5325 & 1.0000\end{array}$

$\begin{array}{lll}1.75 & 0.0904 & 0.6927\end{array}$

$\begin{array}{lll}1.01 & 0.2949 & 0.9979\end{array}$

$\begin{array}{lll}0.89 & 0.3592 & 0.9997\end{array}$

$\begin{array}{lll}-0.12 & 0.9157 & 1.0000\end{array}$

$\begin{array}{lll}-1.11 & 0.2556 & 0.9910\end{array}$

$\begin{array}{lll}0.79 & 0.4148 & 1.0000\end{array}$

$\begin{array}{lll}-0.21 & 0.8460 & 1.0000\end{array}$

$\begin{array}{lll}-0.32 & 0.7682 & 1.0000\end{array}$

Figure 21: JMP Contrasts Outline for CA50 Screening Report with Percentage CNG Substituted for PFI Duration

\subsubsection{Low Load Final Test Matrix development and Verification}

The screening reports generated by JMP offered insight into which of the six parameters of interest were the most influential on $\mathrm{RCCl}$ combustion at the low load operating condition. However, changing the terms used to generate screening reports had profound effects on the outcomes of the reports. Consequently, they must be scrutinized by comparing results with trends that can be seen in the data and considering other parameters, which may have more of an influence or mask the effects of other influences. Through consideration of the screening reports, data from individual tests, literature, and general observations while operating the engine, two parameters were selected to vary while testing the matrix of DI fuels. IMAP was selected due to its perceived influence on BTE and its ease of control. 
IMAP was controlled via the VGT in a stable and repeatable fashion with the Drivven engine controller. Additionally, it does not take significant time for emissions and performance to stabilize after changing the set point compared to other parameters; this allowed test time and fuel consumption to be kept at a minimum. CA50 was selected as the other parameter to be swept. Its effect on emissions and performance is displayed by the screening reports as well as charts of the data. Since this was a dependent parameter, a value of CA50 was targeted by altering the PFI duration. Controlling PFI duration or percentage CNG based on CA50 ensured that combustion phasing did not advance before TDC and produce results inconsistent with the characteristics of RCCl combustion.

A DOE test matrix, presented in Table 12 was constructed with IMAP and CA50 as variables using JMP. The matrix consisted of three levels for both variables, which produced a full factorial matrix of nine discrete test points. Each of these test points was repeated twice forming a test matrix with 27 test runs. A full factorial matrix with two repeats was selected over a fractional factorial matrix to reduce the risk of erroneous trends that might influence conclusions on high reactivity fuel property effects. Set points for the other parameters of interest are presented in Table 13.

Table 12: Final Test Matrix to Evaluate High Reactivity Fuel Property Effects on RCCl Combustion at the Low Load Operating Condition

\begin{tabular}{|c|c|c|c|c|c|}
\hline Test \# & $\begin{array}{l}\text { IMAP } \\
\text { (bar) }\end{array}$ & $\begin{array}{c}\text { CA50 } \\
\text { (CA }{ }^{\circ} \text { ATDC) }\end{array}$ & Test \# & $\begin{array}{l}\text { IMAP } \\
\text { (bar) }\end{array}$ & $\begin{array}{c}\text { CA50 } \\
\text { (CA }{ }^{\circ} \text { ATDC) }\end{array}$ \\
\hline 1 & 1.3 & 4 & 15 & 1.4 & 4 \\
\hline 2 & 1.35 & 4 & 16 & 1.3 & 3 \\
\hline 3 & 1.4 & 5 & 17 & 1.3 & 4 \\
\hline 4 & 1.35 & 3 & 18 & 1.4 & 3 \\
\hline 5 & 1.4 & 4 & 19 & 1.35 & 5 \\
\hline 6 & 1.35 & 5 & 20 & 1.3 & 3 \\
\hline 7 & 1.35 & 3 & 21 & 1.3 & 5 \\
\hline 8 & 1.35 & 4 & 22 & 1.3 & 4 \\
\hline 9 & 1.4 & 3 & 23 & 1.3 & 5 \\
\hline 10 & 1.3 & 5 & 24 & 1.35 & 4 \\
\hline 11 & 1.35 & 5 & 25 & 1.4 & 3 \\
\hline 12 & 1.4 & 5 & 26 & 1.3 & 3 \\
\hline 13 & 1.4 & 4 & 27 & 1.4 & 5 \\
\hline 14 & 1.35 & 3 & & & \\
\hline
\end{tabular}




\section{Table 13: Set points for Other Parameters of Interest for the Low Load Final Test Matrix}

\begin{tabular}{|c|c|}
\hline Parameter & Target Value \\
\hline IMAT & $39^{\circ} \mathrm{C}$ \\
\hline DI SOI* & $50 \mathrm{CA}^{\circ} \mathrm{BTDC}$ \\
\hline Rail Pressure & 500 bar \\
\hline Swirl Valve Position & Fully Open \\
\hline
\end{tabular}

*DI SOI timing for medium and high CN fuels. Lower CN fuels required a DI SOI closer to TDC to prevent misfire.

The CA50 targets in the test matrix, 3, 4, and $5 \mathrm{CA}^{\circ} \mathrm{ATDC}$, comprise a narrow range. This narrow range was selected in effort to ensure that all the high reactivity fuels could be tested at these CA50 values.

Advancing the CA50 past $3 C^{\circ}$ ATDC would result in high in-cylinder PRRs, which proved to be especially prevalent for some of the low $\mathrm{CN}$ high reactivity fuels tested. Retarding the CA50 past $5 \mathrm{CA}^{\circ}$ ATDC could result in misfire if other engine control parameters were not changed. Due to limited quantities of the high reactivity fuels to be tested, expanding the test matrix to include other control parameter changes to allow for a wider range of CA50 targets was not feasible.

A verification of the final test matrix for the low load operating condition was performed using the same locally sourced "pump" diesel and CNG used during the parametric study. A least squares fit was performed using JMP to develop models for $\mathrm{BSNO}_{x}$, BSSoot, and BTE. CA50 and IMAP were initially used as the parameters, but least squares fit analyses were also performed using percentage CNG substituted for CA50. This provided a better fit, based on improved $R^{2}$ and Prob $>F$ ( $p$ values) values for $B S N O_{x}$ and BTE as displayed in Figure 22 and Figure 23. BSSoot did not have a strong correlation with these parameters, but remained very low for all the tests. 


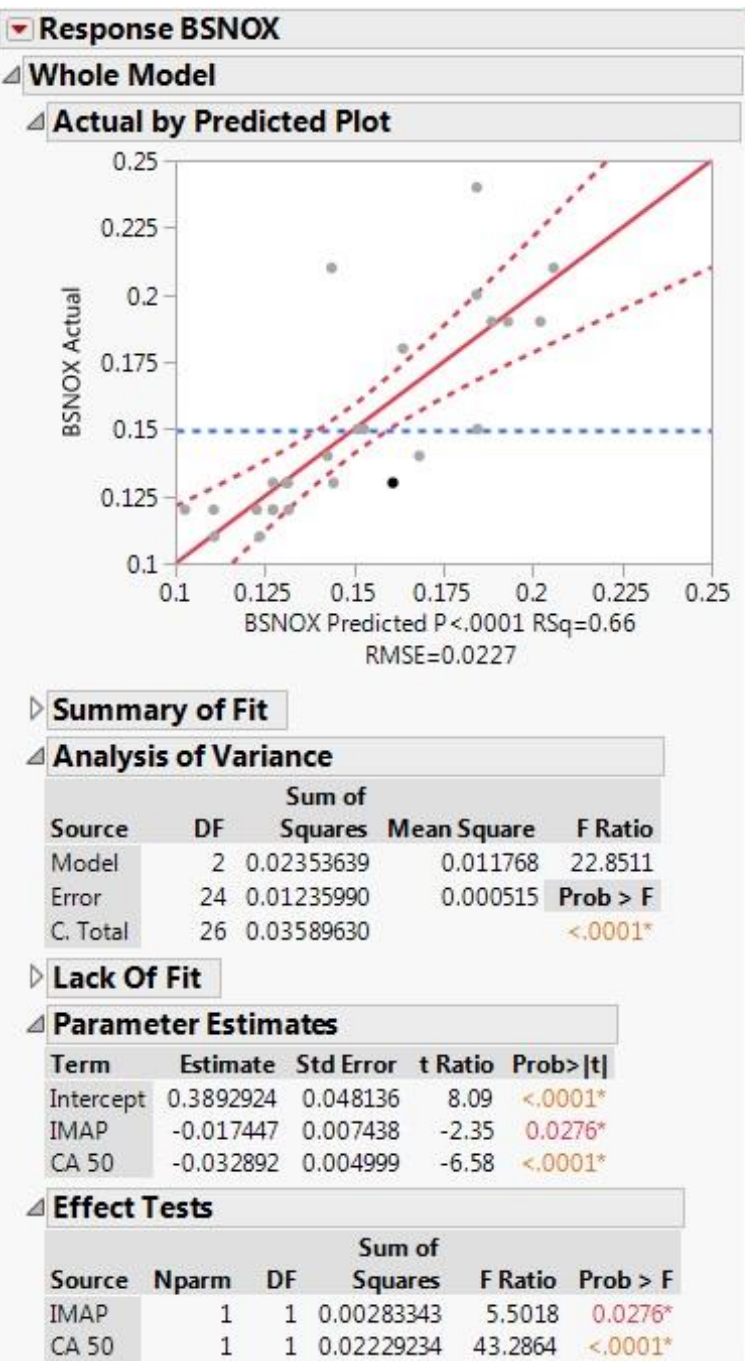

- Response BSNOX

$\triangle$ Whole Model

$\triangle$ Actual by Predicted Plot

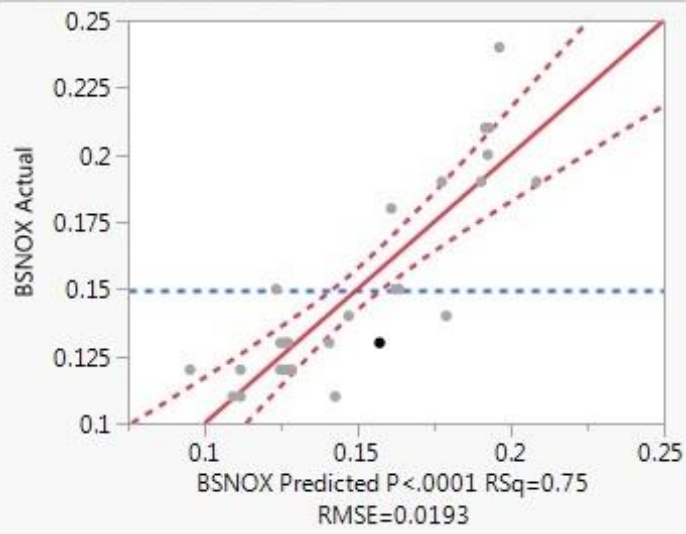

$\triangle$ Summary of Fit

$\triangle$ Analysis of Variance

\begin{tabular}{|c|c|c|c|c|c|c|}
\hline Source & \multicolumn{3}{|c|}{$\begin{array}{l}\text { Sum of } \\
\text { Squares }\end{array}$} & \multicolumn{2}{|c|}{ Mean Square } & F Ratio \\
\hline Model & 2 & \multicolumn{2}{|c|}{0.02698827} & \multicolumn{2}{|c|}{0.013494} & 36.3559 \\
\hline Error & 24 & \multicolumn{2}{|c|}{0.00890802} & \multicolumn{2}{|r|}{0.000371} & Prob $>F$ \\
\hline C. Total & 26 & \multicolumn{2}{|c|}{0.03589630} & & & $<, 0001^{*}$ \\
\hline \multicolumn{6}{|l|}{ Lack Of Fit } & \\
\hline \multicolumn{7}{|c|}{ Parameter Estimates } \\
\hline Term & \multicolumn{2}{|c|}{ Estimate } & Std & Error & t Ratio & Prob $>|t|$ \\
\hline Intercept & \multirow{2}{*}{\multicolumn{2}{|c|}{$\begin{array}{l}1.1902005 \\
0.0263851\end{array}$}} & 0.12 & 22321 & 9.73 & $<, 0001^{*}$ \\
\hline IMAP & & & 0.00 & 07742 & 3.41 & $0.0023^{*}$ \\
\hline Percent CNG & \multicolumn{2}{|c|}{-1.673428} & 0.20 & 00933 & -8.33 & $<, 0001^{*}$ \\
\hline
\end{tabular}

$\triangle$ Effect Tests

\begin{tabular}{|c|c|c|c|c|c|}
\hline Source & Nparm & DF & $\begin{array}{l}\text { Sum of } \\
\text { Squares }\end{array}$ & F Ratio & Prob $>$ F \\
\hline IMAP & 1 & 1 & 0.00431056 & 11.6135 & $0.0023^{*}$ \\
\hline Percent CNG & 1 & 1 & 0.02574422 & 69.3601 & $<, 0001^{*}$ \\
\hline
\end{tabular}

Figure 22: JMP Least Squares Fit Model of BSNOX with IMAP and CA50 versus IMAP and Percentage CNG 

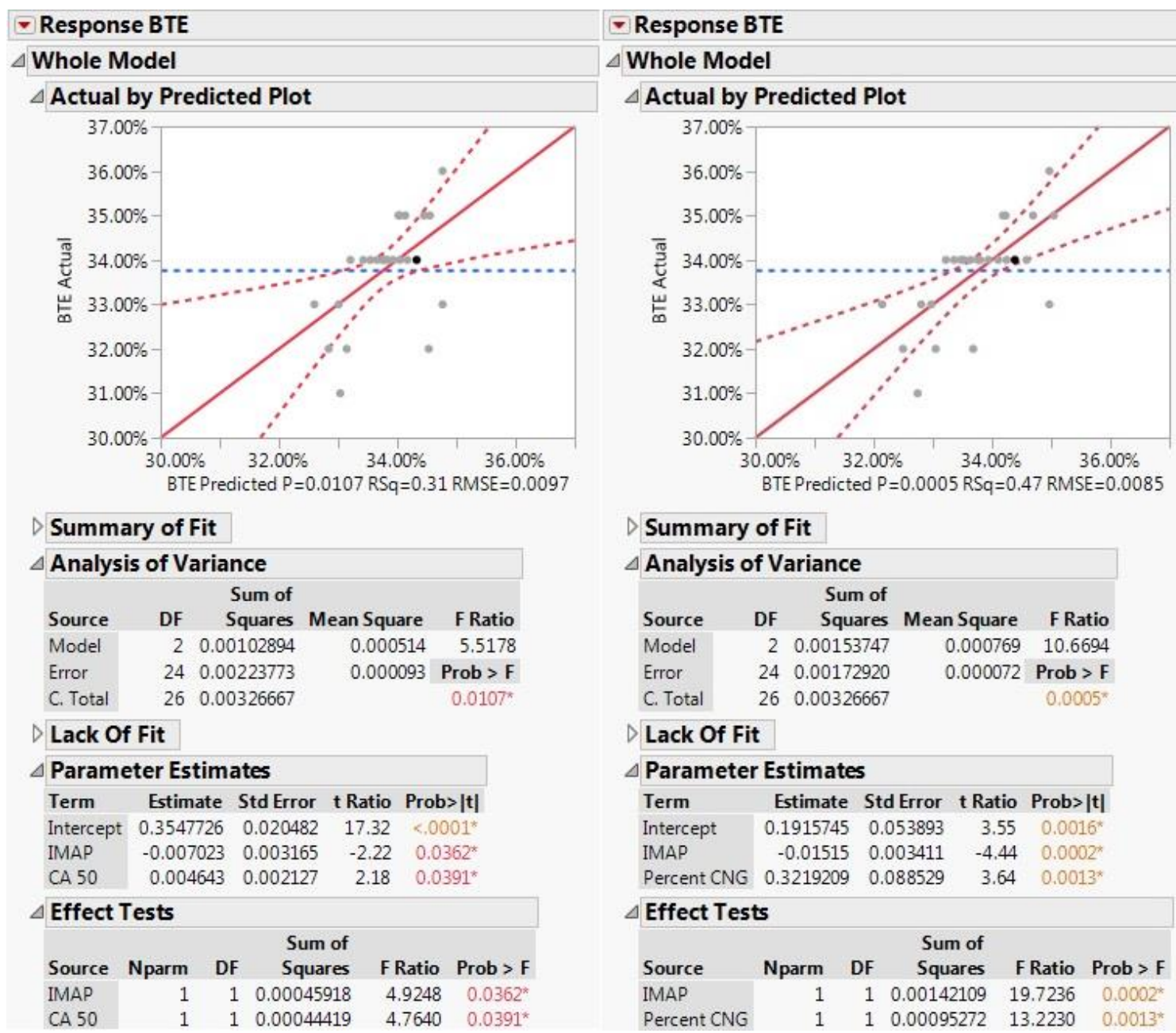

$\triangleright$ Summary of Fit

$\triangle$ Analysis of Variance

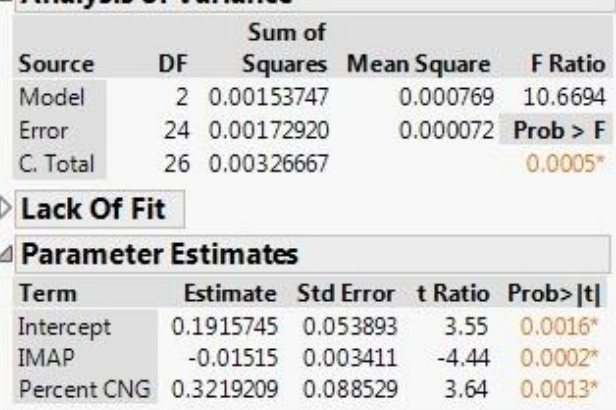

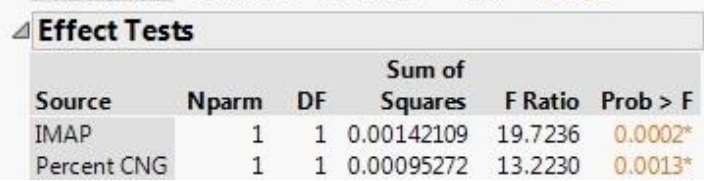

Figure 23: JMP Least Squares Fit Model of BTE with IMAP and CA50 versus IMAP and Percentage CNG

It was concluded from Figure 22 and Figure 23 that IMAP and percentage CNG (adjusted to reach a CA50 target) were influential on $\mathrm{BSNO}_{x}$ emissions and BTE. Based on the parameter estimates of the model, $\mathrm{BSNO}_{x}$ emissions decreased and BTE increased as IMAP decreased and percentage CNG increased. Table 14 presents the average, maximum and minimum values for the entire test matrix. BSNOx emissions were lower, on average, than the federal standard for 2010 and later heavy-duty engines ( $0.2 \mathrm{~g} / \mathrm{bhp}$-hr), albeit at a single steady state load point. Using soot emissions as a surrogate for PM, BSSoot emission were also lower than the federal standard for PM emissions for heavy-duty engines (10 mg/bhp-hr). BTE was high considering the low load imposed on the engine and compared to diesel only operation (27.5\%). These observations confirmed that RCCI was being achieved, while the statistical results obtained from JMP provided the basis for moving forward with the final low load test matrix to quantify the effects of high reactivity fuel properties on $\mathrm{RCCl}$. 
Table 14: Emissions and Performance Averages, Maximums and Minimums for Pump Diesel and CNG from the Low Load Final Test Matrix

\begin{tabular}{|c|c|c|c|c|c|c|}
\hline & $\begin{array}{c}\text { BSCO } \\
\text { (g/bhp-hr) }\end{array}$ & $\begin{array}{c}\text { BSNOX } \\
\text { (g/bhp-hr) }\end{array}$ & $\begin{array}{c}\text { BSTHC } \\
\text { (g/bhp-hr) }\end{array}$ & $\begin{array}{c}\text { BSSoot } \\
\text { (mg/bhp-hr) }\end{array}$ & BTE (\%) & $\begin{array}{c}\text { Combustion } \\
\text { Efficiency (\%) }\end{array}$ \\
\hline Average & 22.44 & 0.15 & 25.19 & 2.45 & $34 \%$ & $84 \%$ \\
\hline Maximum & 27.01 & 0.24 & 32.41 & 3.75 & $36 \%$ & $88 \%$ \\
\hline Minimum & 16.08 & 0.11 & 18.51 & 1.40 & $31 \%$ & $81 \%$ \\
\hline
\end{tabular}

\subsubsection{High Load Operating Condition Parametric Study}

Preliminary tests were performed to determine the maximum load at 2100 RPM that could be achieved during $\mathrm{RCCl}$ operation for the high load operating condition. These tests were performed using locally sourced CNG and the Cert fuel since large quantities were available and its CN, 45.6, was relatively close to the average $\mathrm{CN}$ of all the fuels, 42.5. The load that was ultimately selected for the high load operating condition was $67 \mathrm{ft}$-lb or approximately 6 bar BMEP. Depending on engine control parameters, higher loads could be achieved with safe in-cylinder PRRs using CNG and the Cert fuel, but it was determined that 6 bar BMEP would likely be the highest load achievable for some of the high reactivity fuels. Additionally, as previously mentioned, Curran, et al. found 6 bar BMEP to be the maximum achievable load based on safe in-cylinder PRRs for RCCl operation at similar operating conditions [8]. Although 6 bar BMEP is not necessarily considered a high load, for ease of reference this load will be termed the high load operating condition herein.

Based on knowledge gained from the low load parametric study and through preliminary activities performed at the high load operating point, control parameters were selected for the DOE parametric study matrix. At this operating condition it was determined that the introduction of EGR actually increased the maximum in-cylinder PRR. This was attributed to a rise in IMAT resulting from the hot exhaust gases introduced into the intake manifold, which in turn advanced combustion phasing. Thus, EGR was not used at this operating condition. Although it was demonstrated in the low load parametric study, and has also been documented in RCCl literature that IMAT affects the RCCl combustion process, a single IMAT was used for the high load operating condition due to limitations of the control system. After changing the IMAT set point, a modest amount of time was required to achieve stabilization. Considering that there was limited high reactivity fuel available for testing, it was decided that this stabilization time would result in the use of too much fuel and thus a constant IMAT of $39^{\circ} \mathrm{C}$ was targeted for all high load tests. Several DI rail pressures were experimented with during these preliminary activities. It was observed that using a rail pressure greater than 400 bar during RCCI operation caused the engine torque output to become very cyclic and unstable. It was possible that adjusting the throttle controller delay or dynamometer speed controller PID coefficients may have reduced the torque fluctuations. However, since this problem was not apparent at the low load operating condition or for CDC, It was suspected that rail pressures higher than 400 bar combined with early DI SOI timing resulted in the high reactivity fuel impinging on the cylinder walls, which caused the $\mathrm{RCCl}$ combustion to become very unrepeatable from cycle-to-cycle. As such, a rail pressure of 400 bar was used throughout the high load testing.

Although a single DI event was used for the low load $\mathrm{RCCl}$ operation, it was determined through the preliminary activities that a pilot injection event, in addition to the main DI event, was beneficial for the 
high load operating condition to reduce $\mathrm{NO}_{\mathrm{x}}$ emissions and in-cylinder PRR. The duration and timing of this pilot injection event was varied for the parametric study test matrix. In addition to the pilot injection parameters, CA50 controlled by PFI duration, main DI SOI timing, and IMAP were selected as the other variables to use in the high load parametric study matrix as displayed in Table 15 . Relative values for these parameters were determined by preliminary activities focusing on cycle-to-cycle stability of the engine and safe in-cylinder PRR. The test matrix developed with the use of JMP software is presented in Table 16.

Table 15: Input Variable Levels and Values for the High Load Parametric Study

\begin{tabular}{|c|c|c|}
\hline Input Variable & Levels & Values \\
\hline CA50 (CA $^{\circ}$ ATDC) & 3 & $3,4,5$ \\
\hline Number of Injections & 2 & 2 \\
\hline Main DI Start of Injection (CA ${ }^{\circ}$ BTDC) & 2 & 60,65 \\
\hline Pilot Injection Amount (\% of Main DI) & 3 & $5,10,20$ \\
\hline Pilot Injection Advance from Main DI (ms) & 2 & $1.3,1.6$ \\
\hline IMAP (bar) & 2 & $1.4,1.5$ \\
\hline
\end{tabular}

Table 16: L18 Chakravarty DOE Test Matrix for the High Load Parametric Study

\begin{tabular}{|c|c|c|c|c|c|}
\hline Test \# & $\begin{array}{c}\text { CA50 (CA } \\
{ }^{\circ} \text { ATDC) }\end{array}$ & $\begin{array}{c}\text { Pilot (\% } \\
\text { Main DI) }\end{array}$ & $\begin{array}{c}\text { Pilot } \\
\text { Advance } \\
\text { (ms) }\end{array}$ & $\begin{array}{c}\text { Main DI } \\
\text { SOI (CA } \\
{ }^{\circ} \text { BTDC) }\end{array}$ & $\begin{array}{c}\text { IMAP } \\
\text { (bar) }\end{array}$ \\
\hline 1 & 3 & 10 & 1.6 & 65 & 1.4 \\
\hline 2 & 5 & 20 & 1.3 & 60 & 1.4 \\
\hline 3 & 4 & 10 & 1.3 & 60 & 1.4 \\
\hline 4 & 3 & 20 & 1.3 & 65 & 1.5 \\
\hline 5 & 3 & 5 & 1.6 & 65 & 1.4 \\
\hline 6 & 3 & 5 & 1.3 & 60 & 1.4 \\
\hline 7 & 5 & 20 & 1.6 & 65 & 1.4 \\
\hline 8 & 4 & 20 & 1.3 & 65 & 1.5 \\
\hline 9 & 5 & 5 & 1.3 & 65 & 1.5 \\
\hline 10 & 4 & 5 & 1.6 & 60 & 1.5 \\
\hline 11 & 4 & 10 & 1.6 & 65 & 1.4 \\
\hline 12 & 3 & 20 & 1.6 & 60 & 1.5 \\
\hline 13 & 4 & 20 & 1.6 & 65 & 1.4 \\
\hline 14 & 5 & 5 & 1.6 & 65 & 1.4 \\
\hline 15 & 5 & 10 & 1.6 & 60 & 1.5 \\
\hline 16 & 5 & 10 & 1.3 & 65 & 1.5 \\
\hline 17 & 4 & 5 & 1.3 & 65 & 1.5 \\
\hline 18 & 3 & 10 & 1.3 & 65 & 1.5 \\
\hline
\end{tabular}

Similar to the approach for the low load RCCI parametric study, a L18 Chakravarty DOE test matrix was used. For each test, the IMAP, main DI SOI timing, pilot injection percentage, and pilot injection timing 
in milliseconds from the main DI SOI timing were set, then the desired CA50 was targeted by adjusting the PFI duration. This approach was used instead of specific PFI durations based on the results of the low load parametric study.

Once the L18 Chakravarty DOE test matrix was completed for the high load operating condition, the data was fed into JMP for analysis. Similar to the low load parametric study, a screening analysis was applied to the collected data. However, for the high load parametric study FCE was included in the screening analysis in addition to BTE. It was observed through the low load testing of the high reactivity fuels, that FCE was a better metric to define efficiency rather than BTE because of the inclusion of combustion efficiency in the denominator of the equation for BTE. A low combustion efficiency could inflate BTE, which will be discussed further in subsequent sections. Additionally, maximum in-cylinder PRR was also included in the screening analysis because of the limitation it imposes on the maximum achievable engine load during $\mathrm{RCCl}$ operation. The first screening analysis performed used only the control parameters listed in Table 16. The results of this analysis are presented in Table 17. 
Table 17: Potentially Influential Parameters from JMP Screening Report Contrasts Outline

\begin{tabular}{|c|c|c|c|c|c|}
\hline Effect & Parameter & Contrast & $\begin{array}{l}\text { Lenth } \\
\text { t-Ratio }\end{array}$ & $\begin{array}{l}\text { Individual } \\
\text { p-value }\end{array}$ & $\begin{array}{c}\text { Simultaneous } \\
\text { p-value }\end{array}$ \\
\hline BSNOX & \multicolumn{5}{|c|}{ No parameters with $p$-values less than .05} \\
\hline BSSoot & Main DI SOI & 0.535 & 2.45 & 0.0301 & 0.281 \\
\hline BTE & Main DI SOI & -0.005 & -5.07 & $\underline{0.0032}$ & 0.0229 \\
\hline BTE & CA50 & 0.005 & 4.68 & 0.0041 & 0.0309 \\
\hline BTE & Pilot Advance & -0.004 & -4.06 & 0.0066 & 0.0565 \\
\hline BTE & $\begin{array}{l}\text { Main DI SOI*Pilot } \\
\text { Advance }\end{array}$ & -0.005 & -4.36 & $\underline{0.006}$ & 0.0435 \\
\hline BTE & Pilot Advance*Pilot\% & -0.002 & -2.29 & 0.0407 & 0.358 \\
\hline $\begin{array}{l}\text { Combustion } \\
\text { Efficiency }\end{array}$ & CA50 & -0.015 & -6.33 & $\underline{0.0007}$ & $\underline{0.0082}$ \\
\hline FCE & Main DI SOI & -0.005 & -2.94 & 0.0182 & 0.185 \\
\hline FCE & Pilot Advance & -0.0040 & -2.39 & 0.0331 & 0.300 \\
\hline FCE & Main SOI*Pilot Advance & -0.004 & -2.35 & 0.0342 & 0.319 \\
\hline $\mathrm{BSCO}$ & CA50 & 0.779 & 8.28 & $\underline{0.0005}$ & $\underline{0.0027}$ \\
\hline $\mathrm{BSCO}$ & Main DI SOI & 0.350 & 3.72 & $\underline{0.007}$ & 0.0774 \\
\hline $\mathrm{BSCO}$ & IMAP & 0.291 & 3.09 & 0.0149 & 0.158 \\
\hline $\mathrm{BSCO}$ & Pilot Advance & 0.247 & 2.63 & 0.0241 & 0.25 \\
\hline $\mathrm{BSCO}$ & CA50*CA50 & -0.249 & -2.65 & 0.024 & 0.248 \\
\hline BSTHC & CA50 & 2.01 & 5.91 & $\underline{0.0012}$ & 0.0129 \\
\hline BSTHC & CA50*CA50 & -0.728 & -2.15 & 0.048 & 0.418 \\
\hline BSTHC & Pilot \%*Pilot Advance & 0.830 & 2.45 & 0.0303 & 0.277 \\
\hline Max PRR & CA50 & -91.6 & -8.44 & $\underline{0.0002}$ & $\underline{0.0016}$ \\
\hline Max PRR & Main DI SOI & 24.4 & 2.25 & 0.039 & 0.376 \\
\hline
\end{tabular}

Identical to the low load parametric study, parameters that had individual $p$-values less than 0.05 are presented as potentially influential on $\mathrm{RCCl}$ at the high load operating condition. Notably, none of the parameters included in this screening analysis exhibited a p-value less than 0.05 for $\mathrm{BSNO}_{\mathrm{x}}$. With respect to the other effects, CA50 and main DI SOI appeared to have a dominant influence. For each of the effects presented, CA50 or main DI SOI exhibited the highest contrast compared to the other potentially influential parameters. Since CA50 targets were achieved by increasing or decreasing the PFI duration and subsequently percentage CNG, another analysis was performed including percentage CNG. The results from this analysis are presented in Table 18. 
Table 18: Potentially Influential Parameters from JMP Screening Report Contrasts Outline including Percentage CNG

\begin{tabular}{|c|c|c|c|c|c|}
\hline Effect & Parameter & Contrast & $\begin{array}{l}\text { Lenth } \\
\text { t-Ratio }\end{array}$ & $\begin{array}{c}\text { Individual } \\
\text { p-value }\end{array}$ & $\begin{array}{c}\text { Simultaneous } \\
\text { p-value }\end{array}$ \\
\hline BSNOX & Percentage CNG & -0.207 & -3.18 & 0.0137 & 0.142 \\
\hline BSSoot & Percentage CNG & -0.767 & -6.7 & $\underline{0.0008}$ & 0.0073 \\
\hline BSSoot & Pilot \% & 0.265 & 2.31 & 0.0371 & 0.337 \\
\hline BSSoot & Percentage CNG*CA50 & 0.323 & 2.82 & 0.0209 & 0.208 \\
\hline BTE & Percentage CNG & 0.009 & 6.8 & $\underline{0.0008}$ & $\underline{0.0062}$ \\
\hline BTE & IMAP & -0.003 & -2.46 & 0.0294 & 0.275 \\
\hline $\begin{array}{l}\text { Combustion } \\
\text { Efficiency }\end{array}$ & CA50 & -0.015 & -5.49 & $\underline{0.001}$ & 0.0163 \\
\hline FCE & Main DI SOI & -0.005 & -2.75 & 0.0232 & 0.222 \\
\hline FCE & Pilot Advance & -0.004 & -2.24 & 0.0436 & 0.376 \\
\hline FCE & $\begin{array}{l}\text { Main DI SOI*Pilot } \\
\text { Advance }\end{array}$ & -0.004 & -2.16 & 0.0493 & 0.416 \\
\hline $\mathrm{BSCO}$ & CA50 & 0.779 & 5.37 & $\underline{0.0015}$ & 0.0164 \\
\hline $\mathrm{BSCO}$ & Main DI SOI & 0.35 & 2.41 & 0.0344 & 0.293 \\
\hline BSTHC & CA50 & 2.01 & 4.20 & $\underline{0.0056}$ & 0.0521 \\
\hline Max PRR & CA50 & -91.6 & -8.18 & 0.0002 & $\underline{0.0025}$ \\
\hline Max PRR & Main DI SOI & 24.4 & 2.18 & 0.0456 & 0.410 \\
\hline
\end{tabular}

Including percentage CNG in the analysis does show a potential effect on $\mathrm{BSNO}_{\mathrm{x}}$ emissions, although the confidence in its influence was not as high as it was for the other effects. Nonetheless, this analysis provided additional confidence in the conclusion that main DI SOI and CA50, or percentage CNG as the control parameter for CA50, were the most influential parameters on $\mathrm{RCCl}$ performance at this operating condition.

\subsubsection{High Load Final Test Matrix development}

From the screening analysis results, CA50 and main DI SOI were chosen as the variables to sweep during the testing of the high reactivity fuels. Similar to the low load test matrix, a full factorial design was used with two repeats. Three levels of CA50, 3, 4 and 5 crank angle degrees ATDC, were selected as targets in the high load final test matrix. Similar to the low load operating condition, this narrow range of CA50 targets was selected in an effort to ensure that all the fuels could be operated at these CA50 targets and to limit the size of the test matrix to ensure there was enough high reactivity test fuel to complete the experiment. Only two set points were selected for main DI SOI because there was generally a limited range of main DI SOI timings that the engine would operate at for a particular high reactivity fuel.

Additionally, this allowed for a test matrix of 18 data collections, versus 27 for the low load operating condition, which was desirable based on the amount of high reactivity fuel that remained and the fact that the high load operating condition would consume fuel at a faster rate than the low load operating condition. The final test matrix developed with JMP is presented in Table 19. 
Table 19: Final Test Matrix to Evaluate High Reactivity Fuel Property Effects on RCCl Combustion at the High Load Operating Condition

\begin{tabular}{|c|c|c|c|c|c|}
\hline Test \# & $\begin{array}{c}\text { CA50 } \\
\text { (CA }{ }^{\circ} \text { ATDC) }\end{array}$ & $\begin{array}{l}\text { Main DI SOI } \\
{\text { (CA }{ }^{\circ} \text { BTDC)* }}^{*}\end{array}$ & Test \# & $\begin{array}{c}\text { CA50 } \\
\left.\text { (CA }{ }^{\circ} A T D C\right)\end{array}$ & $\begin{array}{l}\text { Main DI SOI } \\
{\text { (CA }{ }^{\circ} \text { BTDC)* }}^{*}\end{array}$ \\
\hline 1 & 3 & 65 & 10 & 5 & 60 \\
\hline 2 & 5 & 65 & 11 & 4 & 60 \\
\hline 3 & 3 & 60 & 12 & 3 & 60 \\
\hline 4 & 3 & 65 & 13 & 5 & 65 \\
\hline 5 & 4 & 65 & 14 & 5 & 60 \\
\hline 6 & 3 & 65 & 15 & 4 & 60 \\
\hline 7 & 4 & 65 & 16 & 4 & 60 \\
\hline 8 & 4 & 65 & 17 & 5 & 65 \\
\hline 9 & 3 & 60 & 18 & 5 & 60 \\
\hline
\end{tabular}

* Main DI SOI timing for medium and high CN fuels. Lower CN fuels required main DI SOI closer to TDC to prevent misfire.

As noted, the main DI SOI timing presented in Table 19 was used for the medium and high $\mathrm{CN}$ number fuels, while the low $\mathrm{CN}$ fuels used $50^{\circ} \mathrm{BTDC}$ and $45^{\circ} \mathrm{BTDC}$. Advancing the main DI SOI timing further for the low CN fuels resulted in misfire. Regardless, FACE 4, which had the lowest CN, could only be tested at a CA50 of approximately $5{ }^{\circ}$ ATDC because of excessive in-cylinder PRRs. Thus, data only exists for six tests points for FACE 4. Settings for the other parameters of interest are presented in Table 20.

Table 20: Set points for Other Parameters of Interest for the High Load Final Test Matrix

\begin{tabular}{|c|c|}
\hline Parameter & Target Value \\
\hline IMAT & $39{ }^{\circ} \mathrm{C}$ \\
\hline Pilot Injection Amount (\% of Main DI) & $10 \%$ \\
\hline Pilot Injection Advance from Main DI & $1.3 \mathrm{~ms}$ \\
\hline Rail Pressure & $400 \mathrm{bar}$ \\
\hline Swirl Valve Position & Fully Open \\
\hline
\end{tabular}




\section{Experimental Results and Analysis}

Comparing engine operation on different fuels to determine the effects of fuel properties on combustion, exhaust emissions and overall performance parameters is a complex task. Given the complex nature of ICEs, especially modern engines with advanced technology such as common rail fuel injection, EGR, and VGT, it can be difficult to determine if, for example, a change in a particular emissions measurement was related to fuel property effects or variability found in the engine's hardware and control algorithms, or laboratory equipment. Further complicating analyses, certain fuel properties, such as $\mathrm{CN}$, may have such a strong effect that they mask other properties and as noted in Section 4.7, several of these properties correlate with each other. Additionally, when comparing fuels that retain significantly different properties, engine control parameters such as the DI SOI timing, may need to be changed to exploit the benefits of the fuel or simply to obtain stable combustion. To help mitigate these concerns, the parametric studies presented in Section 5.3 were performed. These studies helped to determine the parameters that had a crucial influence on the regulated exhaust emissions of NOx, PM (soot as a surrogate), THC, and CO and engine performance during RCCI operation. Based on these results, test matrices were developed that varied IMAP for the low load operating condition and main DI SOI timing for the high load operating condition. Additionally, CA50 was controlled for both low and high load operating conditions rather than target specific percentage CNG values. CA50 is a common metric used to ensure combustion phasing is similar when comparing fuels and technologies.

Data analysis was performed with the aid of JMP statistical software. Similar to the parametric studies detailed in Section 5.3, screening analyses were performed on performance values, exhaust emissions measurements, and efficiency calculations with fuel properties as the variables. CN, AC, T90, T10 and SG were used in the screening analysis. Hydrogen-to-carbon ratio and the net heat of combustion were not included since these values were not explicitly known for all fuels. However, hydrogen-to-carbon ratio correlates well with SG ( $R^{2}$ of 0.82 ) and net heat of combustion correlates well with $A C\left(R^{2}\right.$ of 0.95$)$, therefore if SG or AC is deemed influential, it can be inferred that hydrogen-to-carbon ratio or net heat of combustion, respectively, is influential. Results from the screening reports were then used to fit models to the data via a least squares fit approach. These models were not intended to be used for simulations or empirical relations, but to expose and discern high reactivity fuel property effects on $\mathrm{RCCl}$. Fuel property effects and trends identified in the screening analysis and least squares fit models were then further scrutinized based on statistics provided by JMP and visually through plots of the data. Box and whisker plots created with Matlab were the primary plots used to display the data. These plots allowed for multiple data points to be plotted concisely for each fuel on the same plot while providing statistical information about the data. The horizontal lines for each data segment represent the median of the data for each fuel, the upper and lower edges of the box are the $75^{\text {th }}$ and $25^{\text {th }}$ percentiles of the data (interquartile range), respectively, the whiskers represent one and a half times the interquartile range, and small circles indicate outliers. Of particular interest in the box and whisker plots are the median values and the width of the box (either interquatile range or whisker range). The median value provides a relative change in the values from one property to another and the width provides the influence of the parameters on the overall effect. When examining effects to include or discard in the least squares fit models, their effect on the $\mathrm{R}^{2}$ value and low $\mathrm{p}$-values were primary considerations. All the effects included in the models presented below had a p-value less than 0.0001 unless otherwise noted. 
Note that the data presented in the box and whisker plots includes all the data collected for each high reactivity fuel at the load condition specified. For the low load operating condition, this includes all the data points described in Table 12. IMAP and CA50 (controlled by adjusting the percentage CNG) were varied for the low load operating condition while the other engine control parameters were fixed. Table 19 details the set points for Main DI SOI timing and CA50, which were varied for the high load operating condition. Note that deviations in the DI SOI timing had to be made for the low $\mathrm{CN}$ fuels at both load conditions to avoid misfire. This is detailed in Sections 5.3.2 and 5.3.4, and noted in subsequent sections when pertinent. Additionally, due to the wide $\mathrm{CN}$ range of the high reactivity fuels, the medium and high CN fuels could not be tested at the SOI timing that the low CN fuels were tested at. Doing so would have resulted in excessive in-cylinder PRRs for $\mathrm{RCCl}$ operation with the medium and high $\mathrm{CN}$ fuels.

\subsection{Ratio of Low Reactivity to High Reactivity Fuel}

The ratio of low reactivity fuel to high reactivity fuel is often referred to as substitution ratio, PFI fraction, premixed fuel ratio, or as preferred for this research, percentage CNG. Percentage CNG, as used in this document, is defined as the percentage of total fuel energy input into the cylinder as CNG. A correlation between $\mathrm{CN}$ and percentage CNG was found for the low and high load operating conditions. As the $\mathrm{CN}$ of the high reactivity fuel, or diesel in this instance, was increased the percentage CNG must also be increased to achieve the combustion phasing targets as dictated by the test matrices (CA50 of 3,4 , or $5^{\circ} \mathrm{CA}$ ATDC). The increase in percentage CNG was necessary because of the decreased ignition delay offered by higher CN fuels. Increasing the percentage CNG effectively increased the ignition delay of the fuel mixture because of the high octane rating of CNG. Figure 24 displays the percentage CNG for all the tests performed for each high reactivity fuel at the low load operating condition.

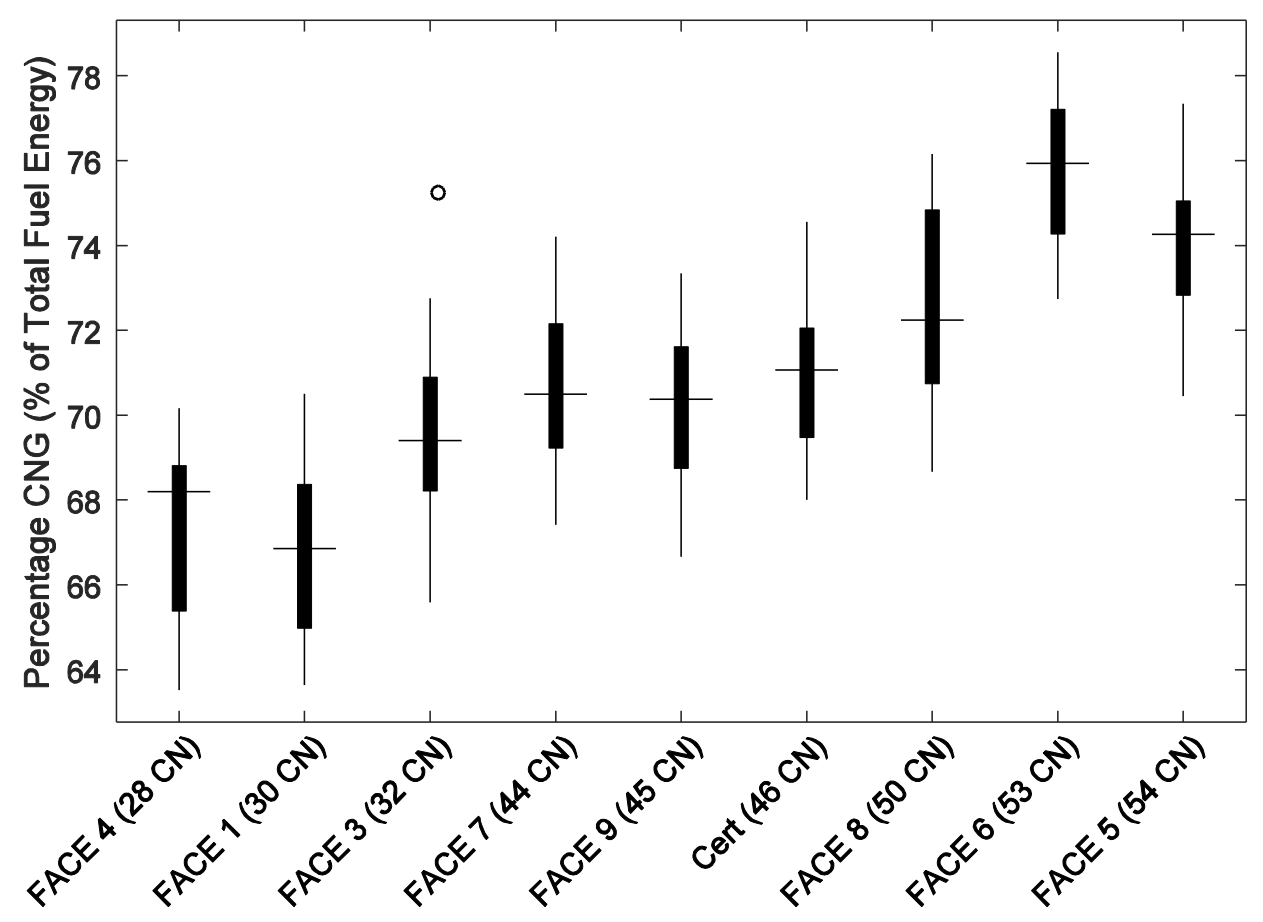

Figure 24: Percentage CNG for the Low Load Operating Condition (Varying IMAP and CA50) 
A least squares fit model generated with JMP further confirmed that $\mathrm{CN}$ was influential on percentage CNG with a $p$-value less than 0.0001 . Engine control parameters and test matrix targets that changed among the data, including CA50, IMAP, and DI SOI timing were also deemed significant and were included in the model. Additionally, the model included the product of $\mathrm{CN}$ and $\mathrm{AC}$ terms that was deemed influential with a high level of confidence based on a $p$-value less than 0.0001 . The model is defined by Equation 37.

\section{Equation 37: Least Squares Fit Model for Percentage CNG at the Low Load Operating Condition}

$$
\begin{aligned}
& \text { Percentage CNG } \\
& \qquad \begin{aligned}
& 53.765+1.7079 * C A 50+0.42788 * C N+1.2202 * I M A P-0.3 .1731 * D I \text { SOI } \\
& -6.3008 * 10^{-3} *(C N-43.003) *(A C-32.762)
\end{aligned}
\end{aligned}
$$

The model exhibited an $R^{2}$ value of 0.84 . Percentage $C N G$ was adjusted to achieve the CA50 targets of the test matrix. From the model, it can be observed that to retard the CA50 higher percentage CNG is required and vice versa. Increasing the IMAP resulted in a higher percentage CNG, while advancing the DI SOI timing resulted in a lower percentage CNG. With regards to $A C$, its association with $C N$ indicates that a trend of higher percentage CNG with lower AC exists for fuels with a CN greater than 43 (medium and high CN fuels). The trend reverses for low CN fuels, where a lower percentage CNG was required for proper combustion phasing for fuels with low AC. These trends can be observed in Figure 24; FACE 6 and FACE 5, both low AC fuels required more percentage CNG on average than FACE 8, which was the other high CN fuel. Additionally, the Cert fuel, which had a very low AC content, required more percentage CNG on average than the other medium CN fuels. The deviation from this trend for the low $\mathrm{CN}$ fuels may be related to the retarded DI SOI timing that had to be used for those fuels to prevent misfire. 


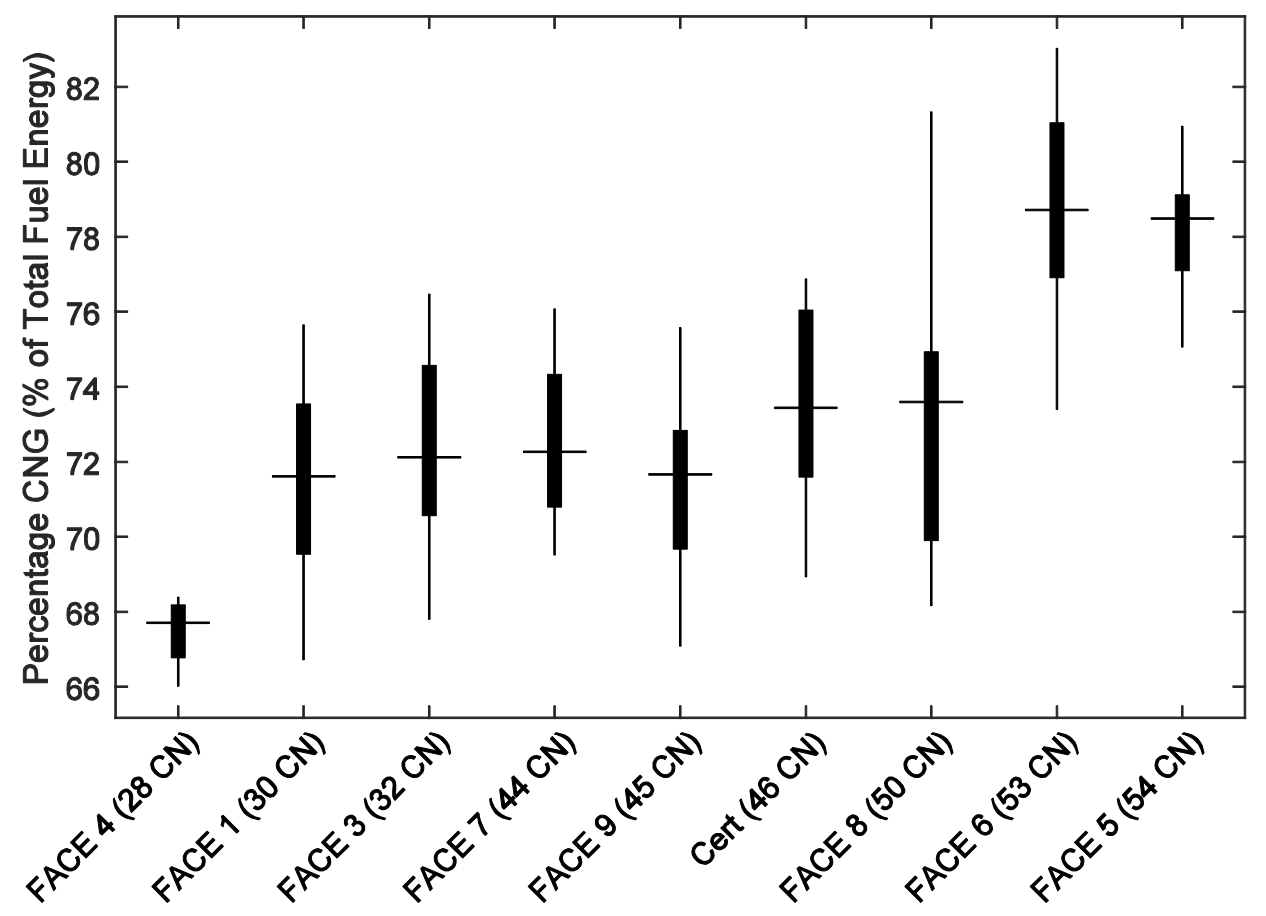

Figure 25: Percentage CNG for the High Load Operating Condition (Varying Main DI SOI Timing and CA50)

Results similar to the low load operating condition were observed the high load operating condition as shown in Figure 25. Comparing the percentage CNG for fuels at each end of the CN spectrum certainly provides an indication of the influence of $\mathrm{CN}$. A least squares fit model developed with JMP, defined by Equation 38, also indicated that the main DI SOI timing, CA50 and AC and were influential, with a high level of confidence, on percentage CNG at the high load operating condition.

\section{Equation 38: Least Squares Fit Model for Percentage CNG at the High Load Operating Condition}

Percentage $C N G$

$$
\begin{aligned}
& =78.638+0.70181 * C N-0.65237 * \text { main DI SOI }+0.96535 * C A 50-0.060363 \\
& * A C
\end{aligned}
$$

The $\mathrm{R}^{2}$ value for Equation 38 was 0.83 . Similar to the low load operating condition, advancing the main $\mathrm{DI}$ event reduced the percentage CNG necessary to meet the CA50 target. Additionally, to achieve further retarded CA50 targets, a higher percentage CNG was necessary. Visually inspecting Figure 25 for the AC trend reveals similar observations when compared to the low load data. Again, FACE 6 and FACE 5 required noticeably higher percentage of CNG than FACE 8. The Cert fuel also required higher percentage CNG on average than the other medium CN fuels. However, an investigation of the trend for the low $\mathrm{CN}$ high reactivity fuels was inconclusive. FACE 1, a low AC fuel required similar percentage CNG compared to FACE 3 (high AC) and limited tests were performed for FACE 4 because of excessive incylinder PRRs.

Based on the trends observed in Figure 24 and Figure 25, as well as the trends quantified with least squares fit models, CN and AC were influential on percentage CNG at the low and high load operating 
conditions in addition to the test matrix and engine control parameters that were varied in the data. Higher $\mathrm{CN}$ and lower AC fuels required a higher percentage CNG for proper combustion phasing than their counterpart fuels. This conclusion suggests that the use of bio-derived diesel fuel as the high reactivity fuel for $\mathrm{RCCl}$ operation would allow for a greater percentage of low reactivity fuel to be used based on the typically higher $\mathrm{CN}$ and low to no AC of bio-derived diesel fuel [50]. Furthermore, aromatic compounds are typically lower in $\mathrm{CN}$, especially those with lower hydrogen intensity, than paraffin and naphthene compounds, which are generally higher in CN [51]. However, this conclusion was not necessarily supported by Hanson, et al. who observed that the use of B20 allowed for reduced low reactivity fuel fraction [26], although it is important to note that the $C N$ of the $B 20$ (46 CN) was the same as the ULSD used in the study.

\subsection{Combustion Characteristics}

Comparing the characteristics of $\mathrm{CDC}$ and $\mathrm{RCCl}$ combustion reveals drastically different combustion processes. Figure 26 plots the in-cylinder pressure and HRR from an RCCI preliminary test with pump diesel and chemically pure $\mathrm{CH}_{4}$, a CDC test, and a CDC test with EGR at the low load operating condition. The in-cylinder pressure curve for $\mathrm{RCCl}$ operation was much larger in magnitude and exhibited a premixed combustion shape compared to the two diesel configurations. Additionally, the HRR curve for $\mathrm{RCCl}$ was broader and was advanced on a crank angle basis from the diesel HRR curves. Note that Figure 26 is provided as a general comparison, to make a direct comparison between the different combustion strategies they should be operated and compared at a similar combustion phasing, for example at the same CA50.

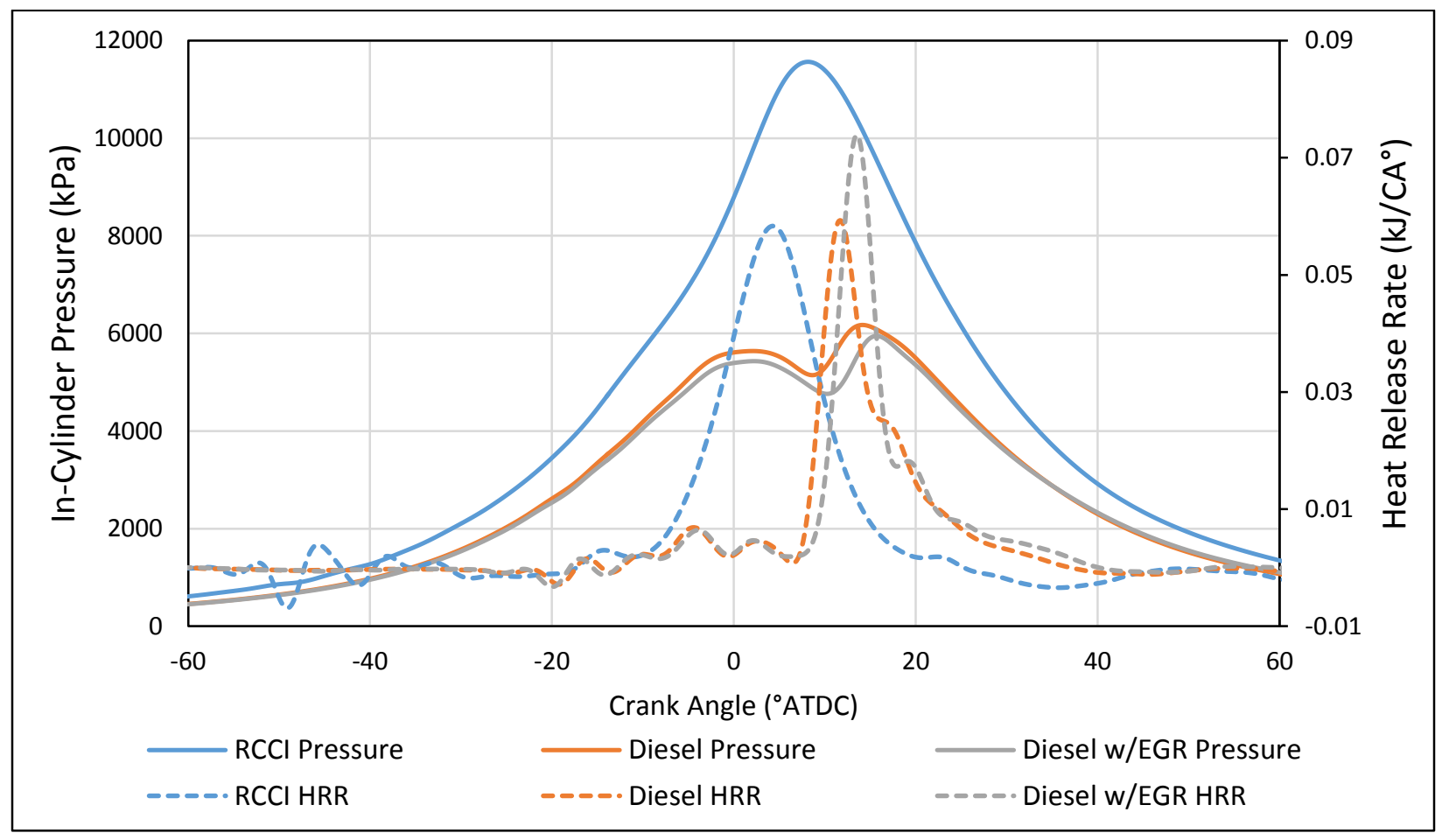

Figure 26: Comparison of In-Cylinder Pressure and HRR for RCCI, CDC, and CDC with EGR

In order to discern fuel property effects on $\mathrm{RCCl}$ combustion characteristics, it is useful to use average and statistical values from combustion processes such as maximum in-cylinder pressure and PRR, 
maximum HRR, combustion duration and COV of IMEP. These values offer a more concise method to characterize $\mathrm{RCCl}$ combustion compared to, for example, examining individual in-cylinder pressure or HRR curves for each test performed for each fuel. However, the shape of the HRR curves can provide insight into the effect of high reactivity fuel properties on RCCI combustion. Thus, average HRR curves from sample tests from each fuel with similar CA50 and COV of CA50 were selected and presented as a means of comparing each fuel's HRR characteristics.

\subsubsection{In-Cylinder Pressure and Pressure Rise Rate}

At the low load operating condition, a trend of decreasing in-cylinder pressure and PRR with increasing $\mathrm{CN}$ was observed. Although the high measurements of in-cylinder pressure and PRR exhibited by the low CN fuels may be related to the retarded DI SOI timing (compared to DI SOI timing of the medium and high CN fuels) that was necessary for these fuels to operate without misfire. Despite the presence of a $\mathrm{CN}$ based trend, there were deviations present within the $\mathrm{CN}$ groups, such as the maximum incylinder pressure for FACE 1 or FACE 5, suggesting that other fuel properties had an influence on the maximum pressure and PRR.

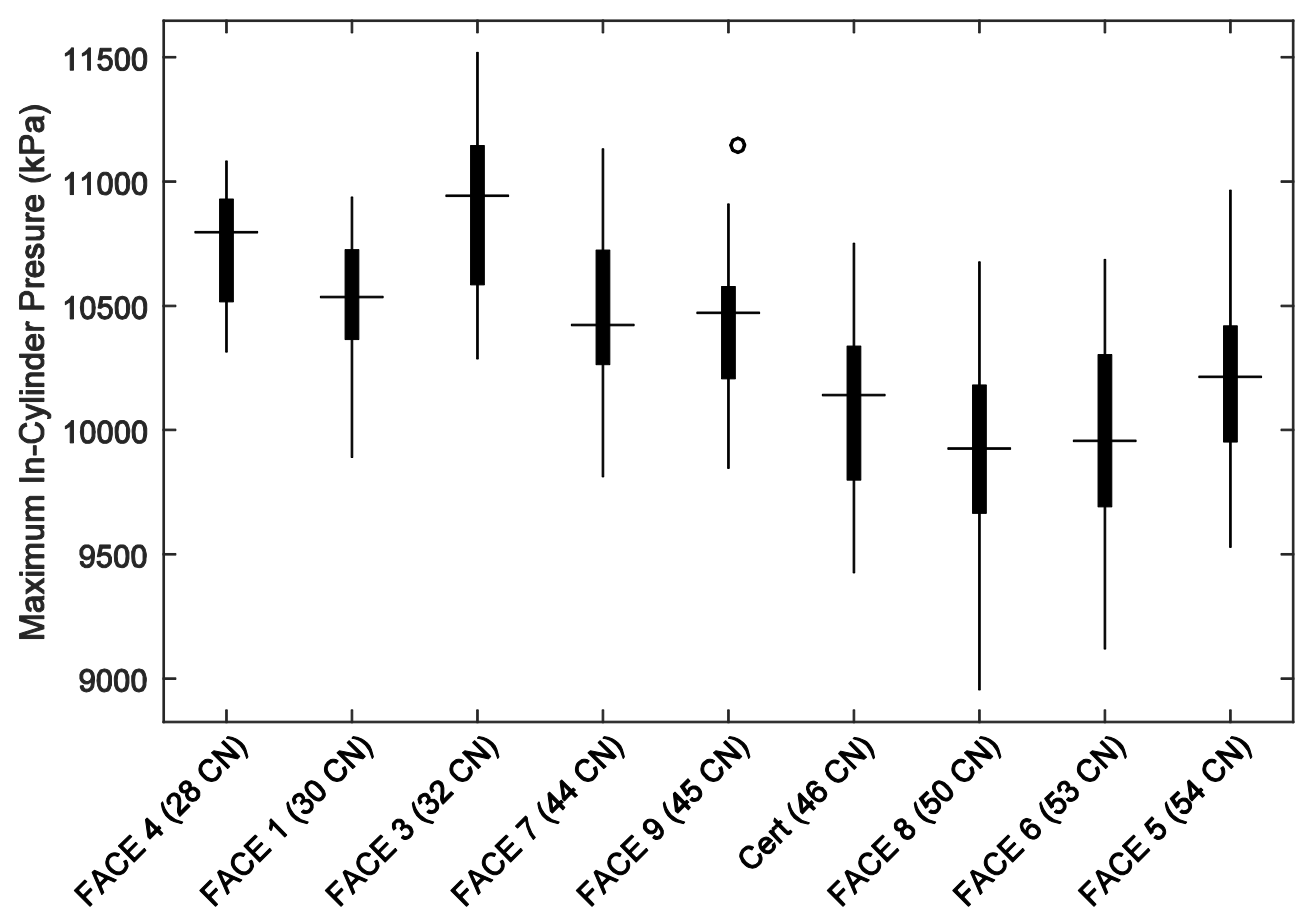

Figure 27: Maximum In-Cylinder Pressure for the Low Load Operating Condition (Varying IMAP and CA50)

As a reminder, the maximum in-cylinder pressure data presented for each of the high reactivity fuels in Figure 27 is from tests with varying IMAP and CA50 set points. A least squares fit applied to the all of the low load in-cylinder pressure data suggested that in addition to CN, AC and T10 were also influential high reactivity fuel properties on $\mathrm{RCCl}$ in-cylinder pressure with a high level of confidence based on $\mathrm{p}$ values less than 0.0001. DI SOI timing, IMAP, and CA50 were also included in the model since they were varied for the test matrix and all retained a $p$ value of less than 0.0001 . Equation 39 describes the model. 


\section{Equation 39: Least Squares Fit Model for Maximum In-cylinder Pressure at the Low Load Operating Condition}

Maximum Pressure

$$
\begin{aligned}
& =10640-361.66 * \text { CA50 }+279.22 * I M A P+9.7578 * A C-30.328 * C N-5.0037 \\
& * T 10+32.900 * \text { DI SOI }
\end{aligned}
$$

The $R^{2}$ value for the least squares fit model was 0.91 . The model suggested that maximum in-cylinder pressure increased with increasing $A C$ and decreasing $T 10$. With respect to $A C$, this trend can be observed in Figure 27. FACE 1 was a low AC fuel and exhibited, on average, lower in-cylinder pressure compared to FACE 4 and FACE 3, which were both high AC fuels. Additionally, in-cylinder pressure for the Cert fuel (low AC) was noticeably lower than the other medium CN fuels, FACE 7 and FACE 9, which both had high AC. The AC based in-cylinder pressure trend can be explained by the notion that aromatics combust at higher temperatures and thus higher pressures compared to paraffin and naphthene hydrocarbons. However, FACE 5 and FACE 6 have low AC, while FACE 8 has a high AC, which contradicts the trend. The lower T10 of FACE 5 and FACE 6 may have contributed to the departure from the $\mathrm{AC}$ trend for high $\mathrm{CN}$ fuels. Figure 28, provides visual evidence that $\mathrm{T} 10$ had a notable influence on in-cylinder pressure. This may be related to the correlation between $\mathrm{CN}$ and T10, as presented in Section 4.7.

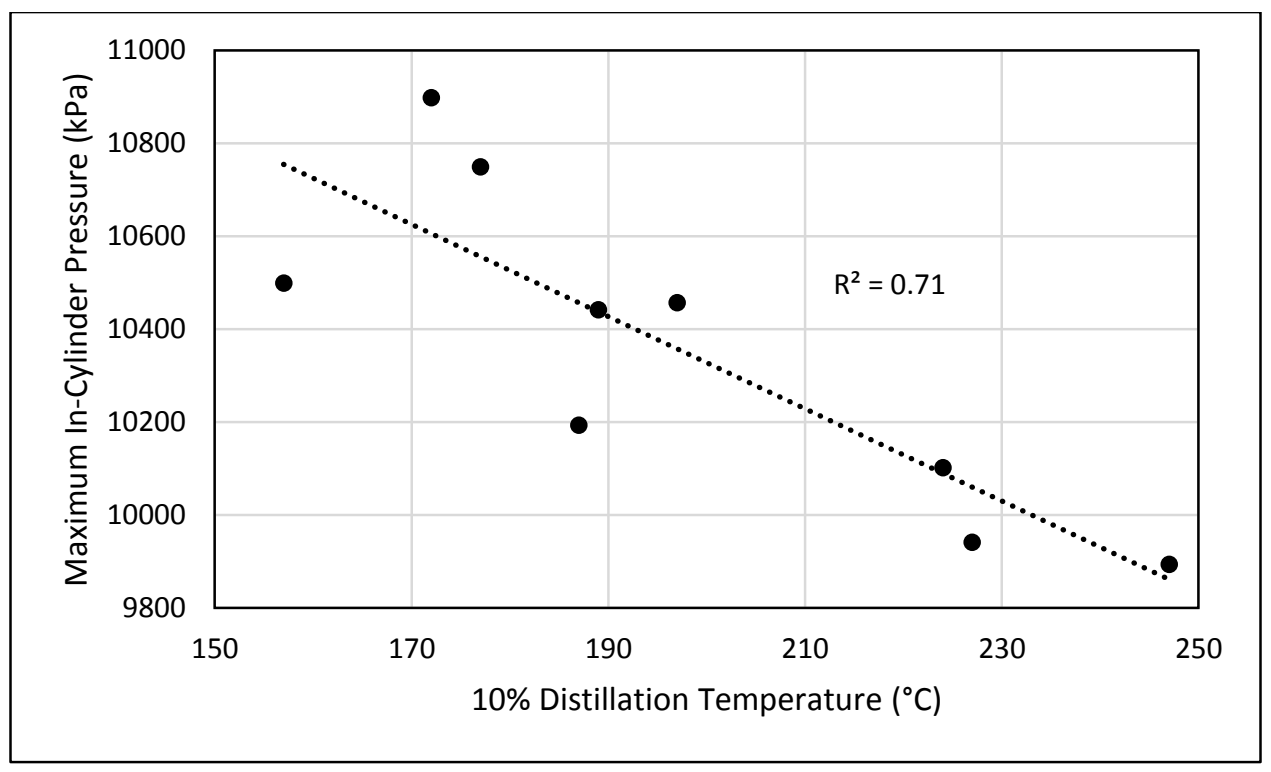

Figure 28: Maximum In-Cylinder Pressure Averages versus T10 for the Low Load Operating Condition 


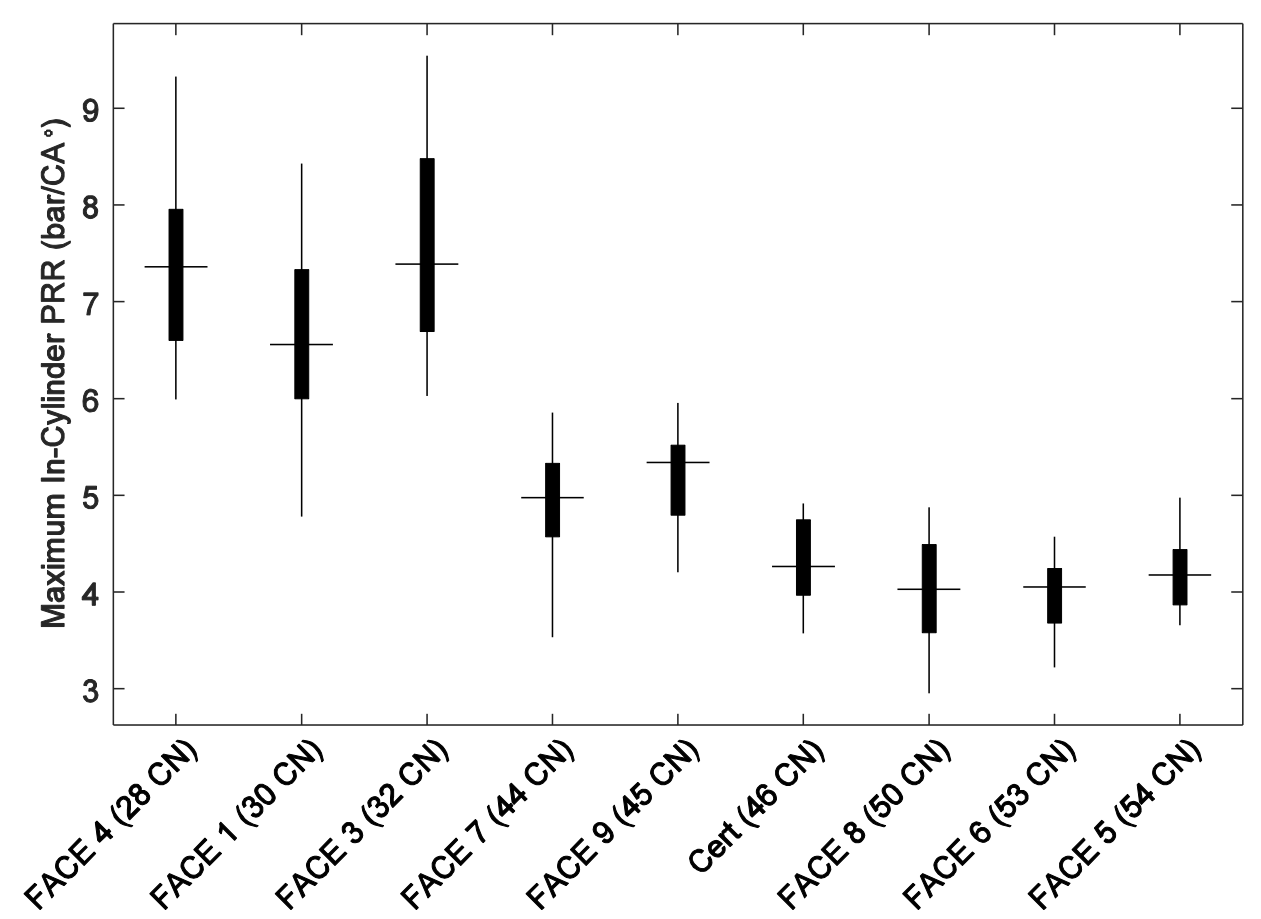

Figure 29: Maximum In-Cylinder PRR for the Low Load Operating Condition (Varying IMAP and CA50)

Trends similar to the maximum in-cylinder pressure can be observed for the PRR at the low load operating condition presented in Figure 29. A decreasing PRR with increasing CN correlation was present as well as an $A C$ and $T 10$ correlation for low and medium $C N$ fuels. A least squares fit model including CN, AC, T10, IMAP, and CA50 resulted in an $R^{2}$ value of 0.77 . DI SOI was not included because of a high $p$ value. The model, described by Equation 40, indicated that lower $A C$ and higher $\mathrm{T} 10$ resulted in lower PRR. However, similar to the maximum pressure, the AC trend was not present for the high $\mathrm{CN}$ fuels.

\section{Equation 40: Least Squares Fit Model for Maximum In-cylinder PRR at the Low Load Operating Condition}

Maximum PRR

$$
\begin{aligned}
& =15.654-0.10714 * C N-0.70463 * C A 50-9.7282 * 10^{-3} * T 10-0.29272 \\
& * I M A P+0.014509 * A C
\end{aligned}
$$




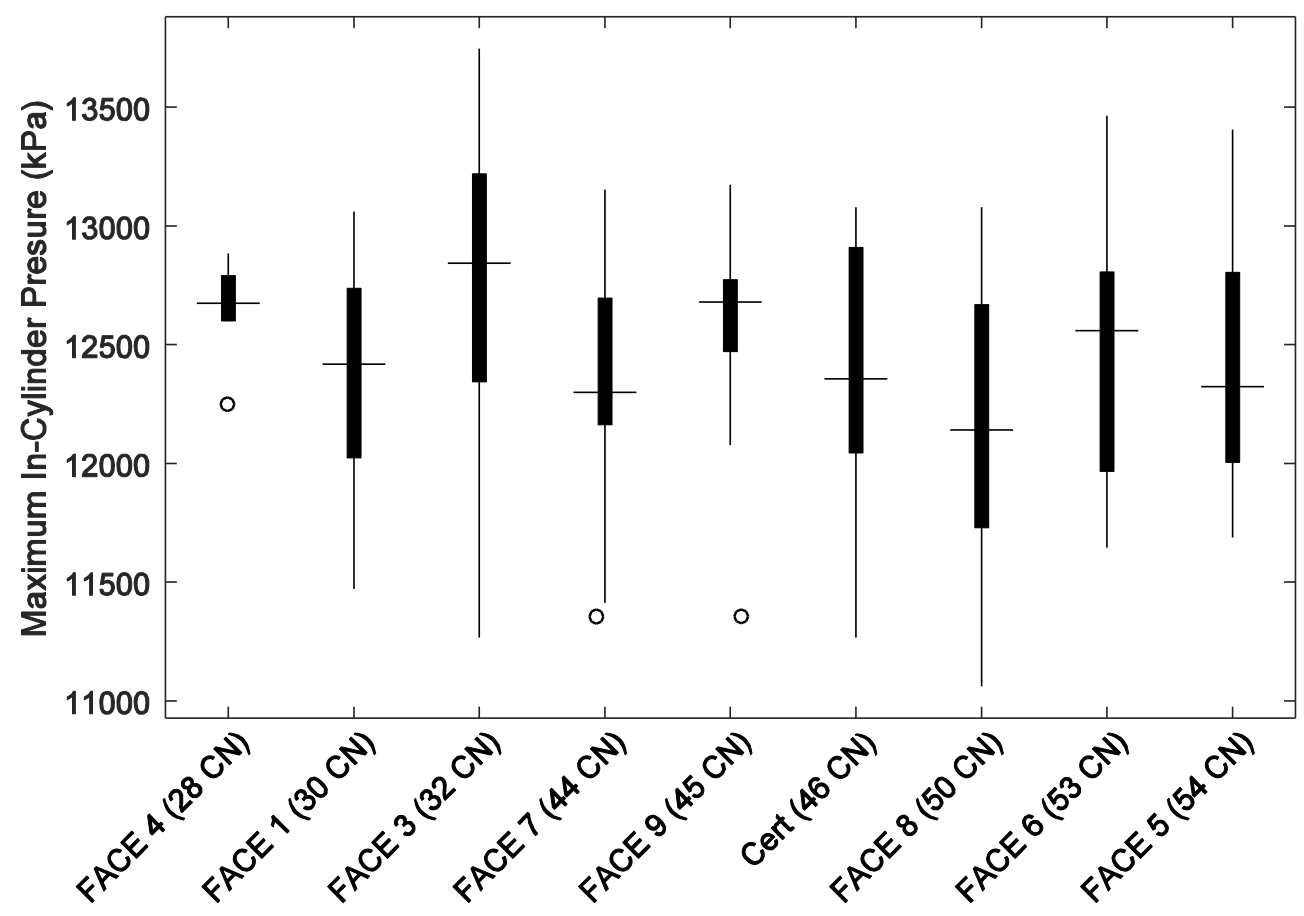

Figure 30: Maximum In-Cylinder Pressure for the High Load Operating Condition (Varying Main DI SOI Timing and CA50)

As a reminder, the maximum in-cylinder pressure data presented for each of the high reactivity fuels in Figure 30 is from tests with varying main DI SOI timing and CA50 set points. The maximum in-cylinder pressure results for the high load operating condition presented in Figure 30 are more convoluted than the low load operating condition results presented in Figure 27. Thus, there were no discernible trends based on the available fuel properties. In fact, several different approaches to regression analyses did not reveal any trends that could be reported with confidence. 


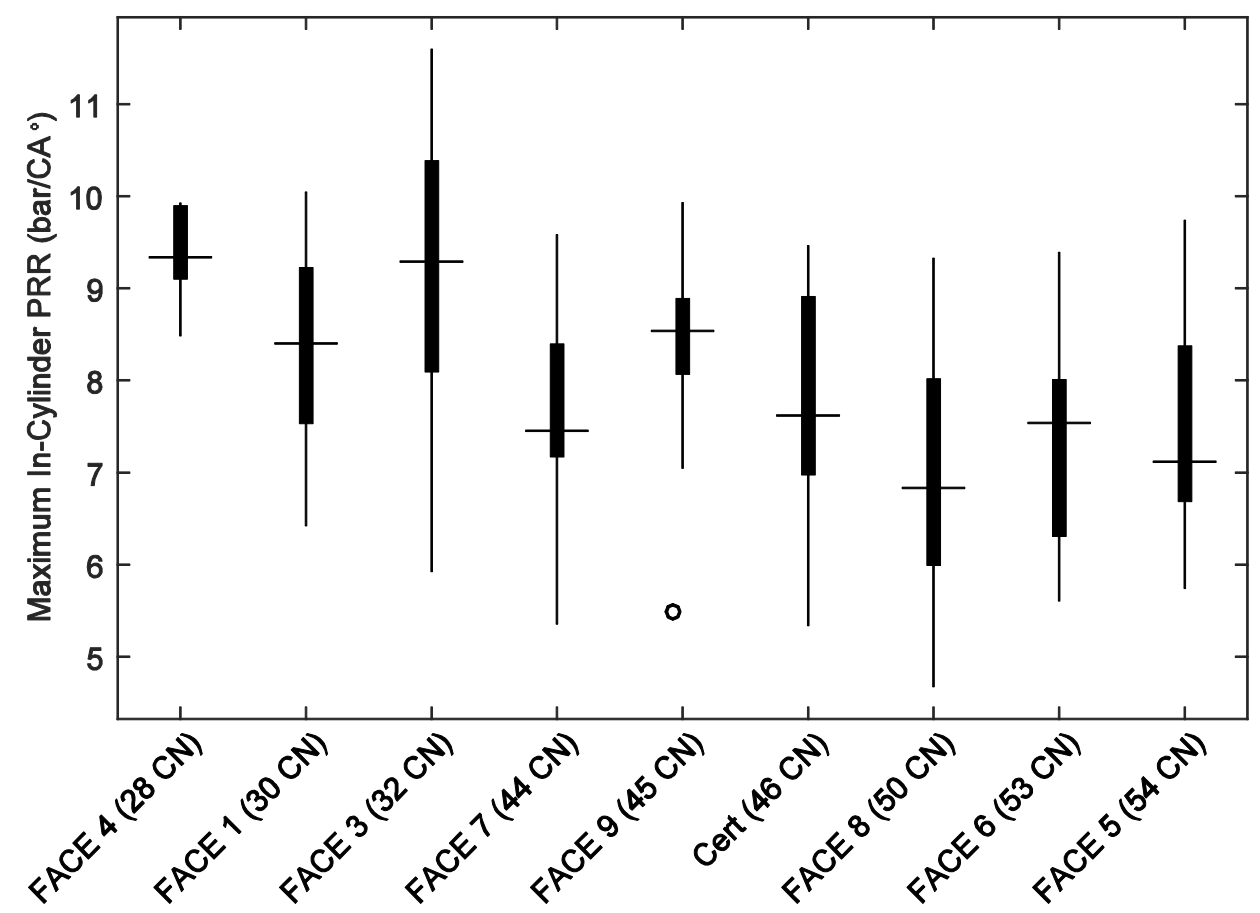

Figure 31: Maximum In-Cylinder PRR for the High Load Operating Condition (Varying Main DI SOI Timing and CA50)

Figure 31 provides visual evidence of a $\mathrm{CN}$ effect on in-cylinder PRR, especially comparing the low $\mathrm{CN}$ fuels to the high CN fuels. A screening analysis performed to determine fuel property influences on maximum in-cylinder PRR at the high load operating condition suggested that $\mathrm{CN}, \mathrm{CA} 50$, and main DI SOI timing were influential with a high level of confidence. However, when applying the least squares fit model main DI SOI timing was not included due to a high $p$ value. The least squares fit model with $\mathrm{CN}$ and CA50 had an $R^{2}$ value of 0.85 and is described by Equation 41.

\section{Equation 41: Least Squares Fit Model for Maximum In-cylinder PRR at the High Load Operating Condition}

$$
\text { Maximum } P R R=16.106-1.0355 * C A 50-0.082476 * C N
$$

The observation of decreasing in-cylinder PRR as $\mathrm{CN}$ of the high reactivity fuel increases, which is also shown in Figure 29 at the low load operating condition, agrees with the conclusion made by Nieman, et al. that a higher reactivity difference between the low and high reactivity fuels results in lower incylinder PRR [25]. Although Nieman, et al. achieved this higher reactivity difference by using a higher octane low reactivity fuel, natural gas compared to gasoline, the effect is the same as using a higher $\mathrm{CN}$ high reactivity fuel as displayed herein.

\subsubsection{Heat Release Rate and its Derivatives}

The HRR of the combustion process was derived from the measured in-cylinder pressure and corresponding combustion chamber volume. The shape of the HRR curve with respect to crank angle provides valuable information on the combustion process and can be used to understand exhaust emissions and engine performance phenomena. Additionally, derivatives from the HRR calculations, 
such as MFB, provide further means to define combustion characteristics and parameters that can be used to benchmark combustion strategies, fuels, and control parameter adjustments. All the HRR data presented in this section is on a gross basis, e.g. including the heat loss to the cylinder walls.

Results from a screening analysis and subsequent least squares fit of maximum heat release rate values for all the high reactivity fuels indicated that $\mathrm{CN}$ was influential at high and low load operating conditions with a high degree of certainty. To visualize this phenomena one test from each fuel at each load condition was selected that exhibited an average CA50 between 4.00 and 4.25 CA $^{\circ}$ ATDC in conjunction with the lowest COV of CA50 from cycle-to-cycle. Figure 32 displays the HRR curves from these tests for the low load operating condition.

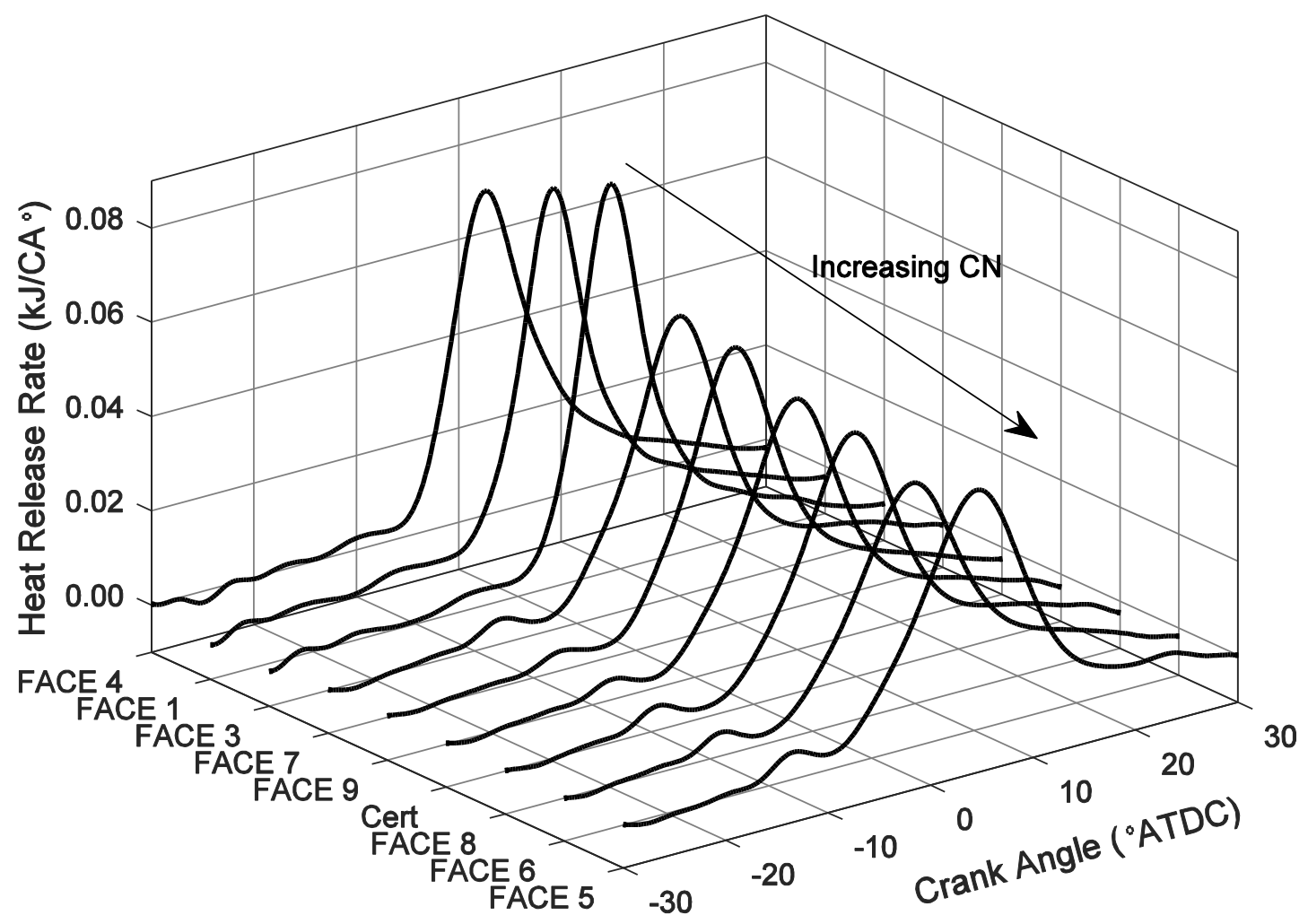

Figure 32: Sample HRR from Each High Reactivity Fuel during Low Load Operation

At the low load operating condition, the HRR traces plotted in Figure 32 exhibit discernible changes in peaks and overall shape as the $\mathrm{CN}$ of the high reactivity fuel changes. Foremost, a trend of decreasing maximum $\mathrm{HRR}$ rate as a function of increasing $\mathrm{CN}$ of the high reactivity fuel was clearly evident, especially when comparing the low CN fuels to the medium and high CN fuels. Additionally, low LTHR reactions were distinctly present for the high and medium $C N$ fuels, whereas LTHR reactions were much more subdued for $\mathrm{RCCl}$ operation with the low $\mathrm{CN}$ fuels. The LTHR reactions are represented by the small hump in the HRR occurring between approximately -10 CA $^{\circ}$ ATDC and TDC. Furthermore, the LTHR reactions began at an earlier $\mathrm{CA}^{\circ}$ for the high $\mathrm{CN}$ fuels compared to the medium $\mathrm{CN}$ fuels, which was a function of the reduced ignition delay provided by a high $\mathrm{CN}$ high reactivity fuel. This observation is supported by an earlier average CA5 for the high CN fuels (-4.95 CA ${ }^{\circ}$ ATDC) versus the medium CN fuels 
(-3.91 $\left.\mathrm{CA}^{\circ} \mathrm{ATDC}\right)$ and the low $\mathrm{CN}$ fuels (-1.91 $\left.\mathrm{CA}^{\circ} \mathrm{ATDC}\right)$. This, in addition to the overall lower maximum HRRs, resulted in a longer combustion duration as higher $\mathrm{CN}$ fuels were used which is displayed in Figure 33.

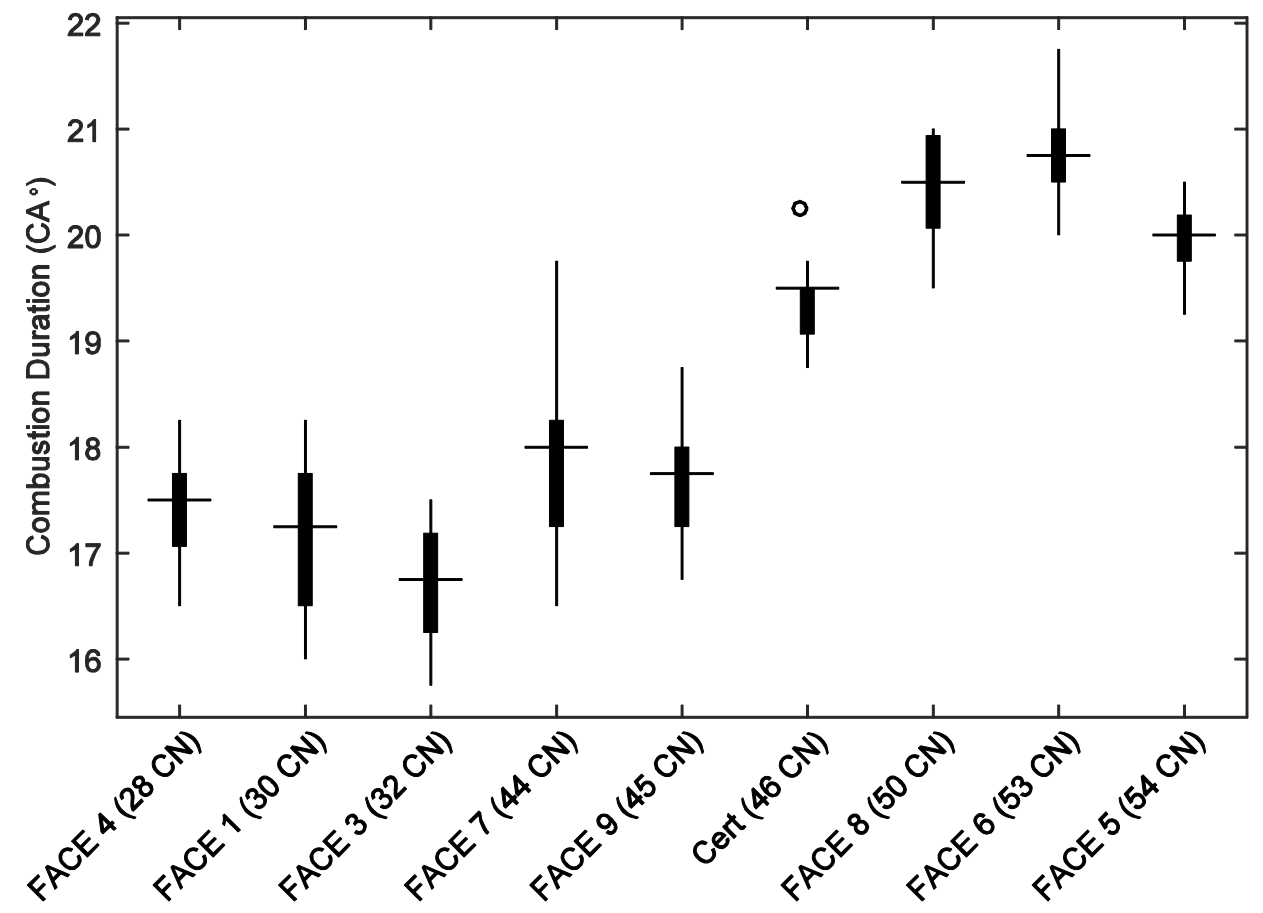

Figure 33: Combustion Duration (CA5 to CA95) at the Low load Operating Condition Ordered by CN (Varying IMAP and CA50)

Combustion duration, as plotted in Figure 33, was defined as the $\mathrm{CA}^{\circ}$ that passed from CA5 until CA95. As noted, a general correlation with $\mathrm{CN}$ was observed, where combustion duration increased with the use of higher $\mathrm{CN}$ high reactivity fuels. A screening analysis and subsequent least squares fit model of the data suggested that in addition to $\mathrm{CN}, \mathrm{AC}$ and T10 were influential high reactivity fuel properties on the combustion duration. IMAP and DI SOI timing were also included in the model, which exhibited a $\mathrm{R}^{2}$ value of 0.93 . The model is defined by Equation 42 .

Equation 42: Least Squares Fit Model for Combustion Duration at the Low Load Operating Condition

\section{Combustion Duration}

$$
\begin{aligned}
& =12.616+0.19542 * C N+0.026374 * T 10-0.23818 * D I S O I+0.59901 * I M A P \\
& -0.020097 * A C-0.22193 * C A 50
\end{aligned}
$$

The least squares fit model suggested that as AC decreases, combustion duration increased, although this trend was not easily observable potentially due to the dominant influence of CN and T10.

Examining the fuels with low AC within the low CN fuels (FACE 1), medium CN fuels (Cert), and high CN fuels (FACE 6 and FACE 5) does not provide a clear trend. Furthermore, the contribution from AC to the calculated combustion duration from the least squares fit model was minimal compared to the contribution by $\mathrm{CN}$ and T10. The contribution of these properties can be calculated by multiplying the range of $A C$ of the fuels tested by the estimated coefficient for $A C$ generated by the least squares fit. 
The contribution for $\mathrm{AC}$ equated to -0.209 to $-1.00 \mathrm{CA}^{\circ}$ compared to 4.14 to $6.51 \mathrm{CA}^{\circ}$ for $\mathrm{T} 10$ and 5.55 to 10.6 $\mathrm{CA}^{\circ}$ for $\mathrm{CN}$ using the same approach. Hence, the influence of $\mathrm{CN}$ and T10 were much more substantial given their contribution to the least squares fit model. Additionally, the effect of T10 can be observed in Figure 34.

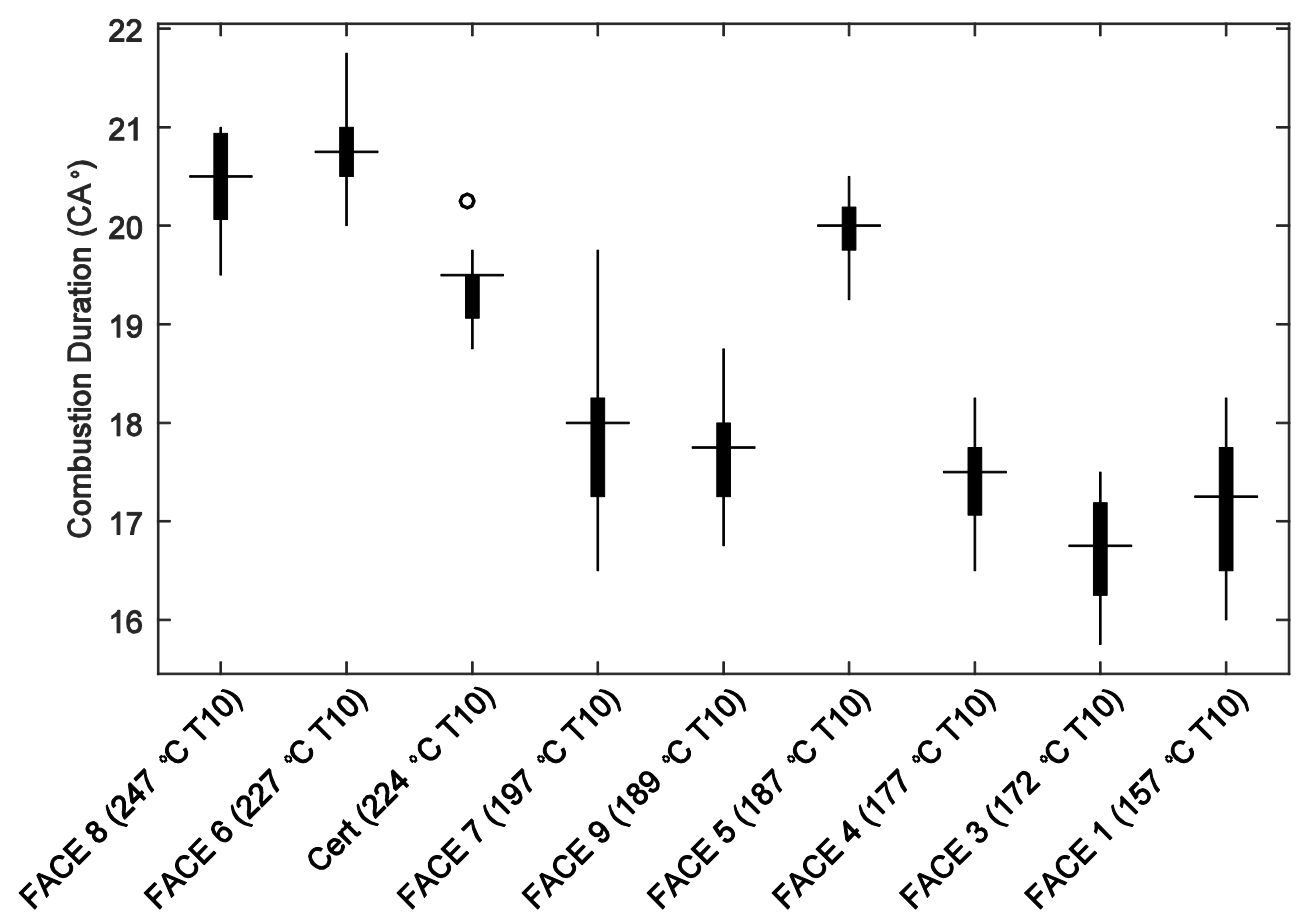

Figure 34: Combustion Duration (CA5 to CA95) at the Low load Operating Condition Ordered by T10 (Varying IMAP and CA50)

With the exception of FACE 5, a trend of decreasing combustion duration as T10 decreases can be observed in Figure 34. The exception of FACE 5 from the overall trend may be related to its high $\mathrm{CN}$, but note that compared to the other high CN fuels (FACE 8 and FACE 6) it exhibited less combustion duration on average. Given that it had the highest $\mathrm{CN}$, yet a lower average combustion duration suggests that T10 is influential and not a result of the correlation between $\mathrm{CN}$ and T10.

As noted above, the combustion duration observed during the low load operating condition was directly related to the HRR exhibited by the combustion of the various high reactivity fuels and natural gas.

Comparing, Figure 35 and Figure 36 below to Figure 33 and Figure 34, respectively, demonstrates that the maximum HRR was inversely proportional the combustion duration. 


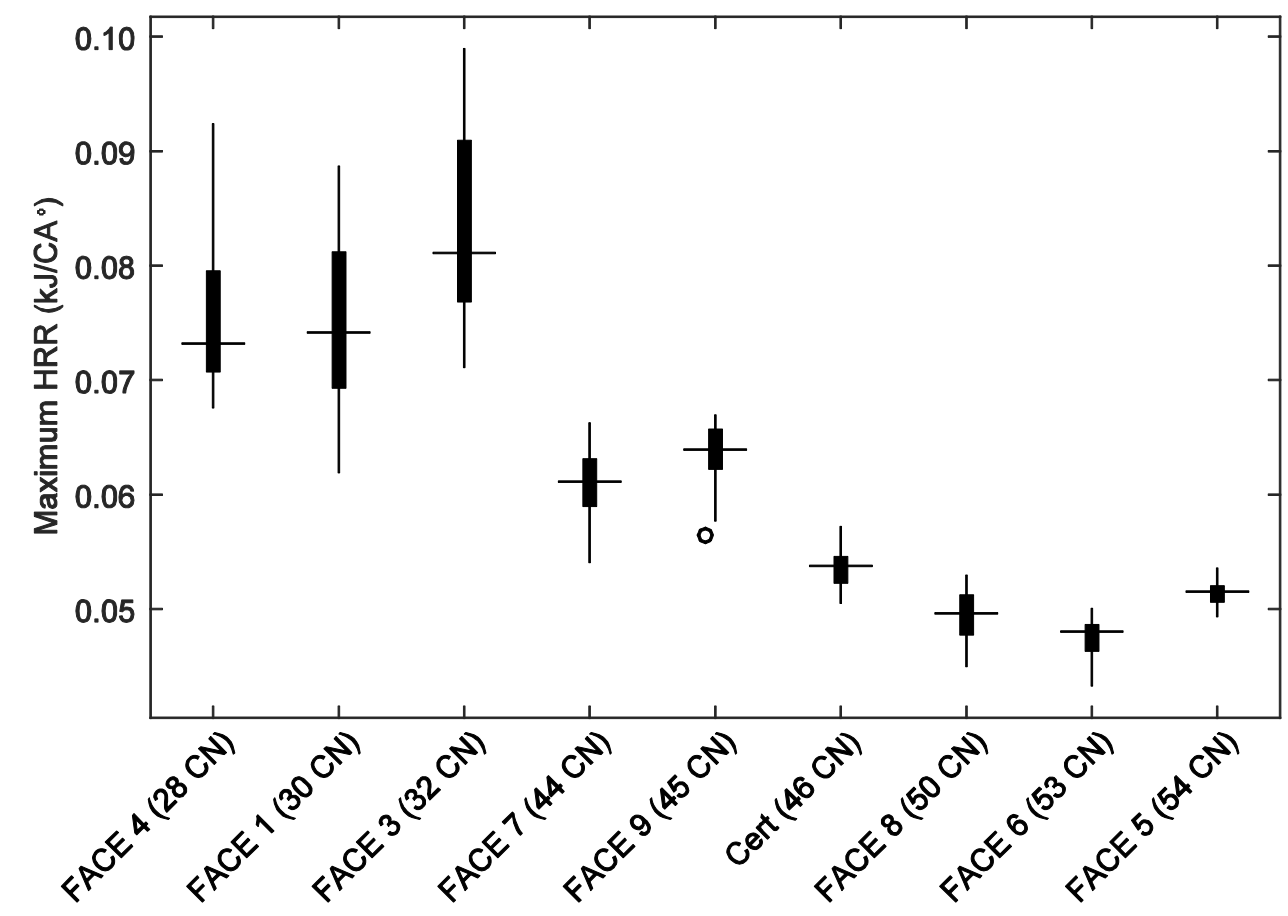

Figure 35: Maximum HRR for at the Low load Operating Condition Ordered by CN (Varying IMAP and CA50)

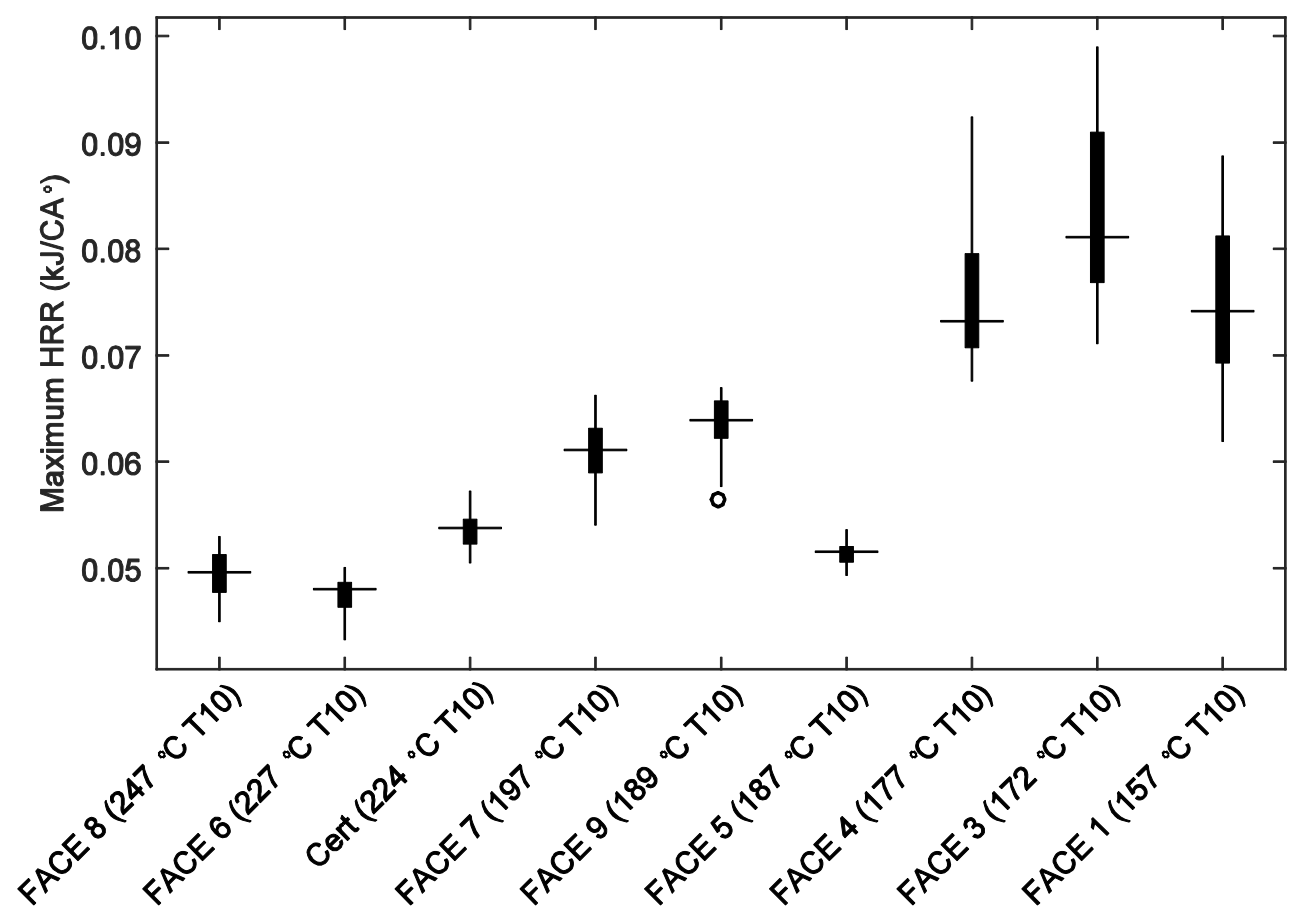

Figure 36: Maximum HRR for at the Low load Operating Condition Ordered by T10 (Varying IMAP and CA50) 
The same approach for analyzing the HRR and its derivatives at the low load operating condition was followed for the high load operating condition. Figure 37 represents HRR traces from one test from each fuel that was selected based on an average CA50 between 4.00 and $4.25 \mathrm{CA}^{\circ}$ ATDC in conjunction with the lowest COV of CA50 from cycle-to-cycle. Figure 38 displays the maximum HRR for tests at the high load operating condition.

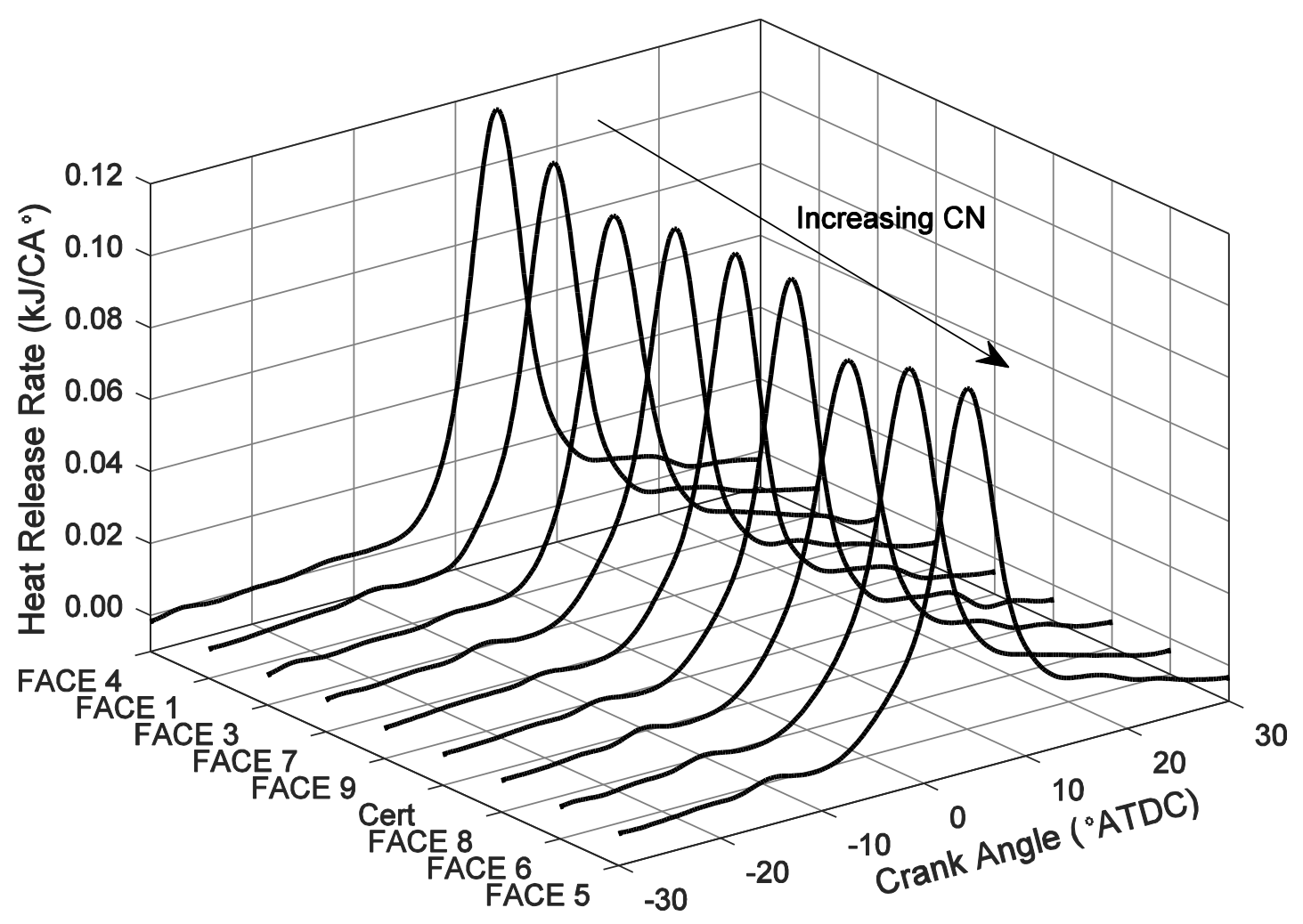

Figure 37: Sample HRR from Each High Reactivity Fuel during High Load Operation 


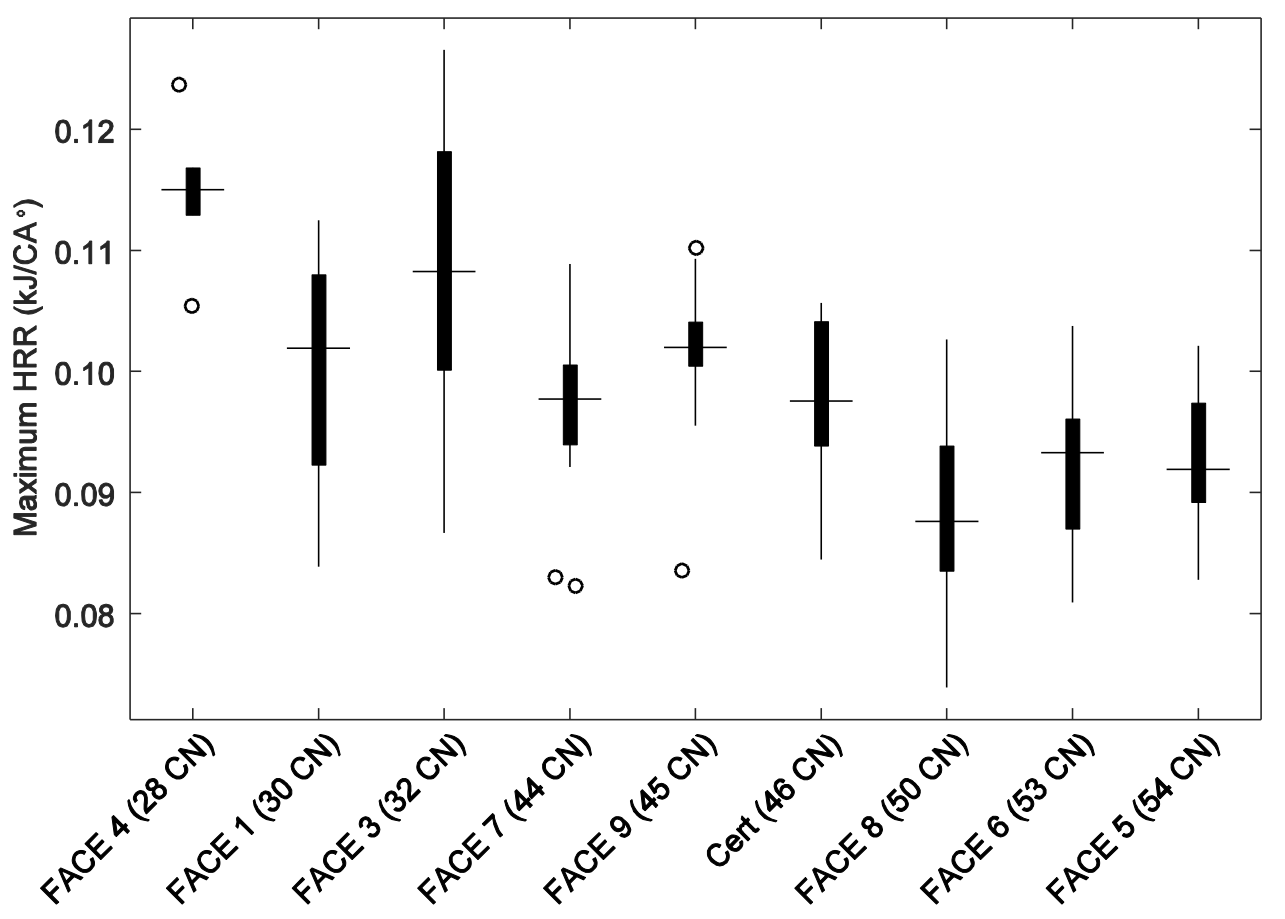

Figure 38: Maximum HRR for at the High load Operating Condition Ordered by CN (Varying Main DI SOI Timing and CA50)

Similar to the low load operating condition, it was apparent from Figure 37 and Figure 38 that CN was influential on the HRR at the high load operating condition. As CN increased, the LTHR became more apparent as well as more advanced with respect to $C A^{\circ}$. Although a trend of maximum HRR decreasing as $\mathrm{CN}$ increased was apparent moving from the low to medium to high $\mathrm{CN}$ groups, within the groups themselves maximum HRR did not necessarily follow $\mathrm{CN}$. Investigating other fuel property effects with JMP resulted in a least squares fit model, defined by Equation 43, that included T10 in addition to CN, both with a high level of confidence ( $p$-value less than 0.0001). CA50 was also included in the model, which had a $R^{2}$ value of 0.72 . Other fuel properties such as AC, T90, and SG did not significantly improve the $R^{2}$ value of the model and an estimate of their coefficients could not be made with a high confidence (i.e. high $p$ values).

\section{Equation 43: Least Squares Fit Model for Maximum HRR at the High Load Operating Condition}

$$
\begin{aligned}
& \text { Maximum } H R R \\
& =0.15918-7.7710 * 10^{-4} * C N-5.4347 * 10^{-3} * C A 50-3.9731 * 10^{-6} \\
& \text { * }(T 10-198.55)^{2}
\end{aligned}
$$

Note that the term for T10 is second order, which can be explained visually by Figure 39. Tracing through the medians provides a function resembling a concave parabola, with the exception of FACE 5. Similar to the maximum HRR data from the low load operating condition, the CN from FACE 5 was more influential than its T10 dictating its low maximum HRR. Additionally, the more retarded DI SOI timing for the low CN fuels and the limited tests performed for FACE 4 due to excessive in-cylinder PRRs may also have contributed to the parabolic shape. 


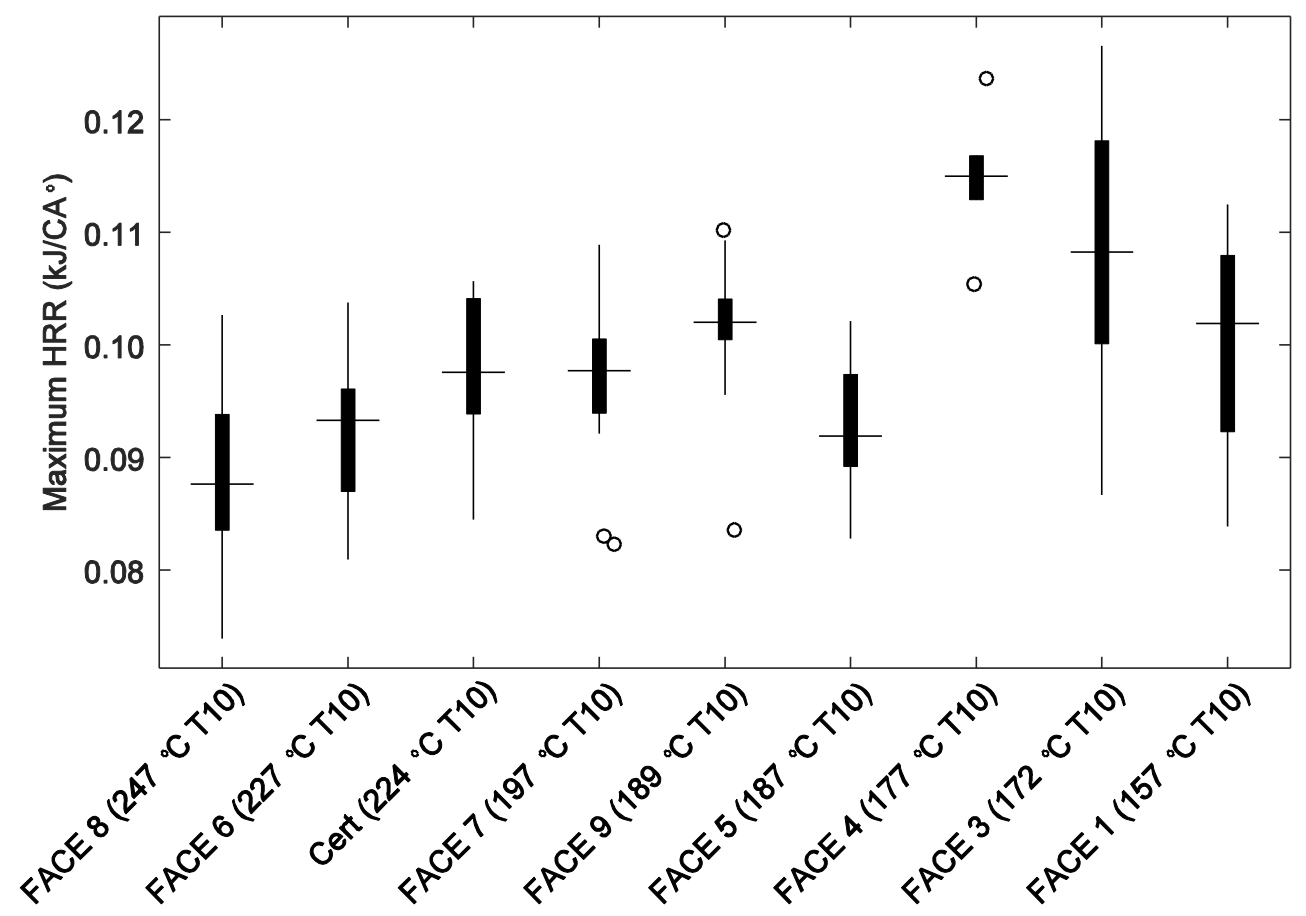

Figure 39: Maximum HRR for at the High load Operating Condition Ordered by T10 (Varying Main DI SOI Timing and CA50)

Unlike the low load operating condition, maximum HRRs from the high load operating condition do not necessarily correspond to the combustion duration. Figure 40 and Figure 41 demonstrate that there was no clear trend of combustion duration with regards to $\mathrm{CN}$ or T10. A least squares fit based on the parameters identified in a screening analysis produced a much more complex equation for combustion duration compared to maximum HRR. The model for combustion duration, detailed in Equation 44, exhibited an $R^{2}$ value of 0.70 and all of the parameter coefficients were estimated with a high level of confidence ( $p$-value less than 0.0001 ). It suggested that SG was very influential on the combustion duration at the high load operating condition, although this could not be visually confirmed. Without a visualization of the specific fuel property influences, conclusions could not be stated with enough confidence to attribute combustion duration trends at the high load operating condition to fuel property effects.

Equation 44: Least Squares Fit Model for Combustion Duration at the High load Operating Condition Combustion Duration

$$
\begin{aligned}
& =85.186-91.315 * S G+0.051762 * T 10-0.011006 *(C N-43.639)^{2}+1113.0 \\
& *(S G-.83705)^{2}+0.036407 * A C-0.081128 * \operatorname{main} \text { DI SOI }
\end{aligned}
$$




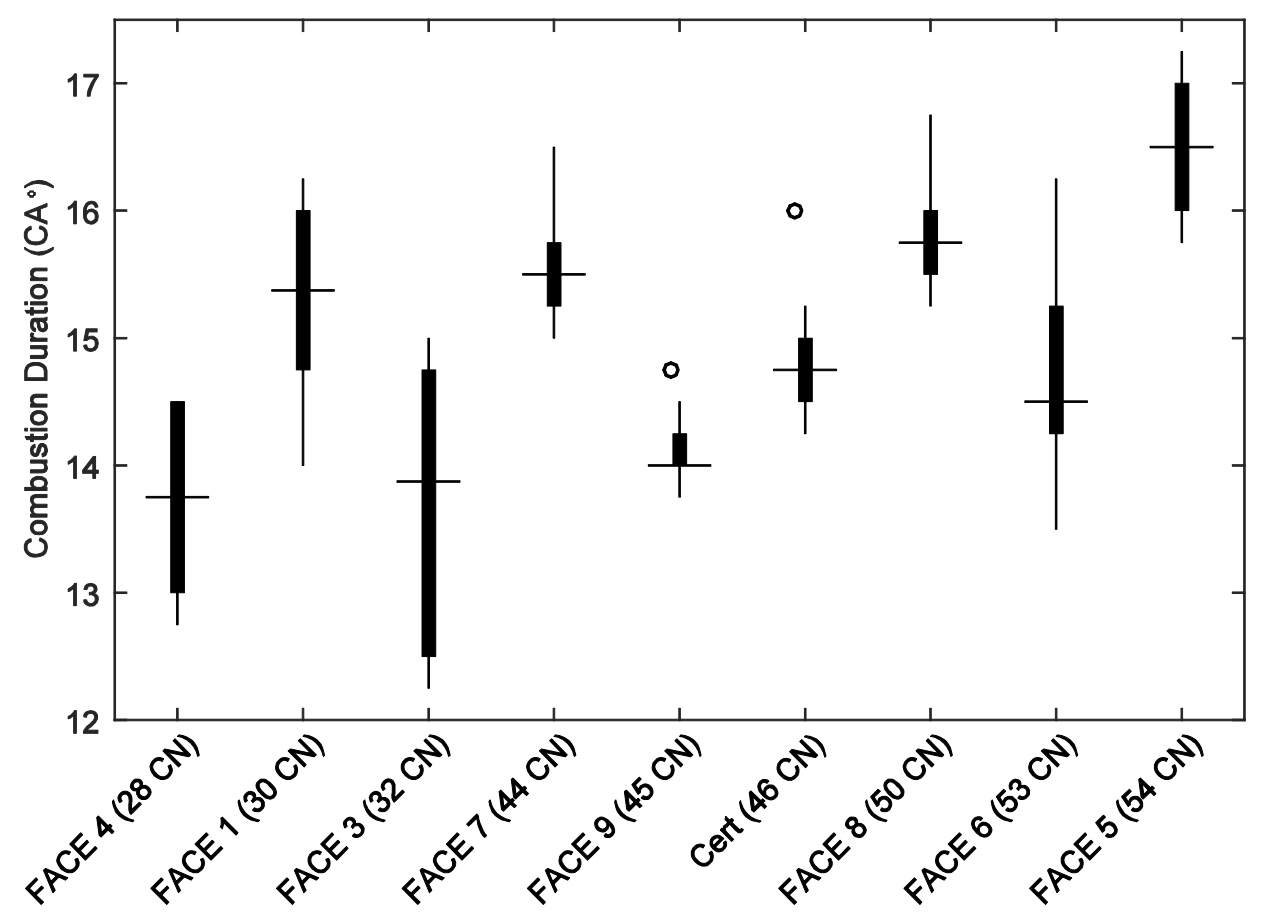

Figure 40: Combustion Duration (CA5 to CA95) at the High load Operating Condition Ordered by CN (Varying Main DI SOI Timing and CA50)

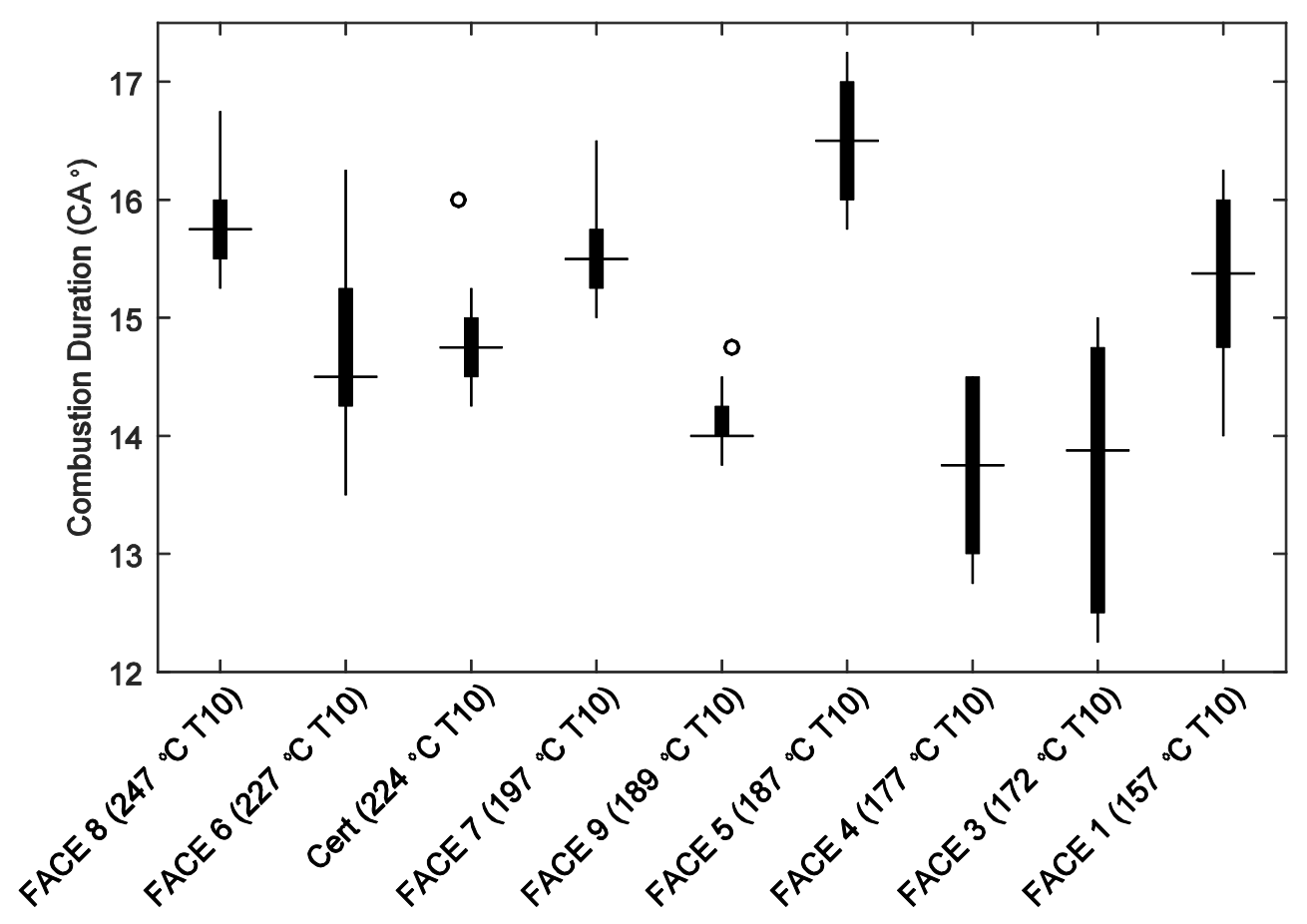

Figure 41: Combustion Duration (CA5 to CA95) at the High load Operating Condition Ordered by T10 (Varying Main DI SOI Timing and CA50)

71 


\subsubsection{Combustion Stability}

Combustion stability can be defined in terms of cycle-to-cycle variability and cylinder-to-cylinder variability for multi-cylinder engines. Cylinder-to-cylinder variability was investigated previously as documented in Section 5.1. Alterations were made to the DI and PFI durations on a cylinder-by-cylinder basis in an effort to balance the maximum in-cylinder pressure observed for all four cylinders. Due to the availability of only one in-cylinder pressure transducer and the limited amount of high reactivity test fuel, cylinder-to-cylinder variations could not be further examined during the high reactivity fuel comparison experiments. However, cycle-to-cycle variability could be examined among operation on the different high reactivity fuels. There are many different metrics to quantify cycle-to-cycle variability and thus combustion stability. The COV of the IMEP per cycle is widely used and it has been documented that when this metric exceeds 10 percent, vehicle drivability problems can arise [11]. Many ICE researchers also use THC and CO emissions as a measure of combustion instability, but these parameters will be discussed later. The COV of IMEP at the low load operating condition is presented in Figure 42.

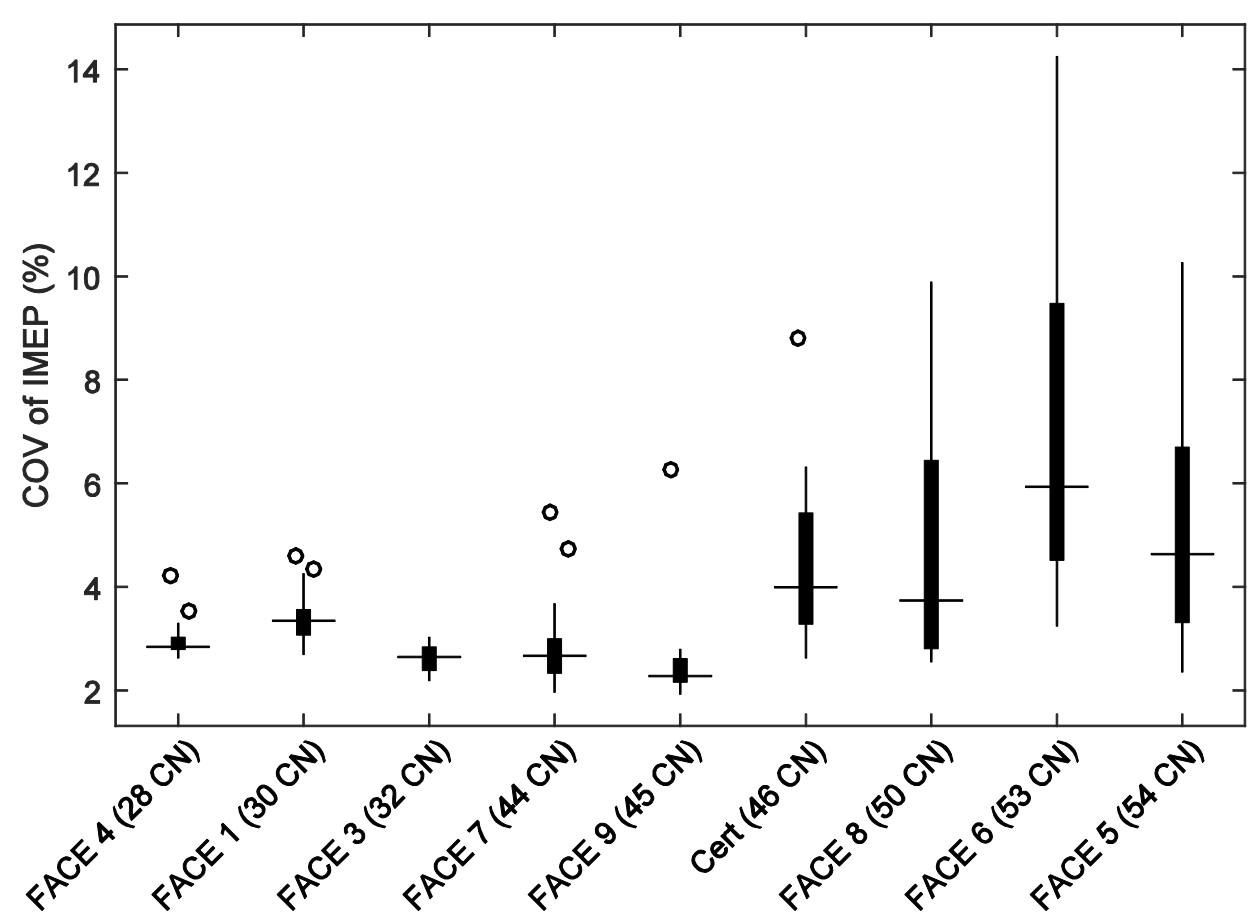

Figure 42: COV of IMEP for the Low Load Operating Condition (Varying IMAP and CA50)

The high CN fuels exhibited the highest COV of IMEP as demonstrated by Figure 42. An explanation for this phenomenon is provided by Figure 43, which makes a compelling argument that the COV of IMEP was an exponential function of the percentage of CNG. As detailed in Section 6.1, the high CN fuels required a higher percentage of $\mathrm{CNG}$ to achieve the target $\mathrm{CA} 50$ values, compared to lower $\mathrm{CN}$ fuels. It is important to note that the low CN fuels utilized retarded DI SOI timing to eliminate misfire, which if not performed would have surely resulted in very large COV of IMEP values. Additionally, it is possible that the high CN fuels may have benefitted from further advancement of the DI SOI timing to retard combustion phasing rather than increased percentage CNG. It was observed during testing at the high load operating condition that advancing the DI SOI timing caused the combustion event to retard and 
thus less percentage CNG was needed to meet the CA50 targets. Equation 45 demonstrates the exponential relationship between COV of IMEP and percentage CNG, which had an $R^{2}$ value of 0.74 .

\section{Equation 45: COV of IMEP as a Function of Percentage CNG Trend line in Figure 43}

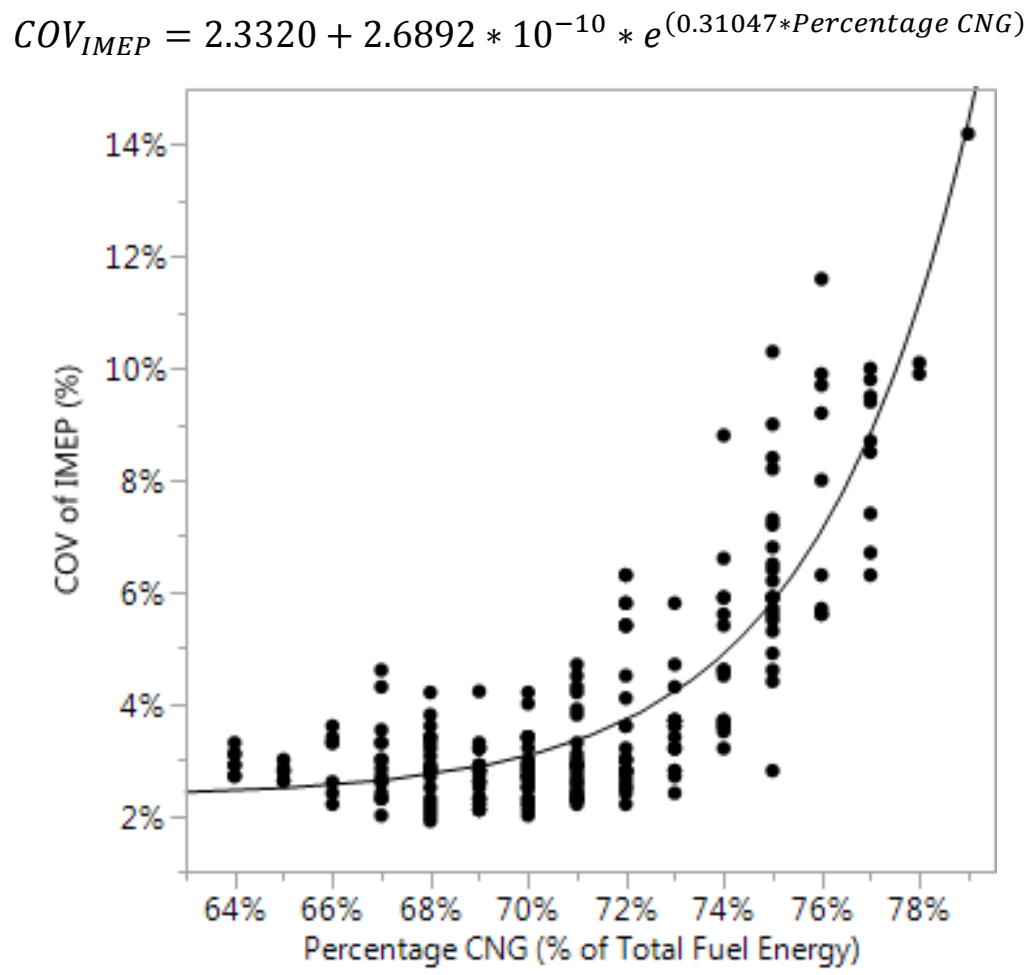

Figure 43: COV of IMEP versus Percentage CNG for the Low Load Operating Condition

The test with a COV of IMEP of approximately 14 percent for FACE 6 was investigated to determine the cause of the high COV of IMEP. It was determined that to achieve the most retarded CA50 target of the test matrix, $5 \mathrm{CA}^{\circ}$ ATDC, a high rate of percentage CNG was used that resulted in an occasional misfire. Figure 44 provides an example of this in the form of two consecutive unfiltered in-cylinder pressure curves from the test with the highest COV of IMEP. The two curves represent a cycle for which misfire occurred, and the next cycle, which did not result in misfire. As noted previously, it is speculated that an advancement in DI SOI timing would have allowed the target CA50 to be achieved without such high percentage CNG that led to misfire. However, this theory was not investigated due to time and fuel quantity constraints. Additionally, the misfire was not observed during data collection and therefore was not addressed at that time. 


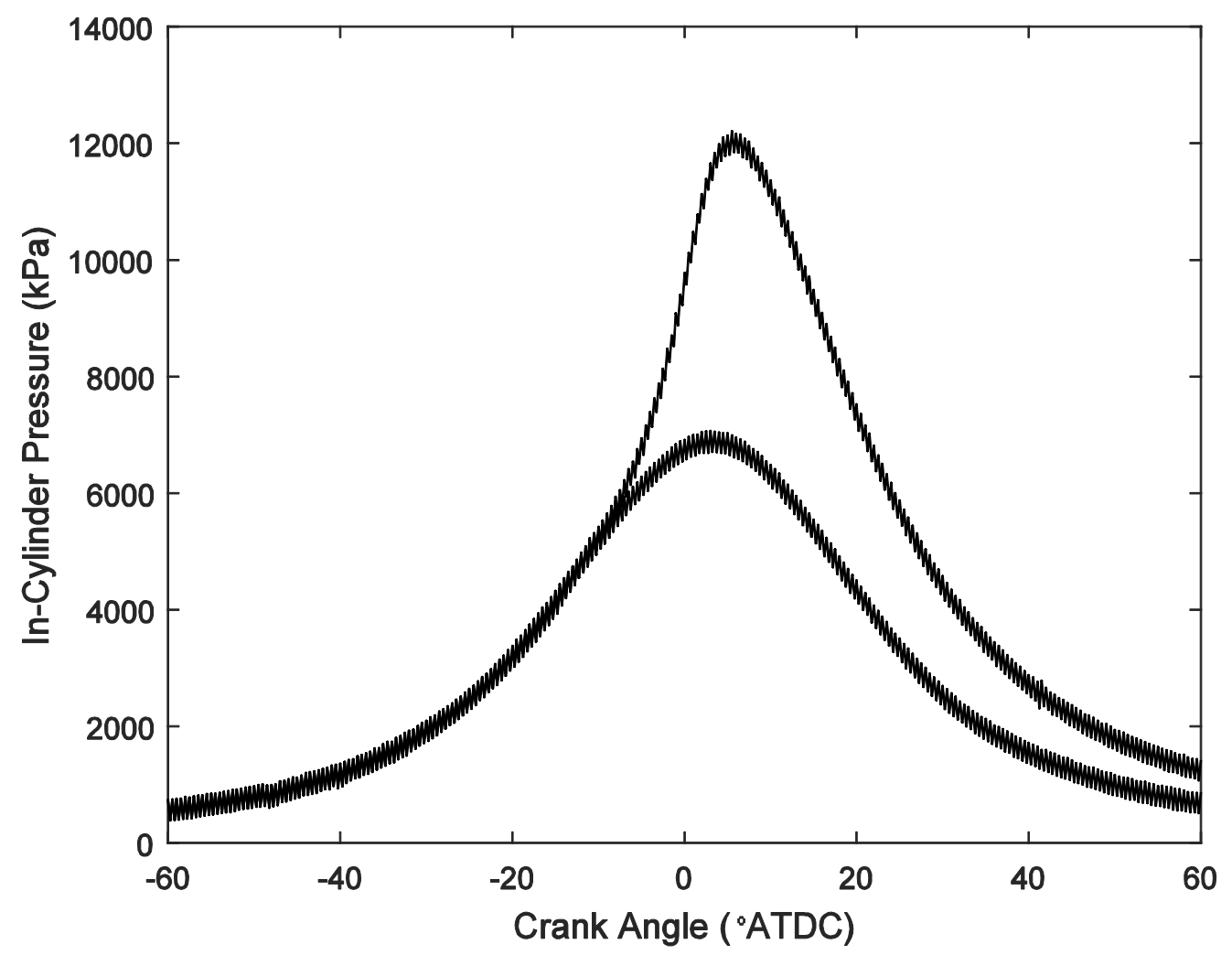

Figure 44: Unfiltered In-Cylinder Pressure Trace for a Cycle with Misfire and a Subsequent Cycle from RCCI Operation with FACE 6 at the Low Load Operating Condition

At the high load operating condition the COV of IMEP trend reverses compared to what was shown for the low load operating condition. Figure 45 reveals that low CN fuels exhibited a higher COV of IMEP with the exception of outliers for the other fuels at the high load operating condition. The DI SOI timing was retarded at this load as well for the low CN fuels, compared to the DI SOI timing for medium and high CN fuels. Similar to the low load operating condition, Figure 46 demonstrates that for high values of percentage CNG the COV of IMEP rises exponentially, although the fit of the trend line equation only resulted in an $R^{2}$ of 0.50 . The tests with high percentage CNG were also the tests that comprised the outlier points (small circles) in Figure 45 for the medium and high $\mathrm{CN}$ fuels. Investigation of fuel property effects, besides $\mathrm{CN}$, on the COV of IMEP at both the high and low load operating conditions did not reveal consistent trends with a high level of confidence. 


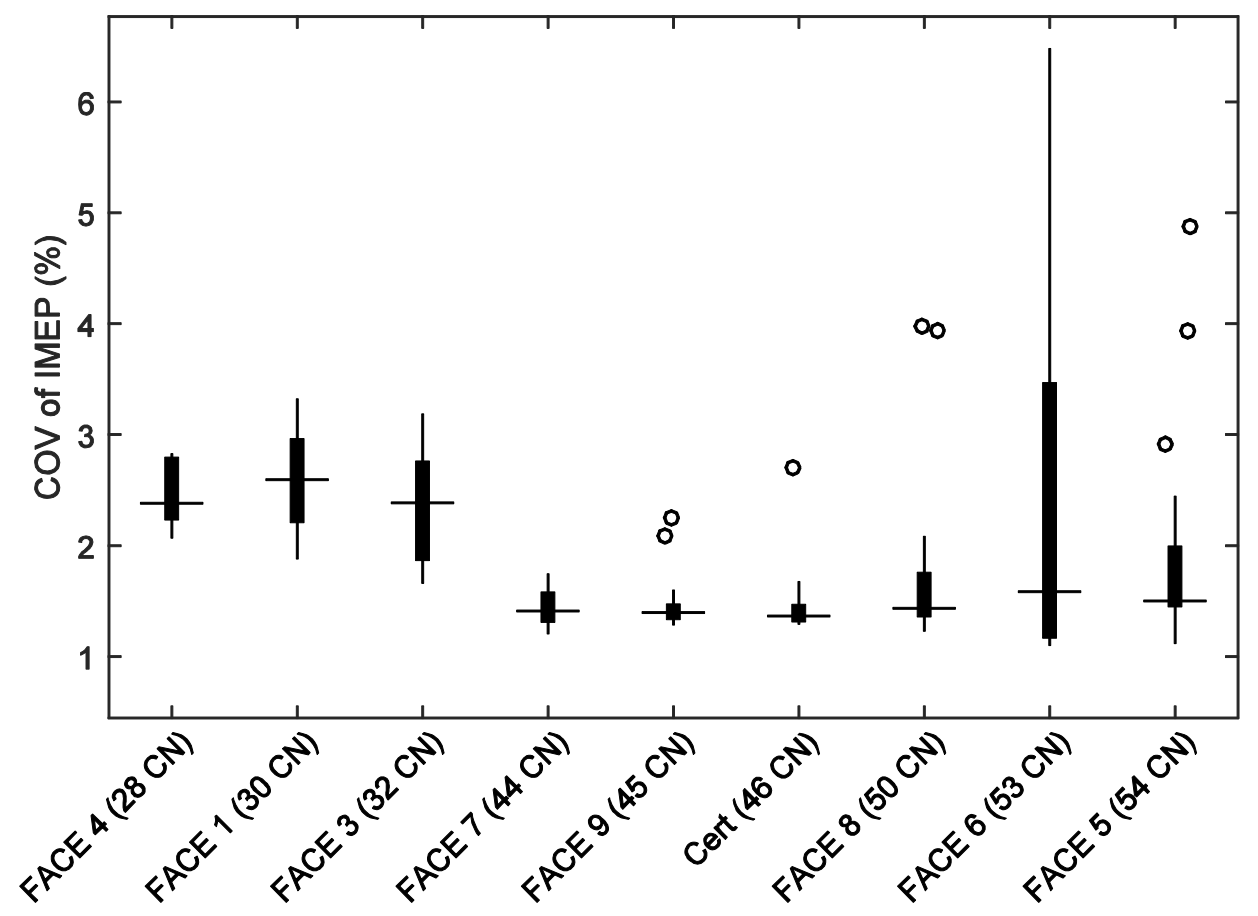

Figure 45: COV of IMEP for the High Load Operating Condition (Varying Main DI SOI Timing and CA50)

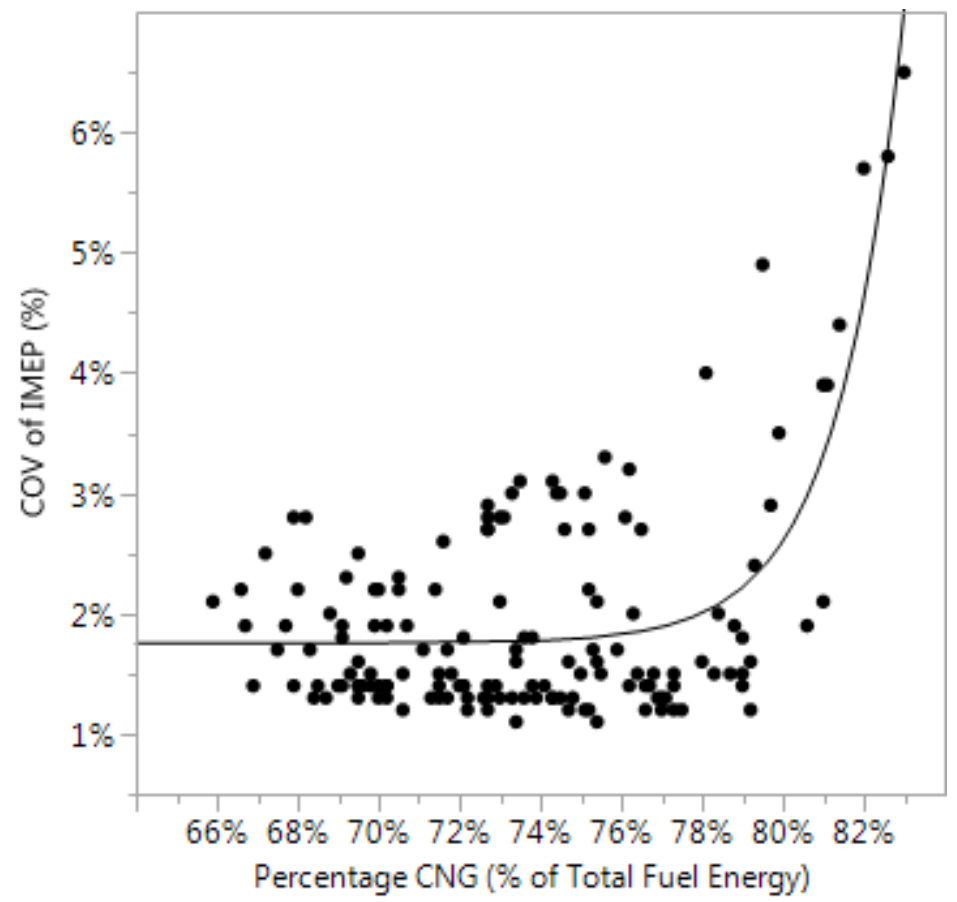

Figure 46: COV of IMEP versus Percentage CNG for the High Load Operating Condition

\subsubsection{Combustion Characteristics Summary}

$\mathrm{CN}$ proved to be influential for nearly all of the combustion characterization metrics discussed in Section

6.2. Maximum in-cylinder PRR for the low and high load operating condition decreased as the $\mathrm{CN}$ of the 
high reactivity fuel increased. This agrees with results from Nieman, et al. [25] and Curran, et al. [27], where increasing the reactivity difference between the low and high reactivity fuels resulted in lower incylinder PRR. At the low load operating condition, there was evidence of AC and T10 influences on maximum in-cylinder pressure and PRR. Lower T10 fuels produced higher in-cylinder pressure, while low and medium CN fuels with low AC resulted in lower in-cylinder PRRs, although this trend did not extend to the high $\mathrm{CN}$ fuels. At the high load operating condition the T10 and AC trends were not apparent with the exception of FACE 1, a low CN and AC fuel continuing to produce lower in-cylinder pressure and PRR than the other low $\mathrm{CN}$ fuels. Maximum HRR decreased as the $\mathrm{CN}$ of the high reactivity fuel increased for both the low and high load operating conditions. This decrease in maximum HRR corresponded to an increase in combustion duration for the higher $\mathrm{CN}$ fuels. T10 was also found to be influential on maximum HRR at both operating conditions, where maximum HRR decreased as the T10 of the high reactivity fuel increased. Subsequently, it was also observed that the combustion duration increased for high reactivity fuels with a higher T10 at the low load operating condition. A similar T10 trend with combustion duration was suggested by a least squares fit model for the high load operating condition although it was not visually apparent.

Contrary to hypothesis 1 , combustion stability (as defined by the COV of IMEP), was worst for the higher $\mathrm{CN}$ high reactivity fuels at the low load operating condition. However, it is important to note that the low CN fuels had to be operated at retarded DI SOI timings to avoid misfire, which certainly would have resulted in very high COV of IMEP values. Furthermore, an exponential correlation between COV of IMEP and percentage of CNG was discovered. High percentage CNG, which was necessary to achieve proper combustion phasing for the high CN fuels, resulted in a higher COV of IMEP. A similar percentage CNG and COV of IMEP trend existed at the high load operating condition, although, on average, the medium and high CN fuels exhibited a lower COV of IMEP than the low CN fuels. With regards to hypothesis 2, the high AC, low CN fuels (FACE 3 and FACE 4), demonstrated lower COV of IMEP, on average, than FACE 1 (low CN and low AC). However, FACE 3 and FACE 4 were much more susceptible to higher in-cylinder PRRs that proved to be more of a limiting factor than COV of IMEP. In fact, as CN of the high reactivity fuel increased, a trend of decreasing in-cylinder PRR was observed for both load conditions, which rejects the notion in hypothesis 2 that a low CN high reactivity fuel will extend high load operation. The opposite was actually observed, the high $\mathrm{CN}$ high reactivity fuels provided the best opportunity for increasing the operable load range of RCCl.

\subsection{Exhaust Emissions}

Exhaust emissions from $\mathrm{RCCl}$ combustion, similar to other advanced combustion strategies, are characteristically low in $\mathrm{NO}_{x}$ and soot, yet high in $\mathrm{CO}$ and THC when compared to CDC. NOx emissions for the low $\mathrm{CN}$ fuels were two to three times higher than the medium and high $\mathrm{CN}$ fuels. Omitting the low CN fuels, at the low load operating condition $\mathrm{NO}_{x}$ emissions were consistently around the 2010 heavy-duty engine emissions standard limit of $0.2 \mathrm{~g} / \mathrm{bhp}$-hr with an average of $0.17 \mathrm{~g} / \mathrm{bhp}-\mathrm{hr}$, while increasing to an average of $0.45 \mathrm{~g} / \mathrm{bhp}-\mathrm{hr}$ at the high load operating condition. Brake specific soot emissions, while varying among the fuels, remained below $10 \mathrm{mg} / \mathrm{bhp}$-hr (except for one test) regardless of the fuel and load, which is the 2010 heavy-duty engine emissions standard for PM. However, measurements of $\mathrm{CO}$ and THC emissions were an order of magnitude higher than values at CDC operation, which is a typical consequence of advanced combustion. It should be noted though, at the high load operating condition $\mathrm{CO}$ emissions measurements for all the high reactivity fuels were less than the heavy-duty engine standard of $15.5 \mathrm{~g} / \mathrm{bhp}$-hr, but at the low load operating condition, the majority 
of the $\mathrm{CO}$ emission measurements were higher than the standard. A comparison of the THC emissions measurements with the standard is not available because the standard is stated in terms of nonmethane hydrocarbons ( $\mathrm{NMHC}$ ) and a separate $\mathrm{CH}_{4}$ emissions measurement was not available for this research. It should also be noted that the results provided herein were for two steady state load points whereas the heavy-duty standard consists of transient and steady state testing and an exact comparison cannot be made.

\subsubsection{NOx Emissions}

It has been demonstrated by previous researchers that $\mathrm{RCCl}$ combustion is capable of producing exceptionally low engine out $\mathrm{NO}_{x}$ emissions, especially compared to $\operatorname{CDC}[16,18,29,31]$. This held true while operating on the medium and high $\mathrm{CN}$ high reactivity fuels in this work. However, $\mathrm{RCCl}$ operation using a low $\mathrm{CN}$ high reactivity fuel produced $\mathrm{NO}_{x}$ emissions that were up to an order of magnitude greater than levels produced by medium and high $\mathrm{CN}$ fuels. One explanation is the reactivity difference between the low $\mathrm{CN}$ fuel and the CNG was less than the other high reactivity fuels, which resulted in less CNG use, higher HRR, and higher in-cylinder PRRs, which all contributed to greater thermal $\mathrm{NO}_{x}$ formation. Additionally, the DI SOI timing utilized for the low CN fuels was retarded compared to the medium and high CN fuels to prevent misfire as detailed in Section 5.3.2. Retarding the DI SOI timing resulted in less time for mixing of the air and fuel, which was evidenced by higher maximum HRRs for $\mathrm{RCCl}$ operation with the low $\mathrm{CN}$ fuels. The higher maximum HRRs for the low $\mathrm{CN}$ fuels, detailed in Section 6.2.2, presumably resulted in higher in-cylinder temperatures and thus higher rates of $\mathrm{NO}_{x}$ formation. Figure 47 displays the disparity in $\mathrm{NO}_{x}$ emissions between the high reactivity fuels at the low load operating condition.

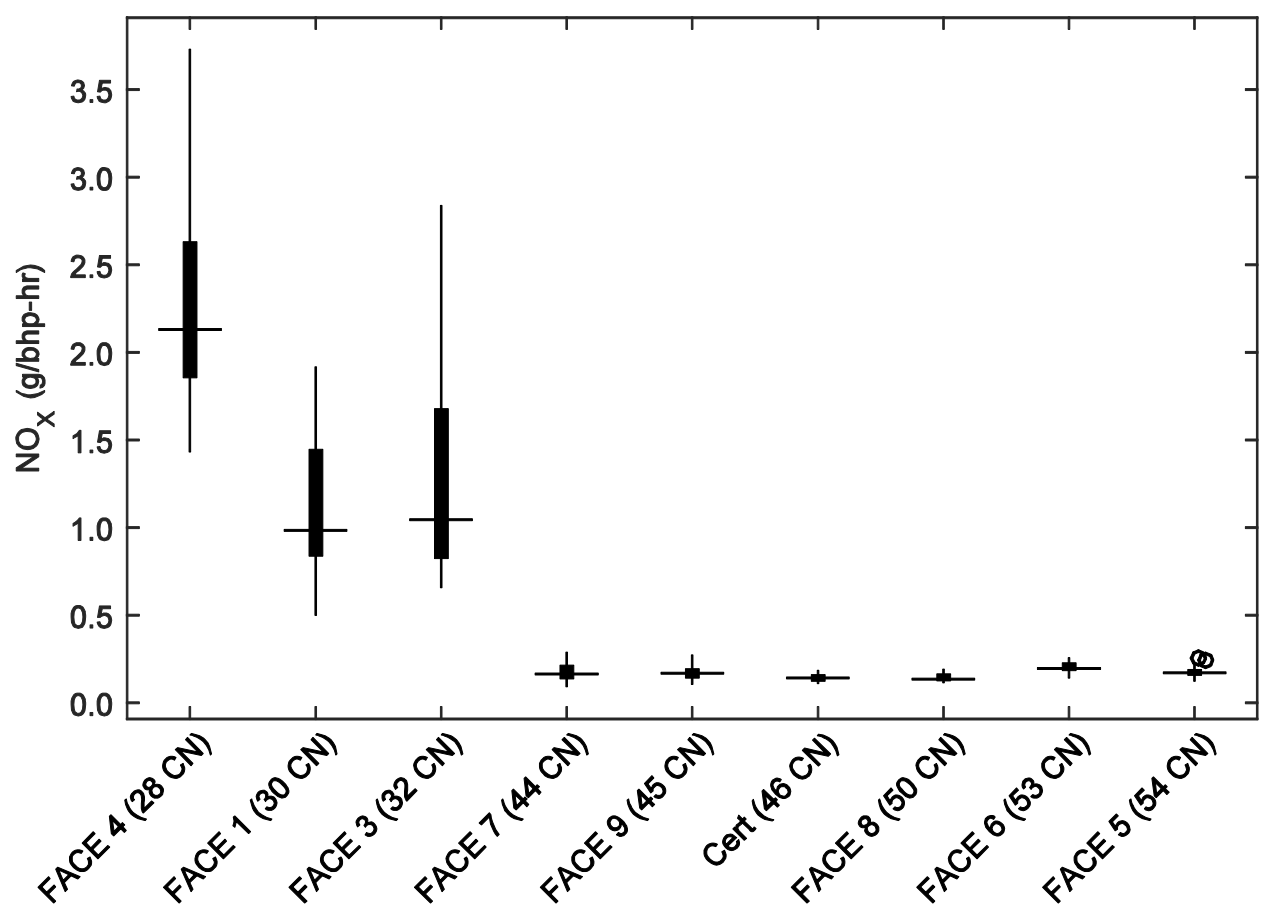

Figure 47: NOx Emissions from the Low Load Operating Condition (Varying IMAP and CA50) 
As noted, there was an obvious threshold of $\mathrm{CN}$ for the high reactivity fuel in which low engine out $\mathrm{NO}_{\mathrm{x}}$ emissions could be achieved. This threshold was directly related to the maximum advancement of DI SOI timing which was dependent on the onset of misfire. Using JMP to explore high reactivity fuel properties effects other than $\mathrm{CN}$ on $\mathrm{RCCl}$ at the low load operating condition suggested a trend of higher $\mathrm{NO}_{x}$ emissions for fuels that had a higher T90 ( $p$-value less than 0.0001). However, upon further scrutiny, confidence in this trend dissipates if the low CN fuels are omitted from the analysis. This is demonstrated in Figure 48, where FACE 7 and FACE 5 are both low T90 fuels, yet neither displays a significant reduction in $\mathrm{NO}_{\mathrm{x}}$ emissions compared to the other high reactivity fuels.

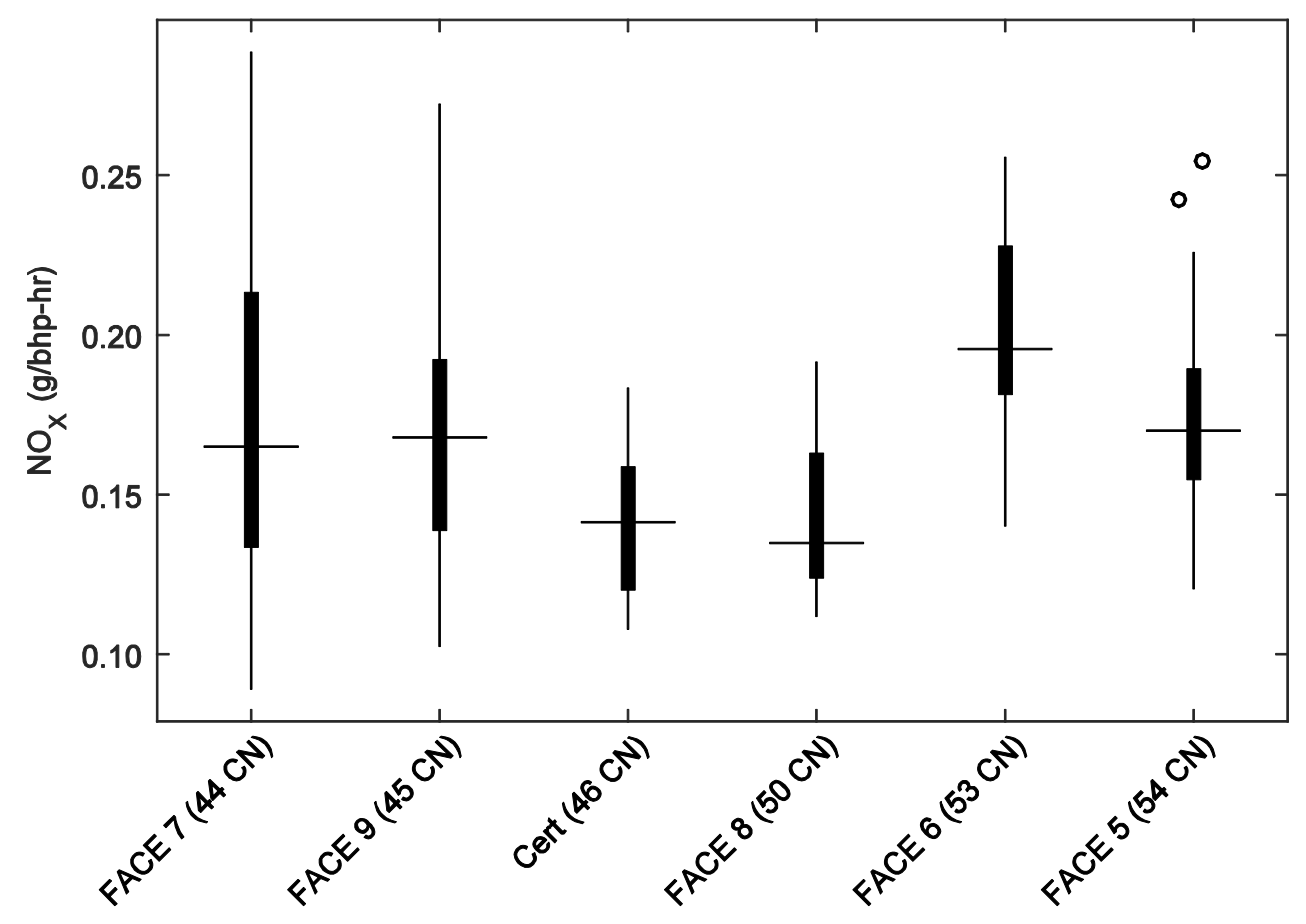

Figure 48: NOx Emissions from the Low Load Operating Condition Omitting Low CN Fuels (Varying IMAP and CA50)

Figure 48 demonstrates that at the low load condition, a high reactivity fuel with at least a CN 4 points greater than the ASTM-D975015c minimum of 40 [52], can achieve BSNO emissions less than $0.2 \mathrm{~g} / \mathrm{bhp}$ $\mathrm{hr}$ regardless of $A C$ and distillation temperature within the range of those tested. At the high load operating condition, low $\mathrm{CN}$ fuels produced exhaust emissions measurements of $\mathrm{NO}_{\mathrm{x}}$ that were, again, significantly higher than the medium and high $\mathrm{CN}$ high reactivity fuels. These results are presented in Figure 49. 


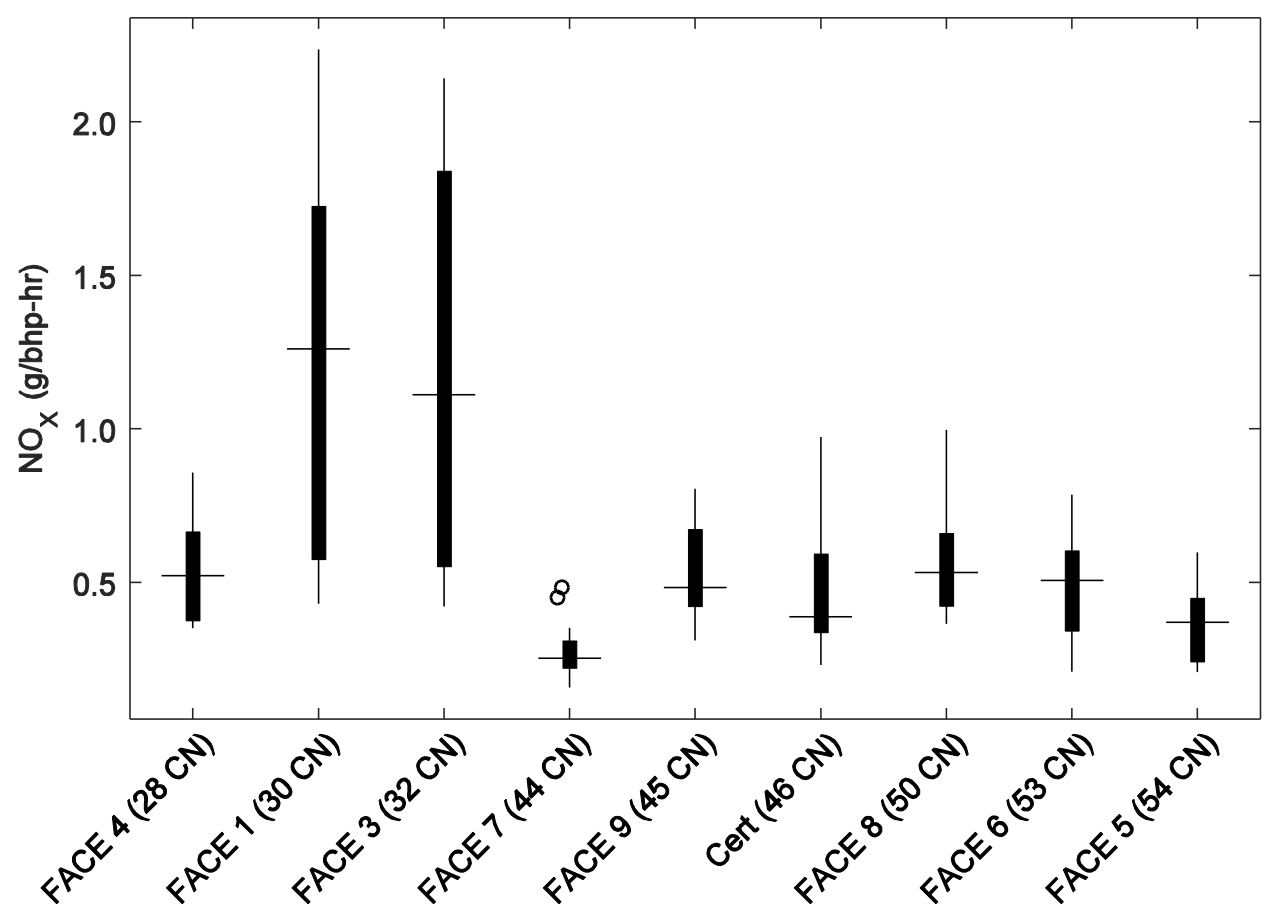

Figure 49: NOx Emissions from the High Load Operating Condition (Varying Main DI SOI Timing and CA50)

As noted in Section 5.3.4, the pilot and main DI SOI timing for the low CN fuels had to be retarded, compared to the pilot and main DI SOI timing used for the medium and high $\mathrm{CN}$ fuels, to prevent misfire. Furthermore, only tests targeting a CA50 of $5 \mathrm{CA}^{\circ}$ ATDC could be performed for FACE 4 due to incylinder PRRs over 10 bar $/ \mathrm{CA}^{\circ}$, which resulted in FACE 4 demonstrating what appeared to be lower overall $\mathrm{NO}_{x}$ emissions compared to the other low $\mathrm{CN}$ fuels. Although there were instances, where $\mathrm{RCCl}$ with the low $\mathrm{CN}$ fuels produced $\mathrm{NO}_{x}$ emissions similar to the medium and high $\mathrm{CN}$ fuels, the range of NOx emissions measured were much higher for FACE 1 and FACE 3. It was found that NOx emissions from these fuels were much more susceptible to engine control parameter changes, namely main DI SOI timing and percentage CNG. Disregarding the low $\mathrm{CN}$ fuels, a trend of decreasing $\mathrm{NO}_{\mathrm{x}}$ emissions for high reactivity fuels with lower T90 values is illustrated in Figure 50. 


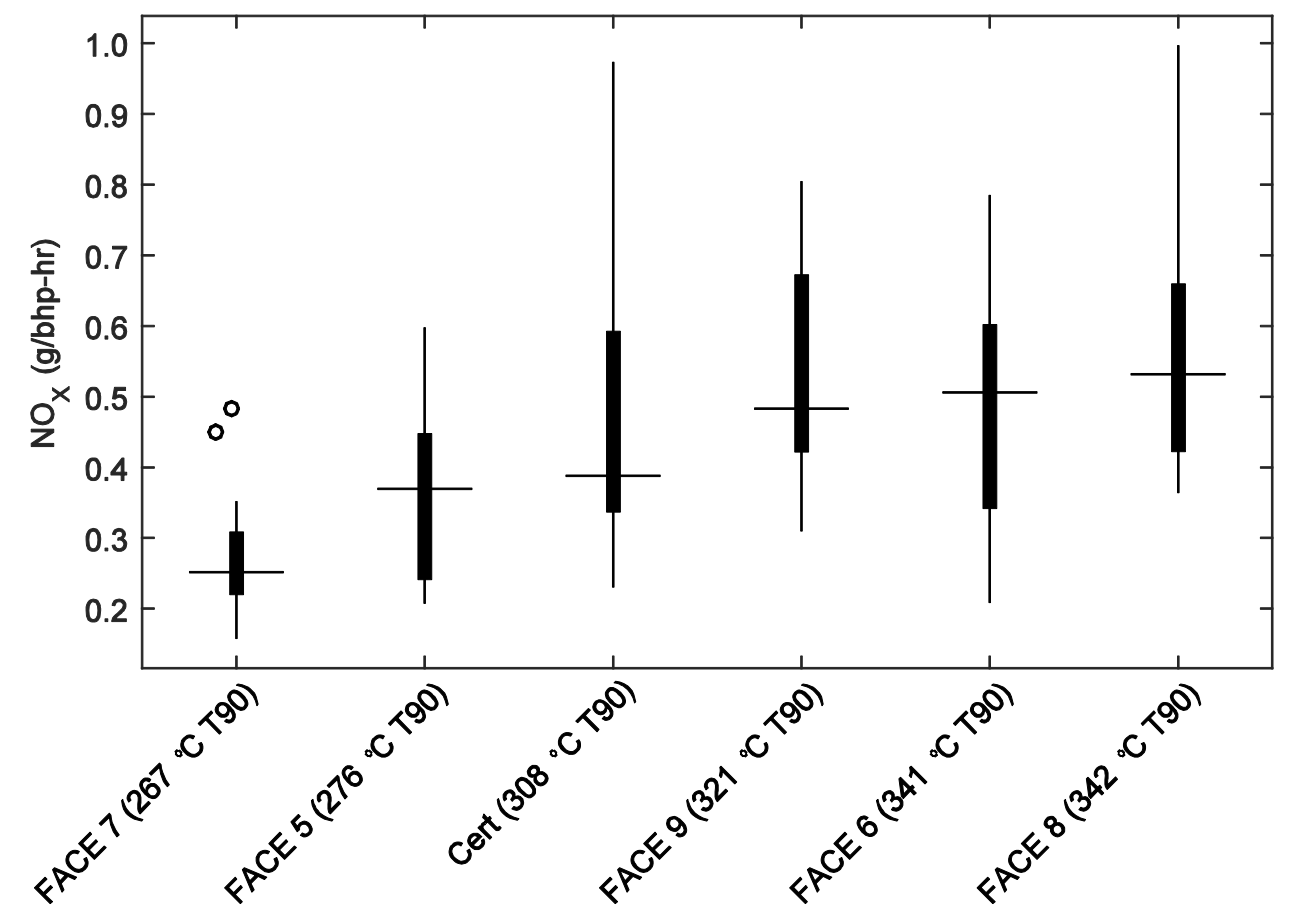

Figure 50: NOx Emissions from the High Load Operating Condition Ordered by T90 Omitting Low CN Fuels (Varying Main DI SOI Timing and CA50)

It is clearly demonstrated by Figure 50 that when considering the medium and high $\mathrm{CN}$ fuels at the high load operating condition there was a correlation between $\mathrm{NO}_{\mathrm{x}}$ emissions and T90. Examining the medians (horizontal lines) for each fuel, $\mathrm{NO}_{\mathrm{x}}$ emissions increase as the T90 of the high reactivity fuel increases. A least squares fit model suggested a high level of confidence in the influence of T90 and CA50 ( $p$-values less than 0.0001 ), but no other parameters. Additionally, the intercept of the equation had a high $p$-value. The model exhibited an $R^{2}$ of 0.56 and is described by Equation 46.

\section{Equation 46: Least Squares Fit Model for $\mathrm{NO}_{x}$ Emissions for the Medium and High CN Fuels at the High Load Operating Condition}

$$
B S N O_{X}=-0.082813-0.096568 * C A 50+3.1006 * 10^{-3} * T 90
$$

Higher peaks of $\mathrm{NO}_{\mathrm{x}}$ emissions were observed for the Cert and FACE 8. Noting that CA50 was influential based on Equation 46, further investigation revealed that at the most advanced CA50 target, $3 \mathrm{CA}^{\circ}$ ATDC, $\mathrm{RCCl}$ operation at this load with these fuels was more susceptible to high $\mathrm{NO}_{\mathrm{x}}$ formation than with the other fuels presented in Figure 50; however, no correlation to fuel properties could be made.

\subsubsection{Soot Emissions}

Exhaust measurements of soot were very low regardless of the high reactivity fuel used. In fact, there was only one instance in which a brake specific soot measurement was over $10 \mathrm{mg} / \mathrm{bhp}$-hr. Furthermore, that measurement was only $10.02 \mathrm{mg} / \mathrm{bhp}$-hr from FACE 8 at the high load operating condition and was considered an outlier. FACE 8 also exhibited the highest brake specific soot measurement at the low load operating condition, which was $6.97 \mathrm{mg} / \mathrm{bhp}-\mathrm{hr}$. 


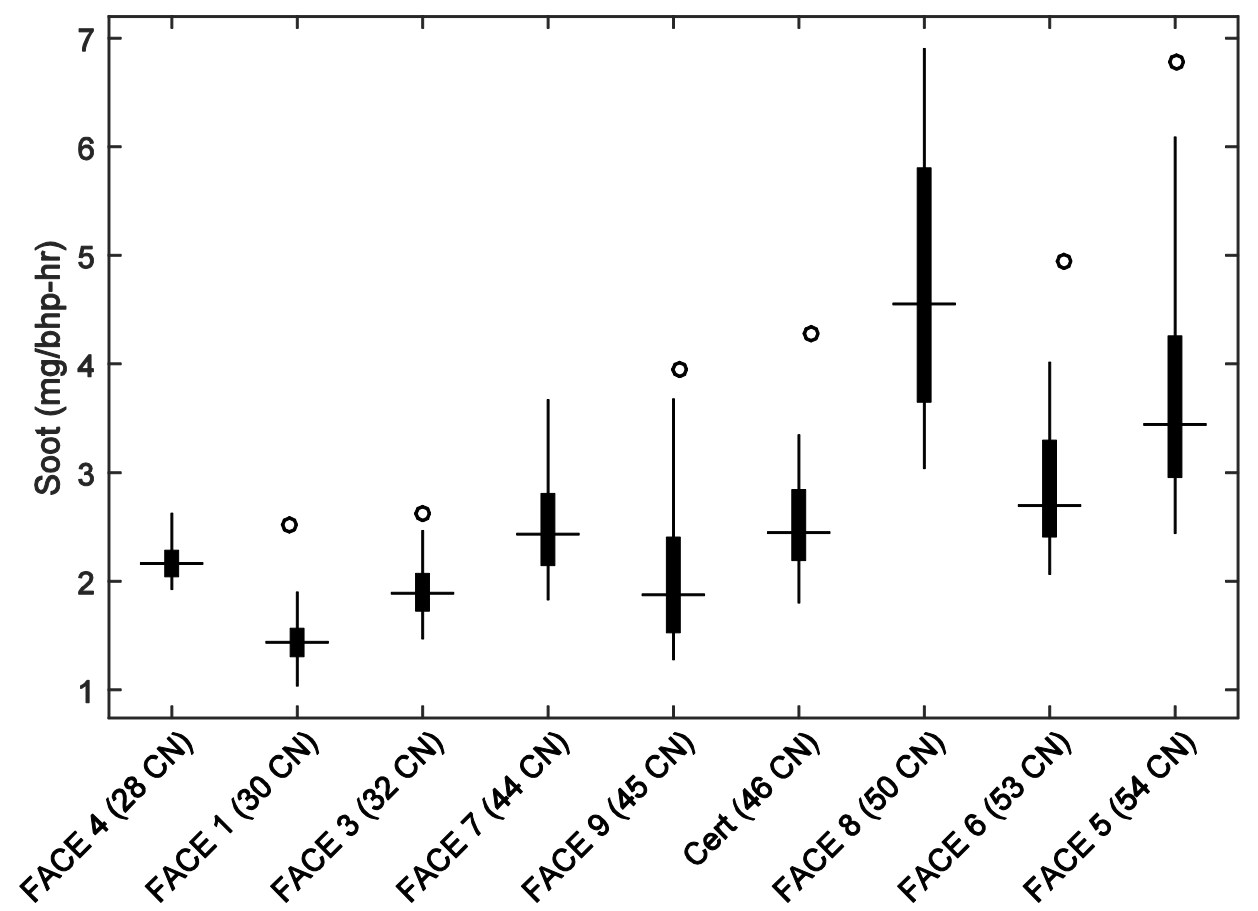

Figure 51: Soot Emissions from the Low Load Operating Condition (Varying IMAP and CA50)

During CDC and LTC, high CN fuels typically produce more soot than their lower CN counterparts due to their reduced ignition delay, which results in less time for mixing and more locally rich regions during combustion. At the low load operating condition, soot emissions, although very low, were noticeably higher for the high CN fuels compared to the low CN fuels demonstrated by Figure 51. A screening analysis and subsequent least squares fit model also suggested that other fuel properties were influential. The model is defined by Equation 47 and exhibited an $R^{2}$ value of 0.64 .

\section{Equation 47: Least Squares Fit Model for Soot Emissions at the Low Load Operating Condition}

$$
\begin{gathered}
\text { BSSoot }=4.2535+0.13795 * C N+1.2751 * 10^{-3} *(T 10-198.14)^{2}-0.17844 * \text { DI SOI }-4.1317 \\
* 10^{-3} *(T 10-198.14) *(C N-43.003)+0.017120 * A C
\end{gathered}
$$

The suggested trend with AC can be observed in Figure 51 for the low CN fuels where FACE 1 (low AC) produced less soot on average than FACE 4 and FACE 3 (high AC). It can also be observed for the high CN fuels where FACE 8 (high AC) produced more soot than FACE 6 and FACE 5 (low AC). However the trend is not present for the medium CN fuels, the Cert fuel (low AC) did not produce lower soot than FACE 7 and FACE 9 (high AC). This is potentially related to the higher T10 of the Cert fuel versus the other medium CN fuels. Equation 47 suggests that T10 is influential on soot production at the low load operating condition and Figure 52 provides visual confirmation of the influence of T10. 


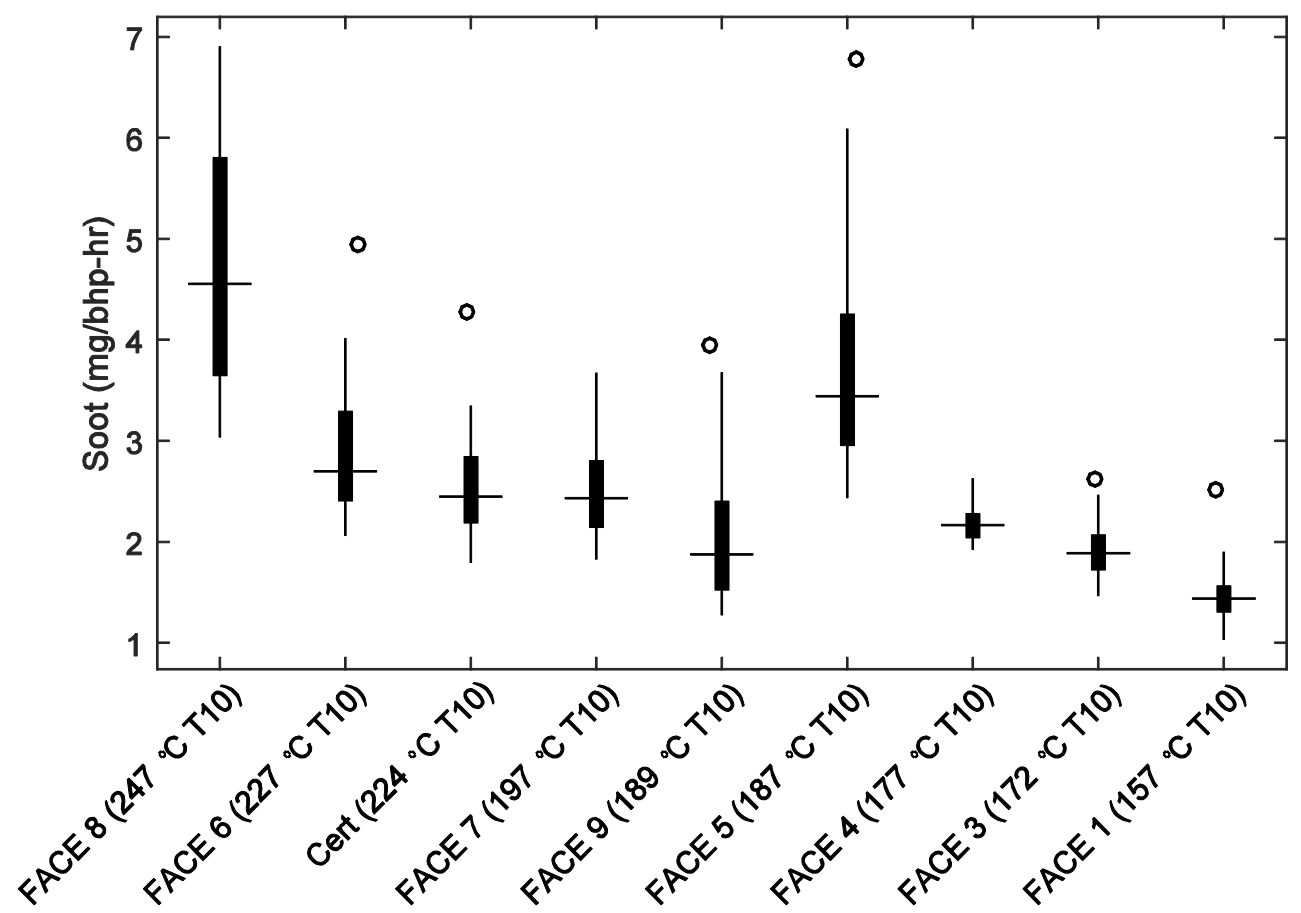

Figure 52: Soot Emissions from the Low Load Operating Condition Ordered by T10 (Varying IMAP and CA50)

For other advanced combustion regimes that use a single fuel, T90 has been correlated to soot production [14, 35]. This is based on the notion that the heaviest components of the diesel fuel (highest carbon content, such as aromatic compounds) are the last to vaporize and are also the largest contributors of soot. The influence of T10 on soot, rather than T90 for RCCI may be related to the use of two fuels. A high reactivity fuel with a lower T10 will vaporize and begin to mix with the low reactivity fuel sooner providing a more homogenized air and fuel mixture. The lower carbon intensity of the low reactivity fuel, CNG in this case, compared to diesel fuel also provides lower soot production and may alter the typical fuel property trends observed with soot production from neat diesel fuel combustion. 


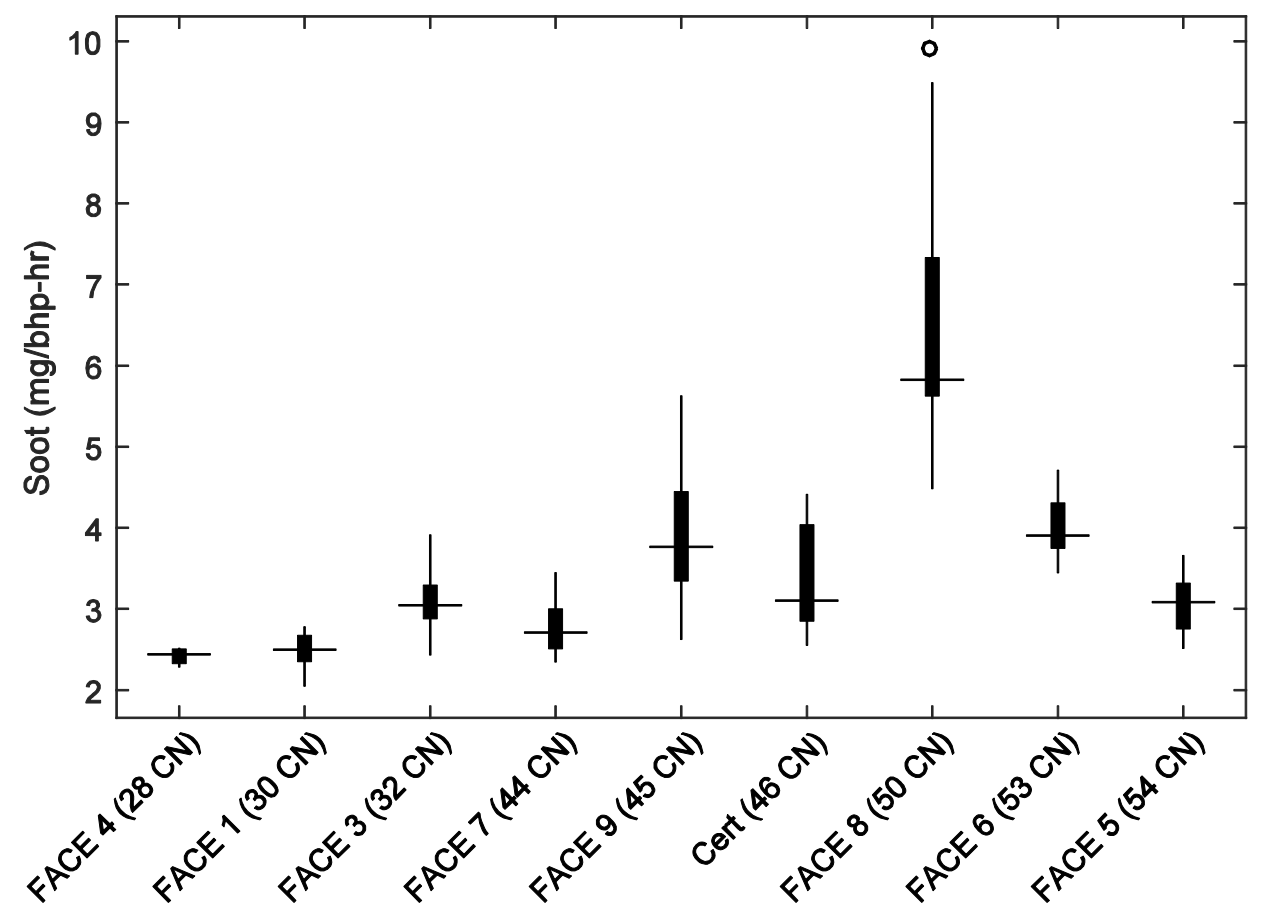

Figure 53: Soot Emissions from the High Load Operating Condition (Varying Main DI SOI Timing and CA50)

Results at the high load operating condition presented in Figure 53 did not produce as clear of a $\mathrm{CN}$ trend as the low load operating condition. A least squares fit model developed for soot emissions at the high load operating condition suggested that T10 and AC were influential with a high level of confidence ( $p$ value less than 0.0001 ). Note that $\mathrm{CN}$ exhibited a $\mathrm{p}$ value of 0.0002 , which is still very low and suggested confidence in its influence. The model, displayed by Equation 48 , had an $\mathrm{R}^{2}$ value of 0.70 .

\section{Equation 48: Least Squares Fit Model for Soot Emissions at the High Load Operating Condition}

$$
\begin{gathered}
\text { BSSoot }=-2.9411+7.7512 * 10^{-4} *(T 10-198.84)^{2}+0.034991 * A C+0.018846 * T 10 \\
-0.25492 * C A 50+0.050207 * C N
\end{gathered}
$$

The influence of T10 was not as visually apparent for the high load operating condition as it was for the low load operating condition as displayed by Figure 54 . It is important to reiterate that, regardless of fuel properties, nearly all of the soot measurements obtained during $\mathrm{RCCl}$ operation were less than 10 $\mathrm{mg} / \mathrm{bhp}-\mathrm{hr}$. It can be concluded that although the trends were not absolute and necessarily linear in nature, high reactivity fuels with a higher $\mathrm{CN}$ and $\mathrm{T} 10$ values have a greater propensity to produce soot during $\mathrm{RCCl}$ combustion. 


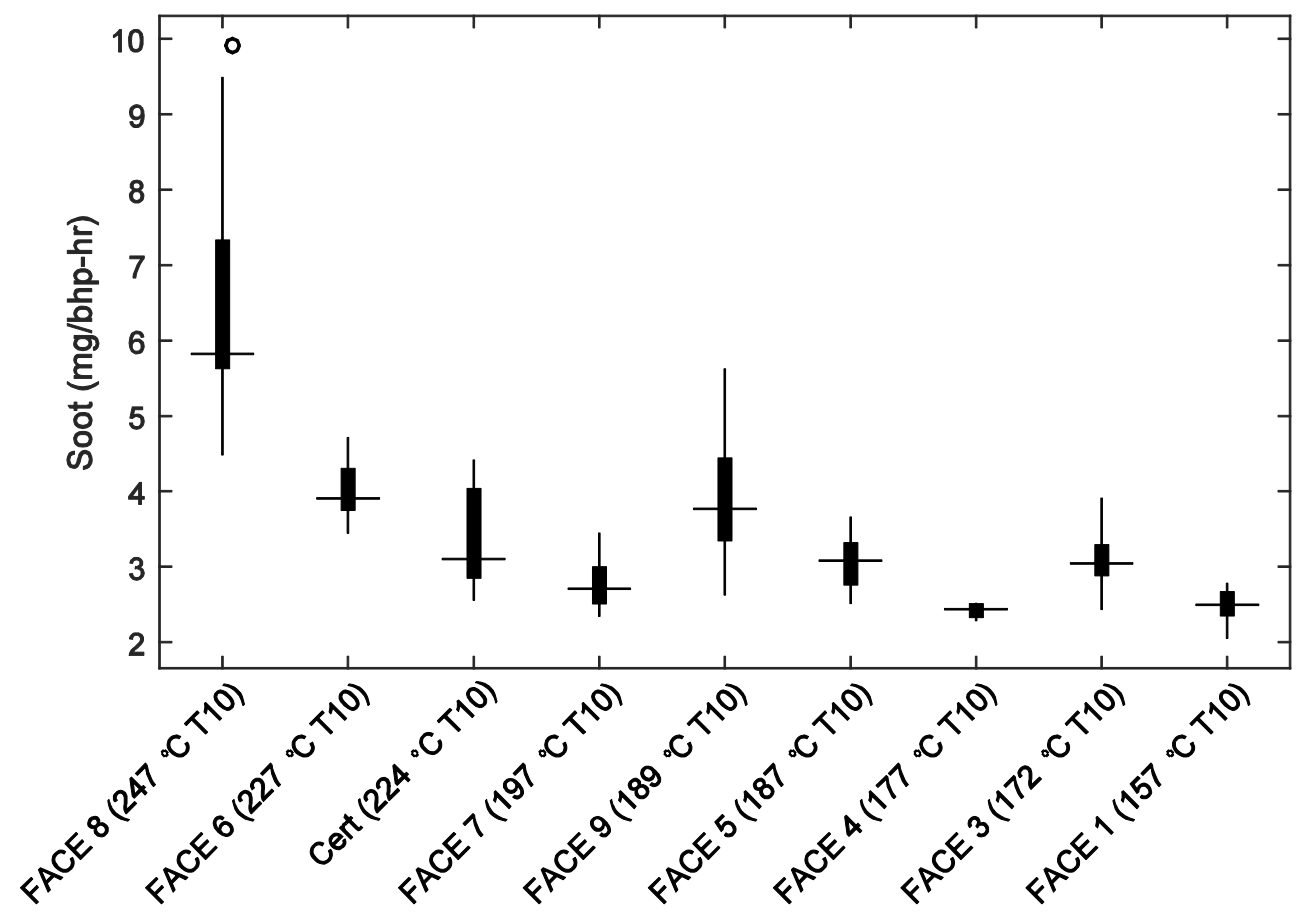

Figure 54: Soot Emissions from the High Load Operating Condition Ordered by T10 (Varying Main DI SOI Timing and CA50)

\subsubsection{CO and THC Emissions}

Advanced combustion regimes decouple the conventional $\mathrm{NO}_{\mathrm{x}}$ versus soot emissions tradeoff observed for CDC but typically result in elevated $\mathrm{CO}$ and THC emissions. This is a result of the LTC that reduces thermal $\mathrm{NO}_{\mathrm{x}}$ formation, but decreases the oxidation of $\mathrm{CO}$ and THC. Furthermore, advanced combustion strategies generally allow more time for mixing of the fuel in the cylinder, which provides enhanced opportunity for fuel to locate itself near the cylinder walls, and in crevices such as the piston ring lands outside of the combustion area. $\mathrm{CO}$ and $\mathrm{THC}$ emissions from $\mathrm{RCCl}$ were typically an order of magnitude greater than $\mathrm{CO}$ and THC emissions from CDC. For example, at the low load operating condition, FACE 5 exhibited an average of $1.61 \mathrm{~g} / \mathrm{bhp}$-hr and $0.573 \mathrm{~g} / \mathrm{bhp}-\mathrm{hr}$ of CO and THC emissions, respectively, during CDC. These figures rose by approximately 1100 percent and 5100 percent to 17.7 $\mathrm{g} / \mathrm{bhp}$-hr and $29.4 \mathrm{~g} / \mathrm{bhp}$-hr for CO and THC emissions, respectively. This increase in CO and THC emissions, as well as low exhaust temperatures from LTC may present difficulties for conventional DOCs as noted by Prikhodko, et al. [24]. It is possible that changes in catalysts materials, higher fuel consumption, or active heating may be necessary to effectively oxidize $\mathrm{CO}$ and $\mathrm{THC}$ emissions from $\mathrm{RCCl}$ combustion. Figure 55 and Figure 56 present these elevated $\mathrm{CO}$ and THC emissions, respectively, for the low load operating condition. 


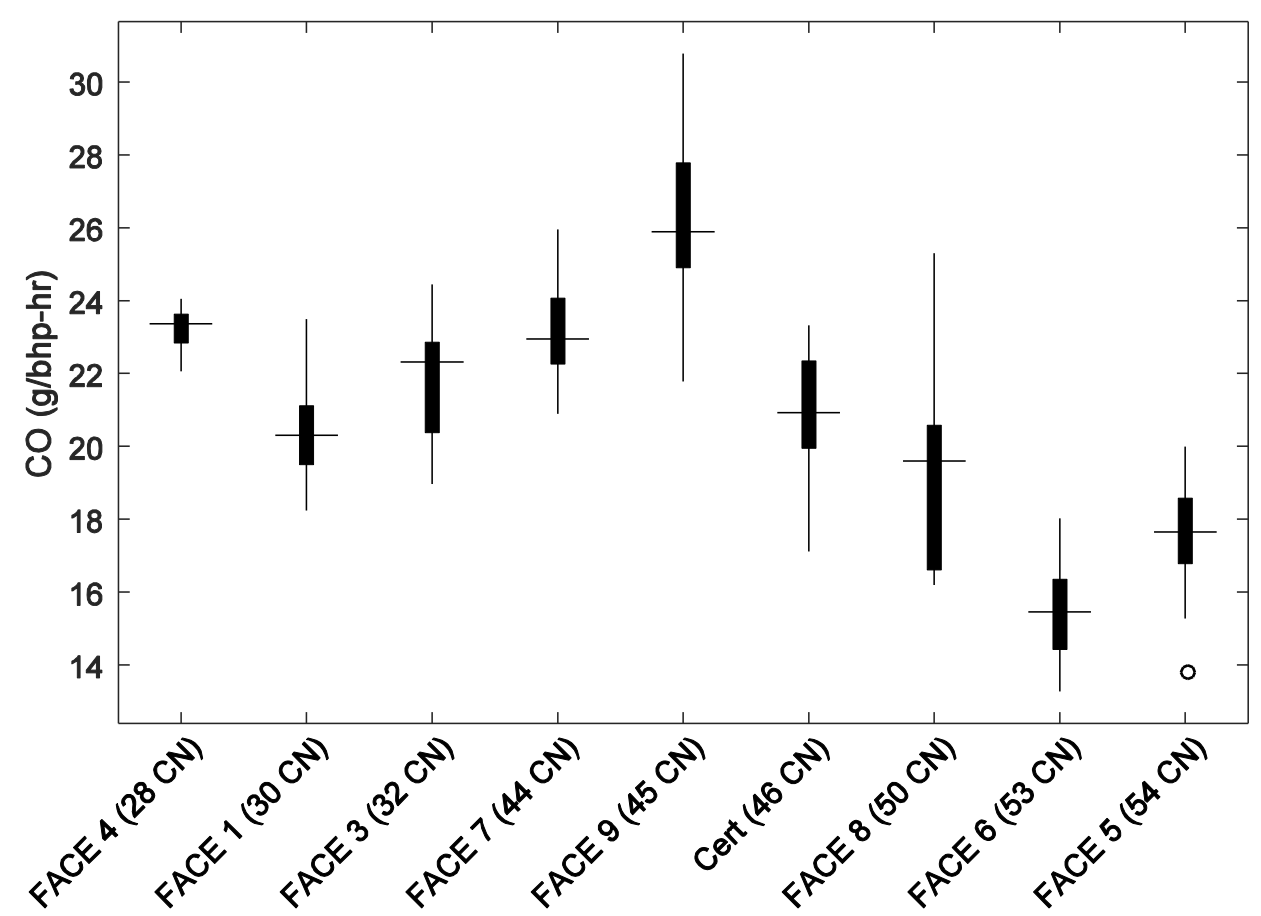

Figure 55: CO Emissions from the Low Load Operating Condition (Varying IMAP and CA50)

Figure 55 demonstrates that $\mathrm{RCCl}$ combustion with the high $\mathrm{CN}$ fuels generally produced less $\mathrm{CO}$ compared to the other fuels. In fact, a linear trend with CN was expected, however, because the DI SOI timing had to be retarded to prevent misfire for the low $\mathrm{CN}$ fuels, this resulted in a tradeoff of substantially higher $\mathrm{NO}_{x}$ emissions (Figure 47) for $\mathrm{CO}$ emissions that were on par with the medium $\mathrm{CN}$ fuels. Visually examining Figure 55 for other fuel property effects while considering $\mathrm{CN}$ and DI SOI timing revealed a potential $A C$ effect on $\mathrm{CO}$ emissions. In their respective $\mathrm{CN}$ groups, high reactivity fuels with a low AC (FACE 1, Cert, FACE 6 and FACE 5) all produce, on average, less CO than their high aromatic counterparts did. Aromatic compounds have a higher carbon-to-hydrogen ratio compared to other diesel fuel components, and thus a higher propensity to form CO. These observations are supported by a least squares fit model, which included CN, AC, DI SOI timing, CA50 and a CN multiplied by DI SOI timing term. The model is defined by Equation 49, which had an $\mathrm{R}^{2}$ of 0.66 .

Equation 49: Least Squares Fit Model for CO Emissions at the Low Load Operating Condition

$$
\begin{gathered}
B S C O=-54.356-0.93306 * C N+2.2031 * D I S O I+0.093826 * A C+1.2918 * C A 50+0.10540 \\
*(C N-43.003) *(D I S O I-46.489)
\end{gathered}
$$

The inclusion of DI SOI and the CN multiplied by DI SOI term in Equation 49 supports the presumption that retarding the DI SOI timing reduced the $\mathrm{CO}$ emissions for the low $\mathrm{CN}$ fuels. 


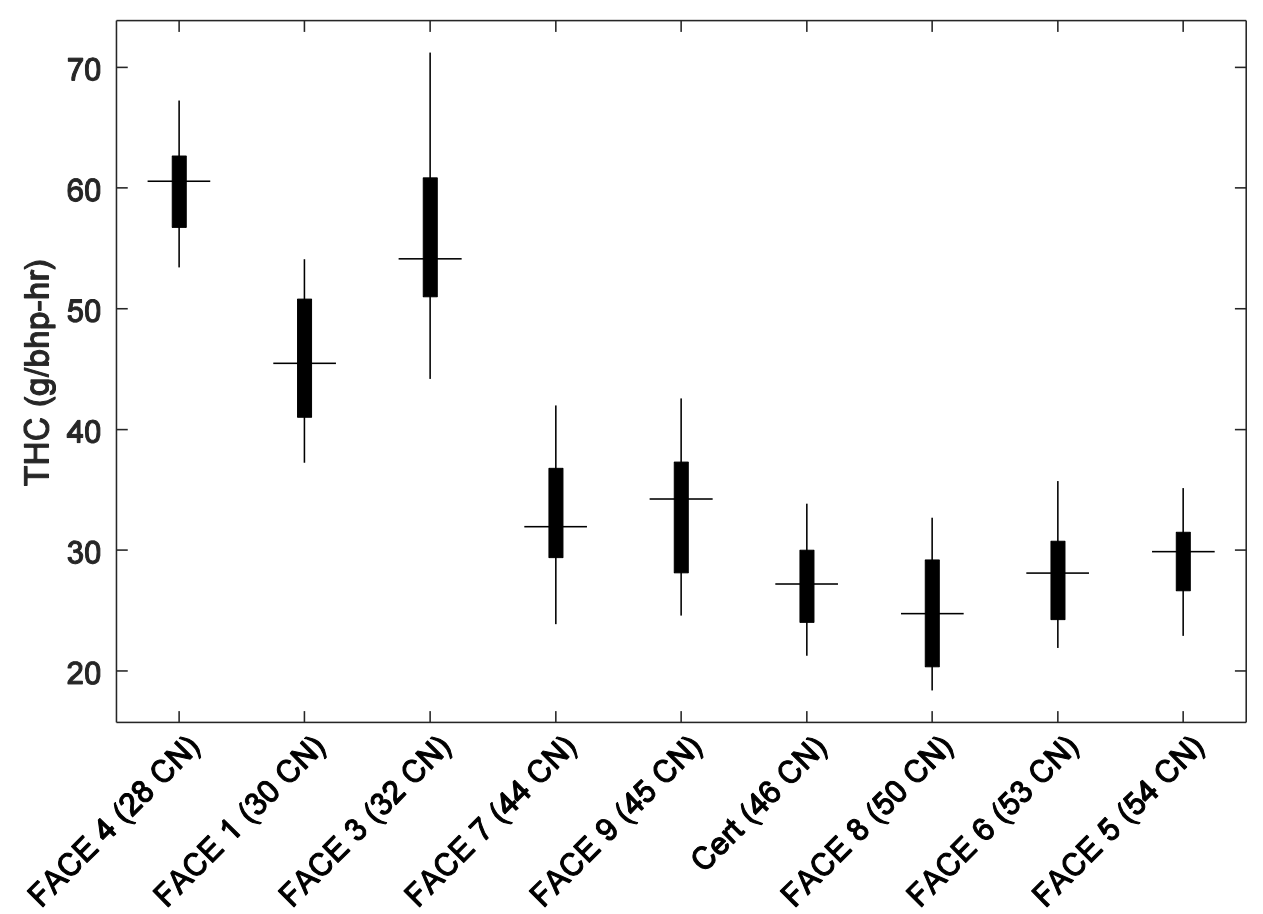

Figure 56: THC Emissions from the Low Load Operating Condition (Varying IMAP and CA50)

Regardless of the changes in DI SOI timing for the low CN high reactivity fuels, THC emissions followed a decreasing trend as a higher $\mathrm{CN}$ high reactivity fuel was used. There are deviations from this trend, as shown in Figure 56, where FACE 1 exhibits lower THC emissions compared to the other low CN fuels, while FACE 6 and FACE 5 exhibit slightly higher THC emissions than the other high CN fuel, FACE 8. One explanation can be found in the percentage CNG presented by Figure 24 . It was hypothesized that the THC emissions were predominantly composed of the low reactivity fuel, CNG, based on its ratio in the total fuel mixture and the opportunity for it to reach crevices since it was injected into the intake port and reached the cylinder earlier than the high reactivity fuel. Furthermore, FACE 1 required the lowest percentage CNG, on average amongst the low CN fuels, and FACE 5 and FACE 6 required a higher percentage CNG, on average than FACE 8 . These observations are supported by a least squares fit model described by Equation 50, which only includes CN and Percentage CNG, yet had a relatively high $R^{2}$ value of 0.87 .

\section{Equation 50: Least Squares Fit Model for THC Emissions at the Low Load Operating Condition}

$$
B S T H C=-49.554-1.8025 * C N+0.023082 * \text { Percentage } C N G
$$

Another explanation for the deviation from the trend of decreasing THC emissions with increasing $\mathrm{CN}$ can be found by investigating trends for the medium and high $\mathrm{CN}$ high reactivity fuels. Equation 51 describes a least squares fit model $\left(R^{2}\right.$ of 0.75$)$ that suggested T10 was influential with a high level of confidence for the medium and high $\mathrm{CN}$ fuels. Figure 57 provides a visualization of the trend. 


\section{Equation 51: Least Squares Fit Model for THC Emissions from Medium and High CN Fuels at the Low Load Operating Condition}

$$
\begin{gathered}
B S T H C=25.683+4.5422 * C A 50-0.12296 * T 10+2.1597 * I M A P+0.063824 \\
*(T 10-211.83) *(I M A P-5.5105)
\end{gathered}
$$

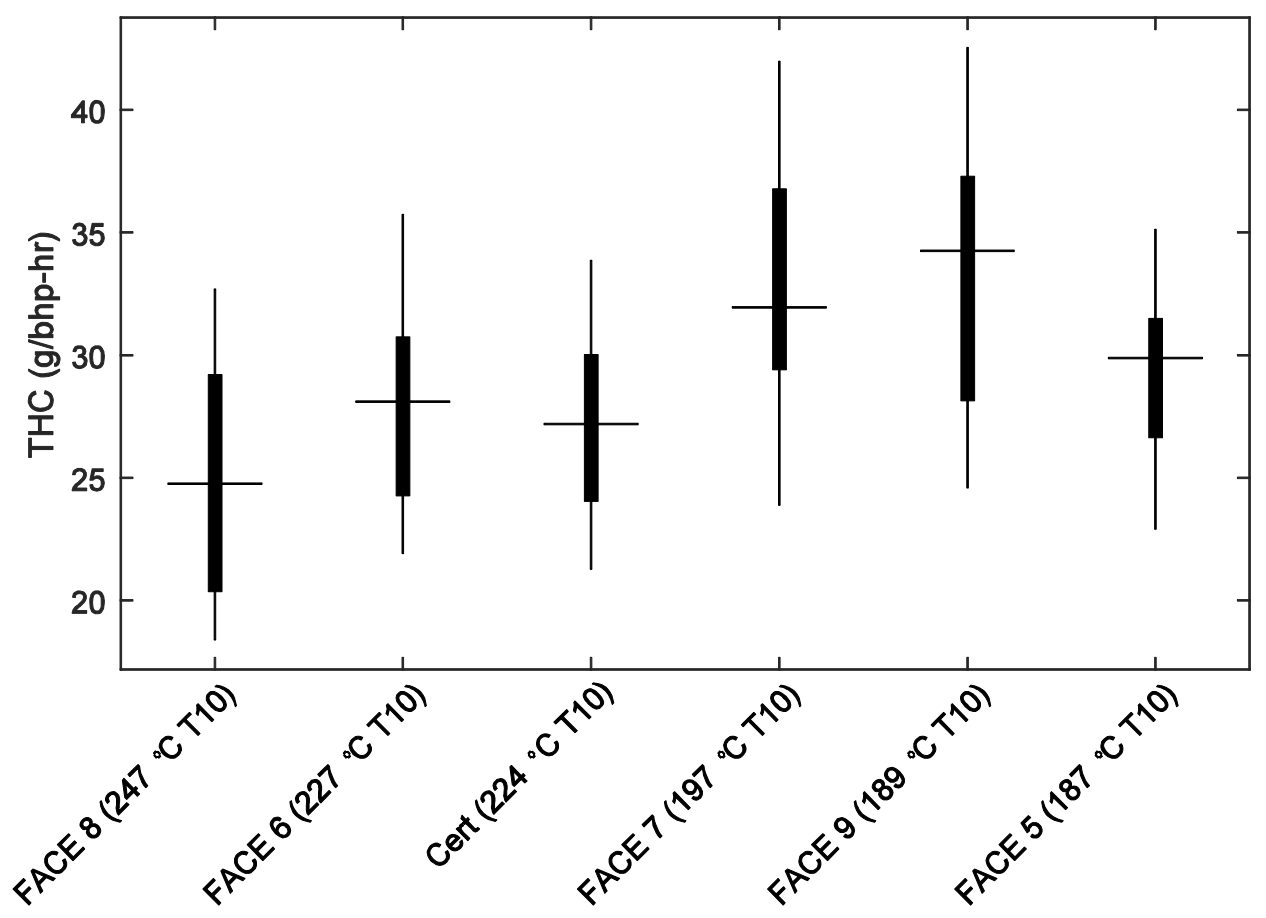

Figure 57: THC Emissions from the Low Load Operating Condition Ordered by T10 Omitting Low CN Fuels (Varying IMAP and CA50)

Figure 57 provides evidence of a decreasing THC emissions trend with increasing T10 at the low load operating condition. Considering $\mathrm{CN}$ and its dominant effects on THC emissions, the T10 trend is easily identifiable for the medium CN fuels (Cert, FACE 7, and FACE 9), but there is also a subtle effect for FACE 8, FACE 6 and FACE 5.

At the high load operating condition, $\mathrm{RCCl}$ operation with the medium and high $\mathrm{CN}$ high reactivity fuels resulted in lower, on average, $\mathrm{CO}$ and THC emissions demonstrated by Figure 58 and Figure 59, respectively. Similar to the low load operating condition, the DI SOI timing for the low CN fuels had to be retarded to prevent misfire. This included the main DI injection and the pilot DI injection, since a dual injection strategy was used for this load to reduce $\mathrm{NO}_{\mathrm{x}}$ emissions and in-cylinder PRR, as detailed in Section 5.3.3. Despite the dual injection approach, FACE 4 could only be tested at CA50 targets of $5 \mathrm{CA}^{\circ}$ ATDC because of in-cylinder PRRs over 10 bar $/ C A^{\circ}$. Given the DI SOI timing changes made to the low $\mathrm{CN}$ fuels to prevent incomplete combustion, the $\mathrm{CO}$ emissions during operation with these fuels remained, on average, greater than the higher $\mathrm{CN}$ fuels, unlike the low load operating condition. 


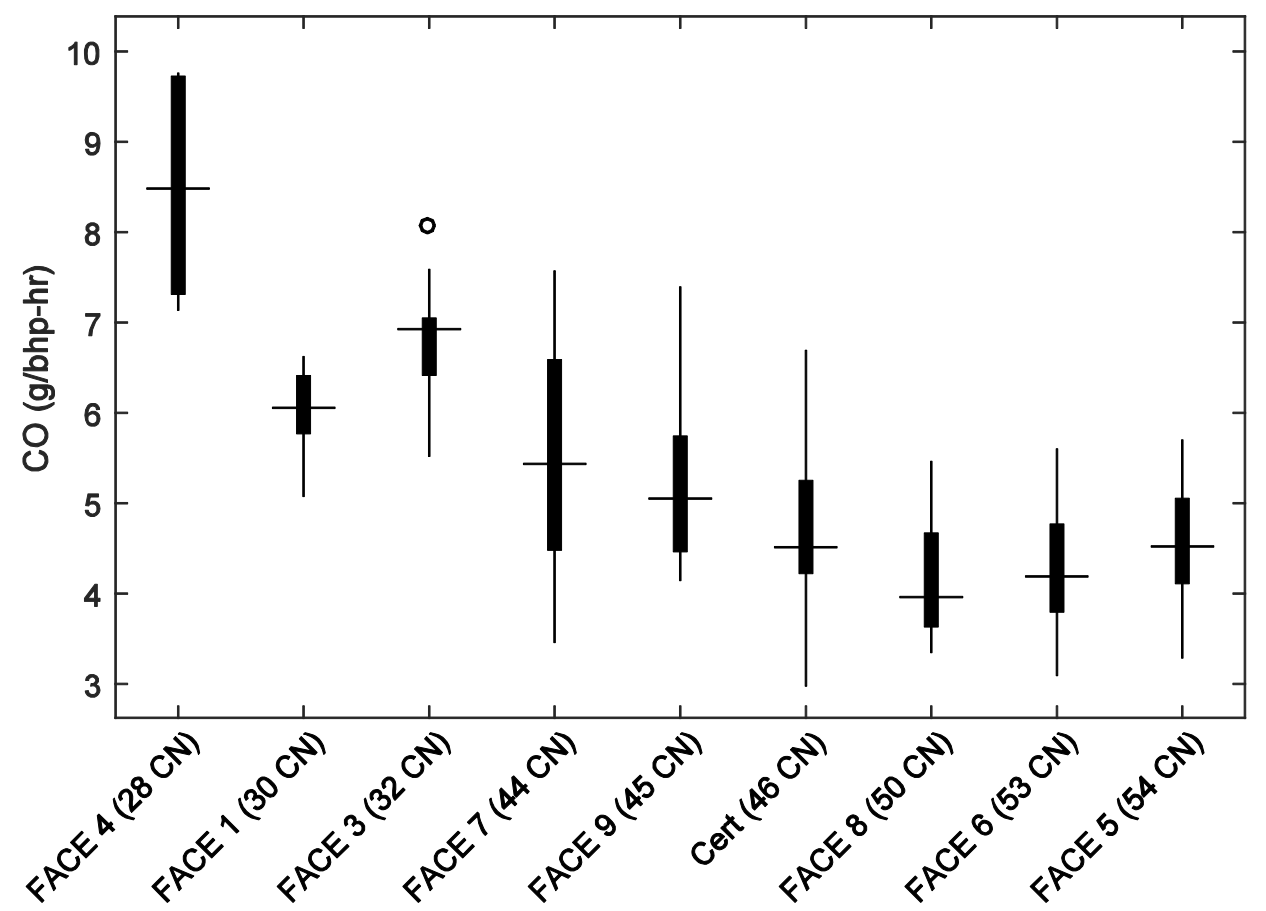

Figure 58: CO Emissions from the High Load Operating Condition (Varying Main DI SOI Timing and CA50)

Similar to the low load operating condition, Figure 58 suggests a possible correlation with $\mathrm{AC}$ in addition to $\mathrm{CN}$, which was confirmed by a least squares fit analysis performed with JMP. The least squares fit model described by Equation 52 ( $R^{2}$ of 0.76 ), suggested that besides $C N$ and $C A 50$, the product of $A C$ and $\mathrm{CN}$ was influential on $\mathrm{CO}$ emissions at this operating condition with a high level of confidence.

Equation 52: Least Squares Fit Model for CO Emissions at the High Load Operating Condition

$$
\begin{gathered}
B S C O=6.9025-0.098923 * C N+0.58595 * C A 50-3.9422 * 10^{-3} *(C N-43.639) \\
*(A C-32.557)
\end{gathered}
$$

Note in Figure 58 that FACE 1 (low AC) produced lower CO emissions than the other low CN fuels, FACE 4 and FACE 3 (high AC). Similarly, for the medium CN fuels, FACE 7 and FACE 9 (high AC) produced higher $\mathrm{CO}$ emissions than the Cert fuel (low $\mathrm{AC}$ ). However, the trend does not carry over to the high $\mathrm{CN}$ fuels, which explains the inclusion of the AC multiplied by CN term. FACE 8 (high AC) produced lower, on average, CO emissions than FACE 6 and FACE 5 (low AC). The deviation from the AC trend for the high CN fuels may be related to the higher percentage CNG for FACE 6 and FACE 5 compared to FACE 8, which is presented in Figure 25. In fact, FACE 8 required an average percentage CNG of 72.9 percent to maintain combustion phasing while, FACE 6 and FACE 5 required 78.8 percent and 78.1 percent, respectively. The effect of higher percentage CNG for FACE 6 and FACE 5 was also visible in greater THC emissions for these fuels compared to FACE 8, which is displayed in Figure 59. 


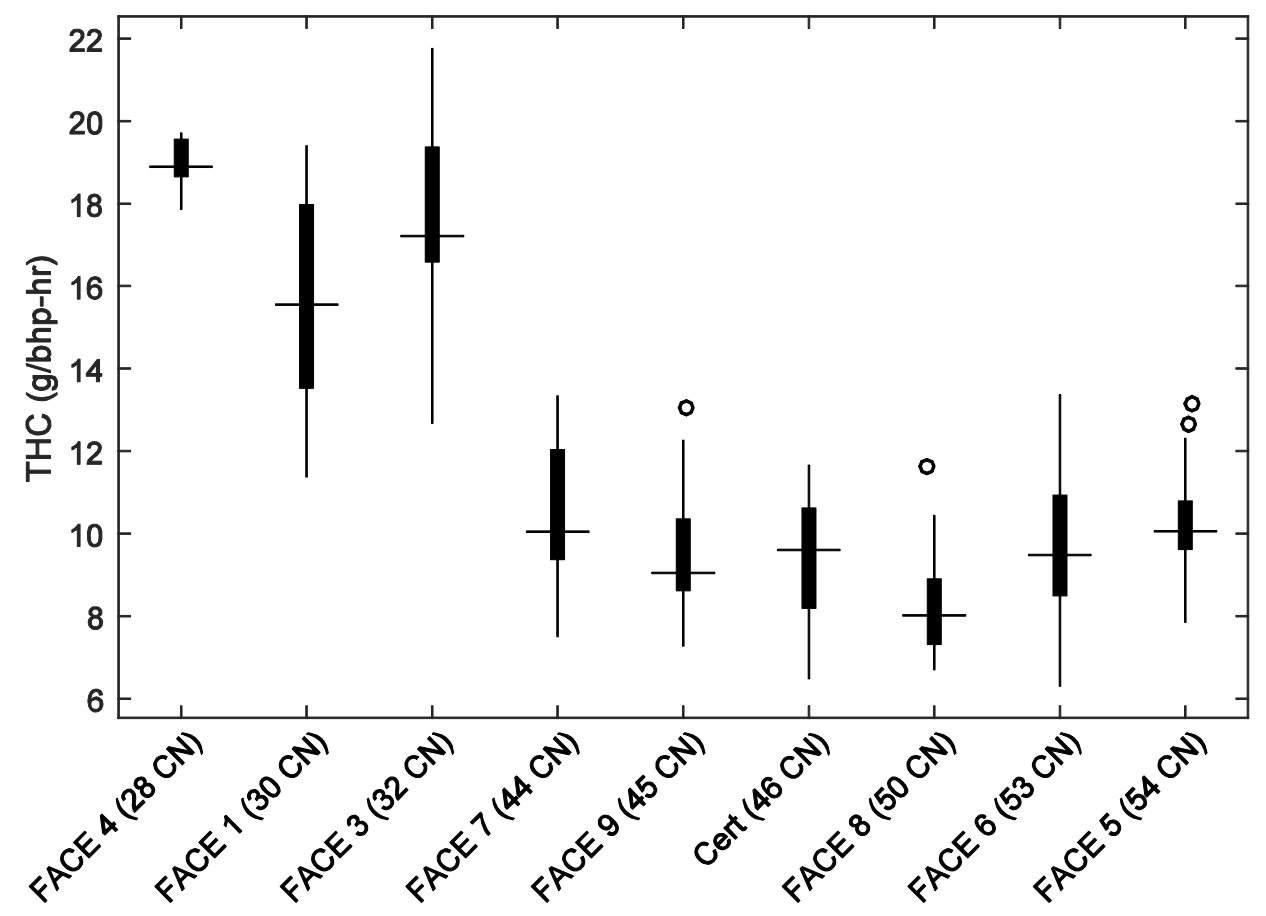

Figure 59: THC Emissions from the High Load Operating Condition (Varying Main DI SOI Timing and CA50)

In accordance with the low load operating condition, THC emissions at the high load operating condition were notably higher for the low CN fuels. As eluded to previously, percentage CNG correlates with THC emissions from the medium and high $\mathrm{CN}$ high reactivity fuels. FACE 7 and the Cert fuel required a higher percentage of CNG, on average, to maintain combustion phasing than the other medium CN fuel, FACE 9, and subsequently produced greater THC emissions on average. Similar for the high CN fuels, FACE 6 and FACE 5 required higher percentage CNG and thus produced greater THC emissions compared to FACE 8.

\subsubsection{Exhaust Emissions Summary}

$\mathrm{CN}$ was the most influential parameter on $\mathrm{RCCl}$ combustion characteristics, and as a result, it had overarching effects on exhaust emissions compounds. At both load conditions, the low $\mathrm{CN}$ high reactivity fuels could not operate at a DI SOI timing that was advanced enough to prevent excessive $\mathrm{NO}_{x}$ emissions. $\mathrm{RCCl}$ operation with the medium and high $\mathrm{CN}$ high reactivity fuels produced substantially lower $\mathrm{NO}_{\mathrm{x}}$ emissions with a minimal increase in soot emissions. At the high load operating condition, a correlation of lower $\mathrm{NO}_{\mathrm{x}}$ emissions with medium and high $\mathrm{CN}$ fuels with lower T90 was observed indicating that the enhanced vaporization offered by low $\mathrm{T} 90$ fuels benefitted $\mathrm{RCCl}$ operation and $\mathrm{NO}_{\mathrm{x}}$ emissions as hypothesized. Also stated in hypothesis 1 , combustion with the low AC, high CN fuels did not result in as high of soot measurements as FACE 8 (high AC, high CN). However, with regards to CO and THC emissions, the hypothesis was not validated. FACE 5 (high CN, low AC, and low T90) did not provide lower $\mathrm{CO}$ and THC emissions than the other high $\mathrm{CN}$ fuels, although it also used a greater percentage CNG which ultimately had a dominant influence THC emissions and potentially CO emissions. Hypothesis 2, which postulated that a low CN fuel with a high AC would provide lower CO and THC 
emissions was also disproven. FACE 1, the only low CN, low AC fuel provided lower CO and THC emissions when compared to the other low CN fuels.

\subsection{Efficiency}

Efficiency of an ICE can be characterized by several different metrics. Combustion efficiency quantifies the unburned fuel or incomplete combustion products as a fraction of the total fuel energy input to the engine. As defined by Equation 33, the unburned fuel and incomplete combustion products were measured or calculated from the exhaust emissions and included $\mathrm{CO}, \mathrm{H}_{2}, \mathrm{THC}$, and soot or particulates. Another efficiency quantification, BTE, evaluates the work achieved by combustion with respect to the fuel energy input that was combusted or in other words, the heat released by the fuel that was converted to work output by the crankshaft. To quantify the fuel energy that was combusted, the combustion efficiency is multiplied by the mass flow of fuel and its energy content as described by Equation 35. The concern with presenting BTE is that when combustion efficiency is poor, the perception of efficiency can be inflated because of the combustion efficiency term in the denominator of the BTE equation. Furthermore, $\mathrm{RCCl}$ combustion, like other advanced combustion strategies, exhibits lower combustion efficiency than CDC. Thus for comparison sake it is more appropriate to present the FCE which is identical to BTE, except combustion efficiency is eliminated from the equation.

\subsubsection{Combustion Efficiency}

Combustion efficiency, by definition, is predominantly a function of the $\mathrm{CO}$ and THC emitted through the engines exhaust. Consequently, combustion efficiency followed the high reactivity fuel property trends set forth by $\mathrm{CO}$ and THC emissions. This is apparent in Figure 60, where the low CN fuels exhibited lower combustion efficiency related to their substantial THC emissions.

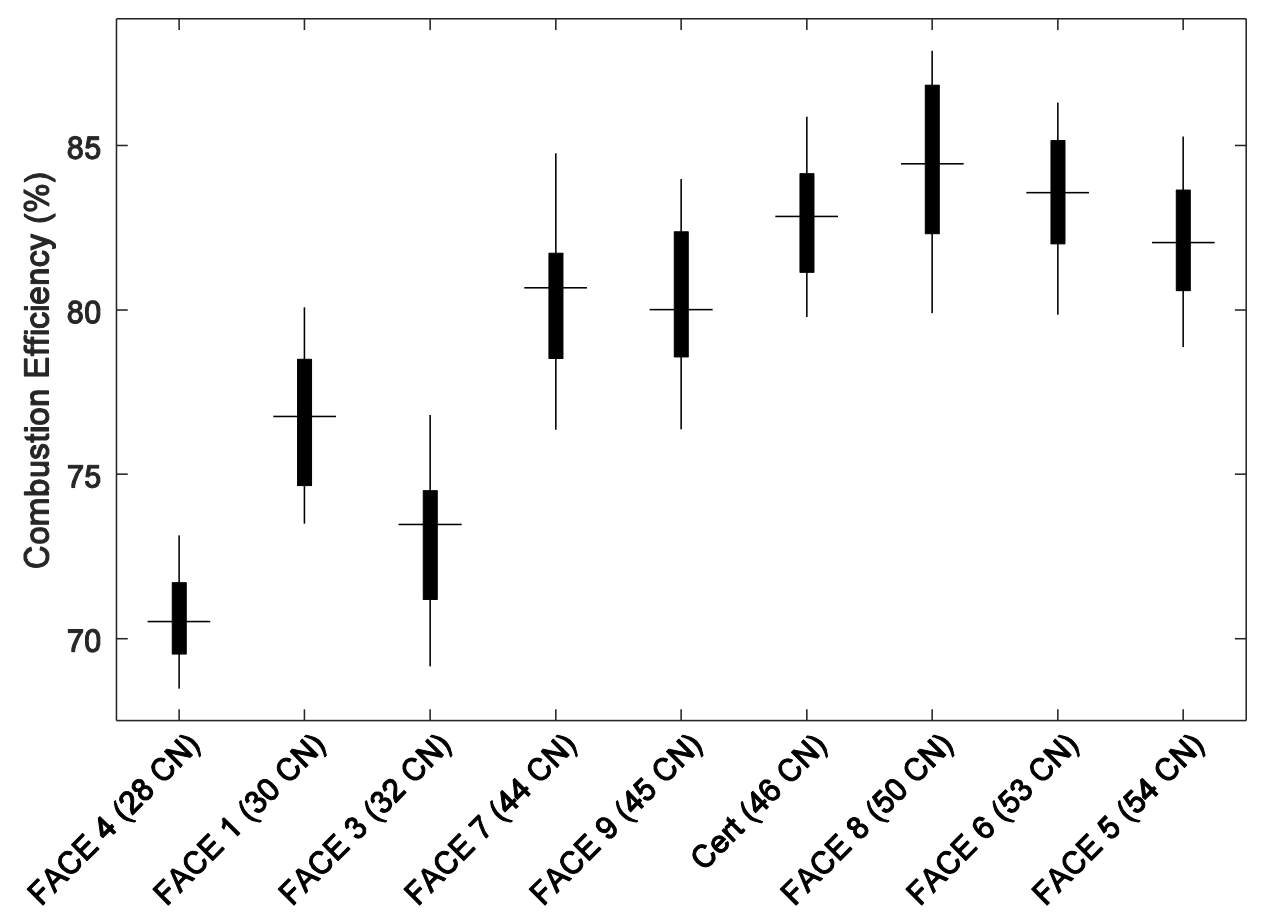

Figure 60: Combustion Efficiency at the Low Load Operating Condition (Varying IMAP and CA50) 
Analogous to the effects of $\mathrm{CN}$ of the high reactivity fuel on THC and $\mathrm{CO}$ emissions, combustion efficiency trends positively with increasing $\mathrm{CN}$. The effects of percentage $\mathrm{CNG}$ and fuel properties that were influential on THC and $\mathrm{CO}$ emissions can also be observed in the combustion efficiency data plotted in Figure 60. For example, note that FACE 6 and FACE 5 demonstrate a lower combustion efficiency than FACE 8, which corresponds to their higher, on average, THC emissions which was attributed to the higher percentage CNG used for these fuels to maintain combustion phasing. Conversely, $\mathrm{RCCl}$ operation with FACE 1 produced, on average, lower $\mathrm{CO}$ and THC emissions than the other low CN fuels (FACE 4 and FACE 3), and demonstrated substantially higher overall combustion efficiency. A least squares fit model developed with JMP further confirmed these associations as evidenced simply by the terms in Equation 53.

\section{Equation 53: Least Squares Fit Model for Combustion Efficiency at the Low Load Operating Condition}

\section{Combustion Efficiency}

$$
\begin{aligned}
& =115.56+0.76793 * C N-0.97629 * \text { Percentage } C N G+7.2502 * 10^{-3} \\
& *(C N-43.003) *(T 10-198.14)-0.031835 * A C
\end{aligned}
$$

By no coincidence, all of the terms in Equation 53 have been discussed previously as influences to THC or $\mathrm{CO}$ emissions. Furthermore, the least squares fit model possessed an $\mathrm{R}^{2}$ value of 0.91 and the $\mathrm{p}$ values for all parameters were less than 0.0001 . The model indicated that $\mathrm{CN}$ and percentage $\mathrm{CNG}$ were the most influential parameters. The term with $\mathrm{T} 10$ also included $\mathrm{CN}$ and thus the model suggested that the slope of the effect of T10 was dependent on $\mathrm{CN}$, which was demonstrated in Figure 57 for THC emissions. Note that under a CN of 43 (low CN fuels) the effect of T10 on THC emissions, and subsequently combustion efficiency was reversed.

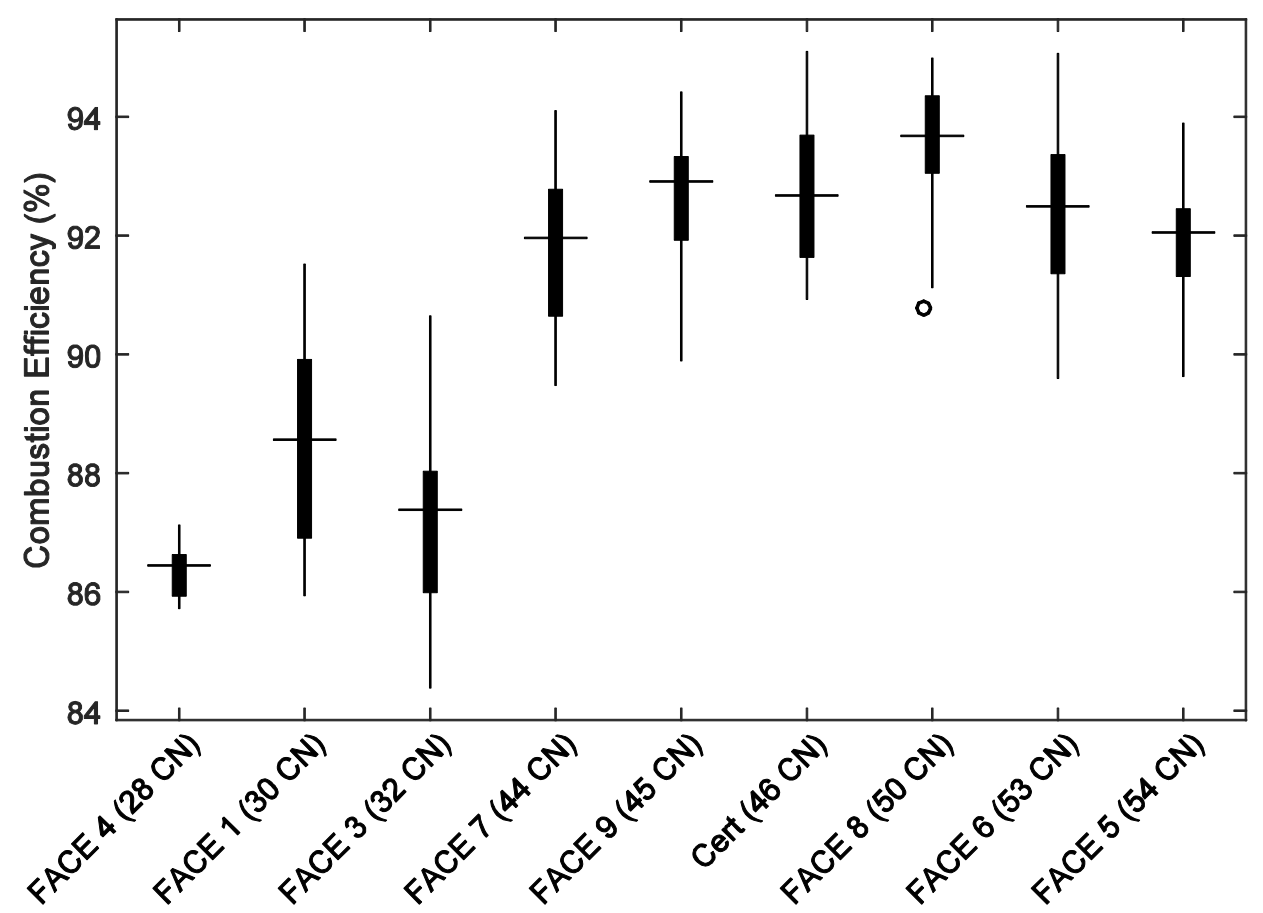

Figure 61: Combustion Efficiency at the High Load Operating Condition (Varying Main DI SOI Timing and CA50) 
At the high load operating condition, trends of fuel property effects and percentage CNG on combustion efficiency were nearly identical to the low load operating condition. Investigating the trends with a least squares fit model resulted in an equation with terms similar to the low load operating condition with the exception of no CN multiplied by T10 term. This term was excluded because it did not significantly increase the $R^{2}$ and did not have a $p$ value less than 0.0001 . Furthermore, the coefficients in Equation $54\left(R^{2}\right.$ of 0.89 ), which describes the least squares fit model for combustion efficiency at the high load operating condition, all have the same sign and are similar in order of magnitude to those in Equation 53.

\section{Equation 54: Least Squares Fit Model for Combustion Efficiency at the High Load Operating Condition}

Combustion Efficiency $=115.84+0.33871 * C N-0.52027 *$ Percentage CNG $-0.040929 * A C$

\subsubsection{Fuel Conversion Efficiency}

As noted previously, FCE, rather than BTE was considered a more appropriate method to quantify fuel property effects on the fuel efficiency of the engine for this study. As defined by Equation 35, BTE represents the efficiency of the engine in converting the heat released by the fuel to work output by the crankshaft. FCE, defined by Equation 36, represents the efficiency of the engine in converting fuel energy to work output by the crankshaft. When combustion efficiency is low, as can be the case with $\mathrm{RCCl}$ compared to $\mathrm{CDC}$, the BTE can be high while the FCE is actually low. For a commercial application, the consumer would not be interested in a high BTE if in fact the FCE was low compared to other technologies. Figure 62 demonstrates the misconception that could be provided by using BTE as a metric for fuel efficiency.

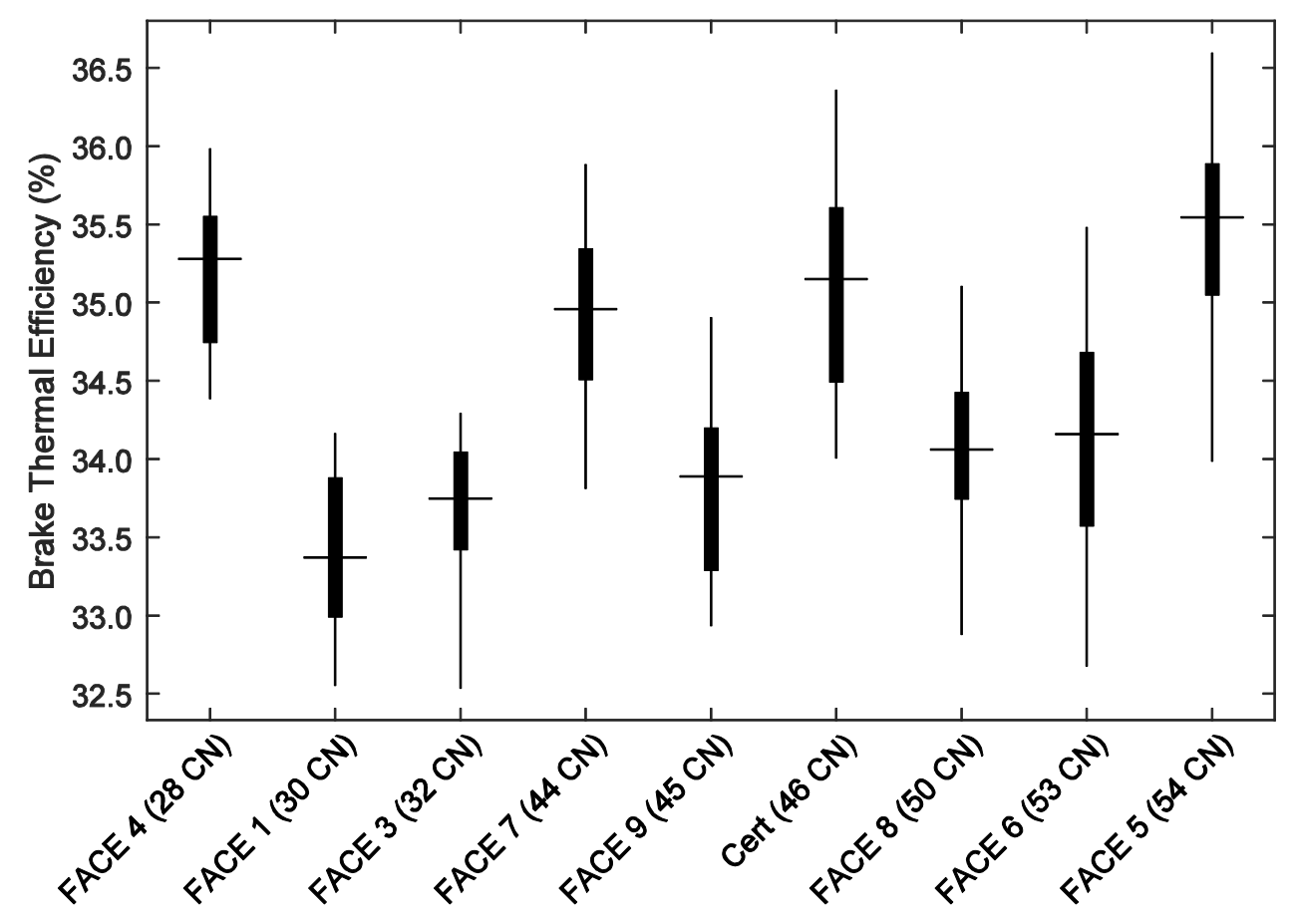

Figure 62: BTE at the Low Load Operating Condition (Varying IMAP and CA50) 
Comparing the BTE presented in Figure 62 to the FCE in Figure 63, it becomes apparent that using BTE to quantify fuel efficiency could result in contradictory conclusions. Notably, the BTE for FACE 4 was in the upper echelon of BTE measurements, yet the FCE was in the lower echelon when compared amongst all the high reactivity fuels.

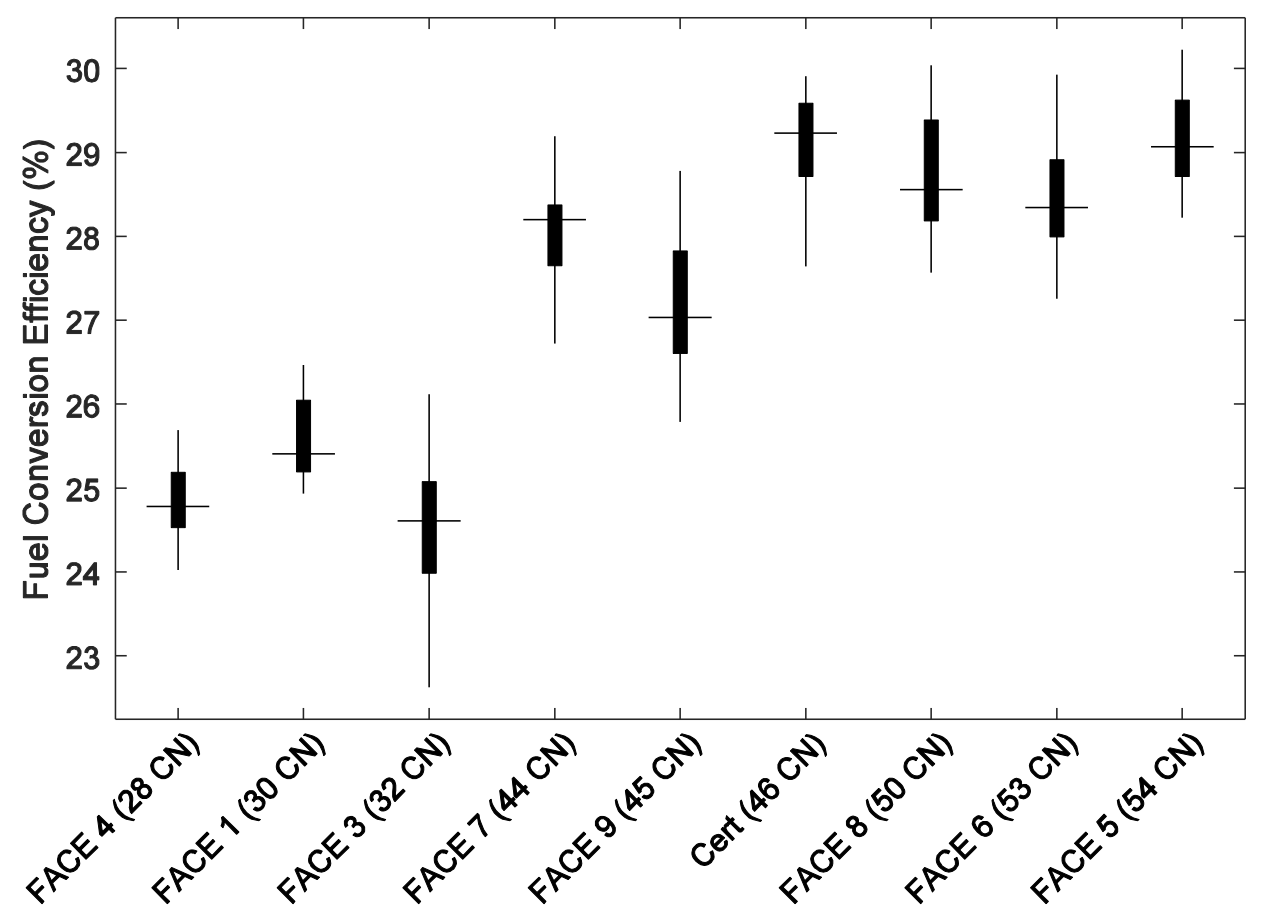

Figure 63: FCE at the Low Load Operating Condition (Varying IMAP and CA50)

Figure 63 demonstrates that at the low load operating condition, $\mathrm{CN}$ of the high reactivity fuel influenced the FCE. However, the influence was not absolute; there appears to be a CN threshold in which FCE increased to and then remained relatively comparable for higher $\mathrm{CN}$ fuels. The variations in FCE within the CN groupings suggested that there were other fuel properties that influenced FCE. Further investigation with a least squares fit performed with JMP suggested that percentage CNG, DI SOI timing, and AC were also influential on FCE. The model developed by JMP, detailed by Equation 55, exhibited an $R^{2}$ value of 0.93 and all included parameters had $p$-values less than 0.0001 .

\section{Equation 55: Least Squares Fit Model for FCE at the Low Load Operating Condition}

$$
\begin{aligned}
F C E=40.619 & +0.34177 * C N-0.28051 * \text { Percentage } C N G+4.2119 * 10^{-3} *(A C-32.762)^{2} \\
& +5.9176 * 10^{-3} *(C N-43.003) *(A C-32.762)-0.18230 * \text { DI SOI }
\end{aligned}
$$

The influences of AC are visible in Figure 63. Note that FACE 1, Cert, and FACE 5 are all low AC fuels and achieved higher FCE than other high reactivity fuels with similar CN. However, FACE 6 is also a low AC fuel, yet its FCE was similar to FACE 8, another high CN fuel, which has a high AC. The fact that FCE was not distinctly higher for FACE 6 versus FACE 8 is conceivably explained by the higher percentage CNG that was necessary to maintain proper combustion phasing for RCCl operation with FACE 6 as shown in Figure 24. Additionally, note that a negative coefficient exists for the percentage CNG term in Equation 55 , indicating that higher percentage CNG reduces FCE at the low load operating condition. This was 
related to the reduced combustion efficiency provided by high percentage CNG because of the propensity of the low reactivity fuel to reach crevices and other areas of the combustion chamber where the flame does not propagate resulting in higher levels of unburned fuel.

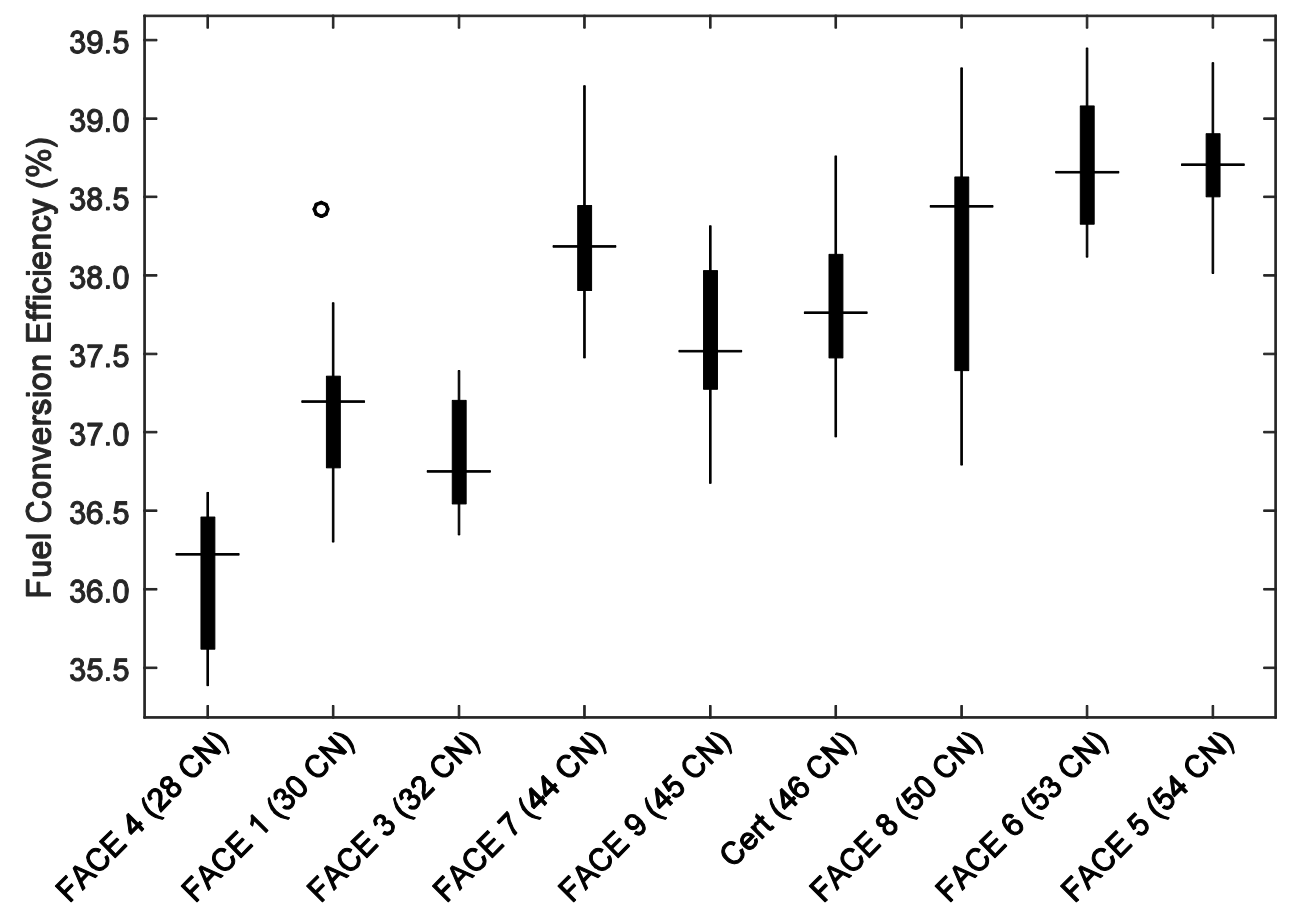

Figure 64: FCE at the High Load Operating Condition (Varying Main DI SOI Timing and CA50)

Similar fuel property trends were observed for FCE at the high load operating condition displayed Figure 64. The medium and high CN fuels achieved greater FCE than the low CN fuels. A least squares fit analysis with JMP suggested that percentage CNG, main DI SOI timing, CA50 and AC were influential on FCE in addition to $\mathrm{CN}$ at the high load operating condition. However, the $\mathrm{CN}$ and $\mathrm{AC}$ terms are found to be dependent on each other and do not appear alone. Equation 56 defines the FCE model at the high load operating condition, which exhibited an $\mathrm{R}^{2}$ of 0.76 .

Equation 56: Least Squares Fit Model for FCE at the High Load Operating Condition

$$
\begin{gathered}
F C E=23.471+0.16623 * \text { Percentage } C N G+0.049551 * \text { main DI SOI }+2.0163 \\
* 10^{-3}(C N-43.596) *(A C-32.483)-0.16327 * C A 50
\end{gathered}
$$

The absence of individual $A C$ and $C N$ terms was potentially due to the trends found within the medium $\mathrm{CN}$ fuels. The low AC fuels in the low and high $\mathrm{CN}$ fuel groupings all exhibited higher FCE on average than their counterparts, but for the medium CN fuels, the highest AC fuel, FACE 7, achieved the highest FCE on average.

\subsubsection{Efficiency Summary}

It can be concluded that combustion efficiency was not only a function of $\mathrm{CN}$, but also percentage CNG and $A C$. The influence of percentage CNG has been related to the flame front being extinguished for the low reactivity fuel. Additionally, Section 6.1 described how AC influenced percentage CNG and provides 
an explanation for its influence on combustion efficiency. Furthermore, the influence of $A C$ and percentage CNG has been directly linked to $\mathrm{CO}$ and THC emissions, which contribute significantly to combustion efficiency. CN, AC and percentage CNG were also present in the least squares fit models for FCE. This was due to the fact that un-combusted fuel, which is quantified by combustion efficiency, does not contribute to brake power output and thus positively to FCE.

\subsection{Operable Load Range}

A predominant obstacle of commercially implementing advanced combustion strategies is a limited operating range compared to conventional combustion strategies. Compared to other advanced combustion strategies that use a single fuel, $\mathrm{RCCl}$ does offer an additional level of flexibility to control combustion phasing through the adjustment of the fuel reactivity based on the ratio of the high reactivity fuel to the low reactivity fuel. Nonetheless, $\mathrm{RCCl}$ combustion was limited at high loads by excessive in-cylinder PRRs and at low loads by combustion stability, low combustion efficiency, and misfire concerns as observed during this research.

As noted in Section 5.3, the DI SOI timing had to be retarded for the low CN fuels when operating at both load conditions. Attempting to achieve $\mathrm{RCCl}$ combustion with the low $\mathrm{CN}$ fuels at the $\mathrm{DI}$ SOI timing used for medium and high $\mathrm{CN}$ fuels would result in a misfire regardless of the settings used for other engine control parameters. The misfire was identified in real time by an in-cylinder pressure curve that resembled a motoring curve, viewed on the combustion analysis DAQ computer. The following cycle would then exhibit a PRR in excess of $10 \mathrm{bar} / C A^{\circ}$ due to the residual fuel left in the cylinder by the misfire. To safely test the low CN fuels at the low load operating condition the DI SOI timing for FACE 3 and FACE 1 was retarded to $40^{\circ}$ BTDC. FACE 4, which had the lowest $C N$ number of all the fuels tested, as well as a high AC and T90, required a DI SOI timing of $35^{\circ}$ BTDC. It was hypothesized that the longer ignition delay exhibited by these low $\mathrm{CN}$ fuels resulted in a mixture with the natural gas and air that was not stratified enough for consistent combustion. Although advancing the DI SOI timing allowed for operation with the low $\mathrm{CN}$ fuels without consistent misfire, exhaust emissions of $\mathrm{NO}_{\mathrm{x}}$ increased by an order of magnitude in some instances (Figure 47 provides an example) compared to the medium and high CN fuels. Furthermore, in-cylinder PRRs for the low CN fuels were a concern even at the low load condition. This was especially prevalent for FACE 4 for which certain points on the test matrix had to be skipped due to PRRs over 10 bar $/ \mathrm{CA}^{\circ}$. A trend of decreasing in-cylinder PRR as $\mathrm{CN}$ increases can be observed in Figure 29. Hypothesis 2 stated that a high $A C$ in a low $C N$ high reactivity fuel could reduce combustion instabilities, which was found to be accurate based on the fact that FACE 4 and FACE 3, both high AC, low CN fuels, exhibited slightly lower COV of IMEP, on average, at the low load operating condition compared to FACE 1 (low CN, low AC). However, the COV of IMEP was not the limiting factor on operation at this load condition, in-cylinder PRR was, for which FACE 1 demonstrated, on average, lower PRR than FACE 4 and FACE 3, and therefore was better suited for the low load operation.

The medium and high CN fuels were not limited at the low load operating condition by PRR or misfire like the low CN fuels. The COV of IMEP approached and exceeded 10 percent in certain instances for the high CN fuels at the low load operating condition. However, the high COV of IMEP corresponded to high percentage CNG rates necessary to retard combustion phasing to achieve the highest CA50 targets. It is surmised that the medium and high $\mathrm{CN}$ fuels could operate at lower loads than the 3.6 bar BMEP tested. Furthermore, it is possible that the high CN fuels could have benefitted from a further advanced DI SOI timing which could have potentially reduced the percentage CNG necessary for combustion phasing. 
In-cylinder PRR was the sole limiting factor in increasing the maximum achievable load during RCCI combustion. As noted previously, $\mathrm{RCCl}$ combustion with the low $\mathrm{CN}$ high reactivity fuels produced incylinder PRR near and even exceeding $10 \mathrm{bar} / \mathrm{CA}^{\circ}$. A trend of decreasing in-cylinder PRR with increasing $\mathrm{CN}$ of the high reactivity fuel for the high load operating condition can be observed in Figure 31 . This suggested that the engine load could be further increased from the 6 bar BMEP set point used during the high load tests for the high $\mathrm{CN}$ high reactivity fuels. Subsequently, it can be concluded that utilizing high $\mathrm{CN}$ high reactivity fuels allows for the largest load range during $\mathrm{RCCl}$ operation. 


\section{Conclusions and Recommendations}

How do high reactivity fuel properties, such as $\mathrm{CN}, \mathrm{AC}$, and T90 affect diesel-natural gas RCCI combustion characteristics, fuel efficiency, exhaust emissions, and the operable load range? This fundamental question led to the development of two hypotheses regarding the impact of high reactivity fuel properties on $\mathrm{RCCl}$ combustion. To investigate these hypotheses, an applied research experiment was conceived. A GM 1.9 Liter, light-duty CIDI engine was retrofitted with gaseous injectors in the intake manifold and control system changes were made to facilitate RCCl combustion. The high reactivity fuels consisted of diesel fuels with varying properties, known as the FACE diesel fuels [33], and a certification grade diesel fuel. These fuels were injected directly into the cylinder using the factory injectors and a third-party injector controller. The low reactivity fuel, CNG, was injected into the intake manifold runner. The engine was operated at a single speed, 2100 RPM, and two different loads, 3.6 bar BMEP and 6 bar BMEP. Preliminary experiments were conducted to determine which engine control parameters had the largest effect on $\mathrm{RCCl}$ exhaust emissions, combustion characteristics, and fuel efficiency. The results from these studies were used to create test matrices for each load condition. Data was subsequently collected at each point of the test matrix for all of the high reactivity fuels, with the exception of test points that resulted in detrimentally high in-cylinder PRRs above $10 \mathrm{bar} / \mathrm{CA}^{\circ}$. These data were analyzed for fuel property influences and trends with the aid of screening analyses and least squares fit models developed by JMP statistical software [47].

\subsection{Conclusions}

$\mathrm{RCCl}$ combustion relies on a reactivity difference between two fuels to achieve a final fuel mixture reactivity that is required for proper combustion phasing. The required reactivity of the fuel mixture, or ratio of the low-to-high reactivity fuel, is dependent on engine speed and load. CN is the primary means of defining this reactivity and its effect on $\mathrm{RCCl}$ combustion, exhaust emissions, and fuel efficiency was overarching as demonstrated by the results presented herein. As a result, the high reactivity fuels were grouped into three categories based on $\mathrm{CN}$ : low ( $<44 \mathrm{CN})$, medium $(44>\mathrm{CN}<50)$, and high ( $>50 \mathrm{CN})$. Within these categories, the effects of other fuel properties were investigated and identified as influential, particularly AC and T10. T90 has previously been used as a measure of back end volatility and it is one of the properties that the FACE diesel fuels matrix was developed around [33]. However, statistical analysis performed with JMP software on data obtained from this research found that T10 was more influential in most instances compared to T90. Knowledge of these fuel property effects was then used to prove or disprove the two hypotheses that were stated in Chapter 3 and restated below.

Hypothesis $1-R C C l$ with a high $C N$ fuel with a low AC and a low T90 will reduce combustion instabilities and $\mathrm{THC}$ and $\mathrm{CO}$ emissions (compared to other high reactivity fuels) at low load by reducing ignition delay (CN) and promoting vaporization (low T90) while retaining low soot and NOx emissions (AC). Heavy dilution through EGR and multiple diesel injections per cycle will assist in reducing PRR during combustion of this fuel at high loads.

At the low load operating condition, it was observed that the COV of IMEP, a measure of combustion stability, was highest for the high $\mathrm{CN}$ high reactivity fuels, which does not support hypothesis 1 . On average, the high CN fuels operated at a COV of IMEP of 5.61 percent, the medium CN fuels operated at 3.22 percent, and the low CN fuels operated at 3.00 percent. However, DI SOI timing for the low CN fuels had to be retarded to avoid misfire which otherwise would have certainly resulted in a very high COV of IMEP if left unchanged from the medium and high CN DI SOI timing. Furthermore, an 
exponential correlation between COV of IMEP and percentage CNG was identified. High percentage CNG, which was necessary to achieve the most retarded combustion phasing targets (measured by CA50) for the high CN fuels resulted in a higher COV of IMEP. In fact, when the CA50 targets were closer to TDC, COV of IMEP measurements for the high CN fuels were comparable to the medium and low CN fuels and a substantial increase in $\mathrm{NO}_{x}$ emissions or in-cylinder PRR was not observed. At the low load operating condition, the $\mathrm{NO}_{x}$ emissions measurements for the medium and high $\mathrm{CN}$ fuels were similar and resulted in an average $\mathrm{BSNO}_{\mathrm{x}}$ of $0.169 \mathrm{~g} / \mathrm{bhp}$-hr while the low $\mathrm{CN}$ fuels produced an average of 1.58 g/bhp-hr. No discernible trends besides CN were observed at the low load condition; however, at the high load condition a T90 trend was observed amongst the medium and high CN fuels. As T90 of the high reactivity fuel decreased, $\mathrm{NO}_{x}$ emissions also decreased. For example, FACE 5 a low T90, high CN fuel produced 56.2 percent lower BSNOx emissions, on average, than FACE 8 , the high CN fuel with the highest T90. Similarly, for the medium CN fuels, FACE 7, a low T90 fuel, produced 93.6 percent lower $\mathrm{BSNO}_{\mathrm{x}}$ emissions, on average, compared to the highest T90 medium CN fuel, FACE 9. AC of the high reactivity fuel had no discernible effect on $\mathrm{NO}_{\mathrm{x}}$ emissions contrary to hypothesis 1 .

With regards to $\mathrm{CO}$ and THC emissions, hypothesis 1 was not validated. FACE 5, the high CN fuel with a low AC and T90 (as well as T10) did not provide lower CO and THC emissions compared to the other high CN fuels. In fact, amongst all of the high CN fuels, THC emissions measurements for FACE 5 were the highest, $29.4 \mathrm{~g} / \mathrm{bhp}-\mathrm{hr}$ and $10.4 \mathrm{~g} / \mathrm{bhp}$-hr for the low and high load operating conditions, respectively, which can be compared to $28.1 \mathrm{~g} / \mathrm{bhp}-\mathrm{hr}$ and $9.9 \mathrm{~g} / \mathrm{bhp}$-hr for FACE 6, and $25.3 \mathrm{~g} / \mathrm{bhp}-\mathrm{hr}$ and $8.4 \mathrm{~g} / \mathrm{bhp}$ $\mathrm{hr}$ for FACE 8. The higher THC emissions for FACE 5 and FACE 6 were attributed to the fact that they used a greater percentage CNG than FACE 8.

At the high load operating condition, the high $\mathrm{CN}$ fuels produced the lowest, on average, in-cylinder PRRs amongst the $\mathrm{CN}$ groupings. The high $\mathrm{CN}$ fuels averaged an in-cylinder PRR of $7.25 \mathrm{bar} / \mathrm{CA}^{\circ}$, while the medium and low $\mathrm{CN}$ fuels averaged $7.93 \mathrm{bar} / \mathrm{CA}^{\circ}$ and $8.77 \mathrm{bar} / \mathrm{CA}^{\circ}$, respectively. Introducing EGR to extend the maximum achievable load as suggested by hypothesis 1 had the opposite effect due to an increase in the IMAT. This increase in IMAT caused combustion phasing to advance and subsequently increased in-cylinder PRR. However, multiple diesel injections were employed in the form of a split DI that proved to quell in-cylinder PRR and $\mathrm{NO}_{x}$ emissions compared to a single injection event. The first injection contained 10 percent of the total quantity of DI fuel and began $1.3 \mathrm{~ms}$ prior to the primary $\mathrm{DI}$ SOI.

Hypothesis 2 - RCCl with a low CN fuel with high AC will extend high load operation (because of increased mixing time due to low CN), and reduce combustion instabilities as well as THC and CO emissions (compared to low CN fuels with a low AC) at low load operation based on the convention that fuels with higher AC exhibit higher combustion temperatures.

Potentially detrimental in-cylinder PRRs were an issue that plagued the low $\mathrm{CN}$ high reactivity fuels throughout the study. At both low and high load operating conditions, certain points on the test matrix had to be abandoned for FACE 4, the lowest CN high reactivity fuel, because of in-cylinder PRRs over 10 bar/CA ${ }^{\circ}$. This disproves the hypothesis that the low $\mathrm{CN}$ fuels would extend high load operation. In fact, the high $\mathrm{CN}$ fuels demonstrated the greatest potential to extend high load operation because they exhibited the lowest, on average in-cylinder PRR. The low CN high reactivity fuels with high AC, FACE 3 and FACE 4, did achieve lower COV of IMEP, on average, compared to FACE 1, a low CN fuel with a low AC, which supports hypothesis 2 . However, FACE 3 and FACE 4 were much more susceptible to high in- 
cylinder PRRs that proved to be more of a limiting factor to RCCI combustion than COV of IMEP. RCCI combustion with FACE 4 and FACE 3 resulted in average in-cylinder PRRs of $7.40 \mathrm{bar} / \mathrm{CA}^{\circ}$ and 7.51 bar $/ \mathrm{CA}^{\circ}$, respectively, while operating with FACE 1 resulted in an average of $6.68 \mathrm{bar} / \mathrm{CA}{ }^{\circ}$. FACE 1 also produced the lowest THC and $\mathrm{CO}$ emissions, which disproved the statement in hypothesis 2 of lower $\mathrm{THC}$ and $\mathrm{CO}$ emissions for RCCl operation with high AC, low $\mathrm{CN}$ fuels.

After addressing the hypotheses, it becomes apparent that the high $\mathrm{CN}$, high reactivity fuels were best suited for RCCl combustion amongst all the high reactivity fuels considered. Although the high $\mathrm{CN}$ fuels exhibited a higher average COV of IMEP at the low load condition compared to the medium and low CN fuels, they were able to operate at reasonable values. At the high load operating condition, the COV of IMEP was lower overall for medium and high CN fuels compared to low CN fuels. Furthermore, based on a trend of decreasing in-cylinder PRR as $\mathrm{CN}$ of the high reactivity fuel increased, the high $\mathrm{CN}$ fuels offered the widest operable load range. $\mathrm{NO}_{x}$ emissions from $\mathrm{RCCl}$ operation with the medium and high $\mathrm{CN}$ fuels were substantially lower than the low $\mathrm{CN}$ fuels. At the low load operating condition, $\mathrm{NO}_{\mathrm{x}}$ emissions were very similar amongst the medium and high $\mathrm{CN}$ fuels. A trend of decreasing $\mathrm{NO}_{\mathrm{x}}$ emissions as the T90 of the high reactivity fuel decreased was observed at the high load operating condition. A slightly higher soot emissions measurement was observed for the medium and high CN fuels, although it is important to note that only one test of all the fuels resulted in a brake specific soot measurement over $10 \mathrm{mg} / \mathrm{bhp}$-hr. Additionally, soot emissions were found to increase for high reactivity fuels with a higher T10.

From a utilization of natural gas standpoint, high CN and low AC fuels allowed for the highest percentage CNG among all the high reactivity fuels, which is desirable with respect to using a domestically abundant natural resource. This elevated percentage CNG did contribute to higher THC emissions, and subsequently lower combustion efficiency for comparisons made within the high $\mathrm{CN}$ grouping. However, FCE was not substantially affected and FACE 5, the high CN and low AC high reactivity fuel produced among the highest FCE of all the fuels tested. From these observations, it can be concluded that a high reactivity fuel with a high $\mathrm{CN}$, low AC, and low T10 allows for the widest operating range, largest percentage of low reactivity fuel, and high FCE while retaining low $\mathrm{NO}_{\mathrm{x}}$ and soot emissions.

\subsection{Recommendations}

The conclusions formed from this study were obtained from an engine operating at a single speed and two different loads. Although high reactivity fuel property effects on $\mathrm{RCCl}$ combustion were identified, these effects should also be substantiated at different engine speeds and loads as well as for other engines. For this research, time and financial constraints prevented further exploration. It can be stated with a high level of confidence that the medium and high $\mathrm{CN}$ high reactivity fuels were better suited for $\mathrm{RCCl}$ combustion. As such, examining the fuel properties, such as AC and T10 within this range of CN with a matrix of fuels that covers more combinations could provide further confidence in the conclusions. Based on the conclusion that the high $\mathrm{CN}$ fuels were best suited for $\mathrm{RCCl}$, experiments with higher $\mathrm{CN}$ fuels (greater than 54) are recommended to determine if the operable load range and fuel efficiency of $\mathrm{RCCl}$ combustion could be increased further.

Comparing $\mathrm{RCCl}$ operation with the low $\mathrm{CN}$ high reactivity fuels versus the medium $\mathrm{CN}$ fuels revealed a threshold for which $\mathrm{NO}_{x}$ emissions were greatly reduced (Figure 47 and Figure 49) while combustion efficiency (Figure 60 and Figure 61) and FCE (Figure 63 and Figure 64) were noticeably improved. Based 
on the $\mathrm{CN}$ of the highest low $\mathrm{CN}$ fuel and the lowest medium $\mathrm{CN}$ fuel, this threshold must lie between 32 and $44 \mathrm{CN}$. RCCl experiments with high reactivity fuels that have $\mathrm{CNs}$ in this range to determine where the threshold lies is recommended to determine if the ASTM-D975015c minimum of $40 \mathrm{CN}$ is applicable to $\mathrm{RCCl}$ operation.

$\mathrm{RCCl}$ with diesel fuel and natural gas provided high FCE with low $\mathrm{NO}_{\mathrm{x}}$ and soot emissions compared to $\mathrm{CDC}$. However, the substantial increase in $\mathrm{CO}$ and $\mathrm{THC}$ emissions, especially unburned $\mathrm{CH}_{4}$, presents a challenge for engineers. Attempting to use an oxidation catalyst to remediate these emissions is difficult based on the fact that exhaust temperatures are typically lower for $\mathrm{RCCl}$ combustion. Also, $\mathrm{CH}_{4}$, the predominate component of natural gas, has a much higher catalyst light off temperature than higher order hydrocarbons. Thus, research in mitigating $\mathrm{CO}$ and $\mathrm{THC}$ emissions from $\mathrm{RCCl}$ combustion, as well as developing catalysts that can oxidize $\mathrm{CH}_{4}$ at lower light off temperatures, could greatly enhance the commercialization of this promising technology. 


\section{References}

[1] W. Pulkrabek, Engineering Fundamentals of the Internal Combustion Engine, Upper Saddle River: Pearson Prentice-Hall, 2004.

[2] U.S. Department of Energy, "Clean Cities Alternative Fuel Price Report," Washington D.C., 2016.

[3] Westport Power Inc., "First Generation Westport HPDI Technology," 2016. [Online]. Available: http://www.westport.com/is/core-technologies/combustion/hpdi. [Accessed 12 January 2016].

[4] J. Smallwood, "Invesitgation of the Dual-Fuel Conversion of a Direct Injection Diesel Engine," Thesis, West Virginina University, Morgantown, WV, 2013.

[5] J. Egusquiza, C. Braga and S. Braga, "Experimental Investigation of a Diesel Cycle Engine Operating on Natural Gas/Diesel Dual-Fuel Mode," SAE Technical Paper 2011-36-0351, 2011.

[6] Z. Jia and I. Denbratt, "Experimental Investigation of Natural Gas-Diesel Dual-Fuel RCCI in a HeavyDuty Engine," SAE Technical Paper 2015-01-0838, 2015.

[7] A. Dempsey, R. Walker and R. Reitz, "Effect of Piston Bowl Geometry on Dual Fuel Reactivity Controlled Compression Ignition (RCCl) in a Light-Duty Engine Operated with Gasoline/Diesel and Methanol/Diesel," SAE Technical Paper 2013-01-0264, 2013.

[8] S. Curran, R. Hanson, R. Wagner and R. Reitz, "Efficiency and Emissions Mapping of RCCl in a LightDuty Diesel Engine," SAE Technical Paper 2013-01-0289, 2013.

[9] P. Glaude, R. Fournet, R. Bounaceur and M. Moliere, "Gas Turbines and Biodiesel: a Clarification of the Relative NOX Indices of Fame, Gasoil and Natural Gas," in Proceedings of ASME Turbo Expo 2009: Power for Land, Sea and Air, Orlando, 2009.

[10] L. C. Wagemakers A., "Review on the Effects of Dual-Fuel Operation, Using Diesel and Gaseous Fuels, on Emissions and Performance," SAE Technical Paper 2012-01-0869, 2012.

[11] J. Heywood, Internal Combustion Engine Fundamentals, New York: McGraw-Hill, Inc., 1988.

[12] P. Stalhammar, L. Erlandsson and K. Willner, "Assessment of the Dual-Fuel Technology," AVL, 2011.

[13] G. Neely, S. Sasaki, Y. Huang, J. Leet and D. Steward, "New Diesel Emission Control Strategy to Meet US Tier 2 Emissions Regulations," SAE Techincal Paper 2005-01-1091, 2005.

[14] D. Carder, R. Ryskamp, J. Nuszkowski, H. Li, N. Clark, G. Thompson, M. Gautam and S. Wayne, "CRC Report No. AVFL-16 Fuels to Enable Light-Duty Advanced Combustion Regimes," Coordinating Research Council, Alpharetta, 2012. 
[15] Wisconsin Engine Research Consultants, "Reactivity Controlled Compression Ignition (RCCI)," [Online]. Available: http://www.w-erc.com/services/rcci/. [Accessed 3 April 2014].

[16] S. Kokjohn, R. Hanson, D. Splitter and R. Reitz, "Experiments and Modeling of Dual-Fuel HCCl and PCCI Combustion Using In-Cylinder Fuel Blending," SAE Technical Paper 2009-01-2647, 2009.

[17] P. Dunbeck and R. Reitz, "An Experimental Study of Dual Fueling with Gasoline Port Injection in a Single-Cylinder, Air-Cooled HSDI Diesel Generator," SAE Techincal Paper 2010-01-0869, 2010.

[18] S. Curran, V. Prikhodko, K. Cho, C. Sluder, J. Parks, R. Wagner, S. Kokjohn and R. Reitz, "In-Cylinder Fuel Blending of Gasoline/Diesel for Inproved Efficiency and Lowest Possible Emissions on a MultCylinder Light-Duty Diesel Engine," SAE Technical Paper 2010-01-2206, 2010.

[19] S. Joo, T. Alger, C. Chadwell, W. Ojeda, J. Zuehl and R. Gukelberger, "A High Efficiency, Dilute Gasoline Engine for the Heavy-Duty Market," SAE Technical Paper 2012-01-1979, 2012.

[20] J. Sun, J. Bittle and T. Jacobs, "Influencing Parameters of Brake Fuel Conversion Efficiency with Diesel / Gasoline Operation in a Medium-Duty Diesel Engine," SAE Technical Paper 2013-01-0273, 2013.

[21] D. Splitter, M. Wissink, S. Kokjohn and R. Reitz, "Effect of Compression Ratio and Piston Geometry on RCCI Load Limits and Efficiency," SAE Technical Paper 2012-01-0383, 2012.

[22] R. Hanson, S. Curran, R. Wagner, S. Kokjohn, D. Splitter and R. Reitz, "Piston Bowl Optimization for RCCI Combustion in a Light-Duty Multi-Cylinder Engine," SAE Technical Paper 2012-01-0380, 2012.

[23] S. Curran, V. Prikhodko, Z. Gao, J. Parks, R. Wagner, G. Singh and K. Howden, "High Efficiency Clean Combustion in Mult-Cylinder Light-Duty Engines," in 2013 DOE Hydrogen Program and Vehicle Technolgies Annual Merit Review, 2013.

[24] V. Prikhodko, S. Curran, J. Parks and R. Wagner, "Effectiveness of Diesel Oxidation Catalyst in Reducing HC and CO Emissions from Reactivity Controlled Compression Ignition," SAE Technical Paper 2013-01-0515, 2013.

[25] D. Nieman, A. Dempsey and R. Reitz, "Heavy-Duty RCCI Operation Using Natural Gas and Diesel," SAE Technical Paper 2012-01-0379, 2012.

[26] R. Hanson, S. Curran, R. Wagner and R. Reitz, "Effects of Biofuel Blends on RCCl Combustion in a Light Duty, Multi-Cylinder Diesel Engine," SAE Technical Paper 2013-01-1653, 2013.

[27] S. Curran, R. Hanson and R. Wagner, "Effect of E85 on RCCI Performance and Emissions on a MultiCylinder Light-Duty Diesel Engine," SAE Technical Paper 2012-01-0376, 2012.

[28] D. Splitter, M. Wissink, D. DelVescovo and R. Reitz, "RCCI Engine Operation Towards 60\% Thermal Efficiency," SAE Technical Paper 2013-01-0279, 2013. 
[29] V. Soloiu, A. Rivero-Castillo, M. Muinos, M. Duggan, S. Harp, W. Peavy, S. Wolter and B. Vlcek, "Simultaneous Reduction of $\mathrm{NO}_{x}$ and Soot in a Diesel Engine through RCCI Operation with PFI of nbutanol and DI of Cottonseed Biodiesel," SAE Technical Paper 2014-01-1322, 2014.

[30] R. Hanson, S. Kokjohn, D. Splitter and R. Reitz, "Fuel Effects on Reactivity Controlled Compression Ignition (RCCI) Combustion at Low Load," SAE Technical Paper 2011-01-0361, 2011.

[31] D. Splitter, R. Reitz and R. Hanson, "High Efficiency, Low Emissions RCCI Combustion by Use of a Fuel Additive," SAE Technical Paper 2010-01-2167, 2010.

[32] A. Ickes, T. Wallner, T. Zhang and W. De Ojeda, "Impact of Cetane Number on Combustion of a Gasoline-Diesel Dual-Fuel Heavy-Duty Multi-Cylinder Engine," SAE Technical Paper 2014-01-1309, 2014.

[33] M. Alnajjar, B. Cannella, H. Dettman, C. Fairbridge, J. Franz, T. Gallant, R. Gieleciak, D. Hager, C. Lay, S. Lewis, M. Ratcliff, S. Sluder, J. Storey, H. Yin and B. Zigler, "CRC Report No. FACE-1 Chemical and Physical Properties of the Fuels for Advanced Combustion Engines (FACE) Research Diesel Fuels," Coordinating Research Council, Inc., Alpharetta, 2010.

[34] V. Hosseini, W. Neill, H. Guo, C. Dumitrescu, W. Chippior, C. Fairbridge and K. Mitchell, "Effects of Cetane Number, Aromatic Content and 90\% Distillation Temperature on HCCl Combustion of Diesel Fuels," SAE Technical Paper 2010-01-2168, 2010.

[35] K. Cho, M. Han, S. Sluder, R. Wagner and G. Lilik, "Experimental Investigation of the Effects of Fuel Characteristics on High Efficiency Clean Combustion in a Light-Duty Diesel Engine," SAE Technical Paper 2009-01-2669, 2009.

[36] W. De Ojeda, T. Bulicz, X. Han, M. Zheng and F. Cornforth, "Impact of Fuel Properties on Diesel Low Temperature Combustion," SAE Technical Paper 2011-01-0329, 2011.

[37] A. Azetsu, Y. Sato and Y. Wakisaka, "Effects of Aromatic Components in Fuel on Flame Tempearture and Soot Formation in Intermittent Spray Combustion," SAE Technical Paper 2003-01-1913, 2003.

[38] Drivven, Inc., "Drivven Home Page," [Online]. Available: http://www.drivven.com/. [Accessed 24 March 2016].

[39] West Virginia University Center for Alternative Fuels, Engines, and Emissions, Scimitar Capture, Computer Software, Morgantown, 2015.

[40] M. Moran and H. Shapiro, Fundamentals of Engineering Thermodynamics, Hoboken: John Wiley \& Sons, Inc., 2004.

[41] J. Nuszkowski, "The Effects of Fuel Additives on Diesel Engine Emissions during Steady State and Transient Operation," Dissertation, West Virginia University, Morgantown, 2008.

[42] Meriam Process Technologies, User Manual Laminar Flow Elements, Cleveland, 2015. 
[43] Z. Morvay and D. Gvozdenac, Applied Industrial Energy and Environmental Management, Hoboken: Wiley-IEEE Press, 2008.

[44] United States Government Printing Office, "Code of Federal Regulations Title 40: Protection of Environment Part 1065 - Engine Testing Procedures," 2015.

[45] Rosemount Analytical Inc., "Model 755R Oxygen Analyzer Instruction Manual," Rosemount Analytical, Inc., La Habra, 1991.

[46] H. Yun, J. Kang, M. Chang and P. Najt, "Improvement on Cylinder-to-Cylinder Variation Using a Cylinder Balancing Control Strategy in Gasoline HCCI Engines," SAE Technical Paper 2010-01-0848, 2010.

[47] SAS Institute Inc., JMP, Computer Software, Vers. 12.1.0, Cary, 2015.

[48] R. J. Freund, W. J. Wilson and D. L. Mohr, Statistical Methods, Philadelphia: Elsevier Inc., 2010.

[49] National Institue of Standards and Technology, Engineering Statistics Handbook, Washington D.C.: United States Department of Commerce, 2013.

[50] W. Majewski and H. Jääskeläinen, "Biodiesel-Mono Alkyl Esters," 2009. [Online]. Available: https://www.dieselnet.com/tech/fuel_biodiesel.php. [Accessed 9 March 2016].

[51] W. Majewski and H. Jääskeläinen, "What is Diesel Fuel," 2013. [Online]. Available: https://www.dieselnet.com/tech/fuel_diesel.php. [Accessed 9 March 2016].

[52] ASTM International, "D975-15c Standard Specification for Diesel Fuel Oils," West Conshohocken, 2015. 